Aus dem Institut für Allgemeinmedizin

(Prof. Dr. med. Eva Hummers-Pradier)

im Zentrum Innere Medizin

der Medizinischen Fakultät der Universität Göttingen

\title{
Verzicht als beherrschende Krankheitserfahrung bei Diabetes Typ 2
}

Eine qualitative Auswertung von Interviews

\author{
INAUGURAL - DISSERTATION \\ zur Erlangung des Doktorgrades \\ der Medizinischen Fakultät \\ der Georg-August-Universität zu Göttingen \\ vorgelegt von \\ Maike Buchmann \\ aus \\ Bonn
}

Göttingen 2014 
De k a n:

Prof. Dr. rer. nat. Heyo Klaus Kroemer

I. Berichterstatter: Prof. Dr. disc. pol. Wolfgang Himmel

II. Berichterstatter/in: Prof. Dr. Christoph Herrmann-Lingen

III. Berichterstatter/in: PD Dr. Mark Schweda

Promotor-Vertreterin: Prof. Dr. Margarete Schön

Tag der mündlichen Prüfung: $\quad$ 26.10.2016 


\section{Inhaltsverzeichnis}

1. Einleitung 1

2. Stand der Forschung 2

2.1 Diabetes - eine „Epidemie“ 2

2.2 Pathophysiologie und Ätiologie 4

2.3 Therapieoptionen 7

2.4 Diabetes als psychosoziales Problem - Selbstmanagement als Antwort 10

2.5 Krankheitskonzepte - ein integrales Modell 12

2.6 Krankheitskonzepte im Spannungsverhältnis 16

2.7 Misserfolge und Missverständnisse: Zuschreibung von Verantwortlichkeit 23

2.8 Zusammenfassung und Beurteilung 27

3. Fragestellung und Ziel 30

4. Methode 31

4.1 Theoretische Grundlagen 31

4.1.1 Erforschung von Lebenswelten 31

4.1.2 Qualitative Sozialforschung 32

4.2 Grounded Theory 33

4.2.1 Kodieren 34

4.2.2 Vergleiche 34

4.2.3 Theoretisches Sampling und theoretische Sättigung 36

4.3 Kontext und Material 36

4.3.1 „Krankheitserfahrungen.de“ 36

$\begin{array}{lll}\text { 4.3.2 Charakterisierung des Materials } & 37\end{array}$

$\begin{array}{lll}\text { 4.3.3 Narrative Interviews } & 39\end{array}$

4.4 Praktische Herangehensweise 39

4.4.1 Datenauswahl 39

$\begin{array}{ll}\text { 4.4.2 Kodier-Verfahren } & 41\end{array}$

4.4.3 Ausbildung und Nutzung theoretischer Sensibilität 43

$\begin{array}{lll}4.4 .4 & \text { Atlas.ti } & 44\end{array}$

4.4.5 Memos 44

4.5 Datenschutz und Genehmigung durch die Ethik-Kommission 45

5. Ergebnisse 46

5.1 Vorbemerkungen zur Kernkategorie und Darstellung der Ergebnisse 46

5.2 Beschreibung der Stichprobe 47

5.3 Kernkategorie: Verzicht als beherrschende Erfahrung bei Diabetes Typ 2 
5.4 Subkategorie 1: Verzicht ist schmerzhaft 50

5.4.1 Auf ein genussvolles Leben verzichten müssen 50

5.4.2 Benachteiligt sein 52

5.4.3 Gegen das eigene Wohlbefinden kämpfen müssen 56

$\begin{array}{ll}5.4 .4 & \text { Allein gelassen werden } \\ \end{array}$

5.5 Subkategorie 2: Verzicht wird moralisch bewertet 60

5.5.1 Laborwerte und Körpermaße als „sichtbare“ moralische Prüfmarken 61

5.5.2 Sich von „den Maßlosen“ abgrenzen - (un)schuldig sein 67

5.5.3 Körpermaße normieren, akzeptieren, verändern 73

5.5.4 Sich Anerkennung „unsichtbarer“ Bemühungen wünschen 76

5.6 Subkategorie 3: Verzicht wird geübt 79

5.6.1 Kontrolle von außen: Unterstützung oder Übergriff? 80

5.6.2 Laborwerte und Gewicht (nicht) kontrollieren können 84

5.6.3 Regeln formulieren: "gesunde“ und „ungesunde“ Lebensmittel 87

5.6.5 Neue positive Erfahrungen machen 96

$\begin{array}{ll}\text { 6. Diskussion } & 100\end{array}$

$\begin{array}{lll}\text { 6.1 Stärken und Schwächen } & 100\end{array}$

6.1 Verzicht: zwischen Entbehrung und moralischer Läuterung 103

6.2 Verzicht bei Diabetes Typ 2 - eine fast ungerechte Forderung 104

6.3 Moralisierung von Lebensstil, Körpermaßen, Krankheit 107

6.4 Eigene Regeln - Last oder Befreiung? 116

6.6 Praktische Implikationen 122

$\begin{array}{ll}\text { 7. Zusammenfassung } & 126\end{array}$

\begin{tabular}{ll} 
8. & Literaturverzeichnis \\
\hline
\end{tabular}

$\begin{array}{ll}\text { 9. Anhang } & 135\end{array}$

9.1 Memo zur Kernkategorie Verzicht 135

\section{Tabellen- und Abbildungsverzeichnis}

Abbildung 1: Kodieren mit Atlas.ti 45

Tabelle 1: Stichprobe $\quad 49$ 


\section{Einleitung}

Die vorliegende Arbeit untersucht Krankheitserfahrungen mit Diabetes Typ 2. Ziel ist es, die Lebensperspektive, Probleme und Hoffnungen von Menschen mit dieser Erkrankung besser zu verstehen.

Diabetes zählt zu den vordringlichsten Gesundheitsproblemen unseres Jahrhunderts. Dabei fällt der Typ 2 besonders ins Gewicht. Der weltweite Anstieg der Prävalenz wird mit einem Lebensstil assoziiert, der von Bewegungsarmut und ungesunder Ernährung geprägt ist. Es liegt nahe, dass gerade bei Diabetes die Primärprävention ein großes gesellschaftliches Anliegen ist, da die Erkrankung als "vermeidbar" gilt (International Diabetes Federation 2013). Ein gesunder Lebensstil ist nach der Diagnose „Basistherapie" (Bundesärztekammer (BÄK), Kassenärztliche Bundesvereinigung (KBV), Arbeitsgemeinschaft der Wissenschaftlichen Medizinischen Fachgesellschaften (AWMF) 2013). Der Lebensstil ist allerdings ein privater Bereich, auf den von außen, z.B. durch medizinisches Personal, schwerlich Einfluss genommen werden kann - Selbstmanagement der Betroffenen ist das Schlüsselwort in der Diabetestherapie.

Angesichts der steigenden Prävalenz haben sich viele Forschungsgruppen mit Problemen des Selbstmanagements und möglichen Interventionen beschäftigt. Zumeist wird den Betroffenen die Verantwortung für die mangelnde Eindämmung der „Epidemie" übertragen; nur selten steht deren subjektive Sichtweise auf die Erkrankung im Mittelpunkt des wissenschaftlichen Interesses.

Es ist davon auszugehen, dass Diabetes Typ 2 von Menschen auf spezifische Weise erlebt wird, die sich von den Erfahrungen mit anderen chronischen Krankheiten unterscheidet, bei denen Selbstmanagement weniger Bedeutung zugemessen wird, wie z.B. Multiple Sklerose. Spezifische Muster der Krankheitserfahrung aufzudecken, ist Ziel dieser Arbeit. 


\section{Stand der Forschung}

\subsection{Diabetes - eine „Epidemie“}

Diabetes mellitus ist eine weltweit auftretende folgenschwere Stoffwechselerkrankung und wird als "globale Bürde“ verstanden (Nolan et al. 2011; International Diabetes Federation 2013). Schätzungen der International Diabetes Federation (IDF) zufolge ist ein Anstieg der Prävalenz von Diabetes (Typ 1 und Typ 2) der erwachsenen Weltbevölkerung auf knapp 8,8\% im Jahr 2035 zu erwarten (International Diabetes Federation 2013). In Deutschland ist Diabetes laut IDF (2013) mit einer für das Jahr 2013 geschätzten altersstandardisierten Prävalenz von 8,27\% der Bevölkerung zwischen 20 und 79 Jahren im globalen Vergleich (8,26\%) durchschnittlich stark verbreitet. In der „Studie zur Gesundheit Erwachsener in Deutschland“ (DEGS1) des Robert Koch-Instituts wird die Lebenszeitprävalenz des bekannten Diabetes in Deutschland mit 7,2\% der Bevölkerung zwischen 18 und 79 Jahren etwas niedriger geschätzt (Heidemann et al. 2013).

Maßgeblich ist Diabetes Typ 2, welcher weltweit ca. 90\% des Diabetes ausmacht (World Health Organisation 2013). Während Diabetes Typ 1 in der Regel durch einen absoluten Insulinmangel nach Zerstörung der insulinproduzierenden B-Zellen des Pankreas durch ein Autoimmungeschehen gekennzeichnet ist, handelt es sich bei Typ 2 um eine Insulinresistenz der Körperzellen (International Diabetes Federation 2013). Diabetes Typ 2 ist zu Beginn in der Regel asymptomatisch, so dass die Krankheit oft erst Jahre später diagnostiziert wird, wenn es zu Komplikationen gekommen ist. Klassische Symptome des Diabetes Typ 1, wie ein vermehrtes Durstgefühl oder eine erhöhte Urinausscheidung, können auftreten, zeigen sich aber meistens nur in abgeschwächter Form (World Health Organisation 2013). Als Vorstufe des Diabetes Typ 2 gilt eine verminderte Glukose-Toleranz (Impaired Glucose Tolerance, IGT), die sich in grenzwertig erhöhten Blutzuckerwerten äußert. Auch diese Symptomatik ist mit einer für 2013 geschätzten Prävalenz von 6,9\% der erwachsenen Bevölkerung weltweit stark verbreitet (International Diabetes Federation 2013).

Diabetes Typ 2 hat sich zu einer regelrechten „Epidemie“ entwickelt (Colagiuri et al. 2005; Rubin 2005; Nolan et al. 2011) die keineswegs - wie die im deutschen Sprach- 
raum verbreiteten Bezeichnungen "Wohlstandskrankheit“ und „Altersdiabetes" suggerieren - nur die ältere Bevölkerung der Industrienationen betrifft. In den von der von den UN als entwickelt bezeichneten Ländern wird sich die Zahl der Erwachsenen mit Diabetes zwischen 2010 und 2030 um 20\% erhöhen, in den sich in Entwicklung befindlichen Ländern um 69\% (Shaw et al. 2010). Die stark erhöhte Inzidenz wird auf die zunehmende Urbanisierung und Alterung der Gesellschaften und einen von Bewegungsmangel gekennzeichneten Lebensstil zurückgeführt (Shaw et al. 2010). Heute müssen vor allem die sogenannten Low \& middle income countries (LMC), in denen 80\% der Menschen mit Diabetes leben, die hohen Krankheitskosten stemmen (International Diabetes Federation 2013). Weltweit ist ein niedriger sozioökonomischer Status mit dem erhöhten Risiko, an Diabetes zu erkranken, assoziiert (Agardh et al. 2011). Dieser Zusammenhang scheint auch für Deutschland zu gelten (Heidemann et al. 2013). Zunehmend betrifft Diabetes Typ 2 auch jüngere Menschen (Colagiuri et al. 2005), mit dramatischen Auswirkungen auf die Lebenserwartung (Rhodes et al. 2012).

Diabetes geht mit einer hohen Komorbidität und Mortalität einher. Laut International Diabetes Federation (2013) sind 2013 weltweit ungefähr 5,1 Millionen Menschen zwischen 20 und 79 Jahren an Diabetesfolgen verstorben, was 8,4\% der Gesamtmortalität in dieser Altersgruppe entspricht. Die zahlreichen Komplikationen und Begleiterkrankungen sind auf eine chronische Schädigung der Nerven und Gefäße zurückzuführen. Retinopathien, Nephropathien, Neuropathien, Fußulzera, Hypertonie und kardiovaskuläre Erkrankungen entwickeln sich meist unbemerkt über einen längeren Zeitraum hinweg und können lebensbedrohlich verlaufen. Auch die nichtalkoholischen Fettleber und Leberentzündungen, das Polyzystische Ovarialsyndrom und möglicherweise einige maligne Erkrankungen sind mit Diabetes assoziiert (Nolan et al. 2011). Etwa die Hälfte der Erkrankten stirbt laut World Health Organisation (2013) an einer kardiovaskulären Folgeerkrankung, meist an einem Schlaganfall oder einem Herzinfarkt. Diabetes stellt die häufigste Ursache für Erblindung im Erwachsenenalter in den USA dar (Centers for Disease Control and Prevention 2011).

Heller et al. (2014) weisen allerdings darauf hin, dass in Lehrbüchern und Leitlinien Prävalenzen von Folgeerkrankungen überschätzt werden. Sie kritisieren z.B., dass die Häufigkeit einer Retinopathie bei Diabetes Typ 2 bei einer Krankheitsdauer von 10 Jahren einschlägigen Studien zufolge nur 10-15\% entspricht, während in den Leitlinien 
angegeben wird, dass zum Zeitpunkt der Diagnose schon jede dritte Person Komplikationen an der Netzhaut habe.

Infolge der schwerwiegenden Folgeerkrankungen und Behinderungen ergeben sich große ökonomische Belastungen. Direkte Kosten durch lange Krankenhausaufenthalte und Therapien wurden in den USA für das Jahr 2007 auf 116 Mrd. Dollar geschätzt, während sich indirekte Kosten wie Krankheitstage und Produktionsausfälle auf Kosten von zusätzlich $58 \mathrm{Mrd}$. Dollar beliefen (Centers for Disease Control and Prevention 2011). 2001 entfielen in Deutschland 30,6 Mio. Euro an direkten Ausgaben auf die Versorgung von Menschen mit Diabetes. Die Exzess-Kosten, d.h. die im Vergleich mit Nicht-Erkrankten zusätzlich entstandenen Kosten, beliefen sich auf 14,6 Mio. Euro, pro Person mit Diabetes sind das ca. 2.500 Euro mehr Versorgungskosten als pro Person ohne diese Erkrankung. Darüber hinaus werden durch Arbeitsunfähigkeit und Frühberentung zusätzlich erhebliche indirekte Kosten verursacht (Köster et al. 2006). Der Verbreitung und Auswirkungen von Diabetes Typ 2 effektiv entgegenzutreten, gilt als große Herausforderung der modernen Gesellschaft (Nolan et al. 2011).

\subsection{Pathophysiologie und Ätiologie}

Kennzeichen des Diabetes Typ 2 sind eine erworbene Insulinresistenz und Insulinsekretionsstörung (Matthaei et al. 2011). Insulin hält bei Gesunden zusammen mit anderen Peptidhormonen den normalen Blutzuckerspiegel aufrecht. Nimmt die Glukosekonzentration im Blutkreislauf des Körpers nach einer Mahlzeit zu, werden die B-Zellen des Pankreas beim Gesunden dazu angeregt, Insulin auszuschütten (Nolan et al. 2011). Insulin sorgt für eine Aufnahme der Glukose in insulinsensible Gewebe wie den Skelettmuskel, das Herz sowie das Fettgewebe und gewährleistet so eine normale Glukosekonzentration im Blut. Währenddessen wird die Freisetzung von Glukagon aus den $\alpha$-Zellen des Pankreas unterdrückt, sodass dieser "Gegenspieler" des Insulins nicht wie im nüchternen Zustand die endogene Glukoseproduktion, z.B. in der Leber stimuliert. Insulin selbst unterdrückt ebenfalls die Bereitstellung von Glucose aus der Leber. Beide Vorgänge verhindern nach Nahrungsaufnahme eine weitere Erhöhung des Blutzuckerspiegels. Die enterale Aufnahme von Glukose führt außerdem zur Sekretion von Inkretinen, z.B. des Glucagon-like-peptide 1 (GLP-1), welches die glukoseabhängige Insulinsekretion und Glukagonsuppression unterstützt. Inkretine gelten als 
große Innovation in der Behandlung von Diabetes, da sie ausschließlich in Anwesenheit von Glukose, also bei Nahrungsaufnahme, die Insulinfreisetzung anregen. So können Hypoglykämien vermieden werden (Nolan et al. 2011); Kapitel 2.3 beschreibt ausführlich die Therapieoptionen.

Diabetes mellitus Typ 2 ist eine multifaktorielle Erkrankung, die Ätiologie zwangsläufig komplex. Die Ursachen des Diabetes Typ 2 lassen sich in drei große Gruppen einteilen (Nolan et al. 2011; Räisänen et al. 2006):

- Evolution und Genetik

- Umwelt in frühen Lebensphasen und epigenetische Programmierung

- Lebensstil und Umweltfaktoren im weiteren Lebensverlauf.

Chronischer Energieüberschuss gilt in vielen Veröffentlichungen als der entscheidende Faktor in der Entwicklung eines Diabetes Typ 2; allein das Übergewicht oder ein erhöhter Körperfettanteil scheinen in gewissem Maße eine Insulinresistenz zu verursachen. Laut World Health Organisation (2013) ist Diabetes Typ 2 ,largely the result of excess body weight and physical inactivity". In einem deutschen Standardlehrbuch der Inneren Medizin wird betont: „Die Mehrzahl der Erkrankungen entwickelt sich auf dem Boden eines metabolischen Syndroms (= Wohlstandssyndrom)" (Herold 2014, S. 718). ${ }^{1}$ Weiterhin heißt es dort: „Überernährung mit Adipositas und Bewegungsmangel sind die entscheidenden Manifestationsfaktoren des Typ 2-Diabetes mellitus! Ca. 80 $\%$ der Typ 2-Diabetiker sind übergewichtig" (ebd.).

Mehrere Studien zeigen eine hohe Prävalenz von Übergewicht und Adipositas bei Menschen mit Diabetes Typ 2 (Al-Sharafi und Gunaid 2014; Daousi et al. 2006; Thomas et al. 2006). Eine Metaanalyse von 9 Studien durch Guh et al. (2009) konnte eine statistisch signifikante Assoziation von Übergewicht mit dem Auftreten von Diabetes Typ 2 darstellen.

Doch warum gibt es auch viele Menschen, die trotz erheblicher Überernährung nicht an Diabetes Typ 2 erkranken, fragen sich z.B. Nolan et al. (2011). Plausibel erscheint

\footnotetext{
${ }^{1}$ Das metabolische Syndrom gehört der Nationalen Versorgungsleitlinie zu Typ-2-Diabetes (Bundesärztekammer (BÄK), Kassenärztliche Bundesvereinigung (KBV), Arbeitsgemeinschaft der Wissenschaftlichen Medizinischen Fachgesellschaften (AWMF) 2013, S. 20) zufolge zu den "manifestationsfördernden Faktoren des Typ-2-Diabetes" und ist charakterisiert durch "abdominale Adipositas (Taillenumfang: Männer $>94 \mathrm{~cm}$, Frauen $>80 \mathrm{~cm}$ ), Insulinresistenz, Hyperinsulinämie, gestörte Glukosetoleranz, Dyslipoproteinämie, Albuminurie, Hypertonie (Albuminurie nur in der Definition der WHO)."
} 
die Erklärung, dass bei Menschen mit einer günstigen Prädisposition der chronische Energieüberschuss durch Erweiterung subkutanen Fettgewebes kompensiert werden kann. Dabei wird einer schädlichen Einlagerung von Fett in den inneren Organen Herz, Leber, Skelettmuskel, Pankreas - vorgebeugt, die die pathophysiologische Grundlage des Diabetes Typ 2 zu sein scheint (Nolan et al. 2011; Stefan 2008).

Eine genetische Disposition und familiäre Vererbung der Krankheit gilt als nachgewiesen (Brunetti et al. 2014). Das durch Forschung zunehmende Wissen über einzelne genetische Faktoren ist dabei eher für das Verständnis der Pathophysiologie von Bedeutung, als für die Abschätzung des individuellen Erkrankungsrisikos im Sinne einer „personalized medicine“ (Rathmann et al. 2013). Zwillingsstudien weisen auf eine Vererbung von $>50 \%$ hin (Nolan et al. 2011; Medici et al. 1999; Poulsen et al. 1999). Diesen Ergebnissen gegenüber ist jedoch Vorsicht geboten (Hopper 1999), da auch der intrauterinen und postnatalen Umgebung und Ernährung Einfluss zugeschrieben wird (Poulsen et al. 2009). Hohe Evidenz wird der These beigemessen, dass Epigenetik und Umweltfaktoren in der fetalen Phase, z.B. eine hyperglykäme intrauterine Umgebung, mit der Anfälligkeit für Diabetes verknüpft sind. Schwangerschafts- oder konditioneller Diabetes der Mutter werden als Risikofaktor angenommen. Eine Störung der neurohormonalen Mechanismen, die Appetit und Bewegungsantrieb erheblich kontrollieren, hat sich als stark erblich und durch die Umwelt in frühen Lebensphasen bedingt herausgestellt. Übergewicht kann eine Folge dieser Störung sein (Nolan et al. 2011).

Chronischer Energieüberschuss kann bei Menschen mit bestimmter genetischer und epigenetischer Disposition nicht von den B-Zellen des Pankreas kompensiert werden. Die daraus resultierenden hohen Blutzuckerspiegel beschleunigen die Schädigung der B-Zellen zusätzlich. Die fragile Homöostase des Energiehaushaltes wird unkontrollierbar. Durch eine zusätzliche Fettstoffwechselstörung lagern sich überschüssige Nährstoffe statt im subkutanen Fettgewebe in den Organen an (Leber, Muskeln, Herz, Ovarien) und schädigen diese durch Aktivierung einer Entzündungsreaktion. Die Organe werden nicht mehr von Insulin beeinflusst und können den Zucker nicht aufnehmen. Auch die Leber entwickelt eine Insulinresistenz, sodass die körpereigenen Glukoseproduktion nicht verhindert wird und lang anhaltende Hyperglykämien resultieren (Nolan et al. 2011). 
Aus der öffentlichen Betonung einzelner Einflussfaktoren dieser auffallend komplexen Ätiologie ergeben sich ethische und praktische Konsequenzen, z.B. wenn sich die Gesundheitspolitik trotz differenzierter wissenschaftlicher Debatte auf einzelne Ursachen, z.B. einen bestimmten Lebensstil beschränkt (Räisänen et al. 2006).

\subsection{Therapieoptionen}

In der Therapie spielt die Senkung des Blutzuckerspiegels eine fundamentale Rolle, da sie nachgewiesenermaßen durch mikroangiopathische Veränderungen hervorgerufene Komplikationen des Diabetes Typ 2 reduziert, wie große Studien zeigen (Matthaei et al. 2009; s. a. The ADVANCE collaborative group 2008; UK Prospective Diabetes Study (UKPDS) Group 1998a). Der Vorteil einer intensiven Blutzuckersenkung wird angezweifelt, seit die ACCORD-Studie (The Action to Control Cardiovascular Risk in Diabetes) in der Studiengruppe mit dem Ziel eines HbA1c-Wertes von 6,0\% nach 3,5 Jahren Studienlaufzeit wegen ungeklärter Erhöhung der Mortalität abgebrochen wurde. Hypoglykämien und Gewichtserhöhung um mehr als $10 \mathrm{~kg}$ Körpergewicht traten häufiger in dieser intensiv behandelten Gruppe auf als in der Vergleichsgruppe, die eine Standardtherapie erhielt. Das Risiko größerer kardiovaskulärer Ereignisse war nicht signifikant erniedrigt (The Action to Control Cardiovascular Risk in Diabetes Study Group 2008). In der ADVANCE-Studie (Action in Diabetes and Vascular Disease: Preterax and Diamicron Modified Release Controlled Evaluation), die ebenfalls den Nutzen einer starken medikamentösen Reduktion der Blutzuckerspiegel gegenüber Standardtherapie verglich, wurde dagegen keine erhöhte Mortalität in der Gruppe mit intensiver Therapie beobachtet (The ADVANCE collaborative group 2008). Auch hier wurden aber häufiger schwere Hypoglykämien beschrieben (ebd.). Es ist seitdem akzeptiert, dass nicht bei jedem Menschen mit Diabetes Typ 2 ein (fast) normaler Blutzuckerspiegel erreicht werden kann und das dieses Ziel gegenüber schweren Nebenwirkungen, wie lebensgefährlichen Hypoglykämien abzuwägen ist (Terry et al. 2012) dennoch ist eine gute Blutzuckereinstellung nach wie vor ein wichtiges Ziel der Therapie (Bailey et al. 2013).

In den Nationalen Versorgungsleitlinien zur Therapie des Diabetes Typ 2 (Bundesärztekammer (BÄK), Kassenärztliche Bundesvereinigung (KBV), Arbeitsgemeinschaft der Wissenschaftlichen Medizinischen Fachgesellschaften (AWMF) 2013, S. 23) wird „zur 
Prävention von Folgekomplikationen ein $\mathrm{HbA1c}$-[Ziel]Korridor von $6,5 \%$ bis $7,5 \%$ unter Berücksichtigung der individualisierten Therapieziele" empfohlen. Die Praxisempfehlungen der Deutschen Diabetes Gesellschaft (DDG) und der Deutschen Gesellschaft für Innere Medizin (DGIM) sehen eine individuelle Vereinbarung von Therapiezielen bezüglich des Lebensstils, Glukosestoffwechsels, Lipidstatus, Körpergewicht und Blutdruck vor (Landgraf et al. 2013). Beispielsweise gilt bei einem Body-MassIndex (BMI) zwischen 27 und $35 \mathrm{~kg} / \mathrm{m}^{2}$ die Empfehlung einer Gewichtsabnahme um $5 \%$ und bei einem BMl von über $35 \mathrm{~kg} / \mathrm{m}^{2}$ eine Gewichtsabnahme um $10 \%$ des Körpergewichts (Landgraf et al. 2013).

Erste Stufe des Therapiealgorithmus ist die „Basistherapie“, deren Bestandteile „Schulung, Ernährungstherapie, Steigerung der körperlichen Aktivität, [und] Raucher-Entwöhnung" sind (Landgraf et al. 2013, S. 154). Ist nach 3 bis 6 Monaten der individuell vereinbarte Zielwert des HbA1c zwischen 6,5 und 7,5 \% nicht erreicht, wird die „Basistherapie" mit Metformin kombiniert. Dieses orale Antidiabetikum unterdrückt die Glukoneogenese in der Leber und erhöht die Glukoseaufnahme im Muskel- und Fettgewebe. Es hat einen günstigen Einfluss auf das Körpergewicht, birgt eine geringe Hypoglykämiegefahr und verringert nachweislich das Risiko makrovaskulärer Komplikationen (Matthaei et al. 2009; Matthaei et al. 2011; UK Prospective Diabetes Study (UKPDS) Group 1998b). Als unerwünschte Nebenwirkungen treten bei etwa 20\% der Patienten gastrointestinale Beschwerden, z.B. Übelkeit und Durchfälle auf (Matthaei et al. 2011). Wird Metformin nicht vertragen oder bestehen Kontraindikationen wird ein anderes orales Antidiabetikum oder Insulin in Monotherapie eingesetzt (Landgraf et al. 2013). Beträgt der HbA1c nach weiteren 3 bis 6 Monaten mehr als der Zielwert wird in einer dritten Stufe eine Zweifachkombination von Antidiabetika, vorzugsweise mit Metformin, empfohlen. Den Einsatz von Sulfonylharnstoffen, welche die Insulinfreisetzung stimulieren, bewerten DDG und DGIM aufgrund von möglichen Nebenwirkungen wie Gewichtszunahme und mitunter letalen Hypoglykämien bei vielen Menschen als „inakzeptabel“ (Landgraf et al. 2013, S.152). Die Gefahr der Unterzuckerung besteht auch bei Gliniden, die ebenfalls kurzzeitig die Insulinsekretion stimulieren. Viel versprechend sind Ansätze mit DPP-4-Inhibitoren (z.B. Sitagliptin), welche das Enzym Dipeptidyl-Peptidase 4 (DPP-4) hemmen, das Inkretine (z.B. GLP-1) deaktiviert. So wird die Wirkung dieser Hormone erhöht. GLP-1 fördert nur bei hohem Blutzuckerspiegel die 
Ausschüttung von Insulin (vgl. Kap. 2.2, S.). So gibt es bei Anwendung der DPP-4Hemmstoffe nicht die Gefahr der Hypoglykämien. Auch der Einsatz von Insulin kommt bei Stufe 3 des Therapiealgorithmus in Betracht. Wird auch unter einer Zweifachkombination nicht der gewünschte $\mathrm{HbA1c}$ erreicht, ist die vierte und letzte Eskalationsstufe mit intensivierter Insulintherapie zusätzlich zu oralen Antidiabetika vorgesehen (Landgraf et al. 2013).

Als entscheidend gilt gerade bei einem beginnenden Diabetes Typ 2 die Veränderung solcher Lebensgewohnheiten, die das Risiko des Fortschreitens der Erkrankung erhöhen. Bei erfolgreicher Gewichtsreduktion, Umstellung der Ernährung und Erhöhung der körperlichen Aktivität kann in vielen Fällen zu Beginn auf die medikamentöse Therapie verzichtet bzw. die Medikamentengabe verringert werden. Eine finnische Studie gab Hinweise dafür, dass Diabetes Typ 2 durch Lebensstilmodifikationen verhindert werden kann (Tuomilehto et al. 2001). Die von der DDG konsentierten umfangreichen „Evidenz-basierten Ernährungsempfehlungen zur Behandlung und Prävention des Diabetes mellitus" (Toeller 2005) beziehen sich in ihrer Forderung nach Gewichtsnormalisierung unter anderem auf eine schwedische Studie, in der erhöhte Aktivität und Ernährungsumstellung metabolische Parameter wie Blutdruck, Glukosetoleranz und Lipidstatus verbesserten (Eriksson und Lindgärde 1991).

Menschen mit Diabetes müssen selbst aktiv werden. Mithilfe einer radikalen Kalorienreduktion soll Diabetes sogar "heilbar" sein (Lim et al. 2011). Bei übergewichtigen Versuchspersonen, bei denen nicht länger als vier Jahre zuvor Diabetes diagnostiziert worden war, gelang durch eine achtwöchige tägliche Minderung der Energiezufuhr auf $600 \mathrm{kcal} /$ Tag eine Normalisierung sowohl der B-Zellfunktion als auch der Insulinsensibilität der Leber und damit des Blutzuckerspiegels. Daraus wurde der Schluss gezogen, dass Diabetes Typ 2 vor allem Resultat der Verfettung von Leber und Bauchspeicheldrüse sei. Allerdings war bei drei der Untersuchten bei der Nachuntersuchung nach zwölf Wochen erneut eine diabetische Stoffwechsellage aufgetreten, sodass die Frage der Langzeitwirkung noch zu untersuchen ist. Dennoch könnten bzw. werden Ergebnisse dieser und ähnlicher Studien einen großen Druck auf Erkrankte ausüben: Fasten als Selbstheilung! 


\subsection{Diabetes als psychosoziales Problem - Selbstmanagement als Ant- wort}

Unabhängig von den gerade geschilderten Heilungserfolgen, gilt Diabetes als eine chronisch progrediente Erkrankung (Matthaei et al. 2011). Waren ursprünglich massive und nicht aufzuhaltende Gesundheitsschäden logische Folge einer solchen Erkrankung, lastet heute - auch wenn sich die Therapien verbessert haben - auf dem kranken Menschen selbst die Verantwortung, eine Krankheit zu „managen“, die niemand sonst kontrollieren oder heilen kann (Redman 2007).

Trotz der vielfältigen Behandlungsmöglichkeiten werden die metabolischen Therapieziele oft nicht erreicht (Bailey und Kodack 2011; Braga et al. 2012) und sind Komplikationen häufig (Centers for Disease Control and Prevention 2011). Als großes Problem in der Diabetesversorgung gilt die Non-Adherence bezüglich der Medikamenteneinnahme, also die mangelnde Therapietreue ${ }^{2}$ (Bailey und Kodack 2011; Nasseh et al. 2012). Die dadurch entstehenden, vermeidbaren direkten Kosten, d.h. ohne die durch Krankenhausaufenthalte verursachten Kosten durch Arbeitsausfälle und Verminderung der Produktivität miteinzubeziehen, werden z.B. für die USA im Jahr 2010 auf 105,8 Mrd. Dollar insgesamt und 453 Dollar pro Person geschätzt (Nasseh et al. 2012).

Neben der pharmakologischen Behandlung gilt die Lebensstiländerung - Gewichtsabnahme, Erhöhung der körperlichen Aktivität und Reduktion von Genussmitteln - als ebenso effektives wie unerlässliches Mittel zur Prävention und Behandlung von Diabetes Typ 2 (Centers for Disease Control and Prevention 2011; Matthaei et al. 2011). Wie es gelingt, einen solchen gesunden Lebensstil langfristig umzusetzen, gehört weiterhin zu den wichtigen Themen der Diabetes- und Ernährungsforschung (Magkos et al. 2009).

Beides, die Medikamenteneinnahme und die Lebensstiländerung betreffen den privaten Lebensbereich und Altag kranker Menschen. Häufiger und stärker als bei vielen anderen Krankheiten, ist der einzelne Mensch - nicht das medizinische Personal - für

\footnotetext{
2 Der Begriff Compliance wurde weitestgehend durch den Begriff Adherence ersetzt, der statt eines Befolgens der ärztlichen Vorgaben ein stärkere Beteiligung von Kranken an therapeutischen Entscheidungen beschreiben soll. Weiterhin wurde die Aufgabe der Begriffe Compliance und Adherence zugunsten einer angestrebten kollaborativen Arzt-Patient-Beziehung als gleichberechtigte Partnerschaft gefordert; häufig mit dem Begriff Concordance überschrieben (Chatterjee 2006).
} 
die Therapieumsetzung zuständig - und er erlebt auch als einziger die vielfältigen Konsequenzen (Anderson und Funnell 2000). Entscheidungen über Krankheitsverhalten werden auch nicht nur einmal von Menschen mit Diabetes getroffen und gelten dann als unveränderlich. Vielmehr sind täglich Entscheidungen zu treffen und immer wieder Neuanpassungen erforderlich (Paterson et al. 2001). Aktives Selbstmanagement ist daher zum Schlüsselbegriff geworden. Es umfasst neben gesunder Ernährung, Bewegung und regelmäßiger Einnahme der Medikamente auch die selbstständige Überwachung des Blutzuckers, eigenständige Medikamentenanpassung und Einhalten von Untersuchungsterminen (Harvey und Lawson 2009; Peyrot et al. 2005) und soll Menschen ermutigen, Verantwortung für ihre Behandlung zu übernehmen.

Da der entscheidende Faktor für den Behandlungserfolg der kranke Mensch selbst zu sein scheint, wurde das Krankheitsverhalten intensiv untersucht. Viele Forschungsgruppen haben sich mit der Frage beschäftigt, welche Hindernisse aus Sicht der Erkrankten der erfolgreichen „Selbstbehandlung“ von Diabetes entgegenstehen und wie man diese reduzieren kann. Jerant et al. (2005) identifizierten in Fokusgruppen mit Menschen, die an Interventionsprogrammen zur Verbesserung von Selbstmanagement bei chronischen Krankheiten teilnahmen, folgende innere und äußere Barrieren für ein erfolgreiches Selbstmanagement: finanzielle Probleme, Depression, Schmerzen, Fatigue, Gewichtsprobleme, fehlende Motivation zu Bewegung und Sport, schlechte Arzt-Patient-Kommunikation und eine geringe Unterstützung durch die Familie.

Selbstmanagement wird der DAWN-Studie (Diabetes Attitudes, Wishes and Needs) zufolge entscheidend dadurch erschwert, dass viele Menschen mit Diabetes psychosoziale Probleme haben, z.B. Ängste vor Komplikationen und Sorgen über eine Zukunft mit Diabetes (Peyrot et al. 2005). Veränderung der Stimmung und Angststörungen betreffen vergleichsweise häufiger Menschen mit Diabetes (Lin et al. 2008). Es gibt außerdem Hinweise auf eine Wechselwirkung von Hyperglyklämien und Depression; die Behandlung einer Depression ist mit verbesserten Blutzuckerlangzeitwerten assoziiert (Lustman et al. 2000).

In einer ethnographischen Studie mit Interviews und Feldbeobachtungen erkannten Hinder und Greenhalgh (2012) drei Ebenen, auf denen der Erfolg des Selbstmanagements beeinflusst wird: auf der Makro-Ebene durch die vorherrschende ökonomische 
Lage und kulturelle Normen, auf der Meso-Ebene durch Beziehungen und Rollenerwartungen in der Familie und am Arbeitsplatz sowie die persönliche finanzielle Lage und den Zugang zur Gesundheitsversorgung, auf der Mikro-Ebene durch die persönlichen Fähigkeiten. Selbstmanagement bedeutet für Menschen mit Diabetes harte Arbeit, die viel Zeit in Anspruch nimmt. Praktische und kognitive Aufgaben umfassen z.B. Planung der Mahlzeiten und selbstständige Blutzuckerkontrollen; sozio-emotionale Aufgaben bestehen darin, die Krankheit psychisch zu bewältigen, sich mit Ratschlägen und Erwartungen von anderen auseinanderzusetzen und sich Zugang zu gesundheitlicher Versorgung zu verschaffen (Hinder und Greenhalgh 2012).

Während die bisher vorgestellten Studien eine Exploration möglicher Hindernisse eines erfolgreichen Selbstmanagements - eingeschlossen gesellschaftlicher Faktoren zum Thema hatten, ist darüber hinaus die tiefergehende Beschäftigung mit individuellen Krankheitskonzepten ein großes Forschungsfeld. Erklärungen und Deutungen eines Menschen bezüglich seiner Erkrankung sollen das Selbstmanagement in besonderem Maße prägen. Sie sind Gegenstand des folgenden Kapitels.

\subsection{Krankheitskonzepte - ein integrales Modell}

Krankheitskonzept ist ein in der sozialwissenschaftlichen und medizinischen Literatur viel verwandter, aber nicht eindeutig definierter Begriff. Krankheitsvorstellung, subjektive Theorie/Vorstellung/Konzeption von Krankheit, Krankheitsbegriff und Krankheitsmodell (im Englischen: personal understanding, explanatory model, illness perception, theory of disease) sind ähnliche, aber nicht unbedingt deckungsgleich eingesetzte Ausdrücke (Kreher 2009).

Linden et al. (1988, S. 35) definierten den Begriff Krankheitskonzept als

\footnotetext{
„Summe aller Meinungen, Deutungen, Erklärungen und Vorhersagen eines Menschen hinsichtlich Störungen seines Gesundheitszustandes [...]. Krankheitskonzepte sind Ausdruck des kulturellen Hintergrundes eines Patienten, seiner Weltanschauung, seines Wertesystems, seines Lebensstils und seiner persönlichen Lebenserfahrung [und] sind bedeutungsvoll im Hinblick auf die Behandlungseinwilligung und die Mitarbeit von Patienten."
}

Diese Definition impliziert, neben einer durch äußere Faktoren wie Gesellschaft und Kultur bedingten Vorstellung von Krankheit, einen Zusammenhang zwischen Krankheitskonzept und krankheitsbezogenem Verhalten. Sowohl Therapierte als auch Therapierende entwickeln Krankheitskonzepte, gehen aber unterschiedlich damit um. 
Während ärztliche Konzepte vom Ideal rationaler Wissenschaft geprägt sind, sind Konzepte und Strategien von Erkrankten von dem Ziel bestimmt, ihren Alltag zu bewältigen und „medizinisches Wissen entlang der sinnhaften Eigenlogik ihrer Altagsmodelle zu integrieren." Krankheitskonzepte beider Gruppen weisen eine hohe „Veränderungsstabilität" auf, d.h. sie sind nicht leicht durch Gegenargumentationen zu verändern (Kreher 2009, S. 31-32).

Kleinman (1988) charakterisierte Erklärungsmodelle (explanatory models) als Vorstellungen, die Kranke, Familien und Behandelnde von einer ganz spezifischen Krankheitsepisode haben. Diese können als Rechtfertigungen für Handlungen dienen und sind eher dynamischer als feststehender und theoretischer Natur. Auch Lawton et al. (2008) beschrieben Krankheitskonzepte als prozesshaft. Sie zeigten für Menschen mit Diabetes, wie diese über die Zeit hinweg unterschiedliche Ursachen der Krankheit in den Vordergrund stellten, um krankheitsbezogene Entscheidungen zu legitimieren. Dabei müssen Krankheitskonzepte nicht immer den Handlungen vorausgehen, sondern können sich auch durch Erfahrungen mit der Krankheit verändern.

Für Kleinman (1988) sind Erklärungsmodelle zwar als kognitive Systeme vorstellbar, sind aber in starke Emotionen gebettet und eher schwerlich offen auszusprechen. Der Arzt oder die Ärztin sollte die Erklärungsmodelle der kranken Person und der betroffenen Familie adressieren und Konflikte mit dem eigenen Erklärungsmodell offen ansprechen. Fehlende Kommunikation über Krankheitskonzepte kann die therapeutische Beziehung und die kommunikative Basis der ärztlichen Versorgung gefährden.

Während Kleinman die Forschung zu Krankheitskonzepten in den Sozialwissenschaften geprägt hat, entwickelte Leventhal für die Psychologie das bis heute kaum veränderte „Common-Sense Self-Regulation Model“ (Cohen et al. 1994). Leventhal wies die damals vorherrschende Theorie zurück, dass Angst vor einer Lebensbedrohung die stärkste Motivation für gesundheitsfördernde Handlungen sei. Er entwarf ein Modell, in dem als Reaktion auf eine Bedrohung ununterbrochen sowohl emotionale als auch kognitive Prozesse parallel ablaufen, Strategien erarbeitet und einsetzt werden und Konsequenzen bewertet werden, die wiederum Einfluss auf die Krankheitskonzepte haben (Leventhal 1971). Dieses Modell hebt den Erfahrungsprozess im Krankheitsverlauf bei der Entwicklung von Krankheitskonzepten hervor. Erkrankte könnten ihre 
Konzepte im Sinne eines konstruktiveren Verständnis adaptieren und zur aktiven Problemlösung einsetzen (Harvey und Lawson 2009).

Von Leventhals Modell ausgehend wurde unter anderem untersucht, wie Krankheitskonzepte die Medikamenten-Compliance (vgl. Fußnote 2, S. 10) beeinflussen (Meyer et al. 1985). Arterielle Hypertonie ähnelt Diabetes Typ 2 aufgrund der fehlenden Symptomatik und der Notwendigkeit, über einen langen Zeitraum hinweg Medikamente einzunehmen - meist ohne einen spürbaren Effekt. Meyer et al. (1985) befragten Menschen mit Bluthochdruck gezielt zu ihren Vorstellungen von Ursachen, Mechanismen und Konsequenzen der Erkrankung und zu ihrer Compliance. Ergebnisse dieser Untersuchung bestärkten die Vorannahme der Forschungsgruppe, dass Krankheitskonzepte nicht aus dem Nichts entstehen, sondern von früheren Krankheitserfahrungen geprägt sind. Aufgrund der Erfahrung mit Krankheiten in frühster Kindheit, z.B. Schnupfen oder einem aufgeschlagenen Knie, erwarten Menschen, dass eine Krankheit - auch arterielle Hypertonie - von Symptomen begleitet wird, die durch eine Behandlung bis hin zur Heilung vermindert werden. Meyer et al. beobachteten, wie Patienten mit wachsender Erfahrung auch ihren Bluthochdruck in Form von spezifischen Symptomen wahrnehmen konnten und dadurch die Krankheit kontrollierten. Sie sahen dies in dem Wunsch der Kranken begründet, abstrakten Konzepten, wie dem label Bluthochdruck konkrete Zeichen zuzuordnen. Krankheitskonzepte werden also aus zwei Komponenten konstruiert: aus konkreten Symptomen und abstrakten Konzepten von Verlauf, Ursachen und Pathophysiologie. Für die von Meyer et al. befragten Menschen stand das konkrete Empfinden blutdruckspezifischer Symptome dem ärztlichen Konzept von Bluthochdruck als einer nicht spürbaren Erkrankung entgegen. Sie verinnerlichten das Konzept, das innen von ärztlichem Personal und Medien vermittelt wurde, behielten aber davon unabhängig ihre persönlichen Vorstellungen bei. Im Bewusstsein dieser Inkongruenz erwähnten Menschen ihre eigenen Konzepte dem Arzt oder der Ärztin gegenüber aber meist nach einiger Zeit nicht mehr.

Der Einfluss gesellschaftlicher Normen und Rollenzuweisungen auf Krankheitskonzepte wird unter anderem in dem Zusammenhang von Geschlechterrollen und Diabetes deutlich, den Broom und Treacher (2003) thematisierten. In einer Übersicht über das in australischen Printmedien (v.a. in der Zeitschrift „Diabetes Conquest“) für das 
Laienpublikum gezeichnete Bild der Krankheit kritisieren sie die traditionelle Repräsentation der Geschlechter: Die Unterschiede in der Krankheitserfahrung wird für Frauen auf Probleme der Reproduktion reduziert, z.B. durch Empfehlungen, den Blutzucker im Falle einer Schwangerschaft besonders gut zu kontrollieren, für Männer ausschließlich auf das Problem der mit Diabetes assoziierten erektilen Dysfunktion. Die Medien sind von der Vorstellung der Frau als einer für das leibliche Wohl der Familie sorgenden Mutter und des sportlich und beruflich erfolgreichen Mann geprägt. Die Beschränkung auf traditionelle Rollen kann bedeuten, dass sich von den Publikationen ausschließlich Menschen angesprochen fühlen, die sich mit konventionellen Geschlechterrollen identifizieren. Wissenschaftliche und medizinische Autoritäten dominieren die Medien und erklären auf diese Weise ganz bestimmte Modelle von Maskulinität und Femininität für gültig. Das ist für das Ende des 20. Jahrhundert (die Übersicht umfasst Veröffentlichungen von 1996-2001) bemerkenswert konservativ.

Dass diese traditionelle Rollenverteilung aber tatsächlich für viele Menschen das alltägliche Leben mit Diabetes beeinflusst, zeichnete sich in einer 2010 von Broom und Lenagh-Maguire veröffentlichten qualitativen Studie ab. Männliche Studienteilnehmer thematisierten häufig Arbeitsanforderungen als Hindernisse, den Diabetes zu kontrollieren, was die starke Identifizierung mit dem Beruf widerspiegelt. Oft würde den Ehefrauen die Verantwortung für die Krankheit zugesprochen, da die Frau traditionell für die Zubereitung von Essen und für die Gesundheit der ganzen Familie zuständig sei. Weibliche Befragte beschrieben häufig Doppelbelastungen durch Erwerbstätigkeit und Arbeit für die Familie.

Ausbrüche aus diesen konventionellen Verhaltensmustern zeigten jedoch, dass die Annahme, dass alle Menschen mit Diabetes diese traditionellen Geschlechterrollen übernähmen, falsch ist. Die grundsätzliche Bestärkung in diesen Konventionen sei also nicht hilfreich für das Krankheitsmanagement, so Broom und Lenagh-Maguire. Statt die Behandlung von Diabetes auf die von Geschlechterrollen geprägten sozialen Bereiche Essen und Sport zu konzentrieren, kann ein komplexeres Verständnis des Zusammenhangs von Geschlecht und chronischer Krankheit die Versorgung möglicherweise verbessern.

Krankheitskonzepte scheinen zum einen die Krankheitsbewältigung im psychologischen Sinne zu beeinflussen, sind zugleich aber auch ein sich wandelndes Ergebnis 
von Integration der Krankheit in das Altagsleben der Erkrankten. Sie sind also kritischer Faktor im praktischen Umgang mit Krankheiten (Meyer et al. 1985). Von besonderer Bedeutung scheinen Unterschiede zwischen ärztlichen Krankheitskonzepten und denen der Erkrankten zu sein, da sie Konfliktpotential bergen (Kleinman 1988). Nicht außer Acht zu lassen ist der starke Einfluss sozialer Rollenerwartungen und gesellschaftlicher Normen auf Krankheitskonzepte. Ähnlich der immer gestellten Frage nach guter und schlechter Krankheitsbewältigung (Pfeffer 2010), werden auch Krankheitskonzepte hinsichtlich ihres Nutzens für den Behandlungserfolg befragt.

\subsection{Krankheitskonzepte im Spannungsverhältnis}

Das Interesse an der Erforschung der Krankheitskonzepte gerade von Menschen mit chronischen Krankheiten hat seit der Erkenntnis, dass diese das Krankheitsverhalten entscheidend beeinflussen, stetig zugenommen. Insbesondere ging es darum, zu verstehen, wie sich die Krankheitskonzepte der Kranken von biomedizinischen, „ärztlichen" Krankheitskonzepten, unterscheiden.

Hörnsten et al. veröffentlichten 2004 die Ergebnisse einer qualitativen Studie zum „Personal Understanding“ von Menschen mit Diabetes Typ 2 in Schweden. Auch sie nahmen an, dass Konflikte in der Arzt-Patient-Beziehung oft Resultat des unterschiedlichen Krankheitskonzeptes sind. Behandelnde und Behandelte leben bezüglich ihrer Krankheitserfahrung in verschiedenen Welten (s.a. Toombs 1992). Daher stehen die Therapieziele mitunter im Widerspruch. „Personal Understanding“ wird hier als ein Geflecht von Gedanken, Gefühlen, Erklärungen und Verständnis beschrieben, das den Kranken hilft, die Krankheitserfahrung sinnvoll in ihre Lebenswelt einzuordnen. Die Arbeitsgruppe führte narrative Interviews durch, bei denen sie mit einer erzählgenerierenden Eingangsfrage „When did you get the diabetes“ Informationen zur Entwicklung eines Diabetes und zum Umgang mit der Erkrankung erhielt. Nach Durchführung einer qualitativen Inhaltsanalyse ließen sich Krankheitskonzepte durch Kategorien charakterisieren: z.B. wie viel Raum Menschen ihrer Krankheit innerhalb des Lebens einräumen und welche Pläne und Hoffnungen sie für die Zukunft haben. Zwischen den Kategorien "Meaning of the Diagnosis" und "Integration of the Illness" unterschieden Hörnsten et al. (2004) nachdrücklich. Erstgenannte Kategorie setzt sich mit den Emotionen und Rollenkonflikten auseinander, die die Diagnose mit sich bringt, z.B. ob die 
betroffene Person akzeptiert, dass sie nun bestimmte Regeln einhalten soll oder weiterlebt wie zuvor. Sie umfasst auch die mit der Diagnosestellung verbundenen Gefühle wie Scham oder Angst vor Stigmatisierung. Die Kategorie "Integration of the IIIness“ dagegen befasst sich mit der meist einige Zeit nach Diagnosestellung beginnenden Eingliederung der Erkrankung in den Alltag und den Gefühlen gegenüber einem Leben mit der Erkrankung. Ärztinnen und Ärzte verstehen oft den Unterschied nicht, da für sie die Diagnosestellung die Lösung des Problems ist, während die Erkrankten bei der Diagnosestellung am Anfang eines Problems stehen, an das sie sich erst gewöhnen und einen Umgang mit den neuen Anforderungen erlernen müssen.

In dieser, wie in vielen weiteren Studien wurde herausgestellt, dass sich gerade die Therapieziele von Erkrankten grundsätzlich von denen des medizinischen Personals unterscheiden. Ziel von Menschen mit Diabetes ist nicht in erster Linie das Erreichen der metabolischen Zielwerte entsprechend der ärztlichen Absicht, sondern ein Leben in „Balance“ (Gillibrand and Flynn 2001; Kneck; Paterson und Thorne 2000). Kneck (2001) versteht "Balance" als Ergebnis der erfolgreichen Integration von Diabetes in den Alltag, ohne das Gefühl zu haben, dass die Krankheit den Alltag bestimmt. Paterson und Thorne (2000) beschrieben einen Lernprozess, in dem Menschen zu Experten ihrer Krankheit werden. Mit diesem Wechsel ist verknüpft, dass sie sich nicht mehr als von der Krankheit kontrolliert empfinden, sondern die Auswirkungen der Krankheit kontrollieren.

Zwischen dem Krankheitsverständnis von Patientinnen und Patienten und der Art der Gesundheitsversorgung bestehen laut Lawton et al. (2005) enge Wechselwirkungen. So kann z.B. die Behandlung in einer Diabetesklinik den Eindruck verstärken, dass Diabetes eine schwerwiegende Erkrankung ist, während Menschen, die allgemeinmedizinisch ambulant versorgt werden, daraus den Schluss ziehen können, dass ihre Lage nicht so ernst ist. Die Erwartungen, die Menschen an die Gesundheitsversorgung stellen, sind aber umgekehrt auch durch das persönliche Krankheitsverständnis beeinflusst. Räumt jemand der Krankheit wenig Platz in seinem Lebenskonzept ein, kann er oder sie geneigt sein, eine fachärztliche Behandlung als unnötig zu bewerten. Eine große Herausforderung besteht Lawton et al. zufolge darin, grade symptomlose Kranke davon zu überzeugen, dass Diabetes eine ernst zu nehmende Erkrankung ist. 
Thorne et al. (2000) beschrieben, dass Menschen mit chronischen Krankheiten meist eine globale und ganzheitliche Erklärung ihrer Krankheit haben, während sie über die biomedizinisch wissenschaftliche Ansätze hinausgehende Konzepte bei ärztlichem Personal vermissen. Die im Gesundheitssystem vorherrschende Haltung, die Kompetenz der Erkrankten anzuzweifeln, beeinflusst die alltägliche Krankheitserfahrung. Da die Professionellen die Emanzipation der Kranken nur formal akzeptieren, empfinden sie Nachfragen oft als Belästigung oder sogar als obsessiv und weisen neugierige Menschen ab. Es findet eine schwerwiegende Entfremdung in der Arzt-Patient-Beziehung statt. Mitunter stellen Ärztinnen und Ärzte, sogar wenn sie wenig Fachkompetenz in dem betreffenden Bereich besitzen, ihre Expertise über die der erfahrenen Kranken, z.B. wird die fachärztlich festgelegte Medikamenteneinstellung in anderen Fachrichtungen oft einfach ignoriert.

Dass das unterschiedliche Krankheitsverständnis von medizinischem Personal und chronisch Kranken zu Problemen in der Arzt-Patient-Beziehung führen kann, kommentierten auch die amerikanischen Forscher Anderson und Robins (1998). Aus der Akutmedizin gewohnt, dass Kranke ihre Anweisungen befolgen, fühlen sich Professionelle für das Wohlergehen dieser mehr oder minder allein verantwortlich und haben den Wunsch, dass ihr ärztliches Handeln Erfolg zeigt. Daher empfinden sie die, für eine chronische Krankheit charakteristische, Verlagerung der Einflussmöglichkeiten in die Hände der Erkrankten als Kränkung und Hilflosigkeit gegenüber ihrer eigenen Verantwortung, Menschen zu heilen. Da sie ihre Sichtweise häufig - wie alle Menschen - als die richtige betrachten, werden Erkrankte, die nicht die ärztlichen Erwartungen erfüllen, häufig mit dem Label „non-compliant“ versehen. Den Autoren zufolge ist diese „moralische“ Verurteilung selten ein Anlass für medizinisches Personal, den Gründen für ein Nichtbefolgen ihrer Ratschläge auf den Grund zu gehen. Sich Zeit zu nehmen, die Sichtweise der Betroffenen zu erfragen, schätzen die Autoren als hilfreich ein, um deren Verhalten zu verstehen. Sie meinen, dass die Behandlung von Diabetes nicht effektiv sein kann, wenn sie in ein gedankliches, finanzielles und psychologisches System gepresst wird, welches für die Akutversorgung entwickelt wurde. Nötig ist ein neues System, welches an die Versorgung chronischer Erkrankungen angepasst ist. Anderson und Funnell führten den Gedanken im Jahr 2000 weiter und wiesen die Konzepte Compliance und Adherence als dysfunktional in der Diabetesversorgung zurück, 
zugunsten des Modells einer kollaborativen Arzt-Patient-Beziehung, in welcher der kranke Mensch auf dem Weg zu einer eigenverantwortlichen Behandlung unterstützt wird.

In diesem Zusammenhang muss auch das Konzept der „mündigen Patienten“ diskutiert werden. In der gesundheitspolitischen Debatte häufig durch die Zuschreibung der Fähigkeit zur souveränen Förderung der eigenen Gesundheit und Vermeidung von Risiken idealisiert, stehen Ärtzinnen und Ärzte den, durch diesen Begriff charakterisierten, Personen zwiespältig gegenüber. Einerseits können gut informierte Kranke die ärztliche Arbeit erleichtern, andererseits werden sie als unbequeme, das Gesundheitswesen „konsumierende“ Menschen wahrgenommen, welche die ärztliche Expertise infrage stellen könnten (Dieterich 2007). Obwohl die Emanzipation der Erkrankten allgemein erklärtes Ziel ist, wird ihr Wissen - trotz langjähriger Erfahrung mit einer chronischen Krankheit - häufig nicht anerkannt (Thorne et al. 2000). Patersons (2001) über zehn Jahre alte Behauptung, dass die Übergabe von Verantwortung und Entscheidungsmacht an die Kranken bisher ein Mythos geblieben sei, dürfte auch heute gelten.

Die „moralische“, gewissermaßen „moralisierende“ Sprache in der Diabetesbehandlung beschrieben die australischen Autorinnen Broom und Whittaker (2004). In narrativen Interviews fiel ihnen auf, dass Menschen mit Diabetes Typ 2 ihre Beziehungen und die eigene Identität häufig mittels einer von Kontrolle, Überwachung, Disziplin und Verantwortung geprägten, widersprüchlichen Sprache darstellten. Letztlich wird die Identität der Erkrankten mit der Diagnose beschädigt. Diabetes wird allgemein meist als eine Erkrankung gesehen, die auf mangelnde Selbstkontrolle und Verantwortungslosigkeit gegenüber der eigenen Gesundheit zurückzuführen sei. So führt die Erkrankung zu einem Gefühl des Andersseins, der Angst vor Ausgrenzung und tatsächlicher Diskriminierung. Ein Schlüsselwort der heutigen gesundheitsbewussten Leistungsgesellschaft, in der Gesundheit als persönlicher Verdienst und eigene Verantwortung wahrgenommen werde, ist das Wort „Kontrolle“. Menschen mit Diabetes befinden sich in einem dicht gestrickten Netz der Kontrolle durch medizinisches Personal, Blutzuckermessgeräte, beratende Familienmitglieder, moralische Beurteilung durch die Gesellschaft und sich selbst. Broom und Whittaker (2004) zufolge entwickeln sie vielfältige Verteidigungsstrategien, um das Bild aufrechtzuerhalten, sie hätten sich „unter Kontrolle“ und die eigene moralische Integrität zu bewahren. 
Das Bedürfnis nach Normalität kann auch in der Vertuschung von Problemen münden: indem man sich z.B. in Gesellschaft ein Stück Torte genehmigt oder vor dem ärztlichen Kontrolltermin das eigene Blutzuckertagebuch manipuliert. Das hohe Spannungspotential zwischen medizinischem Personal und Menschen mit Diabetes zeigt sich deutlich durch die in den Patienteninterviews verwendete Sprache, die eher zu Kindern passt, die eine Regel verletzen, als zu Erwachsenen. Die von Broom und Whittaker interviewten Personen beschrieben sich selbst als "frech“ und sprachen von Nahrungsmitteln, die sie eigentlich nicht essen „dürfen“. Die Autorinnen sehen darin eine Karikatur der Art und Weise, wie medizinisches Personal mit den Erkrankten spricht. Menschen funktionieren nicht mechanisch und schlechtes Management der Blutzuckerwerte sei nicht zwangsläufig Zeichen einer falschen Selbstbehandlung. Die Behandlung des Diabetes ist eine persönliche komplexe und dynamische Aufgabe. Durch genaue sprachliche Analyse von Interviews mit Erkrankten können Probleme erkannt und Schlussfolgerungen für Änderungen in der medizinischen Praxis gezogen werden.

Parry et al. (2005) betonen, dass Menschen mit Diabetes die schwierige Nachricht begreifen müssen, dass Ursachen und Behandlungsergebnisse aber auch bei größter Anstrengung nicht vollständig in ihrer Kontrolle sind. Wo erwartete Ergebnisse eigener Bemühungen nicht eintraten und die Krankheit sich verschlimmerte, schien es den von innen interviewten Menschen mit Diabetes Typ 2, als würde die Krankheit „sich selbst widersprechen“. Der Misserfolg wurde mit persönlichem Versagen gleichgesetzt und eine Veränderung des Lebensstils als unerreichbares Ziel empfunden. Es ist wichtig, Menschen mit Diabetes darüber aufzuklären, dass die Krankheit - auch wenn sie sich "optimal" verhalten - fortschreiten kann. Zwei diskursive Konstruktionen, die sich anteilig in allen Erfahrungsberichten fanden, wurden von Parry et al. identifiziert: „Up to them" und "Down to me“. Mit "Down to me" beschrieben sie die Einstellung von Menschen, die sich selbst verantwortlich für Ursachen und Behandlung des Diabetes fühlten. Dieser Konstruktion, die sie eher mit Erfolg und einer positiven Umdeutung der Diagnose als Wendepunkt in Richtung eines "gesunden Lebens“ assoziierten, stellten Parry et al. die Konstruktion „Up to them“ entgegen. „Up to them“ - „Von denen abhängig“ - steht für den Wunsch nach Absolution von der eigenen Schuld durch das ärztliche Gegenüber und nach Abgabe von Verantwortung. Parry et al. bemerkten, dass dieser Wunsch so weit gehen kann, dass Menschen die Kontrolle aller Lebensbereiche 
an Professionelle übertragen möchten - bis hin zur Füllung des Kühlschranks mit ausschließlich „erlaubten“ Lebensmitteln (Parry et al. 2005).

Wie sich in den beschriebenen Studien zeigt, geht es in der Literatur zu Diabetes mellitus häufig um die problematische Arzt-Patient-Beziehung, besonders im Zusammenhang mit der Forderung nach einer aktiven Übernahme von Verantwortung durch die Erkrankten. Collins et al. veröffentlichten 2009 eine explorative Studie zu Strategien der Selbstbehandlung. Sie führten narrative Interviews zu alltäglichen Erfahrungen von Menschen mit Diabetes Typ 1 und 2. In der qualitativen Auswertung erkannten sie drei verschiedene Typen: „Proactive Manager", „Passive Follower" und „Nonconformist“. Die drei Typen unterscheiden sich darin, wie sie Gesundheit und Verantwortung bewerten und verschiedene Strategien in der Selbstbehandlung verfolgen. Die Forschungsgruppe leitet daraus die Notwendigkeit ab, das Behandlungskonzept daran anzupassen. Auch wenn die Kategorisierung in drei Typen die Erfahrungen der Interviewten nur unzureichend beschreibt, deuten drei Varianten immerhin die Vielfalt an, wie Menschen ihre Erkrankung verarbeiten, sodass man die Komplexität und Bandbreite des möglichen Krankheitsverhaltens erahnen kann. Ebenso wie Anderson und Funnell (2000) zweifeln Collins et al. (2009) Compliance als Erfolgskonzept an. Auch wenn die Gruppe der „Passive Follower" sich compliant, also „treu“ gegenüber einem Therapieregime verhält, kann ihr eine Schulung zur selbständigen Entscheidung über gesundheitsfördernden Maßnahmen nützen. An diesem Beispiel wird der Wandel hin zur aktuellen gesellschaftlichen Forderung an Kranke deutlich. Heute steht, statt des Gehorsams gegenüber einem festgelegten Therapieregime, die Eigenverantwortlichkeit im Mittelpunkt.

Moser et al. (2008) schätzen die Förderung der Autonomie von Menschen mit Diabetes als wichtigen Teil der patientenorientierten Behandlung ein. Autonomie definieren Kranke als Fähigkeit, ihr Leben selbst zu gestalten. Da für die Forschungsgruppe die Identifikation der Menschen mit ihrer Krankheit eine Dimension von Autonomie ist, wollten sie mithilfe einer an der Grounded Theory orientierten Analyse von narrativen Interviews diesen Aspekt der Krankheitserfahrung beschreiben. Das Ergebnis ist ein Phasenmodell der Krankheitsbewältigung: Versuche, die Krankheit zu begreifen (comprehending), Konflikt (struggling), Beurteilung (evaluating) und Meistern von Proble- 
men (mastering). Der Prozess der Identifizierung mit der Krankheit ist nicht linear, sondern zyklisch und kontinuierlich, da sich Bedingungen der Krankheit ständig wandeln. Obwohl die Menschen selbst den Prozess durchlaufen, ist es entscheidend, dass medizinisches Personal innen Therapien anbietet, mit denen sie sich zu gegebenem Zeitpunkt identifizieren können: z.B. sie in einer Phase der Beurteilung durch Aufzeigen ihrer Erfolge zu bestärken oder in einer Phase des ersten Begreifens auf ihre persönlichen Erklärungsmodelle einzugehen. Von Mosers Ergebnissen ausgehend könnte die genauere Analyse von Erklärungsmodellen interessant sein, auch wenn die Einteilung in Phasen unnötig starr wirkt.

Bei Paterson and Thorne (2000) finden wir ebenfalls ein Phasenmodell, das den Lernprozess von Menschen in Richtung einer erfolgreicher Selbstbehandlung (hier bei Diabetes Typ 1) beschreiben soll. Dabei berücksichtigt die Arbeitsgruppe jedoch, dass die vier Phasen - passive Compliance, Rebellion, naives Experimentieren und aktive Kontrolle - nicht zwangsläufig der Reihe nach durchlaufen werden. Bisweilen übertragen Menschen mit Diabetes auch nach bereits erfolgter Übernahme von aktiver Kontrolle, in bestimmten Situationen, z.B. Tod enger Angehöriger oder anderer belastender Umstände, die Kontrolle der Krankheit an Professionelle und wechseln so zwischen den beschriebenen Phasen hin- und her. Ärztinnen und Ärzte müssen also auch nach einer jahre- oder jahrzehntelang eingespielten Arzt-Patient-Beziehung neugierig und offen sein für Änderungen im Leben der Erkrankten und neue Formen der Krankheitsbewältigung.

Ahlin and Billhult (2012) hatten das Ziel, Erfahrungen von mit Lebensstiländerungen bei einer chronischen Krankheit am Beispiel Diabetes Typ 2 zu untersuchen. In der durch Studien bestärkten Annahme, dass Frauen mehr Schwierigkeiten mit solchen Veränderungen haben, wurden ausschließlich Frauen in die Arbeit einbezogen. Die Forscherinnen führten Interviews mit zehn Frauen in Schweden durch, die sie mithilfe eines „Purposive Samplings“ (s.a. Coyne 1997) auswählten, um Variationen von Alter, Krankheitsdauer, Schwere der Krankheit und Komplikationen in der Stichprobe zu gewinnen. Die narrativen Interviews wurden mithilfe einer Methode phänomenologischer Forschung nach Giorgi ausgewertet, bei der durch wiederholtes Lesen und Aufteilen der Daten in sinnhafte Abschnitte Themen identifiziert und unter einer generellen Über- 
schrift zusammengefasst wurden. Als "Essenz" der Erfahrung, den Lebensstil zu verändern erkannten Ahlin and Billhult (2012) einen andauernden inneren Kampf (,a continuous inner struggle"). Die Frauen sehen sich als Opfer der Krankheit und fühlen sich ungerecht behandelt. Dies führt zu einem sowohl äußeren als auch inneren Kampf mit den Anforderungen eines gesunden Lebenstils. Eine Frau wird zitiert, welche es ungerecht findet, selbst darauf achten zu müssen, was sie isst, während andere, die nicht Diabetes haben, tun können „was sie wollen“. Die Autorinnen entwickelten fünf Unterthemen: „The ambigous feeling of others involvement“, „Becoming a victim of pressuring demands“, „Experiencing knowledge deficits“, „Experincing an urge“ und „Finding reasons to justify not changing“. Darin beschrieben sie, wie Interviewte die Erwartungen von Mitmenschen als Argument benutzen, um einen ungesunden Ernährungsziel beizubehalten, z.B. wenn regelmäßige, gesunde Mahlzeiten nicht mit den Wünschen der Familie übereinstimmen. Die Frauen stehen unter großem innerem und äuBerem Druck, der so überwältigend sein kann, dass sie nicht motiviert sind, ihre Gewohnheiten zu ändern. Das Wissen über nötige Lebensstilveränderungen hilft nicht, die Konflikte zu verringern, die zwischen dem Bedürfnis „das Richtige“ zu tun und dem Verlangen nach bestimmten Lebensmitteln bestehen, das eine Interviewte mit einer Drogensucht verglich. Auffällig sind die vielfältigen Begründungen, mit denen die Frauen rechtfertigen, sich nicht vermehrt zu bewegen oder gesünder zu ernähren. Ahlin and Billhult (2012) deuten dies als Wunsch der Frauen, ihr Leben wie vor der Diagnose weiterzuführen. Medizinisches Personal soll diesen Kampf oder Konflikt verstehen und Frauen mit Diabetes Typ 2 mit großen Respekt behandeln. Die Autorinnen sehen darin eine Chance, diese darin zu unterstützen, die Lebensstilveränderung als Herausforderung zu betrachten, die ihre Lebensqualität und die Gesundheit verbessern kann. Diese Studie hat das Augenmerk sehr schön auf die Perspektive der Patientinnen gerichtet, wobei ein kontrastierender Vergleich zum Krankheitserleben von Männern sicher noch aufschlussreicher gewesen wäre.

\subsection{Misserfolge und Missverständnisse: Zuschreibung von Verantwort- lichkeit}

Als Grund für Behandlungsmisserfolge, z.B. sichtbar durch ein Fortschreiten der Erkrankung oder Komplikationen, galt lange der Mangel an Wissen der Menschen mit 
Diabetes über die Krankheit. So richtete sich die Hoffnung auf die Verbesserung von Schulungen. Recht bald hat sich aber herausgestellt, dass Fachwissen allein zum Gelingen des Selbstmanagements nicht ausreicht, sondern vielmehr das Krankheitsverständnis entscheidend ist (Harvey und Lawson 2009). Daneben, so kritisieren Mühlhauser und Lenz (2008), dürfen Menschen mit chronischen Krankheiten nicht aufgrund von fehlender Compliance für schlechte Behandlungsergebnisse verantwortlich gemacht werden. Sie beklagen die „non-compliance der Leistungserbringer zur Bereitstellung von angemessenen Patienteninformationen" als Ursache von Therapieversagen (S. 223). Statt evidenzbasierter Information werde oft „Medikamentenwerbung an Patienten und Bürger für rezeptpflichtige Medikamente durch die Industrie unter dem Deckmantel der Information“ ausgegeben - mit der Gefahr der „Verpflichtung von Patienten zu therapiegerechtem Verhalten“ (ebd.).

Holmström und Rosenqvist (2005) beschäftigten sich mit "Missverständnissen“ in der Behandlung von Diabetes. Sie beschrieben das Problem, dass sich trotz des großen Aufwandes zu fachgerechter Versorgung und Aufklärung weniger als die Hälfte der Behandelten in einer guten Stoffwechsellage befinde. Als Ursache für den Misserfolg sehen sie „Missverständnisse" der Menschen bezüglich ihrer Krankheit und deren Behandlung. Sie zeichneten Arzt-Patient-Begegnungen in Sprechstunden auf Video auf. Die Forscher zeigten den untersuchten Menschen mit Diabetes die Aufnahmen und befragten sie danach, ob sie die jeweilige Begegnung als gut oder schlecht empfunden hätten, um auf diese Weise Einblicke in das Krankheitsverständnis zu erhalten. Als Missverständnisse bewertete Aussagen waren z.B. die Annahmen, „Diabetes Typ 2 ist kein richtiger Diabetes“, „In der Diät ist das Wichtigste, Fett zu reduzieren“ und „Körperliche Aktivität ist gut“. Holmström und Rosenqvist hoben hervor, dass einige Kranke die Zahlen der Blutzuckerwerte nicht immer mit dem "realen Leben“ in Verbindung brachten und nicht glaubten, diese beeinflussen zu können. Bedenklich schien innen auch, dass viele die Funktion der Bauchspeicheldrüse nicht verstehen und selbst bei medikamentöser Behandlung von Komplikationen wie z.B. einer Angina pectoris nicht wahrnehmen, dass Spätfolgen bereits manifest seien. In der Studie wird betont, dass die Erkrankten auf keinen Fall als "dumm“ oder "non-compliant“ betitelt werden dürfen. Viele halten sich sogar zu strikt an das Therapieregime. Aus Sicht von Holmström und 
Rosenqvist müssen Schulungen einen Wechsel von passiver Therapietreue (Compliance) zu aktivem Selbstmanagement leisten. Interessanterweise wurden aber vier der achtzehn Studienteilnehmer gelobt, die nichts "missverstanden“ und demzufolge den medizinischen Wissensstand und die eigenen Erfahrungen integriert hätten. Maßstab für diese positive Bewertung ist dann letztlich doch medizinische Wissenschaft - und nicht ein patientenorientiertes Kriterium. Die Autoren schließen mit der Empfehlung, dass das individuelle Krankheitsverständnis erkundet werden müsse und die Kranken angeregt werden sollen, darüber nachzudenken. Die Reflexion ihrer Erfahrungen sei für diese ein wichtiger Schritt, Expertise über ihre Krankheit zu erlangen.

In dieser Studie wird deutlich, dass selbst Forschungsgruppen, die das Krankheitsverständnis von Patienten wichtig finden und wahrnehmen möchten, bewusst oder unbewusst metabolische Outcomes bzw. Lehrbuchwissen über Diabetes als Maßstab verwenden. Damit deutet sich eine Grenzziehung zwischen guten und schlechten Patientinnen und Patienten an. Selbst wenn Holmström and Rosenqvist gegenüber dem „veralteten" Konzept der Compliance ein aktives Selbstmanagement für sinnvoller zum Erreichen des gewünschten Outcomes erachten, analysierten sie die Interviews mit einer festen Vorstellung von korrekten und falschen Aussagen. Dabei möchte ich nicht die Absicht der Studie anzweifeln, die Versorgung zu verbessern und ein aktives Selbstmanagement zu fördern. Die Wortwahl "Missverständnis“ weist aber darauf hin, dass für die Autoren eine Sichtweise die Richtige ist, nämlich die der evidenzbasierten Medizin. Krankheitstheorien erscheinen dann insofern interessant, als dass Menschen mit Diabetes durch Reflexion in Richtung einer besseren Krankheitssicht korrigiert werden sollen. Daneben stellt sich die Frage, ob Holmström and Rosenqvist (2005) die in den gefilmten Sprechstunden vorkommenden Aussagen des medizinischen Personals ebenfalls zur Aufklärung von kommunikativen Missverständnissen in die Analyse der Patienteninterviews einbezogen haben.

Eine Studie aus Österreich behandelt ebenfalls Erfahrungen von Menschen mit Diabetes Typ 2 und deren Motivation zur Lebensstilveränderung (Seidl et al. 2007). Seidl et al. interessierten sich für den „Wissenstand der PatientInnen in Bezug auf ihre Krankheit“, „Probleme und Schwierigkeiten, die betreffenden PatientInnen in Bezug auf die erforderlichen Maßnahmen wie zum Beispiel Diätzubereitung und Prävention 
in ihrem Alltag erleben.“ „Besondere Beachtung“ wollten die Autorinnen „Lebensbedingungen“ widmen, „ebenso wie den Umweltfaktoren, die dazu beitragen könnten, dass die/der Einzelne die Richtlinien nicht einhält und eine Rehospitalisierung notwendig wird“ (S. 73).

In diesem Forschungsvorhaben wird deutlich, dass die Erfahrungen vor dem Hintergrund der scheinbar mangelnden Beteiligung der Kranken betrachtet werden. Neben einer themenzentrierten Auswertung führten Seidl et al. eine theoriegeleitete Auswertung der Interviews unter Einbeziehung des „sozial-kognitiven Prozessmodells gesundheitlichen Handelns nach Ralf Schwarzer" durch (Seidl et al., S.131). Dies gliedert sich in verschiedene Schritte: „Von der Wahrnehmung einer bestehenden oder potenziellen Gesundheitsgefährdung bis zur Realisierung gesundheitsfördernden Verhaltens" (ebd.). Dabei wird angenommen, dass für die Motivation zu einem bestimmten Verhalten die Selbstwirksamkeitserwartung von Bedeutung ist, also die Überzeugung der eigenen Befähigung, sich in gewisser Weise zu verhalten (s.a. Schwarzer und Jerusalem 2002; Bandura 1977). Seidl et al. beschrieben, dass sich die Interviewten in ihrer Kompetenz- oder Selbstwirksamkeitserwartung unterscheiden. Während einer der von innen Interviewten sich z.B. zutraut, „Topfenstrudel ohne Zucker“ zu backen (S. 155), sind andere der Überzeugung, z.B. wegen ihrer Berufstätigkeit nicht in der Lage zu sein, ihre Lebensgewohnheiten zu ändern.

Ingadottir und Halldorsdottir (2008) sind in ihrer Studie zum subjektiven Erleben mit Diabetes Typ 2 sicherlich hinsichtlich der Zuschreibung von Verantwortung für therapeutische Misserfolge von einem „patientenfreundlicheren“ Ansatz ausgegangen. Sie führten Interviews mit 11 Personen mit Diabetes (alle bis auf eine hatten Typ 1) in Island und explorierten Erfahrungen mit Diagnose, Behandlung und eigener Adherence und Non-Adherence. Dabei stellten sie fest, dass die Menschen sich einer widersprüchlichen Forderung ausgesetzt fühlen: "Be in charge and responsible for $y$ our life" und gleichzeitig "Do what we [health care professionals] tell you to do." (S. 615). Ingadottir und Halldorsdottir argumentieren gegen die Annahme, mangelnde Therapietreue sei irrational und unverantwortlich. Vielmehr zeigten ihre Ergebnisse, dass die Interviewten die Verantwortung für ihre Gesundheit übernehmen und dies auf komplexe Weise verhandeln. Sie betonen, dass die Menschen ernsthaft versuchten 
alles „richtig“ zu „machen“, auch wenn ihnen die Kontrolle der Laborwerte nicht immer gelänge.

\subsection{Zusammenfassung und Beurteilung}

Die „epidemieartige“ Verbreitung von Diabetes scheint trotz hochentwickelter medikamentöser Therapien nicht aufzuhalten zu sein und verursacht durch Folgeschäden hohe Kosten und individuelles Leid. In der öffentlichen Debatte gilt besonders ein ungesunder Lebensstil als relevant für die Krankheitsentstehung (Räisänen et al. 2006). Therapieziele sind klar durch metabolische Zielwerte definiert. Als problematisch gilt die Non-Compliance oder Non-Adherence bezüglich der Medikamenteneinnahme und der geforderten Veränderung des Lebensstils. Es wurde erkannt, dass die Behandlung in den Händen der Erkrankten liegt, in alltäglich neu zu treffenden Entscheidungen für ein "gesundes“ oder "ungesundes“ Verhalten. Krankheitskonzepte gelten als kritisch für das Krankheitsverhalten. In der Literatur findet sich daher eine umfangreiche Sammlung von Theorien und Modellen zu Krankheitskonzepten von Menschen mit Diabetes. Bessere Kenntnis der Krankheitskonzepte lässt medizinisches Personal auf einen erleichterten kommunikativen Zugang zu Menschen mit Diabetes hoffen, um letztlich deren Eigeninitiative zu stärken und damit (eigene) Behandlungserfolge zu mehren.

Krankheitskonzepte werden von Kultur und Gesellschaft sowie eigenen Werten und Erfahrungen beeinflusst (Linden et al. 1988). Krankheitskonzepte von Behandelnden und Kranken unterscheiden sich und bieten so Konfliktpotential, insbesondere da sie stark emotional geprägt und schwerlich durch Argumente zu ändern sind. Sie dienen als Rechtfertigung für Entscheidungen (Kleinman 1988; Lawton et al. 2008) und enthalten oft unterschiedliche Zielvorstellungen. Für Menschen mit Diabetes scheint der Wunsch nach Kontrolle über die Krankheit bzw. einer „Balance“ im Leben mit ihr ein bestimmendes Motiv zu sein (Gillibrand and Flynn 2001; Paterson and Thorne 2000; Kneck).

Eine Richtung der Forschung zu Krankheitskonzepten sieht die Bewältigung der Krankheit, also die Anpassung an die Krankheit im Alltag und die Identifikation mit ihr als entscheidenden Faktor für Erfolg oder Misserfolg der Behandlung (Kneck; Hörns- 
ten et al. 2004). Bewältigung von Krankheit wird häufig als Ablauf von Phasen charakterisiert, durch die die Erkrankten in Richtung eines Meisterns der Krankheit gehen müssen bzw. wollen (Moser et al. 2008). Es werden sogar Vermutungen über Parallelen zu den Stadien der Bewältigung von Tod und Sterben nach Kübler-Ross angestellt, die ein Mensch durchlaufen muss, bis er die Diagnose Diabetes Typ 2 akzeptiert und dann aktiv am Krankheitsmanagement teilnehmen kann (Kogan 2009).

Ein anderer Aspekt in der Forschung ist die Arzt-Patient-Beziehung. Neuerdings richten sich die Erwartungen von medizinischen Personal und Gesellschaft an Menschen mit Diabetes auf Selbstmanagement und Selbsthilfe (Collins et al. 2009). Die Eigenverantwortung von Menschen mit Diabetes wird beworben. Betroffene sollen Expertise über ihre Krankheit und deren Behandlung erwerben. Gleichzeitig wird die Expertenmeinung des medizinischen Personals mit Bezug auf die evidenzbasierte Medizin als die richtige bestätigt. Holmström und Rosenqvist (2005) unternahmen z.B. den Versuch Krankheitskonzepte zu erforschen - mit dem Ziel, durch Reflexion der Krankheitstheorien die „Missverständnisse“ der Menschen über Diabetes aufzulösen.

Auch wenn also als Ziel betont wird, den Erkrankten - aufgrund ihrer durch Erfahrung erworbenen Expertise - Verantwortung für ihre Behandlung zuzusprechen, wird immer wieder sichtbar, wie schwer es medizinischem Personal fällt, Erkrankte nicht als erziehungsbedürttige Laien zu sehen, sondern in ihrer Mündigkeit anzuerkennen (Paterson 2001). In der Praxis scheint (verständlicherweise) ein heimliches Ideal eine Arzt-Patient-Beziehung zu sein, bei denen Erkrankte ärztlichen Empfehlungen folgen, sie sich dafür aber gemeinsam mit dem medizinischen Personal entschieden haben. Während eine akut kranke Person nur marginal von ärztlichen Therapievorschlägen abweichen wird, also einen gebrochenen Arm gegebenenfalls eine Woche früher belastet als empfohlen, ist es schwer, dieses Konzept von Gesundheit und Krankheit bei chronischen Krankheiten „durchzusetzen“.

Krankheitserfahrungen wurden bisher aus der Perspektive des medizinischen Personals untersucht. Dabei liegt nahe, dass Kranke und Behandelnde - allein aufgrund der Erfahrung - das Konzept Krankheit völlig unterschiedlich begreifen. Natürlich hat es eine innere Logik und ist unerlässlich, dass Ärztinnen und Ärzte kategorisieren, dass sie naturwissenschaftliche Kenntnisse über die Funktion des Körpers als Handlungsgrundlage nehmen und eine Krankheit aus einer gewissen Distanz betrachten. Die 
Kategorisierung in gute und schlechte Kranke - eine gängige Praxis, die sich oft auch direkt oder indirekt in Studien widerspiegelt - verfehlt das Problem oder erzeugt sogar zusätzliche Belastungen in der Therapiebeziehung.

Nicht außer Acht zu lassen ist außerdem, dass Menschen mit Diabetes auch bei „optimalem" Verhalten (und vollständiger Integration der Krankheit in die Persönlichkeit) mit einem Fortschreiten der Krankheit rechnen müssen (Parry et al. 2005). Gleichzeitig wird „Kontrolle“ bei Diabetes Typ 2 aber gesellschaftlich auf eine Weise verhandelt, in der „außer Kontrolle sein“ moralisches Versagen bedeutet (Broom und Whittaker 2004). Der Einfluss von gesellschaftliche Normen und Erwartungen auf die Krankheitserfahrung ist stark (Broom and Whittacker 2004) und spiegelt sich auch in den zum Teil unterschiedlichen geschlechtsspezifischen Erfahrungen wider (Broom and Lenagh-Maguire 2010).

Angesichts der Vielfalt an Verbesserungsvorschlägen für die Diabetesversorgung gibt es vergleichsweise wenig Forschungsarbeiten, welche die Krankheitserfahrung - und nicht das krankheitsbezogene Verhalten - in den Mittelpunkt stellen. Ziel vieler Forschungsgruppen war es, herauszufinden, welches Krankheitsverständnis am besten zu ihrem Lösungsvorschlag der Versorgungsproblematik passt, sei es zur Adherence oder dem Ideal der autonomen, sich mit ihrer Krankheit identifizierenden, selbstständigen Menschen. Das Verständnis von Krankheit kann schwerlich verstanden werden, wenn man durch die Suche nach passenden Beiträgen für bekannte Modelle Nuancen der Krankheitserfahrung verwischt. Während das Versagen der Therapiekonzepte häufig aus mangelndem Verständnis und mangelnder Motivation vonseiten der Erkrankten erklärt wird, wäre es wichtiger, nach möglichen Gründen zu fragen, warum sie die Krankheit nicht oder anders verstehen. Sinnvoll wäre daher, sich von den vorhandenen Modellen und „Idealpatienten“ zu lösen und einen offenen empirischen Bezug zu Denken und Erleben von Menschen mit Diabetes Typ 2 zu suchen. 


\section{Fragestellung und Ziel}

Thema der vorliegenden Arbeit sind Krankheitserfahrungen von Menschen mit Diabetes Typ 2. Theoretischen Überlegungen zu Krankheitskonzepten entsprechend sollte ein gleichermaßen wissenschaftlich überprüfbarer und empathischer Zugang zur subjektiven Seite von Krankheit eröffnet werden. Dafür hatte es sich als sinnvoll erwiesen, die Analyse von vorliegenden Krankheitserzählungen zunächst ohne vorab definierte Fragestellungen, vor allem ohne Vorstellungen von „richtigen“ oder „falschen“ Krankheitskonzepten zu beginnen. Stattdessen sollten im „Inneren“ dieser Erzählungen nach wichtigen lebensweltlichen Phänomenen gesucht und diese interpretiert werden.

Orientiert an der Methode der Grounded Theory, kam es dann im Laufe der Arbeit zu einer Spezifizierung der Fragestellung. Es geht in einem sehr weitgefassten Sinn um „Verzicht" und seinen Gegenpart "Maßlosigkeit": als grundlegende Erfahrung bei der Therapie, als zentrales Thema in der Arzt-Patient-Kommunikation, als Bewertungsmaßstab in der familiären und sozialen Umgebung und schließlich als eigene, oft höchst ambivalente Orientierung.

"Verzicht" erwies sich als überspannende Kategorie zur Untersuchung der Krankheitserfahrungen bei Diabetes Typ 2. Die Rolle von „Verzicht" soll in ihren Eigenschaften und Ausprägungen innerhalb der der Krankheitserfahrung fallübergreifend untersucht werden. Diese Fokussierung - so das Ziel der Arbeit - soll ein für Menschen mit Diabetes spezifisches Kernproblem zum Ausdruck bringen. Aus ihrer subjektiven Perspektive sollen Deutungsmuster der Alltagswelt und Umgangsformen mit dieser chronischen Krankheit nachgezeichnet werden, anstatt diese aus der medizinischen Sicht heraus als hilfreich oder hinderlich in Hinblick auf die therapeutischen Ziele (z.B. metabolischen Zielwerte) zu klassifizieren. 


\section{Methode}

Die Entscheidung für eine „Interpretationsarbeit“ hängt eng mit dem Verständnis des Forschungsgegenstands und der Zielsetzung der Arbeit zusammen. In diesem Kapitel möchte ich zunächst die theoretischen Grundlagen ausführen (4.1), auf denen meine Analyse beruht. Dabei sollen insbesondere zentrale Aspekte der Grounded Theory erläutert werden (4.2). Anschließend charakterisiere ich das von mir verwendete Material und stelle den Kontext dar, in dem es erhoben wurde (4.3). Danach folgt eine Darstellung meines konkreten Vorgehens bei der Auswertung der Interviews (4.4).

\subsection{Theoretische Grundlagen}

\subsubsection{Erforschung von Lebenswelten}

Krankheitskonzepte von Menschen mit Diabetes Typ 2 sind Thema dieser Arbeit (vgl. Kapitel 3), also Erklärungsmodelle und „Deutungen“ von Menschen hinsichtlich ihrer Krankheit (Linden et al. 1988). Diese Modelle sind subjektiv und dynamisch. Sie werden durch Erfahrungen verändert und haben wiederum Einfluss auf das alltägliche Handeln (vgl. Kapitel 2.5). Krankheitskonzepte sind Interpretationen von subjektiven Erlebnissen mit einer Krankheit und damit Versuche, diese zu verstehen. Durch dieses Verstehen wird auch (sinnvolles) Handeln erst möglich. Uns wiederum eröffnen sich die Krankheitserfahrungen anderer ein Stück weit dadurch, dass wir z.B. nach ihrem (Alltags-)Erleben fragen und versuchen, es zu verstehen.

So empfiehlt es sich, den Forschungsgegenstand aus der Perspektive der phänomenologischen Soziologie und des subjektiven Interaktionismus zu betrachten, deren zentrale Thesen ich zumindest andeute. Die phänomenologische Soziologie, als deren Begründer Alfred Schütz gilt, beschäftigt sich mit der Erforschung der Lebenswelt (Fischer 2011, S. 31). Sie wird als subjektive Wirklichkeit der Menschen verstanden, welche diese im Alltag allerdings als "selbstverständlich“ und geltend wahrnehmen (vgl. Honer 2011, S. 112). Schütz entwickelte die Annahme, dass es sich bei sozialwissenschaftlicher Forschung, um „Konstruktionen zweiten Grades handle“, da sie „Konstruktionen der alltäglichen Wirklichkeit“ zum Gegenstand habe, mithilfe derer „sie [die Menschen] diese Welt im Voraus gegliedert und interpretiert [haben]“ (Schütz 1971, S. 67). Die Grundannahmen des subjektiven Interaktionismus nach Herbert Blumer lassen 
sich wie folgt zusammenfassen: In der Interaktion mit anderen Menschen erhalten alle wahrnehmbaren Objekte (ein Baum, ein Haus, aber auch ein Ereignis, eine Meinung, eine andere Person) für Menschen Bedeutungen, auf deren Grundlage sie erst in der Lage sind, zu handeln. Jede Handlung stellt einen neuen „Interpretationsprozess“ dar, so dass sich die Bedeutungen der „Dinge“ (physische Gegenstände, Personen, Handlungen, Gefühle, Leitbilder etc.) fortwährend verändern können (Blumer 1981, S. 84).

Entsprechend dieser Modelle sind menschliche Lebenswelten und damit auch Krankheitskonzepte zu komplex, als dass sie mithilfe von durch theoretische Vorannahmen entwickelte Hypothesen und deren experimenteller Überprüfung erklärbar sein könnten (vgl. Breuer 2009, S. 42-47). Die alleinige Zuordnung von Erfahrungen zu allgemein bewiesenen Sätzen im Sinne einer Subsumption kann der mehrdeutigen und emotionalen Alltagskonzeption von Menschen nicht gerecht werden (ebd.). Da es sich bei den zu erforschenden Konzepten um Interpretationen handelt, liegt es nahe, ein interpretatives Verfahren zu wählen.

\subsubsection{Qualitative Sozialforschung}

Es wird in der Analyse darum gehen, Bedeutungen zu erkennen, die Menschen Objekten zuordnen. Daher möchte ich Deutungsschemata, „Interpretationen der Handelnden“, in den Daten aufdecken und rekonstruieren (Meuser 2011, S. 93). Es die „interpretative, hermeneutische Herangehensweise an das Datenmaterial“ 3 , welche die qualitativen Methoden charakterisiert (Marx und Wollny 2009, S. 106). Im Folgenden sollen zwei entscheidende Aspekte dieses Vorgehens erläutert werden: das Prinzip der Offenheit und die Frage nach der Gültigkeit von Interpretationen.

\section{Prinzip der Offenheit}

Im Gegensatz zu quantitativen Methoden, bei denen vor der Erhebung der Daten konkrete Hypothesen aufgestellt werden, die getestet werden sollen, müssen Ausgangspunkt und Fragestellung zumindest zu Beginn breit angelegt bzw. formuliert sein (Breuer 2009; Marx und Wollny 2009). Eigentlich soll nur das Forschungsfeld ausgewählt, d.h. die Frage beantwortet werden, welchem Teilbereich der alltäglichen Le-

\footnotetext{
${ }^{3}$ Hermeneutik bezeichnet die Wissenschaft des „verstehenden Umgangs mit Texten“, innerhalb der qualitativen Sozialforschung allerdings auch mit „Äußerungen bzw. Interaktionen aller Art unter Einschluss von Alltagskommunikation und eigens erhobenen qualitativen Daten" (Koller 2011, S. 83-84)
} 
benswelt oder welcher Subgruppe der Gesellschaft sich eine qualitative Arbeit zuwendet, (Strauss und Corbin 1996), in dem hier vorliegenden Fall Menschen mit Diabetes Typ 2. Allerdings sind Vorannahmen über den beforschten Lebensbereich nicht zu vermeiden und müssen reflektiert werden (Strauss und Corbin 1996; Breuer 2009). Um Bedeutungen zu verstehen, die Menschen Dingen in einem bestimmten Kontext geben, ist methodisch eine gewisse „Verfremdung“ nötig, ein „Anzweifeln des Gewohnten“, des eigenen Vorverständnisses (Breuer 2009, S. 28).

\section{Gültigkeit der Interpretation}

Durch das Ziel, einen Teil der gedanklich konstruierten Lebenswelt von Menschen mit Diabetes Typ 2, wissenschaftlich zu verstehen, fehlt der Untersuchung die Beweislogik wissenschaftlich erklärender Ansätze (vgl. Breuer 2009, S. 42-47). Ich werde in der Analyse eines Interviews, in dem eine Person ihre Gedankenwelt schildert, nicht die wahre und vollständige, auch nicht die zweifellos richtige Bedeutung wiedergeben können, die sie für das Individuum hat:

„Das Symbolobjekt, dem eine bestimmte Bedeutung verliehen wird, bewahrt stets einen Überschuss an Bedeutungen, es ist - perspektivenbedingt - auch anders lesbar. Verstehens- und Deutungsprozesse bleiben dauerhaft vorläufig und revidierbar." (Breuer 2009, S. 46)

Die lückenlose Aufdeckung des Krankheitskonzepts einzelner Personen soll auch nicht das Ziel der Analyse sein. Vielmehr sollen fallübergreifend aus den Erfahrungen von Menschen mit Diabetes spezifische Muster der möglichen Bedeutungen modelliert/rekonstruiert werden, um sie uns (z.B. als medizinisches Personal, Angehörige, Mitmenschen) verständlicher zu machen.

\subsection{Grounded Theory}

Als Orientierung wurde ein Vorgehen nach den Prinzipien der Grounded Theory ausgewählt. Dieser Forschungsansatz wurde 1967 von Glaser und Strauss entworfen (Glaser und Strauss 1967) und ist "keine spezifische Methode oder Technik“ sondern „vielmehr als ein Stil zu verstehen, nach dem man Daten qualitativ analysiert" (Strauss 1998, S. 30). Strauss formulierte mehrere Hauptelemente, von denen er im Interview mit Legewie und Schervier-Legewie drei als Voraussetzung für das Vorliegen einer Studie nach der Grounded Theory bezeichnete und die im Folgenden näher beleuchtet 
werden (Kap. 4.2.1 bis 4.2.3): Kodieren, Vergleiche und Theoretisches Sampling (Legewie und Schervier-Legewie 2004, Abs. 59; s. a. Breuer 2009, S. 41; s. a. Strauss 1998, S. 51).

\subsubsection{Kodieren}

Unerlässlich für die Analyse ist das Kodieren. Dabei geht es um das Benennen verschiedener „konkrete[r] Daten wie Verhaltensweisen und Ereignisse“, die in Interviews oder ähnlichem Material gefunden werden und die „Indikatoren“ für Konzepte sind (Strauss 1998). Dazu wird während der Betrachtung der Daten die Frage gestellt: „Was geschieht hier eigentlich in den Daten? [...] Was ist hier die eigentliche Geschichte und warum?" (Strauss 1998). Die kreative Erarbeitung von Begriffen, die in den Daten vorkommende allgemeine Konzepte benennen und damit eine gewisse Abstraktion darstellen, ist nötig, um diese analysieren und auf theoretischer Ebene diskutieren zu können (Strauss und Corbin 1996, S. 45). Dennoch sollen Kodenamen eine gewisse „Spezifität“ bezüglich der Daten aufweisen, also keine hohlen, lebensfernen Begriffe sein.

„Es geht um das Entwickeln abstrakter Begriffe, die jedoch noch die Aura ihres empirischen Referenzobjekts besitzen, die gewissermaßen nach ihrer Gegenstandsherkunft riechen und schmecken." (Breuer 2009, S. 76; Hervorhebung im Original)

So wird auch der Begriff Grounded Theory nachvollziehbar: Die Erkenntnisse sind zwar in „theoretischen“ Begriffen formuliert, aber im Gegenstand „gegründet“. Es gibt verschiedene Kodier-Arten (offenes, axiales und selektives Kodieren), auf die ich bei der Beschreibung des konkreten Vorgehens später noch eingehen werde (s. Kap. 4.4).

\subsubsection{Vergleiche}

Das Vergleichen verschiedener Indikatoren, d.h. konkreter Ereignisse in den Daten und vorläufig gebildeter Konzepte ist ein weiteres Grundprinzip, das Voraussetzung für ein Vorgehen nach der Grounded Theory ist. Die Methode des Kontinuierlichen Vergleichens, auch Constant Comparative Method, spielt in allen Phasen der Analyse eine entscheidende Rolle (Charmaz 2006, S. 1874, s.a. Glaser und Strauss 1967; Strauss und Corbin 1996). Verglichen werden Daten untereinander, z.B. Erlebnisse, die in verschiedenen Interviews beschrieben werden. Dabei wird die Aufmerksamkeit

\footnotetext{
${ }^{4}$ "[The] Constant comparative method [is] a method of analysis that generates successively more abstract concepts and theories through inductive processes of comparing data with data, data with category, category with category and category with concept. Comparisons then constitute each stage of analytic development" (Charmaz 2006, S. 187; Hervorhebung im Original)
} 
„Ähnlichkeiten, Unterschieden“ gewidmet und „Klassen“ gebildet: Kategorien (Strauss 1998). Darüber hinaus werden neue Daten immer mit bereits entwickelten Konzepten verglichen und so theoretische Überlegungen geprüft und weiter ausgearbeitet. Durch kontinuierliches Vergleichen lassen sich Konzepte genauer voneinander abgrenzen und definieren. Kategorien werden immer weiter entwickelt und ihre Eigenschaften durch konkrete Beispiele aus den Daten erarbeitet.

Vergleiche sind also die Schnittstelle zwischen Daten und theoretischen Überlegungen. Durch Vergleiche werden (fallübergreifende) Konzepte in verschiedenen Kontexten erst erkannt und unter Berücksichtigung der verschiedenen Kontextfaktoren verstehbar.

An dieser Stelle wird es als sinnvoll erachtet, auf die Begriffe der Induktion und Deduktion einzugehen und ihre Rolle in der Grounded Theory zu erklären. Durch Induktion wird von etwas Speziellem, z.B. einem in den Daten geschilderten Ereignis, auf etwas Allgemeines (ein Konzept, ein Phänomen) geschlossen. Das Spezielle in den Daten ist ein Indikator für ein grundlegendes Phänomen, welches sich in diesem speziellen Kontext in einer bestimmten Variation darstellt. Konzepte werden also zunächst durch induktives Vorgehen entworfen. Diese theoretischen Überlegungen werden im Folgenden deduktiv auf die Daten bezogen und es wird geprüft, ob sich die Daten unter die bereits entwickelte Konzepte subsumieren lassen. Ist dies nicht der Fall, werden neue Konzepte entworfen, vorhandene angepasst bzw. erweitert. Diese „Kombination aus induktivem und deduktivem Denken" ist die Voraussetzung für eine feste Verankerung der theoretischen Überlegungen in den Daten (Strauss 1998; Strauss und Corbin 1996; Strauss und Corbin 1996, S. 107).

\footnotetext{
„Auf der Basis von Erfahrungsdaten aus alltagsweltlichen Kontexten werden - von einer vorläufigen Problematisierungsperspektive ausgehend - theoretische Konzepte und Modellierungen entwickelt und dabei fortwährend rekursiv an die Erfahrungsebene zurückgebunden. Die entsprechende Theorie eines sozialen Weltausschnittes bzw. eines Problemausschnittes wird gegenstandsbegründet herausgearbeitet (,grounded“).“ (Breuer 2009, S. 39)
}

Der analytische Prozess beruht auf immer wiederkehrenden Elementen der Theoriebildung und Theorieprüfung, so dass auf ein wachsendes Vorverständnis ein immer dichteres Verständnis der Daten aufbaut. 


\subsubsection{Theoretisches Sampling und theoretische Sättigung}

Entscheidend ist, „schon nach dem ersten Interview mit der Auswertung zu beginnen, Memos [gemeint sind ausformulierte theoretische Überlegungen] zu schreiben und Hypothesen zu formulieren, die dann die Auswahl der nächsten Interviewpartner nahe legen“ (Legewie und Schervier-Legewie 2004, Abs. 59). In diesem einfachen Satz ist das Vorgehen des Theoretischen Samplings genau charakterisiert: Die Ergebnisse der parallel zur Erhebung verlaufenden Analyse bestimmen, welchen Daten sich die Forschenden als nächstes zuwenden. Ziel ist nicht die Abbildung der genauen Verteilung demographischer Merkmale innerhalb der untersuchten Grundgesamtheit. Die zu untersuchende Gruppe lässt sich demnach nicht wie in der quantitativen Forschung vorab definieren, sondern sie wächst bis zur Theoretischen Sättigung. Corbin formuliert dazu:

„Mit fortgesetzter Analyse emergieren mehr und mehr Konzepte und Kategorien, bis schließlich der/die Forschende das Gefühl hat, die Analyse sei irgendwie saturiert, d.h. dass keine neuen Ideen mehr aus den Daten entstehen." (Corbin 2011, S. 74)

Die eben genannten Vergleiche sind "Motor" der Datenauswahl mit dem Ziel, ein gut durchdachtes und in den Daten begründetes Verständnis über einen Themenbereich zu erlangen:

„Diese sorgfältige Zusammenstellung von Fällen (Sampling) entlang der Dimensionen von Konzepten, wobei deren Ähnlichkeiten und Unterschiede untersucht werden, ermöglicht es den Forschenden, theoretische Erklärungen anzubieten die mehr als eindimensional sind. Je mehr Variationen durch das theoretische Sampling inkorporiert werden, desto variantenreicher ist die Erklärung und desto umfassender die Theorie." (Corbin 2011, S. 74)

\subsection{Kontext und Material}

Das Ausgangsmaterial der vorliegenden Analyse besteht aus narrativen Interviews, welche zwischen 2009 und 2011 für das Website-Projekt „krankheitserfahrungen.de“ erhoben wurden.

\subsection{1 „Krankheitserfahrungen.de“}

„Krankheitserfahrungen.de“ ist eine nach dem Vorbild der britischen Website „healthtalkonline.org“ entwickelte Website der Universitäten Göttingen und Freiburg. „Healthtalkonline.org" wurde mit dem Ziel entworfen, die Erfahrungen kranker Menschen der Öffentlichkeit zugänglich zu machen. Hintergrund war der Eindruck, dass diese sub- 
jektiven Informationen „aus erster Hand“ (Herxheimer et al. 2000) Menschen im Umgang mit der gleichen Krankheit helfen könnten. Ferner soll die Website Angehörigen, Bekannten und anderen Interessierten sowie Menschen, die im Gesundheitssystem tätig sind, helfen, zu verstehen, was für Menschen mit einer bestimmten Krankheit wichtig ist. Zur wissenschaftlichen Entwicklung der Website richtete die britische Forschergruppe die „Database of Individual Patient Experiences (DIPEx)“ ein. Mittlerweile finden sich dort Daten zu über 100 Krankheiten und Gesundheitsproblemen, auch „Module" genannt. "Krankheitserfahrungen.de" wurde durch die Gesellschaft "DIPEx UK“ lizenziert und war an der Gründung des Dachverbandes „DIPEx International“ beteiligt, unter dem „DIPEx“-Websites in vielen anderen Ländern entwickelt worden sind (Arbeitsgruppe krankheitserfahrungen.de 2013).

„Krankheitserfahrungen.de" hat genau wie das britische Vorbild das Ziel, Einblicke in die Erfahrungen von Kranken zu bieten. Bei Besuch der Website besteht die Möglichkeit, unter verschiedenen Modulen eine Krankheit herauszugreifen und dazu originale Video-, Audio- oder Textausschnitte der narrativen Interviews anzuwählen. Es lassen sich gezielt einzelne Personen oder Themen aussuchen. Von großer Bedeutung ist, dass das Anliegen bei der Gestaltung die Darstellung des gesamten Spektrums möglicher Erfahrungen mit einer Krankheit war. Es sollten also nicht nur Erfolgserlebnisse dargestellt werden, wie es auf manchen kommerziell beeinflussten Websites zu Werbezwecken geschieht. Außerdem sollte auch keine "moralische Zensur" stattfinden, d.h. auch Erfahrungen und Handlungen, die vielleicht der "medizinischen“ Sichtweise der Forschenden widersprachen, sollten gezeigt werden.

\subsubsection{Charakterisierung des Materials}

Grundlage der vorliegenden Arbeit ist die Analyse einer Stichprobe aus den 35 narrativen Interviews, welche für die Erstellung des Moduls "Diabetes Typ 2“ auf der Website „krankheitserfahrungen.de“ geführt wurden. Ich selbst war nicht an der Erhebung beteiligt. Um auf der Website Einblicke in Krankheitserfahrungen gewähren zu können, wurden bei der Datenerhebung und der analytischen Aufbereitung für die Präsentation im Internet qualitative Methoden angewendet. Wie bereits erwähnt, sollte auf der Website eine möglichst große Bandbreite von möglichen Erfahrungen mit Diabetes Typ 2 dargestellt werden. 
Für die Interviews wurden Personen daher mit dem Ziel eines Maximum Variation Samples 5 ausgewählt. Schon nach der Erhebung der ersten Interviews wurde mit der Auswertung begonnen. Die Daten wurden hinsichtlich der Themen, die im Zusammenhang mit der Krankheit von Bedeutung sein könnten, exploriert, z.B. Unterzuckerung oder Diabetes im Berufsleben. Daraus wurden theoretische Überlegungen abgeleitet, in welchem Kontext die Erfahrungen sich von den bereits beobachteten Erfahrungen unterscheiden könnten, z.B. in einem anderen Beruf. Aus diesen Hypothesen wiederum wurden Ideen gewonnen, wie und wo weitere Menschen für Interviews rekrutiert werden könnten. Eine Zwischenauswertung und Diskussion in einer Expertenrunde („Advisory Board“) diente demselben Zweck. Dieses Sampling entspricht insofern der oben erläuterten Samplingstrategie, als dass die Auswahl der Interviewten und der später präsentierten Interviewausschnitte ziel- und in gewisser Weise analysegeleitet ist. Im Unterschied zu diesem Verfahren war allerdings das Ziel der Untersuchung entsprechend der „Forschungsfrage“ bei „krankheitserfahrungen.de“ von vornherein auf das Explorieren und Präsentieren von möglichst vielen Themen beschränkt.

In der vorliegenden Analyse wurde statt eines solchen Purposeful Samplings („zielgerichtetes" Sampling) mit dem Ziel eines Maximum Variation Samples die Methode des Theoretical Sampling angewendet (vgl. Coyne 1997) mit dem Ziel, die Analyse durch datengegründete theoretische Überlegungen „zuzuspitzen“ (vgl. Kap. 3 und Ergebnisse). Das Sampling führt dann zu einer möglichst tiefgehenden Ausarbeitung einiger weniger, zu Beginn der Studie noch nicht feststehender Kategorien, während das für das Websiteprojekt verwendete Sampling eine eher horizontale, überblickende Darstellung relevanter Themen mit Beispielen produziert. Ergebnis der qualitativen Textanalyse der 35 Interviews zu Diabetes Typ 2 durch die Göttinger Forschungsgruppe war die Identifikation von Themenkomplexen, wie z.B. „Diagnosestellung und Symptome" und Veränderung der Lebensgewohnheiten mit Unterthemen wie z.B. "Mehr Bewegung“, „Weniger Pfunde“. Diesen wurden dann zusammenfassende Texte sowie beispielhafte Interviewausschnitte zugeordnet. Diese Ergebnisse führten wie

\footnotetext{
${ }^{5}$ Auf der Website „www.krankheitserfahrungen.de“ wird das Vorgehen bei der Stichprobenwahl wie folgt beschrieben: „Ziel der Studie ist es, möglichst viele unterschiedliche Erfahrungen, Ursachen, Therapiemethoden und Lebensweisen mit dem Problem aufzunehmen, damit die Website ein entsprechend breites Spektrum anbieten kann. Dazu werden Interviews mit Personen aller betroffenen Altersstufen, mit den unterschiedlichsten Lebenssituationen und an Orten über ganz Deutschland verteilt geführt. Dies führt zu einem sogenannten "maximum variation sample", einer größtmöglichen Streuung aller Erfahrungen, um zu gewährleisten, dass alle wichtigen Aspekte in der Studie auftauchen“ (Arbeitsgruppe krankheitserfahrungen.de 2013).
} 
gewünscht zu einer durch qualitative Methoden fundierten Darstellung der Bandbreite an Diabeteserfahrungen auf „krankheitserfahrungen.de“.

\subsubsection{Narrative Interviews}

Bei dem für diese Analyse zur Verfügung stehenden Datenkorpus handelt es sich um sogenannte Narrative Interviews. Die Methode geht auf Fritz Schütze zurück (Przyborski und Wohlrab-Sahr 2008, S. 92). Über einen Erzählstimulus, eine „eindeutig narrative Ausgangsfrage“, soll „eine Stehgreiferzählung über eigene Erfahrungen“ entstehen (Riemann 2011, S. 121-122), möglichst „ohne Unterbrechungen durch den Forscher“ (ebd.). Die erzählende Person wird bestimmte, für sie persönlich relevante Dinge hervorheben, andere verkürzen oder auslassen, gewichtet die Ereignisse also ganz persönlich. So entsteht ein datenreiches Material, in dem die beforschte Person selbst das Relevanzsystem festlegt und dieses „Vorrang“ hat "gegenüber jenem der Interviewerinnen“ (Przyrborski und Wohlrab-Sahr 2008, S.87; Hervorhebung im Original). Nachfragen dürfen erst nach Beendigung der Eingangserzählung der interviewten Person gestellt werden. An dieser Stelle möchte ich die Eingangsfrage zitieren, die in den Interviews für das Diabetesmodul bei „krankheitserfahrungen.de“ als Eingangsstimulus verwendet wurde ${ }^{6}$ :

Sie wissen, wir interessieren uns für die Erfahrungen von Diabetes-Patienten mit der Erkrankung. Ich möchte Sie nun bitten, mir von Ihren Erfahrungen zu erzählen, vielleicht von dem Zeitpunkt an, als Sie das erste Mal das Gefühl hatten, dass irgendetwas nicht in Ordnung ist, oder als der Diabetes bei Ihnen festgestellt wurde. Bitte erzählen Sie über alles, was dann passiert ist bis heute. Sie können sich für das Erzählen so viel Zeit nehmen, wie Sie möchten. Ich werde Sie erst einmal nicht unterbrechen, sondern mir nur Notizen machen, auf die ich dann später Stück für Stück zurückkomme.

\subsection{Praktische Herangehensweise}

\subsubsection{Datenauswahl}

Aufgrund der sehr großen Datenmenge des Ausgangsmaterials (35 Interviews) wurden zunächst im Sinne eines Überblicks Alter, Krankheitsdauer, Krankheitsverlauf, Familienstand, Tätigkeit und mögliche Besonderheiten der Interviewten in einer Tabelle festgehalten. Zu solchen „Besonderheiten“ zählten z.B. von der interviewten Person erwähntes „Fasten“, „Frustessen“ oder „Gründung einer Selbsthilfegruppe“. Dieses „Bekanntmachen“ mit dem Material sensibilisierte für relevante Aspekte. Auffallend

${ }^{6}$ Die Erhebung der Interviews war nicht Teil der vorliegenden Arbeit. Für die Analyse wurde eine Strichprobe aus der Gesamtheit der für „krankheitserfahrungen.de“ erhobenen Interviews gezogen (s. Kap. 4.4.1). 
war unter anderem, dass Abnehmen ein immer wiederkehrendes Thema in den Interviews darstellte.

Nach dieser ersten Durchsicht wurde ein Interview zur Feinanalyse ausgewählt, vornehmlich nach dem Zufallsprinzip. Person 32 (im Folgenden P32) ist männlich und war zum Zeitpunkt des Interviews 35 Jahre alt. P32 ist im Alter von 24 Jahren an Diabetes erkrankt und wurde zunächst mit Tabletten behandelt. Zur Erreichung erwünschter Blutzuckerwerte wurde er auf eine Insulintherapie umgestellt, schließlich reichten aber wieder Tabletten zur Blutzuckersenkung aus. Subjektiv besonders augenfällig in diesem Interview waren Erzählungen über die Teilnahme an einem Diätprogramm und das Fasten während des Ramadans.

Eine erste Auswertung dieses Interviews führte zu der vorläufigen Hypothese, dass Übergewicht eine große Rolle in der Krankheitserfahrung mit Diabetes Typ 2 spielen könnte. Um diese Hypothese zu prüfen, wurde - ebenfalls nach eher augenscheinlichen Kriterien - das Interview mit P30 (weiblich, 42 Jahre alt, Erkrankungsalter: 36 Jahre) ausgewählt. Auch für sie hatten der ersten Durchsicht zufolge die Themen Übergewicht und Diäten eine große Bedeutung. Ebenfalls wurde P21 (weiblich, 67 Jahre alt, Erkrankungsalter: 58 Jahre) hinzugezogen, die wiederrum erzählte, immer schlank gewesen zu sein.

Deutlich wird, dass bei diesem Vorgehen das Kodieren und Vergleichen der Daten ausschlaggebend für die weitere Datenauswahl war. P30 wurde wegen der Ähnlichkeit bezüglich der Gewichtsproblematik herangezogen und stellt so einen minimalen kontrastiven Vergleich dar. Zum maximalen kontrastiven Vergleich wurde P22 herangezogen, bei der Gewicht keine Rolle zu spielen schien (vgl. Rosenthal 2011, S. 97; vgl. Strübing 2011, S.155). Diese Vorgehensweise ist eine Möglichkeit, Daten nach dem bereits erläuterten Prinzip des Theoretical Sampling der Grounded Theory auszuwählen. Daten wurden sukzessive herangezogen mit dem Ziel, die Entwicklung der Konzepte und damit die der theoretischen Überlegungen voranzutreiben.

Die Auswahl der ersten Interviews P32, P30 und P21, die gerade beispielhaft beschrieben wurde, war gegenüber einem späteren Zeitpunkt noch wenig „in den Daten begründet“. Sie richtete sich auch noch nach oberflächlichen Kriterien und Vorannahmen: dass z.B. Alter oder Geschlecht die Erfahrung prägen würden. Demgegenüber verlief 
das Sampling mit Weiterentwicklung der theoretischen Überlegungen und Fokussierung der Fragestellung (vgl. Kap. 3 und Ergebnisse) immer zielgerichteter. Auch geschah es fallunabhängig: Es wurden nicht mehr ganze Interviews Zeile für Zeile analysiert, sondern einzelne Ereignisse herausgegriffen und genauer mit solchen in anderen Interviews verglichen, um Konzepte zu verdichten. Strauss und Corbin (1996) betonen, dass nicht „Personen an sich“ für das Sampling ausgewählt werden, sondern "Vorkommnisse“ (S. 149). Wenn hier also von einer Stichprobe von 14 Personen die Rede ist, ist damit gemeint, dass Daten aus 14 Interviews herangezogen wurden, bis die Kategorien im Sinne einer Theoretischen Sättigung ausgearbeitet waren (s. Kap. 4.2.3). Die Stichprobe wird im Ergebnisteil beschrieben werden (5.1).

\subsubsection{Kodier-Verfahren}

Klassischerweise werden drei Arten des Kodierens beschrieben, welche im Laufe des Analyseprozesses immer wiederkehrend eingesetzt werden: (1) Offenes Kodieren findet sehr nah am Text statt, z.B. werden Interviews Zeile für Zeile analysiert, und für verschieden große Sinneinheiten - einen Textabsatz, einen Satz, ein Wort - Namen für die dahinter stehende Bedeutung vergeben. Das „Benennen eines Phänomens“ erzeugt eine "fortlaufende Aufmerksamkeit" gegenüber dem Phänomen (Strauss und Corbin 1996, S. 44). Erst die Kodenamen ermöglichen es außerdem, über die Phänomene oder Konzepte zu kommunizieren und Fragen über sie zu stellen. (2) Axiales Kodieren ist nach Strauss und Corbin (1996, S. 75) das In-Beziehung-setzen von Kategorien. (3) Selektives Kodieren (ebd., S. 94) hat zum Ziel, eine alle Kategorien verbindende Kernkategorie zu finden und so die Erkenntnisse über den erforschten Bereich zusammenfassen, als Integration der Forschungsergebnisse:

\footnotetext{
„Am Ende der Theorieentwicklung steht - nach dem Lehrbuch-Idealfall - eine sogenannte "Kernkategorie“, die einen Schlüssel zum Verständnis des fokussierten Problemthemas liefert und das strukturierende Zentralkonzept für die gefundenen Konzepte bzw. für die entwickelte Bereichstheorie herstellt." (Breuer 2009, S. 53)
}

Nun soll das Herangehen an die Daten genauer dargestellt werden. Strauss und Corbin regen dazu an, mithilfe des offenen Kodierens die Daten „aufzubrechen“ (Strauss und Corbin 1996, S. 44). Die ersten Interviews werden Zeile für Zeile kodiert. Textabschnitte werden so bestimmt, dass sie eine Sinneinheit ergeben. Kodenamen werden so ausgewählt, dass sie möglichst spezifisch Handlungen bzw. Ereignisse in den Erfahrungsgeschichten reflektieren und gleichzeitig die Qualität eines Konzepts haben, 
also vergleichbare und abstrakte Ideen ausdrücken, die auf theoretischer Ebene diskutiert werden können. Beispiele sind „Unterstützung vom Partner erhalten“ oder „Verzichten als hart erleben“. Zu Beginn wird bewusst versucht, für die Kodenamen die Verbform zu wählen, um das Phänomen als Handlungsereignis - mit der interviewten Person als individuellem Akteur - in einem bestimmten Kontext, mit bestimmten Motiven und mit bestimmten Konsequenzen kenntlich zu machen. So bleibt der Bezug zu den spezifischen Daten erhalten.

Einige Themen oder Kontexte wurden von den meisten Interviewpartnern immer wieder in kleinen Erzählepisoden aufgegriffen. Diese scheinen von großer Bedeutung zu sein, z.B. das Messen von Werten. Diese Themen sind manchmal sehr offensichtlich in den Daten zu entdecken und werden dann relativ früh im Kodierungsprozess auch im Kodenamen als erstes Wort kenntlich gemacht, z.B. „med. Kontrolle(+ Nachsatz, z.B. sich diskriminiert fühlen)“. Dies bietet den Vorteil, dass Konzepte, die im gleichen Kontext eine Rolle spielten schon gruppiert werden und so einander gegenübergestellt werden können. Andere immer wiederkehrende Themen, sind weniger offensichtlich. So fällt z.B. auf, dass die Beurteilung oder das Bild des eigenen Körpers eine Rolle spielt oder Regeln, die entwickelt wurden, um im Alltag eine Orientierung bei der Ernährung zu haben. Diese „Kodegruppen“, die auch als erste vorläufige Kategorien bezeichnet werden können, werden dann mit den Begriffen „Körperbild“ und „Regeln“ überschrieben.

In Aussagen von zwei Interviewten wurde das Vorhandensein eines Rückzugsortes als bedeutsam für die Befragten wahrgenommen und mit dem Begriff „Eigener Raum“ kodiert. Für eine interviewte Person wird dieser „Raum“ durch Sport repräsentiert, für eine andere durch das morgendliche Trinken einer Tasse Tee, wenn die restlichen Familienmitglieder das Haus bereits verlassen haben. Indem sowohl die Schilderung der Bedeutung von Sport als auch die des Zeitungslesens unter dem abstrakteren Konzept „Eigener Raum" zusammengefasst werden, lassen sich diese mit anderen in Beziehung setzen und vergleichen, z.B. mit dem Konzept „Unterstützung vom Partner erhalten“. Beide Konzepte sind mit ganz unterschiedlichen Ausprägungen und Alltagserlebnissen „gefüllt", also in den Daten „begründet“ (grounded). So haben wir die Erkenntnis gewonnen, dass ein Rückzugsort für Menschen mit Diabetes von Bedeutung 
sein kann. Wir können uns auch dessen unterschiedliche Eigenschaften und Ausprägungen vorstellen.

\subsubsection{Ausbildung und Nutzung theoretischer Sensibilität}

Theoretische Sensibilität gegenüber dem Forschungsgegenstand ist eine unumgängliche Fähigkeit, die sich die Person, die im Sinne der Grounded Theory arbeiten möchte, aneignen muss. Sie erfordert ein Gefüh/ und Offenheit für das Spektrum von Bedeutungen, die der entsprechende Themenbereich für Menschen haben kann. Man sollte sich von dem zu erforschenden Lebensbereich „entfremden“, also die eigene professionelle und die Laien-„Brille“ absetzen (vgl. Breuer 2009, S. 59) Ich muss z.B. meine Vorannahmen über das Leben mit Diabetes aus der Perspektive der Medizinstudentin, Verwandten einer Diabetikerin, Forscherin reflektieren bzw. zurückstellen, wenn ich Interviews lese und verstehen will. Meine Vorannahmen bilden, wenn ich sie mir bewusst mache, einen „Anfangs-Fundus“ der theoretischen Sensibilität: ich kenne die Symbol-Bedeutungen, die ich mit Diabetes verbinde, werde aber auch darüber reflektieren, dass die beforschten Individuen Dinge ganz anders verstehen könnten.

Gerade zu Beginn der Analyse ist es nötig, die Theoretische Sensibilität zu erhöhen, um Konzepte entwickeln zu können und Kodenamen dafür zu finden. Sie hilft aber auch, bei der Durchsicht weiterer Daten (z.B. in anderen Interviews) einen fokussierten Blick auf weitere Ausprägungen einer Kategorie zu haben, also immer leichter „hinter die Kulissen“ zu blicken und die Bedeutung hinter einer Aussage wahrzunehmen. Zur „Schärfung“ der Sensibilität gibt es verschiedene Techniken (Strauss und Corbin 1996, S. 56-74). Eine Möglichkeit, die theoretische Sensibilität zu erhöhen, besteht darin, Fragen zu stellen.

Was hat die Person erlebt? - eine Mitteilung der Laborwerte durch den Arzt

Wie hat sie es wahrgenommen und an welchen Worten wird dies deutlich? - die Person beschreibt sich schon vor der Konsultation als nervös und ängstlich, mit einer Vorahnung, dass sie getadelt werden wird.

Welche Person nimmt eine Laborbefundmitteilung wie wahr und warum? Von welchen Faktoren werden die emotionalen Reaktionen beeinflusst? Wie läuft die Befundmitteilung bei anderen Krankheiten ab? Mit welchen anderen Mitteilungen ist sie vergleichbar?

Eine andere Taktik ist die Analyse eines einzelnen Wortes, einer Phrase oder eines Satzes. Dies kann helfen, Begrifflichkeiten zu überdenken, statt von der ersten eigenen Interpretation auszugehen. Dadurch öffnet man sich für die möglichen Bedeutungen, 
die eine interviewte Person damit verbinden könnte. Ich möchte dies an zwei Beispielen erläutern:

Mehrfach kommt in den Interviews der Begriff „Volkskrankheit“ vor. Im ersten Moment assoziiert man damit vielleicht nur „,häufige“ Krankheit. Ein Brainstorming führt dann zu Assoziationen: für die Gesellschaft typisch, Wohlstand, Zivilisationskrankheit, Lebensstil, Übergewicht, kardiovaskuläre Erkrankungen, ökonomische Belastung, (keine) Schuld, nicht ungewöhnlich, „normal“, Alter, Stress, Burnout, Depression, Hilflosigkeit, Chronizität.

Assoziationen zu dem ebenfalls häufig genannten Begriff „Frustessen“ sind: Teufelskreis, Unzufriedenheit, Ärger vergessen, sich gehen lassen, an sich denken, Belohnung, Schuldgefühle, Scham, Essstörung, Bulimie, Binge Eating, ungesunde Nahrungsmittel, Fernsehen, Bewegungsmangel, Einsamkeit, heimlich.

\subsubsection{Atlas.ti}

Beim offenen Kodieren wird die Software Atlas.ti (Atlas.ti $\mathrm{GmbH}$ ) verwendet, eine der vielen Programme für eine Computer Assisted Qualitative Data Analysis (CAQUDAS). Der wesentliche Vorteil von Atlas.ti besteht in der Möglichkeit, immer wieder schnellen Zugriff auf bestimmte Daten innerhalb des großen Korpus zu haben. Alle Transkripte der 35 Interviews, also des vorgefundenen Gesamtsamples waren über die Software gespeichert. Die Interviews konnten einzeln angewählt werden. Ausgewählte Textabschnitte, hinter denen eine Sinneinheit vermutet wurde, konnten markiert und dann leicht und übersichtlich am Rand mit Kodenamen versehen werden (s. Abbildung 1, S. 45). Über den „Code Manager“ ließen sich außerdem alle zu einem oder mehreren "Code“ gehörenden Textabschnitte abrufen. Diese Funktion war hilfreich, um Interviewzitate zu vergleichen und so Konzepte zu verfeinern.

\subsubsection{Memos}

Im Verlauf des Kodier-Prozesses werden, z.B. durch Zusammenfassung von Kodes zu Gruppen, sogenannte Kategorien gefunden, die durch theoretische Überlegungen und die immer wiederkehrende Beschäftigung mit neuen Daten verdichtet werden sollen. Diese Ausarbeitungen werden möglichst genau dokumentiert: in Form von sogenannten Memos (Strauss und Corbin 1996, S. 169). Memos kommt während des ganzen Analyseprozess eine große Bedeutung zu, eigentlich überall dort, wo Vergleiche angestellt werden (vgl. Kap. 4.2.2). Memos stützen die Kodevergabe beim offenen Kodieren. Sie sind auch Grundlage für das axiale und selektive Kodieren, also die Verknüpfung von Konzepten und Kategorien und dokumentieren diese Prozesse. Memos 
sind schriftlich festgehaltene theoretische Überlegungen, die einzelne Wörter ${ }^{7}$, Vergleiche von verschiedenen Zitaten oder Fällen, einzelne Kategorien oder Vergleiche zwischen Kategorien betreffen können. Im Verlauf wurden außerdem Memos zu den Einzelfällen verfasst, die zu Kurzgeschichten („Fall-Memos“) zusammengefasst wurden. Diese halfen dabei, Handlungen und Gedanken der Interviewten fallübergreifend zu vergleichen. Entsprechend der Grounded Theory wurde im Analyseprozess immer wieder zwischen offenem, axialem und selektivem Kodieren gewechselt, also mithilfe von Atlas.ti direkt am Text kodiert und parallel theoretische Gedanken in Form von Memos formuliert.

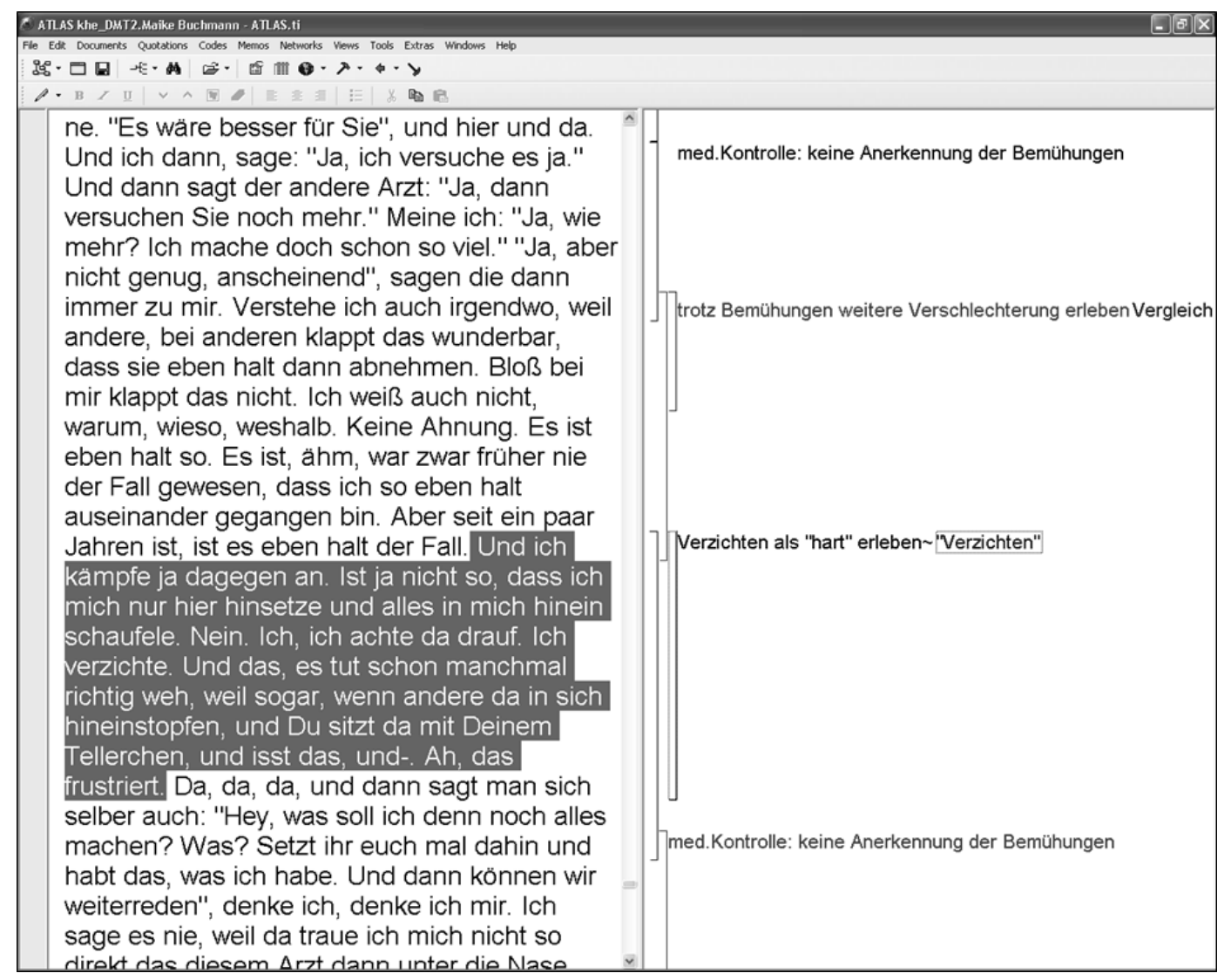

Abbildung 1: Kodieren mit Atlas.ti (Atlas.ti $\mathrm{GmbH})$

\subsection{Datenschutz und Genehmigung durch die Ethik-Kommission}

Die Untersuchung lag der Ethik-Kommission der Universität Göttingen vor und wurde unter der Antragsnummer 18/1/09 bearbeitet und gebilligt. Alle zitierten Interviewausschnitte liegen in anonymisierter Form vor.

\footnotetext{
7 Siehe das auf S. 44 genannte Beispiel eines „Memos“ über das Wort „Volkskrankheit“.
} 


\section{Ergebnisse}

\subsection{Vorbemerkungen zur Kernkategorie und Darstellung der Ergebnisse}

Durch das offene Kodieren in wenigen Interviews zu Beginn der Analyse wurden erste relevante Konzepte entdeckt. Früh wurde augenfällig, dass sich viele Interviewte mit „Körpermaßen“, „Laborwerten“ „fehlender Spürbarkeit von Diabetes“, „medizinischer Kontrolle“, „Verzicht“ und „Regeln“ auseinandersetzten. Zu diesen Konzepten wurden Memos geschrieben, in denen theoretische Überlegungen angestellt wurden. Zu „Körpermaßen“ wurde z.B. folgende Hypothese aufgestellt:

Körpermaße scheinen für viele Interviewte von großer Bedeutung zu sein. Ärztliche Kommentare zu Übergewicht können sie persönlich sehr kränken. Möglicherweise wird Kritik am Übergewicht als Kritik am Lebensstil verstanden, der bei Diabetes Typ 2 immer wieder Verhandlungspunkt in der ärztlichen Behandlung ist.

Diese und ähnliche analytische Ideen leiteten die Auswahl weiterer Fälle, sodass z.B. das Interview einer Person herangezogen wurden, die übergewichtig war und vom Arzt aufgefordert wurde abzunehmen, sowie einer Person, die nicht abnehmen musste. Beim weiteren Kodieren wurden die Hypothesen bedacht und mit den betrachteten Daten verglichen. Die in den Daten identifizierten Ereignisse konnten entweder unter bereits bestehenden Konzepten zu Kategorien zusammengefasst werden, die dann in ihren verschiedenen Eigenschaften und Ausprägungen immer besser verstanden wurden, oder es wurden neue Aspekte der Erfahrung sichtbar. Die Entwicklung der Kategorien in Form von vorläufigen Hypothesen wurde immer wieder am Datenmaterial überprüft.

Nach und nach wurden die Kategorien im Sinne des axialen Kodierens (s. Kapitel 4.4.2) zueinander in Beziehung gesetzt. Erkannt wurde dadurch z.B., dass die Diskrepanz zwischen Messwerten und spürbaren Symptomen dazu führen kann, dass ärztliche Forderungen als verwirrend wahrgenommen werden, z.B. auf bestimmte Lebensmittel zu verzichten, obwohl keine Krankheit spürbar ist. Es wurde außerdem deutlich, dass sowohl Körpermaße als auch Laborwerte als moralische Prüfmarken dienten, nach der der Lebensstil von Menschen mit Diabetes bewertet wird. Diese und ähnliche Hypothesen zur sinnhaften Verknüpfung von Kategorien wurden nicht nur in Memos durchdacht, sondern erneut am Datenmaterial überprüft. Auf allen Ebenen der Analyse wurden die Ergebnisse mit Mitgliedern der Arbeitsgruppe diskutiert. 
Im Sinne des selektiven Kodierens (s. Kap. 4.4.2) wurde eine alle Hauptkategorien verbindende Kernkategorie gesucht. Es zeigte sich, dass „Verzicht" - als Forderung, als Orientierung, als (negatives, sehr wohl auch positives) Erleben, als (eigener und fremder) Bewertungsmaßstab - besonders beherrschend für die Erfahrungen mit Diabetes Typ 2 war.

Im Folgenden sollen die Ergebnisse dargestellt werden, gegliedert mit dem Ziel, die verschiedenen Eigenschaften und Ausprägungen des Phänomens "Verzicht“ zu veranschaulichen. Dargestellt wird eine Art „Umkehr" des Analyseprozesses. Während im Analyseprozess aus Daten theoretische Überlegungen induziert wurden, die dann deduktiv an weiteren Daten überprüft wurden, findet sich bei der Präsentation der Ergebnisse folgender Aufbau: Zunächst werden die Hypothesen genannt, die anschließend mit Auszügen aus den Daten (Zitaten) belegt werden. Die Kernkategorie soll dabei immer weiter „aufgefächert" und im Detail erklärt werden.

\subsection{Beschreibung der Stichprobe}

Der Aufbau der Stichprobe erfolgte, wie im vorrausgehenden Kapitel dargestellt, im Sinne des Theoretical Samplings sukzessive mit Fortschreiten der Analyse. Tabellarisch soll an dieser Stelle ein kurzer Überblick über das Sample geboten werden (Tabelle 1). Es werden Erkrankungsalter, Krankheitsdauer und Medikation der Interviewten aufgeführt, obwohl diese Aspekte, wie in Kap. 4.4.2 beschrieben, nur von geringer Bedeutung für den Einbezug eines Interviews (Falls) in die Stichprobe waren. Weiterhin wird der Versuch unternommen, die Besonderheiten bzw. Charakteristika zu benennen, die zur Fallauswahl führten.

Die Stichprobe setzt sich aus 8 Frauen und 6 Männern zusammen. Der Altersdurchschnitt lag bei 57,6 Jahren, mit einer Spanne von 35 - 73 Jahren. Die Personen hatten die Diagnose Diabetes Typ 2 durchschnittlich 11,9 Jahre vor dem Interviewzeitpunkt erhalten, die Krankheitsdauer lag zwischen 3 und 32 Jahren. Die Mehrzahl (7) hatte seit weniger als 10 Jahren Diabetes, bei 4 Personen betrug die Krankheitsdauer zwischen 10 und 19 Jahren; 3 Personen waren länger als 20 Jahre lang krank. Sieben Personen wurden mit oralen Antidiabetika behandelt, 6 weitere zusätzlich oder ausschließlich mit Insulin, eine Person nahm keine blutzuckersenkenden Medikamente ein. Die Mehrheit der Personen übte zum Zeitpunkt des Interviews einen Beruf aus. 


\section{Fünf Personen waren berentet. Der Großteil war verheiratet (außer einer verwitweten und einer getrennt lebenden Frau) und hatte Kinder.}

\begin{tabular}{|c|c|c|c|c|c|}
\hline $\mathbf{P}^{8}$ & $\mathbf{G}^{9}$ & $A^{10}$ & $\mathrm{DM}^{11}$ & Medikation $^{12}$ & Kurzbeschreibung, Auswahlgrund ${ }^{13}$ \\
\hline P32 & $\mathrm{m}$ & 35 & 11 & $\begin{array}{l}\text { Basal unterstützte } \\
\text { orale Therapie. Vor } \\
\text { Diätprogramm zu- } \\
\text { sätzlich kurzwirksa- } \\
\text { mes Insulin 3x/d. }\end{array}$ & $\begin{array}{l}\text { P32 erzählt, dass er nach der Diagnose zunächst weiter lebte wie zuvor: „Ar- } \\
\text { beiten, [...] Familie, am Wochenende schön Grillen“ (P32:383). }{ }^{14} \text { Der Herzin- } \\
\text { farkt wenige Jahre später war ein Wendepunkt: P32 ließ sich in einer Diabetes- } \\
\text { klinik behandeln. „Intensiv“ war für ihn die Teilnahme an einem Diätprogramm, } \\
\text { die zeitlich mit dem Fastenmonat Ramadan zusammenfiel. Er erzählt, während- } \\
\text { dessen erfolgreich abgenommen und seine Laborwerte verbessert zu haben. }\end{array}$ \\
\hline P30 & w & 42 & 18 & $\begin{array}{l}\text { Orale Therapie. } \\
\text { Evtl. Umstellung auf } \\
\text { Insulintherapie ge- } \\
\text { plant. }\end{array}$ & $\begin{array}{l}\text { P30 ist enttäuscht. Immer wieder wird sie aufgefordert abzunehmen und hat } \\
\text { das Gefühl, ihr werde unterstellt, dass sie „faul“ sei (P30:067), weil ihr Gewicht } \\
\text { nicht weniger wird und sie ungünstige Laborwerte hat. Dabei strengt sie sich so } \\
\text { an: Sport, Abwiegen der Lebensmittel und dazu ihr Job als Hausmeisterin und } \\
\text { im Haushalt. Erschwert wird das Abnehmen durch Kinder und Ehemann, die } \\
\text { „Schmackhaftes“, anstelle von „gesundem“ Essen von ihr erwarten. }\end{array}$ \\
\hline P21 & w & 67 & 8 & Orale Therapie. & $\begin{array}{l}\text { P21 ist von der Diagnose Diabetes „schockiert“, zumal „Kolleginnen und Freun- } \\
\text { dinnen, die beleibter sind“ nicht Diabetes haben - und sie „als schlanke Person“ } \\
\text { schon (P21:622). Sie vermutet erbliche Ursachen. Die von ihrem Hausarzt aus- } \\
\text { gesprochene Empfehlung, sie solle weniger Weißbrot essen und auf Kuchen } \\
\text { verzichten, bezeichnet sie als „hart“ (P21:237) und beschreibt es als schwierig, } \\
\text { auf den Kaffee und Kuchen am Nachmittag zu verzichten. Für ihre guten La- } \\
\text { borwerte wird sie von ihrer Ärztin gelobt. }\end{array}$ \\
\hline P16 & $\mathrm{m}$ & 57 & 3 & $\begin{array}{l}\text { Erst } 3 \text { Monate lang } \\
\text { Insulintherapie, da- } \\
\text { nach orale Thera- } \\
\text { pie. } 9 \text { Monate nach } \\
\text { Diagnose keine } \\
\text { Medikation mehr. }\end{array}$ & $\begin{array}{l}\text { P16 erzählt, dass er bei Diagnose einen so stark erhöhtem Blutzuckerspiegel } \\
\text { gehabt hat, dass er auf der Intensivstation behandelt worden (P16:0186) und } \\
\text { auf Insulin eingestellt worden ist. Im Krankenhaus hat er begonnen, sich streng } \\
\text { an eine Diät zu halten und in den } 9 \text { Monaten nach der Diagnose ungefähr } 40 \\
\text { kg Körpergewicht verloren (P16:2010). Seither muss er keine Diabetes-Medi- } \\
\text { kamente mehr einnehmen. Aus seiner Sicht ist Diabetes „mit Disziplin ganz gut } \\
\text { in [den] Griff zu kriegen“(P16:1738). }\end{array}$ \\
\hline P20 & W & 54 & 7 & Orale Therapie. & $\begin{array}{l}\text { P20 hat die Diagnose Diabetes in dem Jahr erhalten, in dem ihre Mutter gestor- } \\
\text { ben ist und bringt sie mit der psychischen Belastung in Verbindung. Als die } \\
\text { "Kinder [...] klein waren“ hat sie sich und ihren Körper „vergessen“ (P20:053). } \\
\text { Abgenommen hat sie in ihrem eigenen „Tempo“ (P20:041). Wenn sie zum Früh- } \\
\text { schwimmen geht, ist sie „stolz“, weil sie ihrem Körper "etwas Gutes“ tut } \\
\text { (P20:053). Sie probiert immer wieder neue Dinge in der Ernährung aus, z.B. } \\
\text { Senf statt Butter zu verwenden (P20:377). }\end{array}$ \\
\hline
\end{tabular}

Tabelle 1: Stichprobe (wird fortgesetzt)

\footnotetext{
8 Person: Anonymisierte Personenidentifikationsnummer innerhalb des Projekts Krankheitserfahrungen, für das 35 Interviews erhoben wurden, aus denen für diese Analyse eine Stichprobe von 14 Interviews gezogen wurde. Die Personen werden hier in der Reihenfolge genannt, in der sie in das Sample aufgenommen wurden. Deshalb entspricht die Aufzählung nicht der normalen Zahlenabfolge.

${ }_{9}^{9}$ Geschlecht: w- weiblich, m- männlich.

${ }^{10}$ Alter: in Jahren, zum Zeitpunkt der Erhebung.

11 Diabetes mellitus: Dauer der Erkrankung in Jahren, d.h. seit der Diagnose vergangene Jahre bis zum Interviewzeitpunkt.

12 Medikation: Diese Angaben beziehen sich ausschließlich auf Antidiabetika. Sie sind den Interviews entnommen, d.h. der Selbstauskunft der Interviewten. Stellennachweis in den Interviews ist an anderer Stelle dokumentiert. Basal unterstützte orale Therapie bedeutet die Kombination von oralen Antidiabetika mit einem Basalinsulin.

${ }_{13}$ Auswahlgrund: gemeint ist die Aufnahme ins Sample. Beim theoretischen Sampling ist die Auswahl der Interviews analysegeleitet. In dieser Spalte wird in sehr reduzierter der Sampling-Prozess nachgezeichnet.

${ }^{14}$ Die Belege verweisen auf die Interviewstellen, so wie sie in den Transkripten bei Atlas.ti vorlagen.
} 
P22 w $52 \quad 6 \quad$ Insulintherapie.

P1 w 51 $11 \quad \begin{aligned} & \text { Erst 5 Jahre lang } \\ & \text { orale Therapie, } \\ & \text { dann Insulinthera- } \\ & \text { pie. }\end{aligned}$

\begin{tabular}{lllll}
\hline P2 & $\mathrm{m}$ & 64 & 5 & Orale Therapie.
\end{tabular}

P22 „verzichtet nicht auf Kartoffeln oder Nudeln oder Reis“, sondern isst so wie vor der Diagnose, weil sie keinen Unterschied zwischen einer normalen gesunden Ernährung und dem, was sie als Diabetespatientin zu sich nehmen sollte, sieht (P22:066). Sie hat bemerkt, dass statt süßer Lebensmittel eher fettige ihren Blutzucker in die Höhe treiben (P22:70). Sie erzählt, dass ihre Mutter und ihre Großmutter „vollschlank“ waren (P22:138). Sie sagt, sie selbst ist nur um den Bauch „zu füllig“ (P22:138).

Bei P1 ist seit einer Lungenembolie „nix normal im Körper“ (P1:120). Danach hat sie nämlich $75 \mathrm{~kg}$ zugenommen - ohne eindeutig bestimmbaren Grund (P1:008). Kurz darauf ist die Diagnose gestellt worden. Belastend war es für P1 immer wieder von medizinischem Personal auf ihr Gewicht angesprochen zu werden. „Frustessen“ und der „Jo Jo-Effekt" durch Insulin bewirkten, dass sie nicht abnehmen konnte (P1:016). Seit die Insulindosis reduziert wurde, hat sie ohne anders zu essen abgenommen (P1:020).

P2 hatte vor der Diagnose Diabetes schon Erfahrungen mit anderen Erkrankungen. Diabetes hat er „auf die leichte Schulter“ genommen, da er sich „wohl gefühlt“ hat und „um die Welt reisen“ konnte (P2:029). Der frühere Unternehmer berichtet von Exzessen „mit Fleisch bis zum Abwinken“, die nach beruflichen Erfolgen gefeiert wurden. Diese habe sein Körper ohne "Defekt" gut vertragen (P2:041). Jetzt ist sein "Sonntagsbraten“ und eigentlich „alles verboten“ (P2:055) und „extreme Disziplin“ nötig (P2:019).

P3 erzählt, dass er als schon als Kind wegen seines Übergewichts „gehänselt“ und „ausgelacht“ wurde (P3:167). Die Gewohnheit, viel zu essen bringt er damit in Verbindung, dass er in der Nachkriegsgeneration großgeworden ist, als "die Väter [...] verhungert aus dem Krieg [kamen]“ (P3:153). P3 kritisiert, dass „ständig und überall“ Fast-Food angeboten wird und „so kranke Menschen wie [er] [...] ständig weiter verführt werden [können] (P3:148).

$\begin{array}{lllll}\text { P8 } & \mathrm{m} & 73 & 3 & \text { Orale Therapie. }\end{array}$

P8 betont, dass er weniger übergewichtig war, als die anderen Leute bei der Diabetesschulung, an der er teilgenommen hat (P8:0325). Er nimmt die Ernährungsumstellung sehr ernst, versucht z.B. ohne Salz und Zucker „auszukommen“ (P8:0513). Auf einige Dinge, wie Käse und Rotwein möchte er jedoch nicht verzichten, „denn ein bisschen was muss man ja auch [...] sündigen, sonst hätt[e das] Leben ja gar keinen Zweck mehr“ (0539). Seine Frau hat die Ernährung „in [den] Griff gekriegt“ (P8:0041).

P6 w $52 \quad 25 \quad \begin{aligned} & \text { Basal unterstützte } \\ & \text { orale Therapie. }\end{aligned}$

P6 erzählt, dass er zunahm, als er anfing, im Schichtdienst bei der Bahn zu arbeiten und nicht mehr genügend Bewegung mehr hatte (P6:052). Im Alter von 27 Jahren wurde Diabetes bei ihm festgestellt. Er erhielt einen Diätplan. Seine Mutter und später seine Frau achten auf seine Ernährung. Er betont, wie wichtig ein solcher „Zweiter Wille“ ist (P6:156) - ansonsten kommt der „Schlendrian“ hervor. Für "eine schöne Party“ „reiß[t er] öfters aus“ (P6:350).

\begin{tabular}{cccc}
\hline P27 w $65 \quad 24 \quad \begin{array}{l}\text { Erst orale Therapie, } \\
\text { seit 15 Jahren Insu- } \\
\text { lintherapie. }\end{array}$
\end{tabular}

P27 wurde immer wieder aufgefordert abzunehmen. Sie hat festgestellt, dass sie, auch wenn sie gar nichts isst, trotzdem einen hohen Blutzuckerspiegel hat (P27:010). P27 erzählt von einer Arbeitskollegin, die viel dicker ist als sie und immer heimlich Süßes isst, aber nicht an Diabetes leidet (P27:110). P27 sagt, dass sie selbst nur in seltenen Fällen Süßes isst - im Gegensatz zu ihrem Mann (P27:110).

$\begin{array}{llll}\text { P29 w } 65 & 32 & \begin{array}{l}\text { Erst orale Therapie, } \\ \text { seit } 26 \text { Jahren Insu- }\end{array}\end{array}$ seit 26 Jahren
lintherapie.

P29 erzählt, dass ihr die Umstellung auf die Insulintherapie nicht schwergefallen ist, weil sie als Krankenschwester schon mit Spritzen umgehen konnte (P29:063). Die neue Medikation führte zu „Erfolgserlebnissen“, nämlich „Schöne[n] Werte[n]“ (P29:063). Sie engagiert sich in einer selbstgegründeten Selbsthilfegruppe (P29:131).

\begin{tabular}{lllll}
\hline P15 & w & 73 & 3 & Orale Therapie.
\end{tabular}

Auch Mutter, Schwester und Tanten von P15 hatten Diabetes Typ 2 (P15:0434,0440). Sie selbst hatte schon 20 Jahre lang immer wieder erlebt, dass ihr Blutzucker in Stresssituationen, wie z.B. nach dem Tod ihres Mannes und später des Sohns erhöht war (P15:0273). Seit 3 Jahren wird sie nun medikamentös behandelt und achtet auf ihre Ernährung. P15 fällt es nicht schwer, auf Süßigkeiten zu verzichten (P15:0277). Sie sagt, sie „war immer die Dicke“ und hat darunter schon in der Schule gelitten (P15:0540). 


\subsection{Kernkategorie: Verzicht als beherrschende Erfahrung bei Diabetes}

\section{Typ 2}

Menschen mit Diabetes Typ 2 sind spätestens ab dem Diagnosezeitpunkt mit der Forderung konfrontiert, ihren Lebensstil im Sinne einer Diät und Erhöhung der körperlichen Aktivität zu verändern. Alle Interviewten empfinden dies als Druck, in Zukunft auf bestimmte Genüsse verzichten zu müssen. „Verzicht“ ist das zentrale Phänomen, das im Mittelpunkt der Erfahrung mit Diabetes Typ 2 steht.

Das Thema Verzichten, das sich wie ein „roter Faden“ durch letztlich alle Interviews zieht, wurde zunächst in Form eines Memos charakterisiert, um sich seiner Logik bewusst zu werden. Das Memo findet sich vollständig in Anhang (9.1). Im Folgenden wird nur der Grundgedanke wiedergegeben.

Die Aufforderung, den eigenen Lebensstil zu ändern, ist für viele der Interviewten mit dem Begriff „Verzichten“ verknüpft. Es besteht ein hoher moralischer Druck, auf bestimmte Lebensmittel zu verzichten und Sport zu treiben. Dabei geht es nicht nur um die zukünftige schwierige Aufgabe, das eigene Leben zu ändern, die jeder allein bewältigen muss. Nein, auch der vergangene Lebensstil, das frühere Verhalten und damit der eigene Charakter werden zur Disposition gestellt. Jeder muss sich zu der Frage nach Schuld an der Erkrankung und Verantwortung für den Krankheitsverlauf positionieren. Bei dem Versuch, der Forderung nach Verzicht gerecht zu werden, haben viele der Interviewten den Eindruck, dass sie von der Außenwelt und insbesondere medizinischem Personal nach ihrem Äußeren, also ihren Körpermaßen und ihrem Gewicht und den Laborwerten beurteilt werden - und nicht nach den eigenen Anstrengungen. Etwas aufgeben, dem Genuss entsagen, sich gar gesünder verhalten müssen als „die Gesunden“: diese Aufgaben werden als große Ungerechtigkeit empfunden.

\subsection{Subkategorie 1: Verzicht ist schmerzhaft}

Die Lebensstilveränderung ist für alle Interviewten schmerzhaft. Dies liegt zum einen daran, dass es gerade für sie emotional bedeutsame Objekte zu sein scheinen, auf die sie verzichten müssen. Viele sehen darin eine Gefährdung ihrer Lebensqualität. Im Vergleich mit Mitmenschen, die vermeintlich auf nichts verzichten müssen, fühlen sich viele Interviewte ungerecht behandelt. Dazu kommt der Eindruck, von sozialen Ereignissen ausgeschlossen zu sein. Manche der Interviewten nehmen „Verzicht“ als lebensferne ärztliche Forderung wahr und fühlen sich im Umgang mit ihrer Krankheit alleingelassen. Trotzgefühle und die Ablehnung einer Lebensstilveränderung können die Folge sein.

\subsubsection{Auf ein genussvolles Leben verzichten müssen}

Da die Veränderung der Ernährungs- und Bewegungsgewohnheiten zu den Behandlungszielen bei Diabetes Typ 2 gehört, sind Ärztinnen und Ärzte diejenigen, die viele 
interviewte Personen „offiziell“ zu einer Veränderung ihres Lebensstils auffordern. Als eine Interviewte (P21) von ihrem Arzt dazu angeregt wird, auf bestimmte Lebensmittel zu verzichten, ist sie zunächst nicht damit einverstanden, ihre Gewohnheiten umzustellen.

Und dann hat er empfohlen, also nicht mehr so viel Weißbrot essen und also Kuchen wenn [es] geht sein-. Also, es klang schon sehr, eh, hart. Und hab‘ mich denn doch nicht so daran gehalten. (P21:237)

In diesem Zitat fällt ein Widerspruch auf: Im ersten Satz klingt es zunächst so, als habe P21 die Aussage des Arztes als moderat bewertet. Die Formulierungen „nicht mehr so viel“ und „wenn [es] geht“ wirken wie vergleichsweise milde Einschränkungen. Eine solche ärztliche Aussage sollte wenig Druck ausüben. Überaschenderweise fügt sie aber hinzu, dass die Empfehlung für sie „sehr hart“ klang. Das Wort „hart“ könnte hier als den Arzt charakterisierend gedeutet werden, welcher vielleicht auf P21 „streng“ und "gefühlskalt" wirkte, so als könnte er sich nicht in ihre Lage hineinversetzen. Andererseits kann das Wort „hart“ auch die Ernährungsumstellung betreffen, welche das Leben in eine „starre“ Form zu pressen droht. In einer dritten Lesart kann „hart“ auch als eine Eigenschaft interpretiert werden, die benötigt wird, um sich an eine Diät zu halten. „Hart“ ist dabei ebenso wie „eisern“ - ein Wort mit dem sich P21 später mehrfach im Umgang mit der Diät beschreibt (P21:273; P21:534; P21:554) - sowohl negativ als auch positiv besetzt. Ein "harter“, „eiserner“ Mensch kann sich dadurch auszeichnen, dass er seine Aufgaben kalkuliert, effektiv und ohne sich durch fremde Einflüsse und Reize ablenken zu lassen, erfolgreich ausführt. Gleichzeitig kann ein „harter“ und „eiserner" Mensch sich nicht so flexibel an neue Bedingungen anpassen; er wird eher nicht als empathisch gelten, vielleicht sogar als ungesellig.

P21 hat sich dann auch - weil "es so hart klang“ - „doch nicht so daran gehalten“. Wortwahl, sowie Sprachmelodie und Mimik ${ }^{15}$ vermitteln bei dieser Aussage einen kindlich-trotzigen Eindruck. Ihr scheint es schwer zu fallen, zuzugeben, dass sie die ärztlichen Ratschläge nicht berücksichtigt hat. Möglicherweise schämt sie sich - deshalb die Formulierung „nicht so dran halten“, anstelle von „nicht dran halten“. Gleichzeitig scheint P21 die Forderung nach Veränderung der Ernährungsgewohnheiten als Ungerechtigkeit zu empfinden. Etwas später im Interview wird dies noch deutlicher:

15 Zusätzlich zu den Transkripten der Interviews lagen von einigen Interviews, sofern die Personen dazu ihre Einwilligung gegeben hatten, Videoaufnahmen vor, die in diesem Fall in die Interpretation einbezogen werden konnten. 
Und, wie der Doktor eben sagte, Sie müssen verzichten auf wirklich auf ((stottert)) auf jegliche Weiß- äh, Weizenbrot-Sachen und, [...] also da dacht ich, ist hart, also irgendwie machst du [das] nicht. Oder so, ja ((lachend)) also wirklich, das kann dann nicht sein, dann nur vom Weißbrot und (2) ${ }^{16}$ ja. Also es war, war doch ' $\mathrm{K}$ Knick irgendwie in-in m-meinem ja? ((lachend)) meinem Leben so. [...] Also und eben man hat daran gedacht damals so, weil man frei [war] vorher irgendwie, aber nun (2) hat man doch immer irgendwas zu denken, oder ((lachend)) so. (P21:318)

P21 reagiert mit Trotz auf diese Ungerechtigkeit („also irgendwie machst du [das] nicht“). In der Formulierung „verzichten [...] auf jegliche [...] Weizenbrotsachen“ drückt sich ihre Wahrnehmung aus, die Forderung des Arztes sei übertrieben. Sie erlebt die ärztliche Aussage als diffus und ungenau. Zugleich hat sie das Gefühl, dass ihr die Freiheit oder das Recht auf diesen gewohnten Genuss entzogen wird und sie weniger unbeschwert leben kann („nun (2) hat man doch immer irgendwas zu denken“). Diese Unfreiheit scheint umso ungerechter, da es ihr spürbar „gut geht“ (vgl. Kap. 5.3.3).

Hier bestätigt sich die Annahme, dass „Verzicht“ als „hart“ erlebt wird, als starre Vorgabe durch unnachgiebiges medizinisches Personal und als wenig an den Alltag - z.B. die Fülle an diversen Weißbrotsorten bei P21 - angepasst. Ein anderer Interviewter äußert das Gefühl, ungerecht behandelt zu werden besonders deutlich:

Dann werden Sie wirklich von einem Ernährungsseminar zum nächsten geschickt [...].Nachher hängt Ihnen das Zeug- [...] Die erzählen immer das Gleiche, ich darf kein Bier mehr trinken, ich darf keine Schweinshaxe mehr essen, äh, mein Sonntagsbraten ist verboten und, und, und, äh, mir wird alles verboten, grundsätzlich ist das so, das ist nämlich das andere Extrem davon, ich darf überhaupt nichts, ich geh doch nach Hause, die können mich doch mal, so, okay, es wird genau das Gegenteil von dem erzielt, was man eigentlich will. (P2:055)

P2 hat den Eindruck, dass inm „alles verboten“ ist. Er hat den Eindruck, dass ihm als Menschen mit Diabetes „grundsätzlich“ kein Genuss gegönnt wird. Die Aufforderung zur Lebensänderung wird als Lebensbedrohung wahrgenommen. Es scheinen gerade Dinge verboten zu werden, die ihm emotional wichtig sind. Er nimmt ein strenges Regelwerk wahr, das von einer medizinischen Autorität festgelegt worden ist, die seine Lebenswelt nicht kennt. Das rechtfertigt - für ihn - eine Trotzreaktion.

\subsubsection{Benachteiligt sein}

Verzicht kollidiert mit sozialen Anlässen, die traditionell von bestimmten Ernährungsgewohnheiten geprägt sind, z.B. mit Einladungen oder Festen. Einer Interviewten fällt

\footnotetext{
${ }^{16}$ Bei den eingeklammerten Zahlen handelt es sich um die Angabe, wie viele Sekunden die interviewte Person zwischen Sätzen oder Wörtern geschwiegen hat. Diese Erzählverzögerungen können, genau wie die Wiederholung von Silben („Stottern“) oder in doppelten Klammern $[((\ldots))]$ angegebene Gesten oder Lachen, Räuspern etc., wichtig für das Verständnis der Erzählung sein oder Hinweise auf emotional bedeutsame Stellen geben
} 
es sehr schwer, auf den Genuss der „wohlverdienten“ Kaffeerunde mit Kuchen zu verzichten.

Und gerade so jetzt im Rentenalter habe ich mich eigentlich auf diese Nachmittagskaffeerunde gefreut. [...] Ja und die, also hat mir denn auch 'ne Kollegin gesagt, nun nimm es mal nicht so ernst und so, aber ich hab's wirklich gelassen. Ja, mit dem Kuchen. Aber es fällt mir schwer, ja. Nachmittags dann irgend ' $n$ Knäckebrot zu knabbern. (P21:265-269)

Aber irgendwie im Hinterkopf ist immer, wenn andere jahrelang doch zum Kaffee gehen (...), dann- ist man doch ' $\mathrm{n}$ bisschen handi- gehandicapt. (P21:334)

In diese Enttäuschung hinein spielt vermutlich eine lebenslange Erwartung, es sich im Alter gut gehen zu lassen, sich zu belohnen und nicht mehr disziplinieren zu müssen. Die genannte Alternative zum Kuchen - „irgend 'n Knäckebrot knabbern“ - klingt trostlos. Durch die Formulierung, sie sei „gehandicapt“, veranschaulicht P21, dass sie sich aufgrund ihres „Anders-Seins“ eingeschränkt fühlt, wohlmöglich auch benachteiligt. An anderer Stelle zeigt P21 ihre Enttäuschung über die Ungerechtigkeit an Diabetes erkrankt zu sein, weil sie doch - überspitzt ausgedrückt - im Vergleich mit anderen zu „den Guten“ gehört.

[...] ja, war ich auch immer schockiert, und Kolleginnen und Freundinnen die beleibter sind und, eh, haben nichts und ich als schlanke Person, sag ich mal, haben da diese hohen Werte, ja? (P21:622)

Hier klingt eine Kategorisierung in schlank und übergewichtig an, ein Thema, das in Kap. 5.5.2 näher betrachtet wird.

Obwohl „Verzicht“ eine häufig von außen herangetragene Erwartung ist, gibt es gleichzeitig auch gegenteilige Forderungen: einen starken Anpassungsdruck und -wunsch vom eigenen sozialen Umfeld, weiterhin so wie früher zu leben. Das erschwert das Verzichten. Im Fall einer Interviewten (P30) bedeutet das, für ihre Kinder und ihren Partner „ungesunde“ Gerichte zu kochen. Sie muss sich beherrschen, während die anderen unbeschwert „sündigen“ und nicht Diabetes Typ 2 haben. Diese Ungerechtigkeit löst in ihr ein einen starken Widerwillen, einen „Ekel“ aus, insbesondere, da sie sich von ihrem Partner unverstanden fühlt.

Dann wieder die Fragen [...], „Was gibt es zum Essen?" - von den Kindern. Es kommt ja immer, ständig diese Frage: „Was gibt es?" [...] Die essen nun nämlich auch alles. Sprich, also, ich koche eigentlich das, was die am liebsten mögen. Und das ist leider nicht immer das gesündeste. [...] und mehrere Sachen zu kochen, dass ich praktisch für mich alleine koche, ist irgendwie blöd [...]. Jetzt versuche ich schon immer, so eine gesunde Richtung einzuschlagen. Aber da stellen sie sich immer quer. [...] Auch mein Mann. Der stellt sich manchmal ganz arg quer. Weil er ist so ein Typ, er macht viel mit Butter, und, und, und, die schön deftigen Sachen. Und ich versuche das alles, zu reduzieren. Sage ich: "Du, es geht auch mit weniger. Versuche es mal." ((lacht)) Es ist nicht einfach, dagegen anzukämpfen. Dann sagt er wieder: "Hmm, das schmeckt mir nicht." So andersherum, wenn er kochen 
soll, dann sage ich auch: "Hm, schmeckt mir nicht, weil es mir zu fettig ist", ne. Und, ähm, das ist dann auch nicht einfach in der, also selbst in der eigenen Familie dann da einen Einklang zu bekommen. Weil er ja weiß, äh, ich bin zwar krank, ich dürfte vieles nicht, aber das interessiert ihn nicht. Er will sein Essen haben, er will das, was ihm schmeckt. (P30:252-256)

Während P30 in diesem Zitat darstellt, dass es vor allem die Erwartungen und Bedürfnisse anderer sind, gegen die sie den Willen zu verzichten verteidigen muss, beschreibt sie an andere Stelle ihren inneren Kampf gegen eigene Bedürfnisse und Verführungen. Wenn ihre Tochter ihr empfiehlt, nicht an Essen zu denken, hilft ihr das gar nicht und unterstreicht noch die Ungerechtigkeit, dass diese ein lockeres Verhältnis zu Essen hat, während sich P30 selbst so quält. Das fast zwanghafte „Kreisen“ um das Thema „Verzicht“ ist gewissermaßen eine Steigerung des von P21 angesprochenen Gefühls, nicht mehr unbeschwert leben zu können: „nun hat man doch immer irgendwas zu denken“ (P21:318).

Und wenn man dann so sieht: die Kinder kommen nach Hause, die essen was, und so, und Du hattest schon Deine Portion. "Nein, es darf nicht genascht werden!" Aber man kommt in der Küche vorbei: Hmm, einmal kurz Topfgucken. Oh, da ist noch was drin. Und schwupps ist schon wieder was drin. Und das geht, manchmal sage ich mir, ich müsste eigentlich die Küche absperren, um einfach nicht-. Weil, wenn der Gedanke da ist, dass ich weiß, ich habe da was, dann bohrt das sich unwahrscheinlich tief rein, und: Hmm, das Verlangen wird immer größer und größer, und Du bist dann da und: "Nein, Du darfst nicht!" Das ist ein ewiger Kampf, den man schon seit Jahren eigentlich kämpft. Und weil meine Tochter sagt auch: "Wieso? Ist doch ganz einfach: Denk doch nicht dran!" Naja, toll! Ich denke aber an nichts anderes mehr. Weil, weil das immer, ständig, ähm, in einem kreist, äh: Was dürfte ich? Was könnte ich? (P30:248)

Indem P30 vorschlägt, die Küche „abzusperren“, erklärt sie ihren Wunsch, sich nicht ununterbrochen mit Verzicht auseinandersetzen zu müssen. Sie braucht die Tür als "Grenze“, die sie vor Verführungen schützt.

So wie „Verzicht“ vom sozialen Umfeld als Forderung an die Patientin herangetragen wird, kann es auch sein, dass das soziale Umfeld die Bemühungen um Verzicht unterläuft, besonders deutlich bei Festen:

Wenn man eben halt auf, ähm, Feierlichkeiten ist, und sagt: "Nein, man trinkt keinen Alkohol [...]Und wenn ich dann jetzt jedes Mal erklären muss, so wie jetzt, es war Wiesenanstich gewesen bei uns, in unserer Wirtschaft [...] ich dann mein Wasser bestellt habe, und die mich alle angucken: "Willst Du nicht Dein Bierchen haben?" Sage ich: "Nein, will ich nicht [...]. Aber es wird jedes Mal von den anderen immer, so irgendein schräger Blick kommt schon manchmal, wenn man in dieser Gemeinschaft nicht mittrinkt, oder mitisst. Weil, wenn man dann sagt: "Nein, ich hatte meine Portion. Es langt. [...] Ihr wisst, ich habe Zucker. [...]“ Aber die, die reiten drauf rum.

Warum andere Leute das immer so machen? Keine Ahnung. [...] Vielleicht um auf ihr, äh, ihr Essen da eben halt, was die da in sich- vielleicht zu beschönigen. [...] Also, ich bin standhaft, ich, also, das sage ich auch jedes Mal den Ärzten [...]. "Ihr, ihr wisst gar nicht, wie, wie schwierig das ist, manchmal für mich, da zu stehen, und, ähm, drauf zu verzichten." [...] Also es ist jedes Mal ein Kampf aufs Neue und nicht einfach. (P30:093-108) 
Für das Unverständnis, dass P30 entgegenschlägt, wenn sie in Gesellschaft nicht mitisst und -trinkt, hat sie eine besondere Erklärung: Es gefalle anderen nicht, sie verzichten zu sehen, weil sie ihren eigenen „ungesunden“ Lebensstil dann in Zweifel gezogen sehen. Wie ihr Partner und ihre Kinder "sündigen“ auch ihre Bekannten. Man spürt deutlich den Versuch von P30, ihr Verhalten im Kontrast zu den anderen als "richtig“ zu beschreiben. Diese eigene moralische Erhöhung tröstet P30 möglicherweise bei dem Gefühl, benachteiligt zu sein.

Der Ausschluss von "normalen“ sozialen Essgewohnheiten ist schmerzlich und kann für die sozialen Beziehungen schädlich sein.

Auch wenn man so am Wochenende sich dann getroffen hat, auch, äh, Bekannte eingeladen hat, das, das war, das entfiel. Vieles entfiel. Weil es ging einfach nicht, auch für mich nicht. Äh, wenn man da sitzt, alle essen, und dann, Du hast dann Deinen [Diät-]Shake in der Hand, das ist sehr ungewöhnlich, auch für-. Wenn ich einen Gast einlade, ist das bei uns, äh, sehr, einen Gast einzuladen, den Gast musst Du bewirten und da-. Wenn Du nichts isst, dann denkt der: "Was ist jetzt los?" (P32:701)

P16 entscheidet sich nach der Diagnose Diabetes gegen eine weitere Teilnahme am Stammtisch, weil sich Verzicht für inn nicht mit der Idee eines Stammtischs vereinbaren lässt. Für inn sind Essen und soziale Interaktion kaum voneinander zu trennen.

$\mathrm{Na} \mathrm{ja,} \mathrm{gut} \mathrm{mit} \mathrm{der} \mathrm{Gewohnheit} \mathrm{da} \mathrm{geht} \mathrm{das} \mathrm{also} \mathrm{los,} \mathrm{äh,} \mathrm{zum} \mathrm{Beispiel} \mathrm{Stammtisch} \mathrm{äh,} \mathrm{wo} \mathrm{man} \mathrm{also} \mathrm{hingegangen}$ ist, hat sonst 'n paar schöne Bierchen irgendwo getrunken [...]. Ich will abnehmen, ich äh, ich geh nicht essen, ich geh nicht in eine Kneipe. Das geht also einfach nicht, ich krieg das nicht auf die Reihe, ne, weil, das passt also einfach nicht, ääh, eben halt, äh, auf der einen Seite diese drei Broteinheiten essen und auf der andern Seite in 'ner Kneipe, äh, essen so ' $n$ Teller voll oder so und dann hab ich gesagt, dann bleib ich lieber zuhause. (P16:08260828)

Das Gefühl, durch den Verzicht von sozialen Ereignissen - innerhalb und außerhalb des Familienlebens - ausgeschlossen zu werden oder sich selbst zum Schutz vor „Verführungen“ ausschließen zu müssen, spielt eine große Rolle im Alltag.

Familie [ist] sehr, sehr wichtig für mich, dass sie mich dabei unterstützt. Sie tut es, auch in, bei diesem [Diät-]Programm. [In den] Anfangszeiten, dass sie dann in der Küche gegessen haben, dass ich dann nicht, äh, Appetit gekriegt habe. Das war so. Auch, äh, in diesen drei Monaten, wo ich nichts gegessen habe, hat das Familienleben ein bisschen [gelitten]. Weil am Wochenende war es, ist Tradition, dass man am Frühstückstisch sitzt, alle gemeinsam, wo man dann gefrühstückt hat, wo man dann, äh, erzählt hat, die Woche, was alles passiert. Das entfiel. Das war auch für sie eine Einschränkung, für meine Familie. Für mich sowieso. (P32:697)

Diese Passage bezieht sich auf die Zeit, in der P32 während eines ärztlich begleiteten Diätprogramms nur Trinknahrung zu sich nehmen durfte. In dieser Passage deutet P32 an, dass er eine räumliche Trennung von seiner Familie, die alles essen durfte, brauchte, um sich vor seinem Appetit zu schützen. Dadurch wird aber das Gefühl, ausgeschlossen zu sein, richtig greifbar. 


\subsubsection{Gegen das eigene Wohlbefinden kämpfen müssen}

Verzichten bei Diabetes scheint besonders schwer zu sein, da die Krankheit in den meisten Fällen nicht spürbar ist. P21 erzählt, dass es ihr anfangs geradezu unlogisch erschien, sich einzuschränken, obwohl es ihr spürbar gutging.

Interviewerin: Ihnen ist ja gesagt worden, eh, Weißbrot sollte eher weggelassen werden? [...] Können sie mir sagenWie sie das empfunden haben?

P21: Tja, ne es klang schon sehr, sehr hart für mich, ja? Das ich, also irgendwas weglassen soll, obwohl es mir gutgeht. (P21:612-618)

Viele Interviewte begründen mit diesem Phänomen, dass sie die Anweisung, ihr Leben zu verändern, gerade zu Beginn der Erkrankung nicht ernst genommen haben.

Wenn Sie nun nicht gerade ein Bein gebrochen haben und mit Gips daherkommen oder wenn Sie wie in dem anderen Falle einen Autounfall erleiden, dann können Sie halt nicht anders, dann werden Sie in so ein Bett gepackt und dann war's das erst mal, dann sind Sie weg von der Welt [...], mhhm, dann glaubt man das. Hierbei ist es tatsächlich so, äh, mir geht es so, [...] ömh, hab das sehr auf die leichte Schulter genommen, weil, das war- zu Beginn waren das keine, keine, äh, großen körperlichen Dinge, obwohl man natürlich, äh, sich kaputt gefühlt hat, obwohl man Schwierigkeiten hatte, mh, der der Blutdruck war durcheinander, äh, und die- man hatte Konzentrationsschwierigkeiten [...]. [Habe] hier in [Name einer Stadt] eine schwere Krebsoperation gehabt [...]. Sie sehen also, wenn man all diese Dinge überlebt hat, [...] [Sie] sich immer noch einigermaßen wohl fühlen und, und, und um die Welt reisen können, ich hab in der Zwischenzeit eine Firma in China gegründet, wo 400 Leute beschäftigt sind, äh, [...] und wenn Sie das alles, äh, noch so locker flockig, öhm, nebenbei machen können, wissen Sie, dann kann das mit der Diabetes nicht so weit hallen, also nehmen Sie das auf die leichte Schulter. (P2:029)

In dieser Passage wird deutlich, was Diabetes von anderen Erkrankungen unterscheidet: der Interviewte hat bereits Erfahrungen mit einem schweren Autounfall und einer Operation wegen einer Krebserkrankung gemacht, sodass er spürbar beeinträchtigt war und durch eine Isolation im Krankenbett automatisch nicht so weiterleben konnte wie zuvor. Diese akuten Leiden waren Grenzen, gegen die er aufbegehren konnte „Wenn man das überlebt hat, [Sie] sich immer noch einigermaßen wohl fühlen und, und, und um die Welt reisen können" - und gegen die er sein normales, aktives Leben verteidigen konnte. Diabetes dagegen schränkt inn zunächst wenig ein. Er schildert zwar, dass er spürbare Ermüdungserscheinungen hatte, aber leistungsstark genug war, um eine Firma zu gründen. Diabetes bietet keine Angriffsfläche, keine äußeren Einschränkungen, die er ausreizen kann, sondern alle Grenzen muss er sich als kranke Person selbst auferlegen. Aus der folgenden Aussage eines anderen Interviewten, lässt sich ablesen, wie schwerwiegend die fehlende Spürbarkeit von Diabetes sich auf den Willen zu verzichten auswirken kann. P16 schildert, wie Menschen Medikamente als bequeme Alternative zur Lebensstiländerung verstehen, grenzt sich aber von diesen $a b$ : 
Nur eben halt dieses Gewicht, ne, und da muss man dann eben halt auch permanent mit kämpfen [...] Man muss eben halt nur, sehr diszipliniert sein [...] Die meisten sagen dann wahrscheinlich irgendwann: „Mensch ich hab kein Bock mehr" [...] Es tut ja nicht weh, man merkt es nicht und, naja viele sagen dann eben halt naja äh was soll's, äh, dann nehme ich eben halt äh 'ne Tablette oder zwei oder auch drei, äh und manche sagen auch, äh, „Bevor ich das also dann eben halt äh anders mache, denn spritz ich." (P16:1430-1463)

Wie P2 kontrastiert P32 Diabetes mit erlebten spürbaren Erkrankungen:

Es war so wie, ja, es, die Krankheit war - wie soll ich das ausdrücken? Die Krankheit war, ich habe nichts gespürt. Also, dass ich krank war. Magenschmerzen hatte ich. Das war krank. Ich hatte Schmerzen. Da war was. (P32:226)

Erst ein Herzinfarkt stellt für P32 einen Wendepunkt innerhalb der Diabeteserkrankung dar, welcher in ihm der Ehrgeiz weckt, seine Lebensgewohnheiten zu verändern.

Ja, und ab da war es mir bewusst, dass ich krank bin, und dass der Diabetes, äh - ich spürte inn nicht wie immer noch - dass ich zuckerkrank bin. Aber die Folgeerscheinungen, Blutdruck, Herzinfarkt, die waren richtig, richtig heftig. (P32:234)

Ein Interviewter erlebt einen Wendepunkt auf andere Weise. Er muss die Folgeschäden nicht selbst spüren. Er hört bei einer Ernährungsschulung, dass ein Mitpatient mit schon gravierenden Folgeschäden durch den Diabetes danach fragt, wie er weiterhin ungesunde Lebensmittel zu sich nehmen kann - und ist abgestoßen. Mit diesem Menschen will P16 nichts gemeinsam haben.

Ich hab das also, mh, da gesehen bei der Ernährungsberatung. [...] Ich kann mich also an einen erinnern der saß da, also der hatte, äh, der hatte schon zwei Zehen ab. Der saß im Rollstuhl hatte so einen äh, dicken Fuß, blau, grün, rot, äh mit einer Kanüle drin äh, wo entwässert worden ist und der war also kurz vor der Amputation. Und der ähm, der hatte also wirklich nur im Kopf, wie kann ich das also machen oder was muss ich also machen, ähm, um eben halt meinen Sahnekuchen zu essen und mein Bier zu trinken und [...] das hat mich also, sag ich mal, stark auch beeinflusst, ähm, dass ich also gesagt habe [...], wenn ich also so 'ne Situation vermeiden kann, für mich selber, dann will ich also gerne alles dafür tun was also möglich ist [...] [lch] wusste eben halt was alles passieren kann ja und dann hab ich das aber da in der Wirklichkeit gesehen. (P16:0581-0616)

Während die meisten Erkrankten ihren Diabetes als nicht spürbar erleben, erzählen manche der Interviewten, dass sie die Höhe der Werte fühlen können. Einige verifizieren dann ihre körperliche Wahrnehmung einer Unterzuckerung durch das Messen.

Wenn wir irgendwo unterwegs sind und ich kriege so 'nen Hungerast, dann messe ich mal und dann sage ich, ach, mal 'nen Stück Schokolade essen oder so was. (P6:129)

P3 kann keine Unterschiede zwischen verschiedenen Zuckerspiegeln spüren. Einzig seine Stimmung diene inm manchmal als Hinweis für seine schwankenden Blutzuckerspiegel.

Zu dem 500 Zucker und jetzt zu dem 100 Zucker, da empfind ich keinen großen Unterschied, so, so wie's mir geht, [...] das einzige, was vielleicht, ja, messbar ist, ist das ich vielleicht, dass ich, dass ich ruhiger, ne- so nicht so genervt bin, das ist die einzige, die einzige Sache, wo, wo ich meine, wo man das selber messen kann, finden kann, wie es einem geht mit Zucker, ich bin der Meinung, dass Diabetes, das merkt man nicht. (P3:072) 
P3 sucht nach einem spürbaren Korrelat seiner Werte, nach etwas, das er „selbst messen“ kann und das vielleicht den Aufwand rechtfertigt, den Blutzucker zu senken. Er findet interessanterweise keine körperlichen Symptome, wie z.B. Schmerz, sondern emotionale Zeichen. Ein zu hoher Zuckerspiegel scheint das subjektive Wohlbefinden einzuschränken. Gegenteilige Erfahrungen macht P6: Er schildert, wie er weit davon entfernt war, auf etwas zu verzichten zu wollen, da er bei hohen Werten ein Wohlgefühl verspürte, das er mit einer Drogenwirkung vergleicht:

\begin{abstract}
Also dieser, dieser, dieser Prozess, dass man da, äh, zunimmt, der dauert eigentlich ziemlich lange. Man bemerkt es auch gar nicht. 'Ne neue Hose kaufen. Ja, och, nimm 'ne Nummer größer. Ist doch egal. [...] Ich will nicht sagen man wird faul dabei oder man wird, wird träge oder gaga. Gleichgültig ist es vielleicht. Es interessiert einen nicht. Man fühlt sich sauwohl. Und ich habe später dann mal festgestellt, je höher der Zuckerspiegel war, als wenn man 'nen Junkie wird, man fühlt sich immer wohler. Man fühlt sich mit 'nem Zuckerwert von über dreihundert wohler als wenn ich nur hundert habe und, und krieg 'n Hunger als nichts raus. Das ist zwar nur 'n so 'n, so 'n, so 'n Kopfgefühl oder, oder 'n, oder 'n, ob man da angeführt wird mit, mit irgendwas oder irgendwoher. (P6:056-059)
\end{abstract}

Im Gegensatz hohen Blutzuckerspiegeln, ist „Verzicht“ (schmerzlich) spürbar: Einige Interviewte klagen über ein ständiges Hungergefühl, das sie stets über Essen und über den Druck, nicht essen zu dürfen, nachdenken lässt.

Ja, viel, viel Wissenschaft ist da, mit drin: Was darf ich wann essen? Und, und, und. Das ist, und dann sage ich immer: "Ach, von so einer kleinen Portion [soll ich satt werden]? [...] Das ist ein ganz großes Problem, weil, weil da ständig immer ein Hungergefühl ist. (P30:248)

Als ähnlich beherrschend schildert P16 sein „spürbares“ Erleben von Verzicht.

Und irgendwo, hat man permanent Hunger. (P16:1226)

Viele Interviewpartner klagen darüber, dass Insulin den Hunger noch verstärkt. Daraus ergibt sich angesichts des Drucks zu verzichten ein quälender Teufelskreis, aus dem sie auch mit gutem Willen kaum entkommen können.

Und je mehr ich spritze, umso mehr Hunger krieg ich, also muss ich auch mehr essen. (P1:581)

\title{
5.4.4 Allein gelassen werden
}

Der Kontrast zwischen den Auflagen des medizinischen Personals und der lebensweltlichen Umsetzbarkeit scheint groß. Eine Interviewte formuliert mehrfach den Unmut darüber, dass die ärztliche Behandlung darauf beschränkt sei, sie, die Patientin, zum Verzicht aufzurufen - ohne konkrete Hinweise, wie.

Vom Arzt kriegt man immer nur zu hören: "Sie sind jetzt Diabetikerin. Machen Sie die Schulung und halten sich an Ihre Diät." Wums. Aber das alles drum herum, das, das, das kriegt man eigentlich nicht vom Arzt selber direkt 
mitgeteilt. Sondern eigentlich mehr von den Schwestern oder eben halt die Apotheker, oder von den Selbsthilfegruppen. Oder man sucht es sich alles selber raus. Aber von den Ärzten selber kriegt man eigentlich die wenigsten Informationen. (P30:385-391)

Ähnlich vermutet P2, dass es sich die - an ihrem Behandlungserfolg interessierten Ärztinnen und Ärzte - leicht damit machen, Menschen mit Diabetes Typ 2 zur Gewichtsabnahme aufzufordern und sich dadurch letztlich selbst zu behandeln. Er deutet an, dass er mit der Umsetzung der Lebensstiländerung allein gelassen wird.

Nur ich kann einfach nur sagen, dass das bei allen Gesprächen in, äh, medizinischer Umgebung, in medizinischer Runde ein, äh, Standardthema ist, ein generelles Thema ist von allen Medizinern. Alle drängen und drücken darauf und egal welche Fakultät, alle sind sich da einig, dass sie da an der Stelle das erreichen, denn, äh, was können sie groß machen? Eh, jeder ist natürlich auch ' $n$ bisschen am Erfolg interessiert und, ähm, es ist ja am einfachsten wenn man reinkommt und da sagt man erst mal: „Nimm du erst mal 10, 15 Kilo ab, dann, äh, dann geht's dir auch besser." Ist auch klar, ist sehr schön, ist richtig, stimmt, sagen Sie mir, wie ich das machen kann, dann mach ich das. (P2:055)

Der Druck, sich und sein Ernährungsverhalten zu ändern, und der offensichtliche Frust über die ärztliche Behandlung gehen hier eine merkwürdige Paarung ein. Wären Ärztinnen und Ärzte - wie P2 behauptet - am Erfolg interessiert, müssten sie ja gerade inn und andere übergewichtige Personen sensibel beraten und betreuen. Das aber spricht P2 innen pauschal ab. Die Widersprüchlichkeit geht noch weiter: auch, wenn scheinbar genaueste Angaben gemacht werden, auf welche Lebensmittel zu verzichten ist, ist P2 nicht zufrieden, wie sich in der bereits zitierten Passage (vgl. Kap. 5.3.1) zeigte: Die ewige Wiederholung der Diätberatungen führen nur zu dem Eindruck, dass „alles verboten" ist und resultiert in Trotz:

...mir wird alles verboten, grundsätzlich ist das so, das ist nämlich das andere Extrem davon, ich darf überhaupt nichts, ich geh doch nach Hause, die können mich doch mal... (P2:055)

P30 schildert ihren Eindruck, aufgrund ihres jungen Alters keine typische Patientin zu sein und sogar bei spürbaren „Beschwerden“ keine individuelle Aufmerksamkeit zu erhalten, sondern sich selbst behandeln zu müssen.

Genauso wie mit der Herzgeschichte, wo die sagen, dass da irgend so ein Druck zu hoch ist, und sagen: "Das, Sie sind zu jung dafür." ((lacht)) Und dann sage ich noch: "Ja, und was machen wir dagegen?" "Ja, nichts." Und dann ich, äh: "Toll." ((lacht)) Man hat seine Beschwerden. Und dann kriegt man immer zu hören, entweder man soll mehr Sport oder nichts tun. [...] Entweder habe ich immer die verkehrten Ärzte, die nicht zuhören wollen, oder die keine Zeit haben, oder, oder, oder. (P30:067)

Die Empfehlung, diszipliniert Sport zu treiben - für sie auch eine Art von Verzicht -, scheint überhaupt nicht die Erwartungen von P30 an das medizinische Personal zu erfüllen. Während sie eine ärztliche Behandlung erwartet, soll sie selbst handeln. 
Die Erfahrung, dass ihr über Jahre hinweg nur die Empfehlung gegeben wird, sie sollte sich mehr um Verzicht bemühen, ohne dass ein Erfolg sichtbar wird, ist sehr frustrierend und lässt P30 an der ärztlichen Kompetenz zweifeln:

Die Ärzte sind auch alle unterschiedlich. Die sagen immer [...]: "Ja, Sie sind jung. Versuchen Sie es noch aus eigener Sache [...] zu machen." Aber wenn man es über die Jahre sieht, dass diese Werte zu hoch sind, und es klappt nichts. Wo die sich dann vielleicht sagen: „Ach, vielleicht hält sie sich ja nicht an ihre Diät." Und, ähm, da sage ich mir, also, was soll ich noch alles machen. [...]. Also alle, die Patienten sind, die schütteln alle den Kopf, wenn die das hören. Die Ärzte, die schütteln nicht den Kopf, die sagen nur: "Ja, pff, machen wir nichts." Und dann steht man als Patient da und sagt sich: "Hm, warum war ich jetzt eigentlich beim Arzt?" ( P30:213)

In diesem zuletzt zitierten Interviewausschnitt deutet sich eine ganz entscheidende Eigenschaft von Verzicht an: ob genug verzichtet wird oder wurde, wird bewertet. P30 hat den Eindruck, dass sie aufgrund ihrer hohen Blutzuckerwerte "verdächtigt" wird, nicht zu verzichten. (,Wo die sich dann vielleicht sagen: „Ach, vielleicht hält sie sich ja nicht an ihre Diät."). Diese Beurteilung von Verzicht ist von großer Bedeutung für die Erfahrung mit Diabetes Typ 2 und wird in den folgenden Kapiteln behandelt.

\subsection{Subkategorie 2: Verzicht wird moralisch bewertet}

Der Lebensstil der Interviewten, die auf ungesundes Essen verzichten und sich mehr bewegen sollen, wird immer wieder bewertet und zwar nach „sichtbaren“ und „messbaren" Kriterien: nach Körpermaßen bzw. Gewicht und Laborwerten. Dabei wird nicht erst das Verhalten nach der Diagnose beurteilt, auch der frühere Lebensstil wird zur Disposition gestellt. Alle Betroffenen müssen zu der Frage nach Schuld an der Erkrankung Stellung beziehen.

Bei ärztlichen Kontrollterminen ist die Beurteilung des Lebensstils nach diesen Parametern zentral. Einige der Interviewten, deren Laborwerte und Gewicht den Zielwerten entsprechen bzw. sich diesen nähern, werden für ihr "gutes“ Verhalten gelobt. Der Druck, auf Genuss zu verzichten, kann dann etwas geringer werden. Interviewte mit „schlechten“ Werten erleben dagegen eine schmerzhafte Verurteilung, nicht genügend verzichtet zu haben.

Hier wird die starke moralische Dimension von Verzicht deutlich, also die Bewertung des Verhaltens als "richtig“ oder "falsch“. Die Beurteilung erfolgt nicht nur von außen durch Mitmenschen oder medizinisches Personal, sondern auch durch die Betreffen- 
den selbst. Gerade beim Thema Körpermaße ist die verinnerlichte Analogie der Kategorisierung in übergewichtige - maßlose - und schlanke - genügsame - Menschen übermächtig. Vom Aussehen wird auf das Verhalten und damit auf den Charakter geschlossen. Viele der Interviewten beklagen in dieser Vorgehensweise eine fehlende Abbildung inrer Anstrengungen und verweisen auf mangelnde Beeinflussbarkeit des Gewichts oder der Laborwerte. Die Angst vor moralischer Diskreditierung bei „schlechten“ Laborwerten und Übergewicht führt zu einem großen Druck, das eigene Verhalten als verantwortungsvoll zu beschreiben und zu einem Abgrenzungsbedürfnis von „maßlosen" Menschen, die vermeintlich schuld an ihrer Krankheit sind.

\subsubsection{Laborwerte und Körpermaße als „sichtbare“ moralische Prüfmarken}

Erwartungsgemäß prägen bei einer Stoffwechselkrankheit wie Diabetes Typ 2 Laborwerte die Erfahrungen mit ärztlichen Kontrollterminen. Die Arzt-Patient-Beziehung scheint in einigen Interviewpassagen vergleichbar mit einer Lehrer-Schüler-Beziehung. Die Laborwerte erinnern an Schulnoten, die vergeben werden, um Verhalten und Leistung zu bewerten. Das Erreichen günstiger Blutzuckerspiegel („Noten“) wird wie ein Beweis für gelungene Selbstbeherrschung verhandelt. So erzählt P21 davon, dass es aufregend für sie ist, die Laborwerte zu erfahren. Eine ihrer Erzählungen weckt Assoziationen mit einer Zeugnisvergabe, bei der auch eine gewisse Abhängigkeit der Patientin vom ärztlichen Urteil auffällt. Indem sie vom Lob der Ärztin berichtet, stellt sich P21 indirekt als fleißig und diszipliniert dar.

\footnotetext{
$\mathrm{Ja}$, ja ist schon spannend für mich. Also als erstes meine Werte zu erfahren. Und die hat das denn im Computer und [ich] bin dann richtig heiß drauf, zu erfahren, ist es nun gleich oder ist es nicht höher, Gott sei Dank, und war ja bis jetzt immer positive Nachrichten. Und sie [ist], äh, auch immer erfreut darüber. ((lacht)) Und [...] bestärkt mich, weiter zu machen und, wie gesagt manchmal sogar- „Also sie brauchen nicht zu darben.“ Ja? Sie. Gönnen sie sich ruhig was ((lacht)) Es hörte sich dann immer so, so streng an, wie ich mit mir umgehe. (21:570)
}

Daneben zeigt sich in diesem Zitat, dass das Thema Verzichten nicht selten religiösmythologische Züge aufweisen kann. Mehrere Interviewte sprechen von „sündigen“ im Zusammenhang mit ungesundem Verhalten (P3:148; P8:0539; P30:264). Die Formulierung „darben“, die P21 gleich zweimal gewählt hat (P21:334; P21:570), lässt an eine Form der Buße denken, die sie durch Verzicht leisten muss. Sie möchte sich unbedingt als enthaltsam darstellen. Gerade weil ihre Bemühungen so asketisch wirken, wird ihre 
Verpflichtung zu ständigem Verzicht durch die Ärztin erlassen: sie soll sich etwas „gönnen“ - ihr strenger Verzicht wird belohnt. Die ärztliche Kontrolle wird als Beurteilung der eigenen Person wahrgenommen. Dies deckt sich mit der Erfahrung von P16:

Aber, Blutwerte sind also auch alle normal, alle irgendwo im normalen Bereich. Äh, Blutdruck ist im normalen Bereich. Also ich äh, denke mal, dass ich das also durch die Maßnahmen, die ich für mich selber getroffen habe, ganz gut in Griff gekriegt habe und das bestätigt das also auch, was mein Hausarzt sagt. Der sagt, also immer, ich wär sein Musterpatient. (P16:1482)

Während P21 und P16, deren Laborwerte den Therapiezielen entsprechen, die persönliche Bewertung eindeutig positiv erleben, zeigt das folgende Beispiel den Stress, den ärztliche Kontrolle bei schlechten Messwerten auslösen kann.

Weil das ist, ist der Wahnsinn, wenn Du da sitzt vor diesem Arzt und dann, ähm, ja, Du kriegst eigentlich immer nur einen drauf. Weil: Zuckerwerte sind schlecht. Cholesterinwerte sind schlecht. Harnsäure ist schlecht. Triglyceride sind schlecht. So, jetzt sind alle schlecht. (P30:335)

Noch drastischer schildert P1 die Verurteilung durch ihren Arzt anhand der Werte:

P1: Joa, ganz normaler Termin. Das ist einmal quartalsmäßig, da muss ich's erst- erste Mal morgens nüchtern hin, zum Blutabnehmen und zwei Tage später muss ich dann eben zur Besprechung. Und da bin ich das letzte halbe Jahr schon immer mit aufstehenden Haaren hingegangen, ich da- weil ich immer gedacht habe: Was kommt jetzt wieder, ne. [...] Denn man sieht es ja auch, wenn man selber gemessen hat, und man hat wirklich nur hohe Werte, dann kann auch der [Langzeit-] Wert nicht besonders gut sein, ne. Ja, dann krieg ich ja wieder Druck, und dann geh ich immer hin und sage: „Ich möchte mein Todesurteil.“ - Interviewerin: Und das heißt?

P1: Naja, er kann mich ja wieder fertig machen, ne. (lacht) (P1:545)

Hier zeigen sich die großen emotionalen Auswirkungen der in der Behandlung alltäglichen Besprechung der Blutzuckerwerte. Neben den Laborwerten sind Gewicht und Aussehen zentral. Viele der Interviewten erzählen, wie Gespräche über Körpermaße und Diäten ihre Begegnungen mit medizinischem Personal beherrschen und fast schon an Verhandlungen erinnern. Einige fühlen sich auf ihr Übergewicht reduziert und kritisieren, dass nicht ihre Beschwerden oder die medikamentöse Einstellung, sondern ihr Übergewicht im Fokus der ärztlichen Betrachtung steht. Mehrere haben den Eindruck, auf den ersten Blick verurteilt zu werden. Beispielhaft möchte ich dieses drastische Zitat einer Interviewten wiedergeben, die eine sehr kränkende Situation folgendermaßen beschreibt:

Da [in einer Kur] hab ich jedes Mal in 3 Wochen 10 Kilo abgenommen. [...] Dann hab ich auch 'ne ganze Zeit das Gewicht gehalten, aber trotzdem immer wieder jeder Arzt, ob sie mich kannten oder nicht, ob die meine Vorgeschichte kannten: Sie müssen abnehmen. Und das Dollste was mir [passiert] ist, das war beim Orthopäden, [...] der kannte mich überhaupt nicht, und der ist rein gekommen, kein „Tach“, nichts gesagt, nur umgedreht und über die Schulter gesagt: „Waren Sie schon immer so fett?“ Und gerade dieses, dieses Wort „fett“, da bin ich so schnell von die Liege hochgekommen, das weiß ich bis heute noch nicht, wie (lacht), und da hab ich den madig gemacht, 
das haben die drei Räume weiter gehört. Hätte er gesagt, „Waren Sie schon immer so dick?”, wäre das für mich anders gewesen, aber, „Waren Sie schon immer so fett?” Nee. Gut, und jedes Mal. (P1:008)

In diesem Zitat wird erlebbar, wie sich P1 geradezu entmenschlicht fühlt: Der Arzt scheint ihr gegenüber nicht einmal basale Höflichkeitsformeln zu verwenden. Auch wenn sie sich dagegen lautstark zu Wehr setzt, ist sie in ihrer Würde stark angegriffen. Deutlich wird auch, welche feinen Unterschiede der Wortwahl interpretiert werden: Das Adjektiv „fett“ wird als wesentlich abwertender wahrgenommen als „dick“. Dieser offensichtlich emotional wichtigen Unterscheidung soll im Folgenden noch weiter nachgegangen werden. P1 weist darauf hin, dass sie zum Zeitpunkt des geschilderten Ereignisses erfolgreich abgenommen und ein niedrigeres Gewicht gehalten hat, diese Leistung für einen Fremden aber nicht sofort sichtbar ist. Sie beschreibt das Gefühl, dass medizinisches Personal sie also aufgrund ihres Äußeren vorverurteilt - scheinbar ohne sich für ihre individuellen Bedürfnisse und Bemühungen („Vorgeschichte“) zu interessieren. Die fallübergreifend immer wiederkehrende Kritik, dass der lebensweltliche Kontext bei der ärztlichen Bewertung von Verzicht außer Acht gelassen wird, wird in Kap. 5.4.4 weiter untersucht.

Die kränkende Erfahrung, die P1 schildert, weist eine weitere Besonderheit auf: Sie befindet sich auf der Liege, als der Arzt den Raum betritt und ohne Begrüßung „über die Schulter" hinweg - also gewissermaßen unerwartet - ihr Gewicht kommentiert. P1 gelingt es, sich „schnell von der Liege“ aufzurichten und sich verbal zu wehren, dennoch bleibt der Eindruck eines ungleichen Machtverhältnisses zurück, in dem die Patientin der Bemerkung des Arztes zunächst schutzlos (wie "gelähmt“) ausgesetzt ist. Von einem sehr ähnlichen Erlebnis erzählt P30:

Ich krieg es auch von verschiedenen Ärzten zu hören, so wie zum Beispiel einmal beim Orthopäden, wo ich war, der mich, äh-. Er ist von hinten rein gekommen, hat noch nicht einmal 'Tag' gesagt, und hat gesagt: "Sie haben aber eine schlechte Haltung. Und abnehmen müssten Sie auch." Wums. Da dachte ich auch: "Tolle Begrüßung!" Aber man ist erst mal wie gelähmt da, weil, weil, ähm, das darf nicht sein, eigentlich, ne. Das ist doch eigentlich erst mal uninteressant. (P30:381)

Eine vergleichbare Erfahrung nennt P2:

Ich kenn also die ganzen Beschimpfungen, da gibt's also schon Mediziner, die das recht drastisch machen, die vor allem, wenn Sie da liegen irgendwo und sich nicht bewegen können, also sich nicht rühren können, [sagen:] „Also fünfzehn Kilo sollten wir erstmal abnehmen, nech, vorher komm ich nicht wieder“. Na gut, gut, kommt er nicht wieder. (P2:055)

Auch andere Interviewte gewinnen bereits beim ersten Zusammentreffen mit der me- 
dizinischen Welt aufgrund von äußeren Unterschieden den Eindruck, dass ihr Gegenüber unmöglich den gleichen Erfahrungsraum teilen und daher kein Verständnis für die Gewichtsprobleme haben kann. Das starke Gefühl, wegen des Aussehens persönlich abgelehnt zu werden (in diesem Fall von einer Diätberaterin) und nicht die zu erwartende Hilfe zu erhalten, findet sich in folgendem Zitat:

Wenn so 'n auch wieder dicker Mensch vielleicht hinkommt und der wird schon gleich mal von einer superschönen schlanken Frau [beraten], ein bisschen so hat man das Gefühl, wie guckt die mich denn gerade an, der, ne, der ihr Typ bin ich wohl nicht, aber dann ist die Frau da verkehrt [...] denn mir soll ja geholfen werden. (P3:191-192)

Die Erwartung in das medizinische Personal wird enttäuscht: Statt einfühlsame Menschen anzutreffen, die körperliches Leiden lindern und seelischen Beistand und Mitleid schenken, fühlen sich einige Interviewte geradezu diskriminiert. P3 äußert die Vermutung, benachteiligt zu werden, weil er nicht „der Typ“ der Diätberaterin ist. Hier zeigt sich, wie subtilste Kommunikation, wie ein Blick, eine Geste oder ein Wort die Beziehung zwischen Kranken und medizinischem Personal beeinflussen kann. Dabei sei darauf hingewiesen, dass die Interviews ausschließlich die subjektive und möglicherweise durch schlechte Erfahrungen antizipierte Wahrnehmung der Interviewten wiedergeben. P3 hat die Behandlung durch die Diätberaterin als abfällig empfunden, wozu neben ihrem tatsächlichen Verhalten möglicherweise viele andere Faktoren beigetragen haben. Auf einer so persönlichen Ebene argumentiert allerdings auch P1. Wenn sie kritisiert, aufgrund eines ersten äußeren Blicks verurteilt zu werden, scheint sie ihrerseits ebenfalls von den Körpermaßen der Ärztinnen und Ärzte Vorannahmen über deren Einstellung zu ihr abzuleiten: Sie hat die Kategorisierung in normal- und übergewichtige Menschen so verinnerlicht, dass sie von der Wahrnehmung, nur von schlanken Personen behandelt zu werden, verunsichert ist. In der Annahme, dass diese sie nicht verstehen und ablehnen könnten, sieht sie sich gezwungen, um mehr Empathie zu bitten. Ein Versuch besteht darin, die Verhandlung über das Körpergewicht aus der professionellen Arzt-Patient-Beziehung heraus - zumindest fiktiv - in den privaten Kontext familiärer Beziehungen zu übertragen:

Und wie gesagt, immer nur das Gewicht, das Gewicht, das Gewicht. Wenn man dann Ärzte hat, die nur soo sind ((Geste "schlank")). Den einen Tag hatte ich schon auf der Zunge, ich wollt schon fragen, was sagt ihr denn, wenn eure Frau mal zunimmt? Was macht ihr mit der, wollt, schickt ihr die in die Wüste? (P1:196)

Aus diesen Beispielen lässt sich eine zentrale Hypothese ableiten: Nicht nur beim ärztlichen Kontrolltermin, auch im Alltag erleben viele Interviewte immer wieder, dass von 
der körperlichen Erscheinung von Menschen auf ihr Verhalten und ihre Persönlichkeit geschlossen wird. Die Wahrnehmung des eigenen Körpers und das Selbstbild entwickeln sich im Vergleich mit anderen Menschen, welche indirekt gesellschaftliche Körpernormen widerspiegeln und reproduzieren. Auffällig ist, dass eine stabile Einteilung in schlanke und übergewichtige Menschen vorgenommen wird. Diese Hypothese erhärtet sich durch weitere Interviewaussagen. Diskret und humorvoll fragt sich z.B. P20 „wie [...] man als Moppelmensch“ abnimmt (P20:41). P3 ordnet sich den Menschen zu, die „verdammt [sind], Gerne-Esser, Alles-Esser zu sein“ (P3:148), und deutet an, dass eine strenge Zuordnung zu „den Dicken“ oder „den Dünnen“ bereits in der Kindheit erfolgt und dann konstant bleibt:

Wie gesagt, ich bin schon immer dick, ja, schon als Kind, ja, und natürlich hat man das gemacht und auch vielleicht mehr gemacht wie andere Kinder, die nicht so dick waren [...]. Der eine hatte ein Glas Cola und ich hab vielleicht zwei oder drei getrunken, ne, nicht, oder eine Portion Pommes und ich hab 'ne große, gro-, Po-, Pommes gegessen, ne, und so wurde das immer mehr und so ging die Schere immer mehr auseinander. (P3:151-152)

In der Erzählung von P3 wird auch die Ambivalenz der Selbstwahrnehmung und Reflexion deutlich. Während er sich im obigen Zitat die Verantwortlichkeit für die Gewichtszunahme zuschreibt („vielleicht mehr [...] [als] andere“), normalisiert er an einer anderen Stelle das Übergewicht fast schon im Sinne eines Schutzschildes: „da heute jeder, weiß ich nicht, [jeder] wievielte Deutsche zu dick ist, ne, jeder zweite mittlerweile schon, aber jeder dritte doch bestimmt" (P3:151). Wenn dagegen P30 verzweifelt darüber klagt, dass es ihr trotz größter Anstrengung nicht gelingt abzunehmen, kann sie auf einen solchen Schutzschild nicht zurückgreifen und scheint ganz allein mit ihren Problemen dazustehen:

Bei anderen klappt das wunderbar, dass sie eben halt dann abnehmen. Bloß bei mir klappt das nicht. Ich weiß auch nicht, warum, wieso, weshalb. Keine Ahnung. Es ist eben halt so. Es ist, ähm, war zwar früher nie der Fall gewesen, dass ich so eben halt auseinander gegangen bin. Aber seit ein paar Jahren ist, ist es eben halt der Fall.

Und ich kämpfe ja dagegen an. Ist ja nicht so, dass ich mich nur hier hinsetze und alles in mich hineinschaufele. Nein. Ich, ich achte da drauf. Ich verzichte. Und das, es tut schon manchmal richtig weh, weil sogar, wenn andere da in sich hineinstopfen, und Du sitzt da mit Deinem Tellerchen und isst das, und-. Ah, das frustriert. Da, da, da, und dann sagt man sich selber auch: "Hey, was soll ich denn noch alles machen? Was? Setzt ihr euch mal dahin und habt das, was ich habe. Und dann können wir weiterreden". (P30:385)

In diesem Zitat werden mehrere Dimensionen von Verzicht deutlich. Zuerst beklagt die Interviewte die Ungerechtigkeit, selbst mit den größten Bemühungen nicht abnehmen zu können, während dies Anderen scheinbar mühelos („wunderbar“) gelingt. Sie zeigt sich darüber fassungslos und wehrt so jeden hypothetischen Vorwurf ab, sich falsch verhalten zu haben („Es ist halt eben so“). Im Gegensatz zu P3 stellt P30 klar, nicht 
immer dick gewesen zu sein, sondern erst „seit ein paar Jahren auseinandergegangen zu sein“. Das Wort „auseinandergehen“ kann auf mindestens zwei verschiedene Arten gelesen werden: P30 könnte damit äußern, dass sie das Zunehmen als einen unkontrollierbaren Prozess wahrnimmt, der eher mit körperlichen Eigenschaften, z.B. Alter oder schlaffem Bindegewebe assoziiert ist als mit übermäßiger Nahrungsaufnahme. „Auseinandergehen“ kann aber auch im Sinne einer Fremdzuschreibung mit dem Vorwurf einhergehen, sich nicht „zusammengenommen“ zu haben. Dieses Zitat zeigt, wie groß der Druck ist, die eigenen Verzichtsbemühungen darzustellen, und wie frustrierend es ist, wenn diese nicht sichtbar sind. In zweiten Teil des Zitats zeigt sich - auch sprachlich mit den Ausdrücken „,hineinstopfen“, „Tellerchen“ und dem dreifachen „Da, da, da“ - der große Druck, der mit der Forderung, sich zu beherrschen, auf P30 lastet. Die Interviewte fühlt sich gezwungen, ihr Verhalten gerade im Kontrast zu anderen als moralisch einwandfrei darzustellen. Während andere ungerechterweise Speisen „in sich hineinstopfen“ (dürfen), setzt sie fast zwanghaft dagegen, dass sie nicht auf unzivilisierte Weise Essen in sich „hineinschaufelt“, ja nur ein „Tellerchen“ benutzt und leidet („es tut schon manchmal richtig weh“). Man spürt geradezu ihre Angst, dass andere ${ }^{17}$ aufgrund ihres Äußeren falsche Rückschlüsse auf ihr Essverhalten ziehen und sie deshalb moralisch verurteilen könnten. Außerdem fühlt sich P30 benachteiligt und von sozialen Zusammenhängen ausgeschlossen (vgl. Kap. 5.4.2). Sie fühlt sich von anderen Menschen unverstanden, die nicht die gleichen Erfahrungen mit erfolglosen Diät-Versuchen haben. Dieser Eindruck findet sich bei P20 wieder:

Und, äh, die Leute, die einem immer erzählen, äh, wie man das alles am besten auf die Kette kriegt, das sind meistens die, die da keine Probleme mit haben. [...] Das sind genau die gleichen, die auf irgendeiner Feier kommen, selber Kleidergröße 38 haben, und fangen dann über Diät an zu reden, und sprechen dann davon, äh, wie man denn, ne, am besten, und wie furchtbar dick sie sind, und was sie doch alle für Probleme haben. Dann könnte ich so meine Sachen nehmen, aufstehen und gehen, weil ich das also-. Das ist für mich sowieso kein Gesprächsthema. Aber das ist auch wahrscheinlich, das sind meistens auch die, die irgendwie geistig unterentwickelt sind. Aber ist so. (P20:397-401)

Hier wird deutlich, welche Rolle die Bewertung von Körpermaßen in alläglichen sozialen Beziehungen spielt und wie P20 sich am liebsten dem Thema entziehen möchte. Die Nähe zum Schönheitsideal wird anhand bestimmter Kleidergrößen gemessen. Der

\footnotetext{
17 Wie sich ein paar Sätze vor dem ausgewählten Ausschnitt zeigt, adressiert P30 ihre Erzählung in diesem Zitat an das medizinische Personal, von dem sie sich immer wieder gedrängt fühlt, abzunehmen. Die aufwendig formulierte Erklärung für die fehlende Gewichtsabnahme ist jedoch ein Hinweis dafür, dass sie moralische Diskreditierung längst nicht nur vonseiten der Ärztinnen und Ärzte fürchtet, sondern von ihrem privaten alltäglichen Umfeld und möglicherweise auch von der Interviewerin.
} 
Wunsch, sich daran anzupassen, wird als gesellschaftlicher Zwang karikiert, dem, wie P20 in derber Weise ausdrückt, intelligente Menschen keine Folge leisten dürtten. Sie zieht klare Grenzen zwischen den Schlanken, die es nur vermeintlich schwer haben, und denen, die wirkliche (Gewichts-)Probleme haben. Zwar stellt sie sich als über Banalitäten, wie einem Schönheitsideal nachzujagen, erhaben dar, aber ihre drastische Wortwahl zeigt, wie sehr sie sich durch die Koketterie anderer angegriffen fühlt. Die vernünftige Überzeugung, dass Äußerlichkeiten „kein Gesprächsthema“ sind, sperrt sich der Realität und auf der Erzählerin lastet ein hoher Druck, dem Schönheitsideal zu genügen.

Die Angst vor charakterlicher Verurteilung bei schlechten Laborwerten und Übergewicht zeigt sich meistens indirekt in den Erzählungen, z.B. in dem Bemühen, sich selbst als besonders genügsamen Mensch darzustellen. P30 spricht den gefürchteten impliziten Vorwurf, sich nicht genug um Verzicht zu bemühen, zumindest im Interview auch offen aus:

[Die Ärzte sagen:] "Machen Sie mehr Sport". [...] Und dann wird man weggeschoben, weil die eben halt sagen, ich bin nur zu faul um etwas wirklich so durchzuführen. Wenn ich jetzt aber den anderen erzähle, was ich so esse, damit mein Zucker runter geht, dann sagen die: "Hey, das wird ja immer weniger." Aber der Zucker geht immer höher. Ja. Das wollen die immer alle gar nicht glauben. (P30:067)

Sie deutet an, dass sie vom medizinischen Personal als „faul“ charakterisiert wird. Dagegen lässt sie in ihrer Erzählung „andere“, vermutlich Bekannte oder Freunde wie „Zeugen“ zu Wort kommen. Diese bestätigen, dass sie „immer weniger“ isst, also entgegen der wahrgenommenen ärztlichen Meinung - fleißig verzichtet.

Im folgenden Kapitel werden Versuche der Interviewten, sich vor moralischer Verurteilung zu schützen, genauer dargestellt: Auffällig sind zum einem relativ abstrakte Abgrenzungsversuche von „den Maßlosen“. Zugleich bemühen die Interviewten komplexe Erklärungsmuster zu den Ursachen von Diabetes und Übergewicht, besonders mit Blick auf die mögliche eigene Schuld.

\subsubsection{Sich von „den Maßlosen“ abgrenzen - (un)schuldig sein}

Die in der medizinischen Welt wie im sozialen Alltag gleichermaßen dominante Einteilung in normal- und übergewichtige Menschen bedrängt viele Interviewte. Sie legen großen Wert auf individuelle Beschreibungen, wie bereits anhand der subtilen, fast schon kleinlich wirkenden, Unterscheidung von „fett" und „dick“ deutlich wurde (vgl. 
Kap. 5.5.1). Auch P15 „kämpft“ um begriffliche Feinheiten:

Aber, ähm, ich war immer pummelig [...]. Aber es ist auch nie so gewesen, dass ich, äh, sagen kann, so, so unförmig, äh, dick. Das gibt es ja auch, ne. Das, das nicht. (P15:0540)

Überzeugt, dass nicht ihr eigenes Verhalten Ursache ihres Übergewichts ist, verweigert sich P1 einer Zuordnung zur Gruppe „der Maßlosen“ und zieht eine feine moralische Grenze:

„Sie müssen abnehmen!“ - das war dann wieder das Nächste. Und auf diesen Satz, da reitet er [gemeint ist der Arzt] bei mir rum, ich weiß nicht, was er den anderen Frauen sagt, die wirklich soo (breitet Hände aus) sind. Nur er darf dick nicht mit dick vergleichen. Ich bin dick nicht durch 's Essen. Es gibt aber auch Dicke, die sind dick durch's Essen. (3) $\mathrm{Ne}$, das ist ' $n$ Unterschied. (P1:521)

Selbst Menschen, die - auch medizinisch betrachtet - nicht abnehmen müssen, fühlen sich offensichtlich durch die Forderung nach Verzicht und Disziplin angesprochen und zu entsprechend „gesundem Verhalten“ gedrängt:

Ja nur wie gesagt, also, das Laufen- [...] Ja, hab habe ich also jetzt nicht, wegen des Diabetes, äh, Erkrankung gemacht, sondern [ich] war ein auch ein sportlicher Typ. (P21:496)

P21 ordnet sich hier ganz klar einem „sportlichen Typ“ zu und grenzt sich von übergewichtigen Menschen, die viel eher dem „Diabetes-Typ“ entsprechen, scharf ab („Kolleginnen und Freundinnen die beleibter sind und, eh, haben nichts und ich als schlanke Person, sag ich mal, haben da diese hohen Werte, ja?“ [P21:622]; vgl. Kap. 5.4.2, S. 53). Während sie es als Ungerechtigkeit empfindet, trotz ihrer Schlankheit an Diabetes erkrankt zu sein, ist es gerade diese Abgrenzung von den anderen, welche P20 motiviert, weiterhin konsequent zu verzichten.

Ja und wie gesagt, die Patienten, die dort waren, waren ja alle, also (2) dick, beleibt und ((lachend)) also übergewichtig, und da dacht ich schon immer: „Na, damit hast du nun nicht zu tun. Und mach weiter so.“ (P21:602)

P21 sagt, ihr „fällt es nicht schwer“ (P21:534), sich an die Diät zu halten. (Interessant ist allerdings, dass sie im Rest des Interviews betont, wie „hart“ ihr die Einschränkung erscheint). Mit weiteren Aussagen wie „ich (...) war auch nicht der Typ, der 'ne halbe Tafel am Abend aufessen muss“ (P21:534) und „Torte nicht zu essen, fällt mir sowieso nicht schwer. War nie mein Ding“ (P21:534), grenzt sich P21, prophylaktisch könnte man sagen, gegen den hypothetischen Vorwurf der Maßlosigkeit ab. Deutlich wird der argumentative Aufwand, mit dem sie sich zum Thema Verzichten positioniert, der den bereits beschriebenen Rechtfertigungen von P30 nicht unähnlich ist („wenn andere da in sich hineinstopfen, und Du sitzt da mit Deinem Tellerchen." [P30:385]; vgl. Kap. 
5.5.1., S. 65). Die Interviewte P27 kontrastiert ihr Verhalten mit dem einer übergewichtigen Arbeitskollegin, die sich ungesund ernährt, während sie selbst kalorienarme Kost wählt. Vor dem Hintergrund dieser Erzählung spricht sie auch einen inneren Kampf an, bei dem sie einer Süßigkeit nicht widerstehen konnte. Warum der Verzehr der Schokolade nicht maßlos war, erklärt sie aufwendig: erstens sind die Tafeln "hautdünn“, was eine geringe Menge suggeriert. Zweitens hat sie nicht auf einmal eine ganze Tafel gegessen, sondern „einen ganzen Tag über“. Schließlich lässt sie ihren Partner in der Erzählung zu Wort kommen, der wie ein „Zeuge“ (vgl. P30, Kap. 5.5.1, S. 67) bestätigt, dass dieses „ungesunde Verhalten“ eine Ausnahme war.

Ich habe mir denn immer ein Fischbrötchen mit sauren Hering geholt. Da habe ich gesehen, dass das nicht viele Kalorien hat und sie [meine Arbeitskollegin] hat immer große Stange mit Butter und Schinken und Gurke und das alles geholt und gegessen. [...] Die hat aber keine Zucker. [...] Die war so breit. [...] Die hat diesen Jojo-Effekt, ne? Dann isst sie mal nicht und dann isst sie heimlich und isst sie Süß-Süßes.

Bin ich gar nicht für! Mal, aber ich habe schon einmal eine Tafel Schokolade aufgegessen, mit Chili, die wollte ich probieren. Diese ist, die sind ja haut-dünn, ne? [...] Vier, fünf so Riegel einen ganzen Tag über. [...] [Dann habe] ich zu meinem Mann gesagt: „Weißt du was, ich habe die Tafel ganze Schokolade aufgegessen." Da hat er gesagt: „Weißt du was, das hast du auch mal gebraucht.“ Weil ich ansonsten nie-. Mein Mann, der isst ja Süßes. Och, der kann ja Süßes essen, Kuchen und das alles. (P27:110)

Aus Angst, für Körpermaße oder diabetische Stoffwechsellage schuldig gesprochen zu werden, entwickeln viele der Interviewten einen Anspruch, Charakterstärke und das „richtige“ Verhalten unter Beweis zu stellen. Diesem treten einige mit der Vermutung gegenüber, ihr Körper funktioniere nicht nach normalen, beherrschbaren Regeln - und Verzichten nütze daher nichts bzw. ihre Körpermaße und Laborwerte spiegelten nicht ihren Verzicht wider. In einer Selbstbeschreibung von P20 findet sich die Kleidergröße als Maßstab für "gute“ Eigenschaften wieder (vgl. Kap.5.5.1, S. 67):

Und habe jetzt festgestellt, dass mir so morgens diese, sagen wir mal, viertel bis halbe Stunde [Zeit für mich nehme] - viel länger ist es nicht, weil ich dann, schon wieder kribbelt. Normalerweise müsste ich eigentlich Größe 38 haben. Aber, ähm, [dass] das schon wieder kribbelt, dass ich denke: "Du könntest das noch machen, und das noch tun." (P20:156)

P20 beschreibt sich als rastlos und aktiv, d.h. auch fleißig, und hebt hervor, dass sie aufgrund dieser Eigenschaften eigentlich erwartet, schlanker zu sein. Sie erlebt ihren Körper als nicht durch ihr Verhalten steuerbar. Während sie sich durch diese Aussage gegen den unausgesprochenen Vorwurf wehrt, dass sie „faul“ wäre und deshalb übergewichtig sei, beteiligt sie sich implizit an der moralischen Abwertung des Dickseins bzw. des dicken Menschen.

P1 wehrt sich gegen den Vorwurf des Arztes, nicht genügend verzichtet zu haben, mit 
der Begründung, in ihrem Körper sei seit ihrer Lungenembolie „alles anders als [...] beim normalen Menschen“. Die Erfahrung, dass sie trotz Verzichts manchmal ab- und manchmal zunimmt, veranschaulicht sie durch das Bild eines Schalters, der sich willkürlich umstellt:

Und der erste Satz war ja wie gesagt immer: „Sie müssen abnehmen.“ Und dann dies nicht essen, das nicht essen. Und, hab ich doch eigentlich auch gar nicht. Ich hab mich ja dran gehalten, was er gesagt hat. Aber ich meine, ich sage ja immer: bei mir ist ja nix normal im Körper. Durch diesen Knall damals, da ist wirklich alles, (holt Luft) alles anders als wie beim normalen Menschen. (2) Da hab ich manchmal das Gefühl, als wenn ich ' $n$ Schalter im Körper habe, der schaltet sich um, und wenn ich heute von dem gleichen Kram abgenommen habe, kann es sein, dass ich morgen mit dem gleichen Essen wieder zunehme (hebt die Hände und lässt sie auf den Tisch fallen) und keiner weiß, woher warum wieso weshalb. (P1:120)

Damit stellt sie sich - für ihre eigene Person im Sinne einer Ausnahme - gegen die scheinbar gesellschaftlich vorherrschende Annahme, Übergewicht sei etwas selbst Gewähltes oder Ausdruck von Willensschwäche.

Eine Variante wäre, die Schuld übergewichtig oder an Diabetes erkrankt zu sein, in der - ebenfalls nicht selbst steuerbaren - Genetik zu suchen. P30 vermutet, dass das Fehlschlagen ihrer Präventionsversuche in ihren Genen begründet ist, die für die Funktionseinschränkung ihres Körpers verantwortlich seien:

Aber bei mir hat es irgendwie nicht hingehauen. Bei mir ist das diese Veranlagung, die ich mal sage, die ich habe, wo eben halt die Bauchspeicheldrüse doch nicht mehr so will, wie sie sein, äh, sein sollte in diesem Alter. (P30:129)

Ganz ähnlich erklärt sich P15 ihr Übergewicht:

Ich war immer die Dicke. [...] das zog sich wie so ein roter Faden durch mein Leben. Denn, ähm, meine Schwester zum Beispiel, die ist irgendwie anders, anders veranlagt. Die war immer schlanker und- (4s). Ja, aber es ist leider so. Ich war immer so wie meine Mutter. Meine Mutter war auch immer pummelig und-. ((räuspert sich)) So eine gewisse Veranlagung scheint das zu sein. Ich muss mich ja jetzt auch mit irgendetwas trösten ((lacht)). (P15:0539)

Neben diesen mit Genetik argumentierenden Konzepten zur Krankheitsursache gibt es andere, bei denen gesellschaftliche Marginalisierung übergewichtiger Menschen als entscheidender Faktor gesehen wird. Einer der Interviewten macht in der feinsinnigen Beschreibung der gesellschaftlich unterschiedlichen Behandlung von Magersucht und Adipositas deutlich, wie er durch die moralische Wertung von Körpermaßen in der Gesellschaft betroffen ist und darunter leidet oder sich gar zu irrationalem Verhalten gedrängt fühlt.

Das ist aber auch 'ne Krankheit, denk ich, dieses Zu-dick-sein, ja. Das hat man ja jahrelang einfach nur, äh, so hingenommen und hat eventuell die dicken Leutchen noch bestänkert, provoziert, aufgezogen, hin und her, dass das eventuell aber auch 'ne Krankheit ist-. Komisch, über diese Magersucht, da regt sich keiner drüber auf, die, die, die Mädchen oder Jungen, ja, Jungen gibt es da ja auch, die werden nicht gehänselt. 
$\mathrm{Ne}$, aber die Dicken, ne, auch nicht gut so und dann, was machen die dann? [Die] ärgern sich, gehen in die nächste Frittenbude und hauen sich noch was rein, die Dicken, so machen die das, nech, und Endergebnis Diabetes. (P3:154)

Im zweiten Abschnitt stellt P3 überspitzt die Wahrnehmung der Außenwelt dar: „Die Dicken“ gehen in die Frittenbude und "fressen sich den Diabetes an“. Dabei betont er die seiner Meinung nach vorgeschalteten Mechanismen: Ärger und Frust führen zum Verzehr der ungesunden Lebensmittel. In dieser Argumentation, dass nämlich die Gesellschaft durch Ausgrenzung das gesundheitsschädliche Verhalten fördere, wird deutlich, wie wichtig P3 eine Verteilung der "Schuld“ an der Verursachung der Krankheit ist, die bisweilen allein auf den einzelnen Menschen mit Diabetes projiziert zu werden scheint.

Ein ähnliches Muster lässt sich bei P1 erkennen. Auf die Bitte der Interviewerin das oben zitierte Bild des „Schalters“ (vgl. Kap. 5.5.2, S. 70) näher auszuführen, begründet P1 die zuvor von ihr durch körperliche Dysfunktion erklärte Gewichtszunahme mit konkreten emotionalen Reaktionen auf den Tadel des Hausarztes.

Ja, das war dann, wo mich der Hausarzt mal so richtig runtergeputzt hat, weil ich ja wieder schön zugenommen hatte. Und da hab ich gedacht: das darf nicht wahr sein. Und dann bin ich nach Hause gekommen und dann hab ich richtig gemerkt, wie der Schalter sich umlegte: So, heute ((lässt Hand auf den Tisch fallen)) isst du Mettwurstbrot ((lässt wieder Hand auf den Tisch fallen)) und du isst dies und du isst das. Was ich vorher gar nicht gegessen habe. Und dann brauch es wieder 'ne gewisse Zeit, bis es wieder „klick“ macht und dann geht's wieder runter. (P1:124)

Sie widerspricht dem Bild im vorhergehenden Zitat: erst ist es ein „Schalter“, der bewirkt, dass sie von dem gleichen Essen einmal zu- und einmal abnimmt. Jetzt steht der „Schalter" dagegen für die negativen Erlebnisse bei der ärztlichen Kontrolle, die in ihr eine so starke Gefühlsreaktion hervorrufen, dass sie ihre Selbstkontrolle verliert. Die als völlig einseitig erlebte Schuldzuweisung durch die Gesellschaft wird nun umgekehrt zu einer Schuld der Außenwelt: deren Kränkungen sind die wahren Gründe für „Frustessen“. Die betroffene Person scheint dann dem Verlauf der Heißhungerattacke nur noch zusehen zu können. Auch dieses Verhalten wird zu einem häufig vorkommenden Phänomen normalisiert:

Interviewerin: Mh, und dann hatten Sie noch erzählt, mit dem, mit dem Frustessen, dass man nach solchen Situationen...

P1: Ja, da bin ich nicht die Einzige! (P1:318-320)

Es gibt auch Interviewte, die sich die Verursachung der Krankheit selbst zuschreiben und "Schuldeingeständnisse" machen: 
Wenn das also dazu beitragen sollte, dass ich also wirklich wieder auf Normalwerte komme, dann mach ich das und da hab ich dann also innerhalb von neun Monaten 40 Kilo abgespeckt. [...] Diese ungesunde Lebensweise [ist es] gewesen, die das Gewicht hochgetrieben hat, äh, und die auch im Endeffekt, äh, wenn ich das also heute so sehe, ähm, die Diabetes verursacht hat, ne. (P16:0589-1052)

Für P16 scheint das Schuldeingeständnis und die Überzeugung bzw. die Motivation, selbst etwas „beitragen“ zu können, verknüpft zu sein. Bei anderen ist dies nicht so eindeutig.

Es ist ja auch nicht in dem Sinne, ich sage immer, es ist ja keine Krankheit. Des, es ist ja 'n Selbstverschulden. Es, Diabetes 1, gut, aber Diabetes 2- Hätte ich mit achtzehn Jahren so weiter gemacht, dann würde ich heute wahrscheinlich noch Marathon laufen oder irgend so was. Obwohl, wenn man so liest, die haben auch immer alle sehr viel Diabetes. Warum auch immer. Aber, äh, man, man, dann wär vielleicht diese ganze Situation gar nicht so dazu gekommen. (P6:376)

Obwohl P6 hier sogar so weit geht, die Ursachen des Diabetes Typ 2 ganz seiner eigenen Verantwortung zuzuschreiben, ist dieses „Schuldeingeständnis“ nicht ganz ohne Zweifel: Er äußert Erstaunen darüber, dass auch Menschen, die Marathon laufen, Diabetes haben. Möglicherweise ist dies eine vorsichtige Frage danach, ob nicht doch mehr Faktoren bei der Krankheitsentstehung mitwirken.

P27 betont ebenfalls inr Wissen um die „Selbstverschuldung“:

Ich weiß, das fällt ja alles einem schwer, wenn der Bauch so dick ist. Das Bücken fällt einem schwer, so 'ne? Man weiß man halt selber Schuld. Man hat selber Schuld. (3) Was soll man da machen? Man muss so eisern sein, vielleicht fin-finde ich noch mal den Sprung. So langsam. Oder auch nicht. Das kann ich nicht sagen. Das werde ich auch nicht versprechen. (P27:110)

Interessanterweise führt die Einsicht bzw. die Erklärung, die Krankheit bzw. das Übergewicht verursacht zu haben, nicht automatisch zum Verständnis, die Situation selbst ändern zu können („Was soll man da machen? Man muss so eisern sein, vielleicht [...] finde ich den Sprung. [...] Das kann ich nicht sagen.").

Genauso muss die Vorstellung eines kaum von außen bzw. durch den Willen beeinflussbaren Körpers nicht unbedingt heißen, einer Krankheit wie Diabetes nichts entgegensetzen zu können. Bei P32 stellt hier eine religiös gefärbte Sicht diesen Zusammenhang - und damit auch das Thema Verzicht - komplexer dar: An einer Stelle beschreibt er den Körper - mit der Fähigkeit, ihn auflaufen zu lassen - fast als Gegenspieler des Bewusstseins. Der Körper hat inm die „Rechnung“ für sein Verhaltens gegeben, wird als „ehrliches“ Abbild des Lebensstils oder „Gewissen“ verstanden.

Der Körper hat gesagt: "Gut, mach weiter." Und dann Endeffekt habe ich dann die Rechnung gekriegt. Das war für mich, da, weil ich nichts gemacht habe. (P32:382) 
Dennoch bildet der autonome kranke Körper die letzte Instanz: die Grenze seines Handlungsspielraumes, die P32 akzeptieren muss. Eine solche Sicht deutet sich im nächsten Zitat von P32 an:

Interviewer: Und das war, wenn ich doch nochmal nachfragen darf, das war dann aber auch die Zeit, wo Sie plötzlich nicht mehr so dünn-.

P32: Das war, doch, doch, äh, Anfangszeit bis, äh, '75, '76, das war alles noch. Ach so, das habe ich vergessen. Entschuldigung. Äh, '75, bis dahin war alles, also, war ich so. Und dann kam einfach dann die Zeit, wo dann einfach das, der Gewicht, der Übergewicht-. Warum, weshalb? Das weiß ich nicht. Weiß ich nicht. Vielleicht hätte man damals vielleicht, äh, aber das werde ich auch nie erfahren. Das ist, das ist, das ist Schicksal ((türkisches Wort)), das muss ich so hinnehmen. (P32:545-561)

P32 weiß die plötzliche Gewichtszunahme im Kindesalter nicht zu erklären, zweifelt daran, ob „damals vielleicht“ etwas hätte geändert werden können und verweist abschließend auf das Schicksal, das hinzunehmen ist. Dieses Konzept ermöglicht ihm, die Gegebenheiten zu akzeptieren, hindert ihn jedoch nicht daran, jetzt etwas dagegen zu tun (P32 erzählt an anderer Stelle von einer erfolgreichen Gewichtsabnahme, die dazu geführt hat, dass seine Medikation von Insulin auf Tabletten umgestellt werden konnte). Auch wenn sein Übergewicht damals durch „Kräfte von oben“ verursacht und also unvermeidbar war, ist sein jetziges Handeln nicht vorherbestimmt, sein Schicksal besteht womöglich darin, die gegebene Situation meistern zu müssen. Diese Sichtweise schützt P32 aber davor, die vollständige Verantwortung für seinen Körper übernehmen zu müssen.

\subsubsection{Körpermaße normieren, akzeptieren, verändern}

Viele der Interviewten beschäftigen sich auf unterschiedliche Weise mit dem Thema „Radikaldiäten“. ${ }^{18}$ Angesichts der bereits erarbeiteten Erfolgskriterien von Verzicht und der übermächtigen Körpernormierung und Diskriminierung von Übergewichtigen, scheint eine starke Gewichtsabnahme einen Übertritt zur Gruppe „der Dünnen“ und damit gesellschaftliche Akzeptanz zu ermöglichen. Beim Nachgehen dieser Vermutung stellt sich das Thema komplexer dar: Das Streben nach Akzeptanz des eigenen Körpers lässt manche eine radikale Veränderung fast als verwerflich erscheinen.

Man sollte auch nie herkommen und sagen: "Ich muss jetzt zwanzig Kilo abnehmen." Das ist, das sieht so unglaublich viel aus, dass man Angst hat. Man sollte sich mal vorstellen - ich habe das ja auch immer überall dann gelesen. Und wenn ich hier manchmal in den Zeitungen oder beim Frisör oder keine Ahnung, wo man dann mal liest. Hier,

18 Der Begriff Radikaldiät ist hier nicht im Sinne von „Null-Diät“, also temporärer Nahrungskarenz zu verstehen, sondern als radikale Veränderung des äußeren Erscheinungsbildes, durch starke Gewichtsabnahme. 
dann zeigen sie ihre Hosen, die sie vorher anhatten und dann später. Und also finde ich alles völlig Banane. [...] Völlig bescheuert ist, wenn man anfängt und jetzt wild-: "Ich esse jetzt gar nichts mehr." (P20:262-267)

Durch Verzicht abnehmen bei Diabetes, ist mit einer Positionierung zu Körpernormen verbunden. „Gerade als Frau“, betont P20 immer wieder, geht es um die Verhandlung von Schönheitsidealen, z.B. wenn sie sich mit anderen Frauen unter der Dusche im Schwimmbad vergleicht.

Das ist vielleicht jetzt auch noch so eine Sache. Und das geht vielleicht mehr die Frauen als die Männer an. Die sehen das ja vielleicht etwas lockerer. Wenn wir da [...] unserem Duschraum stehen, dann ist es für uns Frauen einfach selbstverständlich, dass wir unseren Badeanzug ausziehen und uns abduschen. Das muss man lernen. Äh, auch das ist ein Problem für die, die etwas mehr Gewicht haben als die anderen.

Natürlich ist es toll, wenn da jemand reinkommt, der schlank ist, und steht da unter der Dusche. Aber so bin ich nun mal nicht. Und wenn, ich sage mal, dann, dann muss man einfach lernen, dass man auch sagt: "Ich bin eben wie ich bin. Und jetzt ziehe ich den Badeanzug aus." Das ist auch so eine Überwindungsgeschichte. "Und dusche mich hier jetzt ab und zicke da jetzt nicht rum." Das bringt mir ja gar nichts.

Und die anderen, die gucken jetzt, oder was. Ich sage immer: "Die gucken zweimal. Einmal hin, und einmal wieder weg." [...] Man sollte nicht denken: "Boah, jetzt bin ich dick“. Und wenn mich jetzt einer sieht und die gucken nur mich an, und die lachen mich womöglich insgeheim aus. Wenn die hier raus gehen, dann sagen sie: „Hast Du die schon mal gesehen“, oder so. Ähm, das finde ich sowieso oberungezogen. So was tut man einfach nicht. Das ist oberungezogen.

Ähm, wir können uns alle nicht malen. Wir sehen alle im Grunde genommen gleich aus, nur eben die Variationen sind anders. Und ich denke einfach mal, das kommt nicht darauf, was das ist, sondern es kommt darauf an, was da ist und was da ist [zeigt auf Herz und Kopf]. Das ist das Wichtigste. Und wenn das passt, dann ist das [Aussehen], wenn es dann eben nicht mehr ganz so schön ist, okay, ne. (P20:338-342)

Eine scheinbar banale Situation, wie das Duschen nach dem Frühschwimmen, kann also einen belastenden, widerstreitenden inneren Dialog auslösen: Es wird - anderen bereits zitierten Interviewten ähnlich - eine scharfe Grenze zwischen denen, „die etwas mehr Gewicht haben“, und "den anderen“ gezogen. Indem P20 beschreibt, dass es eine „tolle" Wirkung hat, wenn jemand Schlankes hereinkommt, skizziert sie das gängige Schönheitsideal. Tapfer versucht sie sich aufzuwerten, indem sie es als Beweis von Stärke und Souveränität schildert, wenn sie sich überwindet, ihren Körper zu zeigen. Als Mittel der Kontrastierung werden scheinbar charakterschwächere Menschen, die „rumzicken“ und ihren Badeanzug nicht ausziehen, erwähnt. Ebenso wie von den zuvor beschriebenen schlanken Menschen, die kokett über ihre "Gewichtsprobleme" plaudern, fühlt sich P20 hier von denjenigen, die sie vermeintlich mit abschätzigen Blicken mustern, angegriffen und reagiert scharf darauf. Gegen die Angst, ausgelacht zu werden, versucht sie sich selbst stark zu machen, indem sie diese Menschen zu „oberungezogenen“, also nicht ernstzunehmenden Interaktionspartnern degradiert. Im 
Sinne des zumindest offiziell allgemein anerkannten Satzes „Die inneren Werte zählen“ verweist sie auf Verstand und Empathievermögen. Das gängige Schönheitsideal attackiert sie als unbrauchbares Maß, da „wir alle gleich aussehen, nur die Variationen sind anders“. Durch das erneut verwendete „wir“, bezieht sie die Zuhörenden mit ein und erhebt ihre Aussage damit zur „natürlichen“ Tatsache. Wenngleich P20 mutmaßt, dass Schönheitsnormen besonders unter Frauen eine Rolle spielen, ist die Geschichte, die P3 erzählt, ein Beispiel für die ähnlichen und nicht minder schmerzhaften Erfahrungen eines Mannes. Wieder geht es um Vergleich und Bewertung im Schwimmbad:

Ich bin gehänselt worden, selbstverständlich, ich bin ausgelacht worden, wenn ich im Schwimmbad gewesen bin. Ja, was mein Sie, warum ich heute nicht ins Schwimmbad gehe. [...] Sie haben das Gefühl, jeder Mensch, der guckt mir auf meine Männerbrüste, die ich ja logischerweise habe, wenn ich zu dick bin, ja, die haben mir das selber, selber haben die mich gestört, jeder [...] guckt da hin und jeder will gerade loslachen [...], auch wenn Sie dann den Pausenclown machen, was weiß ich und als erste vom 5-m-Brett springen ja, und so weiter und so fort. Aber trotzdem in, in, in, in, in, in mir selber, oder in dieser Person selber was da- [...] Die umspielen das, logisch, ne, indem sie, ja, Pausenclown machen, indem sie lustig sind, [...] das sind dann immer, immer angenehme Menschen, die lustigen [...], das sind immer die Dicken, [...]. (P3:167-168)

Wie sehr es sich beim Thema Übergewicht, selbst bei erfolgreichem Abnehmen, um ein schambelastetes Tabu handelt, wird in den folgenden Äußerungen von P20 deutlich. Immer schwingt darin die Furcht vor einer moralischen Diskreditierung mit - denn letztlich könnte die Außenwelt das Abnehmen wegen Diabetes als Beweis früheren Fehlverhaltens betrachten. P20 erzählt, wie sie einmal ein Lob für ihre erfolgreiche Gewichtsreduktion als eher unangenehm empfunden hat, weil die erhöhte Aufmerksamkeit anderer für ihre Körpermaße ihr nicht gefällt und sie selbst im Nachhinein noch eine Verurteilung fürchtet.

Und dieses, dass ich dann gesagt habe: Ich muss jetzt abnehmen, und dass dann irgendwann mal jemand gesagt hat: "Boah, hast Du abgenommen?" Und im ersten Moment ist einem das dann so- ja, man ist stolz, aber man will eigentlich gar nicht da so drauf angesprochen werden. Weil, ähm, ja, man müsste sich ja dann erklären, man müsste ja sagen: "Ich habe da jetzt Diabetes Typ 2 und ich muss jetzt abnehmen." Und dann kommt ja so eine Frage. "Wieso, wie viel wiegst Du denn?" Und es gibt ja Leute, die unglaublich impertinent fragen können. Da muss man sich ein bisschen ein dickeres Fell anschaffen, muss einfach sagen: "Das geht Dich gar nichts an", und peng. (P20:91-95)

Die körperliche Erscheinung ist unmittelbar mit dem Selbstbild verbunden. Eine radikale Gewichtsabnahme wäre für P20 ein fast gefährlicher Eingriff in ihre Identität.

Äh, schön wäre, wenn ich jetzt noch schaffen würde, also so, so ein Ziel, so mindestens zwanzig Kilo wären noch cool. Aber ich weiß nicht, ob ich dann noch wäre, wie ich wäre. (P20:265)

P3 warnt sogar davor, dass eine starke Gewichtsreduktion durch extremes Verzichten 
dem Selbstbewusstsein weiteren Schaden zufügen kann, und bezweifelt, dass Menschen danach akzeptiert und glücklicher sind.

Die Würfel sind gefallen, ich bin jetzt so dick, ist jetzt auch ganz fürchterlich, wenn ich jetzt abnehme, ne, was machen wir denn dann, ne, wenn dann meine viel zu vielen Hautmassen an mir runterhängen und die Krankenkasse sagt, ich bezahl das nicht, die Operation. Was machen wir denn dann, lauf ich dann so rum? Nee, bleib ich auch lieber fett. Macht sich auch kein Mensch Gedanken drüber, ne, wie viel Menschen da, sich dann dazu bereit erklären und da abnehmen [...] gerade bei Frauen, dann sind sie todunglücklich, haben sie nämlich keinen, will sie nämlich kein Mensch, will sie mehr angucken geschweige denn, ja, ne, 'n Mann will sie sich angucken höchstens, wenn's Licht aus ist. (P3:157)

Andere Interviewpartner sind dagegen überzeugt, dass ein konsequenter Umgang mit Diabetes Typ 2 nur in Verzicht und radikaler Diät bestehen kann.

Es hilft also nur, wenn man also eine, sag ich mal äh, gnadenlose Radikalkur macht [...] und da hab ich dann also innerhalb von neun Monaten vierzig Kilo abgespeckt. (P16:0983-0987)

Bezüglich der Körpermaße können auch Neubewertungen (Neu-Normierungen) erfolgen, z.B. bei P32, der sein altes Übergewicht, mit dem er sich früher wohl gefühlt hat, zum Zeitpunkt des Interviews als „krank“ beschreibt.

Bei dem Programm, wo ich da Gewicht reduziert habe, Fotos gemacht: vorher - nachher. Die haben wir noch nicht zu Gesicht ge-, aber ich habe alte Bilder von mir vor diesem Programm gesehen. [...] Ich habe mich selber erschrocken. Weil ich, wenn ich, ich sage mal so, zwei, drei Wochen, fast einen Monat einen Freund nicht gesehen habe, der mich dann sieht: "Was ist denn mit Dir passiert? Bist Du krank?" Dann sage ich: "Das hättest Du mir davor sagen müssen. Da war ich, krank, da war ich richtig krank. Jetzt bin ich gesund." Also viele beziehen das auch, die Gewichtsreduktion, als ob ich krank wäre, dass ich eine Krankheit habe, die mich-. "Nein", sage ich, "ich zahle dafür richtig Geld, dass ich das los werde." (P32:258)

Obwohl diese Aussage wie eine Bekehrung zum ,richtigen“ Verhalten klingt, weist P32 sein früheres „Wohlgefühl“ auch heute nicht als falsch zurück, sondern es hat auch in der Neubewertung Platz:

Früher habe ich nie so gefroren wie jetzt. Das ist ein Nebeneffekt. Aber ein guter Nebeneffekt. Nein, früher, das war, habe ich nicht-. Jetzt sind die Hände kalt, sind die Füße kalt. Ja, Fettreserven sind alle weg. (P32:615)

\subsubsection{Sich Anerkennung „unsichtbarer“ Bemühungen wünschen}

Viele Erzählungen über Begegnungen mit Ärztinnen und Ärzten handeln von enttäuschten Erwartungen und persönlichen Kränkungen, besonders, wenn es um den zumindest subjektiv erlebten Vorwurf geht, nicht genügend zu verzichten. Einige Interviewte wünschen sich, dass bei der Bewertung ihres Verhaltens ihre Altagsanforderungen berücksichtigt werden. P30 scheint nicht erst seit der Diagnose Diabetes mit dem Arzt über das Abnehmen und hohe Blutzuckerwerte zu verhandeln. Die Diagnose 
als eine Art Beweis dafür, dass sie ein scheinbar „krankmachendes“ Übergewicht hat, setzt sie so unter Druck, dass das Verzichten und Abnehmen noch schwieriger wird:

Da habe ich dann eine Zeitlang wirklich immer sehr gut drauf aufgepasst. Und vor allen Dingen, dass das Gewicht auch relativ niedrig bleibt. Aber man vergisst das dann irgendwann wieder, wenn man nicht so betroffen ist. Dass man das eigentlich wegschiebt und eigentlich denkt-. Man hat das zwar im Hinterstübchen: Diabetes ist in der Familie drin. Es könnte dich auch treffen. Aber du bist ja eigentlich noch nicht so, so weit. Und also ich habe es lange Zeit verdrängt. Dann ist ja ' 97 , ist meine, meine Mutter gestorben. Da ist mein Sohn auf die Welt gekommen. Meine Mutter ist gestorben. Und ich sage mal, da bin ich ein bisschen, so in ein kleines Loch reingefallen. Aber man rafft sich dann wieder auf. Und es geht weiter. Und dann sagt auf einmal der Arzt: "Nein, jetzt sind die Werte doch immer konstant schlechter geworden, diese Belastungstests. Jetzt, ähm, stemple ich dich ab als Diabetikerin." 2004. Und das war dann, "Hmm", habe ich erst mal gedacht, "toll". Jetzt, jetzt, jetzt bin ich am Haken, sozusagen. Jetzt geht es noch schlechter. Jetzt muss ich noch mehr drauf achten, was ich esse, wie ich esse, wann ich esse. (P30:137)

In dieser Passage ist P30 offensichtlich zunächst sehr wichtig, ihre Handlungsfähigkeit zu demonstrieren: sie kann auf einen gesunden Lebensstil achten und ist motiviert. Außerdem hatte sie selbst die Kraft, sich aus einer Art depressiver Episode nach dem Tod der Mutter heraus „aufzuraffen“. Gleichzeitig forciert sie aber die „Hypothese“, dass sie keinen Einfluss auf den Verlauf der Krankheit hat, durch ihre restlichen Aussagen: „Man“ - also viele andere Leute auch - „vergisst es ja“. Sie normalisiert ihr Verhalten, die familiäre Disposition zu verdrängen. Die Aussage „Es könnte dich auch treffen. Aber du bist ja eigentlich noch nicht so weit“, lädt Zuhörer dazu ein, sich in P30 hineinzuversetzen, jeder hätte sich ja schließlich selbst einen Aufschub vor der scheinbar unvermeidlichen Erkrankung gewünscht.

Ohne ihre durch den Tod der Mutter noch erschwerten Bemühungen zu berücksichtigen, hat der Arzt scheinbar plötzlich und willkürlich das Urteil gefällt, dass sie Diabetes hat. Dadurch ist es aus ihrer Sicht noch schwerer für sie geworden. Durch das fast schon grausam erscheinende, den Lebenskontext ignorierende Label „Diabetes“ fühlt sie sich der Medizin schutzlos ausgeliefert und wehrlos unter Druck gesetzt, zu verzichten.

Das Gefühl, dass Ärzte die schwierige Aufgabe des Verzichtens nicht im Zusammenhang mit lebensweltlich bedeutsamen Ereignissen und Sorgen sehen, äußert auch P1. Sie wehrt sich gegen den Vorwurf, zu viel gefeiert und dabei nicht verzichtet zu haben, und erklärt die hohen Blutzuckerwerte mit der Angst um ihren Sohn. Sie ist enttäuscht darüber, dass der Arzt scheinbar eine solche Erklärung - letztlich ihr Krankheitskonzept - nicht ernst nimmt. 
Tja, wenn ich mal 'n schlechten Wert hatte [...] da hieß es schon: „Na, was haben sie wieder für Feiern gehabt?" $\mathrm{Ne}$, und das brauch ich gar nicht. Ich sag: „Wir hatten keine Feier, ich brauch mich nur ' $\mathrm{n}$ bisschen aufregen, sofort geht ja auch der Zucker nach oben. Und dann hab ich ' $n$ Sohn, der ist bei der Luftwaffe bei der Bundeswehr und der muss jedes Jahr ins Ausland und wenn der sechs Wochen weg ist, (3) letztes Jahr war er ' $n$ halbes Jahr weg, da hab ich ' $n$ halbes Jahr lang Magengeschwüre gehabt“ [...] - „Warum machen sich solche Gedanken?" Und, und so geht das dann, ich sag: „Wenn ich das nicht machen würde, wäre ich keine Mutter“. Ich sag, es kann kein Mensch mitreden, der das nicht hat, was ich habe. (3) Und man macht ungerne mach ich den Mund auf, weil ich genau weiß, die nehmen dich ja gar nicht für voll. (2) Aber wen soll ich mich anvertrauen, wenn ich noch nicht mal meinen Arzt vertrauen kann? (P1:088)

Von den sichtbaren Werten ausgehend, wird das Verhalten beurteilt und bei „schlechten“ Werten eine moralische Verfehlung suggeriert. In diesem Fall gelingt es der Interviewten jedoch ein moralisch höherwertiges Prinzip aus ihrer Lebenswelt entgegenzusetzen: das der Mutterliebe.

Mehrere weibliche Interviewte argumentieren mit Anforderungen, die im Familienleben an sie gestellt werden, wenn sie sich vor moralischer Diskreditierung schützen wollen. Fürsorge für andere scheint ein anerkannter Grund zu sein, die eigene Gesundheit zurückzustellen.

Wenn ich das jedes Mal von den Ärzten, vor allen Dingen von verschiedenen Ärzten höre: "Hmm, Sie müssten aber mal abnehmen, und so, ne." So nach dem Motto: "Nehme ab, dann hast Du auch die ganzen anderen Beschwerden nicht." Aber das ist ein Widerspruch in sich. Wenn die das sehen könnten, was, was ich tagtäglich mache, würden die das, glaube ich, eher-. [...] Und jetzt, sage ich Ihnen, bin ich schon so weit, dass ich, äh, abwiege. Meine ich: "Das ist kein Leben mehr. Das ist Stress pur." Aber dieser Stress, den verursachen die Ärzte eben halt, weil die sagen: "Du musst doch irgendwie abnehmen können." Meine ich: "Ich mache schon Radel fahren. Ich mache mein Schwimmen [...]

Dazu noch der Haushalt, die Arbeit, ähm, und das ist immer noch zu wenig. Und dann sage ich mir: "Äh, toll!" Soll ich Triathlon machen, oder Sonstiges? Also ich verstehe es irgendwo nicht. Ich mache was. Ich tu was. Und die sagen, das ist nicht genug. Und das ist manchmal sehr frustrierend. Und dann sage ich mir immer: "Warum können die mir nicht sagen: 'Gut, es ist zwar nichts runter gegangen, es ist vielleicht ein bisschen mehr geworden. Vielleicht könntest Du mal..."' Dass man das so, ähm, sagt, dass man einen nicht so verletzt eben halt, ne. (P30:331-335)

P30 fühlt sich erdrückt von den Anforderungen, mit denen sie sich zusätzlich zu ihrer Arbeitsbelastung im Beruf und im Haushalt konfrontiert sieht, und ist gekränkt, dass ihr vorgeworfen wird, zu wenig zu verzichten. Ähnlichen Rollenerwartungen ist P20 ausgesetzt. Für sie ist die Fürsorge ihren Kindern gegenüber ein Grund, sich nicht auch noch durch überzogene Ansprüche zu überfordern. Sie verspürt Druck, ihren Lebensstil zu ändern, hat aber im Gegensatz zu P30 einen in ihre Lebenswelt passenden Umgang damit gefunden.

Und, ähm, habe dann versucht, es mit einem normalen Walking anzufangen. Das heißt also meine Geschwindigkeit, mein Tempo und [habe] dann angefangen, so mit, jeden zweiten Tag. Das konnte ich aber nicht lange aufrechterhalten aus Zeitgründen. Aber dreimal in der Woche. Und, äh, habe dann so peu à peu versucht, jedes Jahr. Ich betone jedes Jahr etwas abzunehmen. Nicht, ich bin nicht jemand, der sagt: "Ich nehme jetzt ab und jetzt müssen dreißig Kilo fallen." Weil ich genau weiß, der Jo-Jo-Effekt, irgendwie holt mich das ein. 
Ich habe ja eben schon gesagt, dass ich also Kinder habe, eine Familie. Meine Kinder noch, zwar schon erwachsen sind und studieren, aber noch daheim sind. Das heißt, so dieses Normale, was so eine Mutter immer tut, wie Essen kochen et cetera, einkaufen und solche Dinge, die habe ich auch heute noch. Und da muss man das immer irgendwie alles so unter einen Hut kriegen: Haushalt, Arbeiten. Man muss überall, in Anführungsstrichen, seine Frau stehen. Habe bis zum heutigen Tag - jetzt mache ich mal ganz schnell einen Sprung - so knapp dreißig Kilo abgebaut. Und bin darauf auch unglaublich stolz. (P20:041)

Auch P20 wünscht sich, dass Ärztinnen und Ärzte ihre Lebenswelt in die Bewertung ihrer Verzichtsbemühungen einbeziehen. Sie vergleicht die Arzt-Patient-Beziehung mit der zwischen Eltern und Kindern und wünscht sich ein entsprechendes Verhalten ärztlicherseits.

Bei dieser Krankheit braucht man einen Arzt, dem man vertraut, der uns so nimmt, wie wir sind, und auch mal über unsere Schwächen hinweg sieht, und uns für unsere Stärken mal lobt. Das ist also einfach so. Wenn man das nicht hat und nicht bekommt, das ist genau wie mit den Kindern: wenn ich immer nur auf den Kopf haue, da passiert gar nichts. Wenn ich aber mal ein leichtes verbales Schulterklopfen vollziehe, dann wirkt das Wunder. Und wir Menschen, egal wie alt wir sind, wir brauchen das. (P20:069-074)

An einer anderen Stelle im Interview schildert sie positive Erfahrung mit ihrer Ärztin, die scheinbar genau ihre Erwartungen erfüllt hat: diese scheint ihre alltagsweltlichen Probleme, z.B. eine an Weihnachten stärkere Versuchung, ungesunde Lebensmittel oder große Essensmengen zu konsumieren, zu kennen und zu verstehen.

Ich war Ende November [bei der Blutuntersuchung]. Und da war mein Blutzuckerwert okay. Dann habe ich so gesagt: "Ich habe jetzt schon ein bisschen Bammel vor Weihnachten und so. Da kann ich mich nicht so richtig zurücknehmen." Und dann hat meine Ärztin gelächelt und hat gesagt: "Nein, ab und zu muss man auch mal über die Stränge schlagen und dann kann man nachher wieder konsequent an sich arbeiten." (P20:074)

\subsection{Subkategorie 3: Verzicht wird geübt}

Während der Erfolg von Verzicht nach festen Kriterien gemessen wird, sind die Regeln der Umsetzung weniger klar definiert. Viele Interviewte fühlen sich damit allein gelassen (vgl. Kap. 5.4.4). Mehrere äußern das Bedürfnis nach ärztlicher Kontrolle ihres Verhaltens. Dies ist temporär durch Diätprogramme im Krankenhaus möglich. Im Alltag dagegen wirkt ärztliche Kontrolle auf viele Interviewte deplatziert und nicht authentisch. Familienmitglieder können kompromisshaft einen Teil der Kontrolle übernehmen und den Verzicht erleichtern. Notwendigerweise stellen die Interviewten aber selbst „Regeln" auf, an denen sie sich bei alltäglichen Handlungen wie Einkauf und Zubereitung von Lebensmitteln sowie Planung von körperlicher Aktivität orientieren. 
Ein deutlich hervortretendes Phänomen ist die Unterscheidung zwischen "guten" und „schlechten“ Lebensmitteln. Dadurch, dass jede Person in gewisser Weise selbst „richtig" und "falsch" bestimmen muss, entsteht ein gewisser Spielraum, in dem sie emotional wichtige Lebensmittel als gesund definieren können.

Laborwerte und Gewicht können als Orientierung dienen, wenn sie durch reproduzierbares Verhalten als beeinflussbar erlebt werden. Ein Beispiel wäre: Jeden Abend Salat essen führt zum gewünschten Blutzuckerspiegel. Dann können Regeln abgeleitet werden, die verlässlich zum Erfolg führen. Erfolgserlebnisse durch Erreichen der gewünschten Parameter können Verzichten erträglich oder sogar zu einer positiven Erfahrung werden lassen. Den Interviewten, die Laborwerte und Gewicht nicht als beeinflussbar erleben, gelingt es nicht, anhand dieser Kriterien Regeln zu entwickeln.

Neben den „sichtbaren“ normativen Erfolgskriterien stehen Lebensqualität oder Wohlbefinden als Ziele im Vordergrund. Wichtig ist auch, dass die Regeln erfüllbar sind. Schon das Einhalten von Regeln ist für viele ein Erfolgserlebnis und stärkt das Selbstbewusstsein.

Oft müssen Interviewte sich von verführerischen Genüssen abschirmen. Dies kann damit einhergehen, dass sie in Konflikte mit gesellschaftlichen Erwartungen geraten und sich ausgeschlossen fühlen (vgl. Kap. 5.4.2). Einige Interviewte entdecken durch den Verzicht aber auch neue Gewohnheiten, die ihr Wohlbefinden erhöhen. Häufig handelt es sich dabei um Rückzugsräume von Familie und Arbeit. Verzicht führt dann nicht zu einem Gefühl des sozialen Ausschlusses und der Einschränkung von eigenen Bedürfnissen, sondern wird zu einer positiven Erfahrung: einer Befreiung.

\subsubsection{Kontrolle von außen: Unterstützung oder Übergriff?}

Alle Interviewten äußern früher oder später Verständnis für die ärztliche Aufforderung, dass sie auf ungesundes Essen verzichten sollen. Einige fühlen sich aber nicht in der Lage zu verzichten, es sei denn, sie würden „gewaltsam“ von medizinischem Personal zu gesunder Ernährung gezwungen.

Herr K., z.B., ((lacht)) hat der mich immer ausgeschimpft. „Liebe Frau“, hat er immer gesagt, „nun hören Sie doch mal auf mich, wenn Sie krank sind, dann kommen Sie [zu] mir, und ich soll Sie gesund machen. Aber Sie hören nicht auf mich, warum soll ich Sie denn noch gesund machen?" Hat er mir immer erzählt, der hat immer geredet. Und letztens, sagt er gar nichts mehr. [...] Ich sage: „Sie schimpfen mich ja gar nicht mehr aus.“ „Ich habe es schon aufgegeben“, sagt er, „Sie hören ja sowieso nicht auf mich.“ ((lacht)) Ja, so sind dann so die Sprüche, ne? Ich weiß das ja selber. Ich sage, „Wo Sie Recht haben, haben sie ja auch Recht.“ [...] Aber eh, dann müssten sie mich, ich 
weiß auch nicht, den Magen verkleinern. Oder irgendwas und so, eh, etwas weg schicken, [...] auf eine einsame Insel. (P27:102)

Das Zugeständnis, dass Ärzte in ihrer Forderung „Recht haben“, reicht offensichtlich nicht aus, um das Verzichten umsetzbar zu machen. Viele Interviewte beklagen, dass sie nicht durch äußere Grenzen zum Verzicht gezwungen werden und der innere Kampf dadurch besonders schwierig ist. Bei einem Beinbruch müssen Menschen auf Mobilität verzichten, wobei die Bewegung entweder gar nicht möglich ist oder sie bei einem Nichtbefolgen unmittelbar und schmerzhaft an die Anforderung erinnert werden. Der Unterschied zwischen einer solchen (akuten) spürbare Grenzen auferlegenden Krankheit und Diabetes Typ 2 ist in Kap. 5.3.3 besprochen worden. Verzicht bei Diabetes Typ 2 ist eine Anforderung, die von außen, manchmal auch von innen gesetzt wird, aber immer eine innere Beteiligung verlangt. Das von P27 gebrauchte Motiv einer Insel, auf der sie vor den verführerischen Genüssen sicher ist und keine eigenen Entscheidungen treffen muss, die anschließend beurteilt werden, findet sich bei P32 wieder, der sich wünscht, sein Arzt hätte inn „eingesperrt“.

Und er hat es auch direkt gesagt, was alles passieren wird. Ist es auch. Wie gesagt, da war es, da lag es an mir. Also, der hätte mich ja nicht einsperren können und sagen: "Ja, jetzt machen Sie es." Eigentlich wäre es schön gewesen, wenn er es gemacht hätte. Da wäre mir vieles erspart geblieben. (P32:679)

Ein paar der Interviewten konnten in einer milderen Form temporär die Kontrolle abgeben und nahmen dann erfolgreich ab. Möglicherweise ist es P16 leichter gefallen, sein Gewicht zu reduzieren, da er bereits im Krankenhaus gezwungen war, eine strenge Diät einzuhalten.

Und ähm, da hab ich dann also mit angefangen, schon im Krankenhaus wurde ja im Krankenhaus also auch, sag ich mal, auf Diät gesetzt, das heißt auf Diät, also auf, sechs Mahlzeiten am Tag, fünfzehn Broteinheiten, wurde gesagt. (P16:0199)

Dieses „auf Diät setzen“ wird während eines Krankenhausaufenthaltes akzeptiert und sogar begrüßt. P16 empfindet den temporären äußeren Zwang als große Hilfestellung. Ebenso scheint es P8 zu gehen, der in einer Kurklinik die Möglichkeit nutzte, sich eine Diät von nur 800 kcal pro Tag verordnen zu lassen.

In der Kurklinik, da gab es die Möglichkeit, normal zu essen, dann gab es Leute, die kriegten Diät und dann gab es welche, die kriegten zwölfhundert Kalorien, tausend Kalorien und achthundert Kalorien, [...] als ich dahingekommen bin, haben die gesagt: „Also, Sie müssen 'n bisschen abnehmen, Sie kriegen zwölfhundert Kalorien“, und da hab ich gefragt, „was gibt's denn noch, wie tief geht's runter?“ - „Achthundert Kalorien.“ Hab ich gesagt: „Dann möcht ich achthundert Kalorien." (P8:0600-0606) 
Auch wenn so ein „realer“ äußerer Druck sinnvoll erscheint, kann er unter gewissen Umständen als unangemessen erlebt werden. Einige Interviewte beschreiben Situationen, in denen medizinisches Personal überraschend in ihrer Lebenswelt auftaucht.

Es war Wochenmarkt und ich war im Geschäft gewesen und meine Frau holt mich da ab und sagt, „Lass uns auf den Markt gehen und 'ne Bratwurst essen, es gibt heut kein Mittag." [... So, wir gehen dann auf 'n Markt und essen diese Bratwurst und die ist in der unmittelbaren Nähe von der Praxis da, vom Doc und da kommt der da raus, geht an mir vorbei, normal hätte er ja auch dezent weggucken können [...] und eine Woche später war ich bei ihm und: „Ich hab schon gesehen, dass Sie eine Bratwurst gegessen haben." Also das ist das, ((lacht)) das ist das Einzige, ne, was mich da so irgendwie-. Ich hab da an dem Stand nie wieder eine Bratwurst gegessen, also so ' $n$ Blödsinn, ne. Warum er das gesagt hat, versteh ich nicht. Ein halbes Jahr später oder 2 Jahre später hat er dann ja sowieso gesagt: „Na ja, musst dir das nicht unbedingt verkneifen.“ (P3:177)

P3 empfindet es offenkundig als Eingriff in seine Privatsphäre, dass der Arzt nicht „dezent wegguckt“, sondern inn sogar eine Woche später noch darauf anspricht. Rechtfertigend führt er im Interview die Umstände der Abweichung von einer „gesunden“ Diät aus: Seine Frau habe inn spontan von der Arbeit abgeholt, die Bratwurst war ein Ersatz für das Mittagessen. Nicht nur steht P3 unter Druck, diesen Kontext im Interview aufzuklären; er fühlt sich aufgrund dieses zufälligen Treffens wie verfolgt und meidet den Bratwurststand dauerhaft. An diesem Beispiel wird zum einen deutlich, dass Ernährung als Privatsache verstanden wird. Zum anderen erscheinen die ärztlichen Ansprüche wenig lebensnah. P3 fühlt sich von seinem Arzt vor ein weiteres Rätsel gestellt, wie er sich eigentlich verhalten soll: Das scheinbar unnachgiebige Tadeln wegen einer einzigen Bratwurst passt nicht zur späteren Empfehlung des Arztes, nicht allzu zwanghaft zu verzichten. Der Arzt wirkt durch diese Widersprüche nicht mehr authentisch, er scheint seinen Bewertungsmaßstab immer wieder zu ändern. Zurück bleibt dennoch eine Scham, bei ungesundem Verhalten ertappt worden zu sein. Eine ähnliche Situation erlebt P8.

Interviewerin: Können Sie sich, mmh, in ne-an 'ne Situation mit Ihrem Arzt erinnern, wo Sie darüber gesprochen haben, dass Sie zum Beispiel abnehmen müssen oder mehr Sport machen?

P8: Vor allen Dingen, ich darf nicht so spät ähm, er hat mich mal, w-w-wir kaufen ((lacht)) gemeinsam, bei einem Getränkehändler ein, und (2) er war in der glücklichen Situation, er kam grade um die Ecke rum mit 'ner Kiste Wasser und ich hatte zufällig 'ne Kiste Bier in der Hand((lacht)) und, da hat er - ohne, d-dass wir uns darüber verständigen mussten - mir deutlich zu erklären gegeben, es wäre hier wohl besser, wenn ich auch ((lacht)) Wasser genommen hätte, dabei hab“ ich inm dann beim nächsten Besuch in der Praxis gesagt: „Das Bier war gar nicht für mich. Sie wissen doch, dass ich Weintrinker bin. Hab ich für meine Jungs ((lacht)) gekauft." ((räuspert sich)) Ja, also abnehmen (2) das ist 'ne schwierige Sache, abnehmen. Zunehmen ist leichter. (P8:0977-0998)

In diesem Fall akzeptiert P8 die Kommentierung seiner Entscheidungen auch im Privaten. Möglicherweise, weil er sich durch die Erklärung, das Bier für seinen Sohn gekauft zu haben, vor einer moralischen Abwertung schützen kann. Interessant ist nicht 
nur, dass überhaupt eine solche banale Situation als moralischer Druck empfunden wird; P8 hat den möglichen Kommentar seines Arztes so weit antizipiert, dass er ihn ohne Worte zu verstehen meint. Vorstellbar ist ja schließlich, dass dieser gar nicht daran denkt, einen Patienten im privaten Alltag zurechtzuweisen, P8 sich aber schon durch die Präsenz des Arztes in Erklärungsnot sieht. P1 „entlarvt“ dagegen ihrerseits den Arzt. Diesem wirft sie Unglaubwürdigkeit vor, nachdem sie seine Frau beim Einkauf „verbotener“ Lebensmittel beobachtet hat.

Ja, und zwar ist das [so]: seine Frau holt nichts, ne, da gibt es wirklich nur gesunde Sachen, und, und, und. Deswegen sind sie auch alle so schlank. Ich sage, hab ich ihm auf 'n Kopf zugesagt: „Das stimmt nicht. Gestern war Ihre Frau auch am [...] Hähnchenwagen und hat Hähnchen geholt.“ ((lacht)) Ich sag: „Das können Sie gar nicht abstreiten, ich kann es sehen, von meinem Küchenfenster aus." Es stimmte ja auch. Also. (3) Und ich sag immer, er kann gar nicht mithalten. Er kann, er weiß doch gar nicht wie's in mir aussieht. (2) Ich hab ihm 'n paar Mal schon auf 'n Kopp zugesagt, ich sage: „Herr Doktor, Sie haben die Krankheiten noch gar nicht gehabt. Sie wissen doch gar nicht, wie 's ist." (P1:525)

In dieser Passage wird deutlich, welche Vorbildfunktion von medizinischem Personal erwartet wird. Umgekehrt wird jeder Fehltritt genüsslich benutzt, um eigenes Verhalten zu rechtfertigen. Ärztinnen und Ärzte sollen die Forderungen, die sie stellen, wenigstens selbst erfüllen können. Sie sollen sich nach Meinung vieler Interviewter aus den konkreten Ernährungsgewohnheiten heraushalten. Familienmitglieder können dagegen den Vorsatz zu verzichten erleichtern, indem sie z.B. in entscheidenden Situationen Kontrolle ausüben bzw. an die eigenen Regeln erinnern.

Also mit den Kindern, die sind mit dieser Krankheit aufgewachsen, auch mit mir, also, die sind da ran-. Das ist für sie normal. [...] Wir stellen ja [bei dem Diätprogramm] um, von dieser Flüssignahrung auf feste Nahrung. Dann darf man ja wieder bestimmte Sachen essen. [...] Am Wochenende saß ich wieder am Frühstückstisch. "Was machst Du denn? Was willst Du denn?" Sage ich: "Ich darf wieder essen." "Nein, nein, nein, nein, nein, nein, Du darfst doch nichts essen." Also, die Kontrolle war fest in ihrer Hand. Dann habe ich ihnen das, äh, was wir gekriegt haben, Punktesystem habe ich dann, äh, gezeigt: Nahrungsmittel, was für Punkte. Was ich essen darf. Hat sie [die Tochter] sich alles genommen, hat sie gleich notiert, alles aufgeschrieben. "Ah, für Vater dürfen wir Salat machen, so- und so viel, drei Tomaten." [...]. Sage ich: "Gut, dann macht ihr heute für mich den Salat, also den ersten Salat." [...] Da habe ich gesehen: Aha, sie verinnerlichen das. Und das jetzt auch mit diesem Punktesystem, das verinnerlichen sie und kontrollieren. Das ist auch gut so. (P32:525)

Ich muss natürlich dazu sagen, dass ich eine Familie habe, die mich eigentlich, also meine Kinder beide, da doch immer unterstützen und sagen: "Wie? Du gehst heute nicht zum Sport? Du musst zum Sport gehen." (P20:091)

Dabei wird Kontrolle eher als Fürsorge, denn als Überwachung begriffen. Während medizinisches Personal den Verzicht im Alltag nur in Form von Ratschlägen und Mahnungen bewerben kann, können Familienmitglieder unmittelbar bei der alltäglichen Ernährung intervenieren. In vielen Interviews wird eine traditionelle Rollenverteilung geschildert, in der die Frau für die Verpflegung der Familie zuständig ist. Ihr wird die 
Verantwortung übertragen, das Verzichten vorzubereiten, der Mann wird zum Konsumenten der schon angepassten Ernährung, er muss nicht selbst „gesunde“ Lebensmittel auswählen.

Also, erst mal das Wichtigste war ja in der ersten Schulung 'ne gewisse Ernährungsumstellung. Das habe ich dann auch gemacht beziehungsweise meine Frau. Da ich nicht kochen kann, bin ich drauf angewiesen, dass meine Frau kocht, aber die hat das hervorragend [...] in [den] Griff gekriegt, äh, da jetzt mehr mit äh, Gemüsen und Säften und, und, Obst und so weiter umzugehen als mit Fleisch und Wurst (3). (P16:0036-0042)

Diese traditionelle Rollenverteilung kann im umgekehrten Fall - wenn die Frau die Erkrankte ist - zur doppelten Belastung werden. Dies hat das Beispiel mit P30 gezeigt, deren Pflichtgefühl, für die Familie schmack- und nahrhaft zu kochen, mit der eigenen Ernährungsumstellung kollidiert (vgl. Kap. 5.3.2). In diesem Zusammenhang geht es auch darum, ob die Familie ihre Ernährungsgewohnheiten anpasst - also wie das betroffene Familienmitglied verzichtet, oder ob mit entsprechendem Aufwand z.B. zwei verschiedene Gerichte für das gemeinsame Essen zubereitet werden. Die Familie erlebt in beiden Fällen eine Einschränkung oder einen Mehraufwand.

Also auch nicht jedermanns Sache, mhm, ähm, dreimal in der Woche Kartoffeln und Gemüse oder Kartoffeln und Quark zu essen, ähm, meine Frau hat das dann also sehr oft mitgegessen, äh, hat aber auch sehr oft, für, äh, die Familie ((schaut zur Seite)) selber was gekocht, was Zweites, ne, mhm, denn habe ich eben halt mein auf mich zugeschnittenes Essen bekommen, und öh für unsern Sohn und für sich selber hat sie dann eben halt, äh, auch mal was anderes gekocht und das nicht nur einmal, sondern auch öfter, weil, ähm, ((schluckt)) das so, wie ich es also esse, äh, sag ich mal, das ist nicht jedermanns Sache, öh, pfff, und viele mögen das also auch nicht so, aber, ((Schulterzucken)) mmh, der Partner muss mitziehen. (P16:1761-1772)

Unabhängig von diesen Schwierigkeiten, die Verzicht im Familienleben betreffen, können Familienmitglieder nie die vollständige Verantwortung für den Lebensstil der Interviewten übernehmen. In den meisten alltäglichen Momenten müssen sie selbst Entscheidungen treffen, ob und wie sie verzichten.

\subsubsection{Laborwerte und Gewicht (nicht) kontrollieren können}

Die ärztlichen Blutzuckermessungen, bei denen der Verzicht bewertet wird, sind von „privaten“ zu unterscheiden, die die Interviewten selbst zuhause durchführen: Bei der ärztlichen Messung werden sie kontrolliert. Beim privaten Messen des Blutzuckerspiegels ist der Druck nicht so stark; es droht kein „Todesurteil“ bei erhöhtem Blutzuckerspiegel. Es dient vielmehr dazu, dass Interviewte ihre Blutzuckerspiegel kontrollieren. Das Messen der Blutzuckerwerte kann dabei helfen, die diffuse Forderung nach Verzicht durch Ausprobieren verschiedener Verhaltensweisen und anschließendem Messen des Effekts („trial and error“) konkret zu realisieren. Viele Interviewte erzählen, 
dass sie sowohl Gewichts- als auch Blutzuckerspiegelmessungen nutzen, um Stück für Stück für sich persönlich herauszufinden, auf welche Dinge sie verzichten sollten und wie viel Verzicht nötig ist.

Also, sehr wichtig ist eben, dass man versucht, sehr viel Fett wegzulassen. Also das macht sich doch stark bemerkbar. Und dann bringt das mit dem Zuckerspiegel reichlich in die Höhe. Also eher könnte man da was Süßes essen, als dass man Fett zu sich nimmt, mindestens habe ich das bei mir festgestellt. (P22:70)

Nicht nur geht es darum, Lebensmittel zu identifizieren, auf die streng verzichtet werden muss, oder die Nahrungsmenge zu begrenzen. Es ist gewissermaßen kontrolliert möglich, Ausnahmen beim Verzichten zu machen, wenn die Parameter dabei im gewünschten Bereich bleiben. Daneben können Ausnahmen durch späteres Ess- und Bewegungsverhalten kompensiert werden, bis erwünschte Werte erreicht werden. P16 kontrolliert auf diese Weise seine Ernährung - bei ihm ist das Gewicht der entscheidende Kontrollparameter, während die Blutzuckerwerte sich kaum verändern.

Ich hab also das ganze Wochenende, äh, immer was gegessen, was ich also sonst nicht esse, ich habe also am Montag vier Pfund mehr drauf gehabt, ne, sofort, ne, und ich bin dann also Montag wieder zu meiner alten Lebensweise äh zurückgekehrt. Habe eben halt, äh, Kartoffeln und Gemüse gegessen, ähm und gestern hab ich Kartoffeln und ' $n$ paar Eier gegessen und das war's dann. Wie ich mich heute Morgen gewogen habe, da war ich also wieder fast auf meinem alten Gewicht runter, [...] man muss [...] gucken, dass man das Gewicht also hält, ne, und ich habe also festgestellt, dem Zucker macht das, äh, der Diabetes macht das eigentlich von Werten her, ((Schulterzucken)) pfff, so gut wie nichts aus, wenn man also auch mal sündigt, ne. (P16:1281-1292)

Für einige Interviewte, die den Eindruck haben, ihre Laborwerte beeinflussen zu können, werden „schöne Werte“ zu einem „Erfolgserlebnis“ (P29:063), zu einem abstrakten Ziel, das zu einem fast schon sportlichen Ehrgeiz führen kann:

Habe also im Krankenhaus schon angefangen mit Abnehmen, und das hab ich also dann weiterverfolgt, und zwar hab ich dann gesagt: ich mache das jetzt, also ich zieh das also durch, ich nehme also ab, bis ich wieder also irgendwo auf vernünftigen Werte bin. (P16:0201-0203)

Ich hab sogar schon mal 'n vier neun geschafft, aber, äh, das ist dann natürlich, das war während der Zeit, wo ich also abgenommen habe, wo ich also, sag ich mal, so gut wie gar nix zu mir genommen habe. (P16:1474-1483)

Ich bin sehr ehrgeizig und, und will natürlich Spitzenwerte haben, und immer, der Doktor sieht das anders, es müssen nicht immer Spitzenwerte sein, wenn es normal ist, ist es auch so, aber ich habe mich da so ' $n$ bisschen vernachlässigt gefühlt. (P29:095)

Für P29, die im letzten der drei Ausschnitte zitiert wird, ist das Erreichen guter Laborwerte wichtiger als für ihren Arzt. Hier entsteht eine interessante Umkehr von dem sonst in den Daten gefundenen Muster: Fühlen sich viele Interviewte von Ärztinnen 
und Ärzten dazu gedrängt, gute Laborwerte zu erreichen, sind sie hier für die Interviewte von größerer Bedeutung als für den Arzt. P29 fühlt sich in ihren Anstrengungen, die sogar „sichtbar“ sind, nicht genügend wertgeschätzt.

Entscheidend für das Verzichten, für das „Eisern bleiben“, ist für P21 die Bestärkung durch die regelmäßigen ärztlichen Kontrollen und die guten Blutzuckerwerte. Der Verzicht ist ihr nur möglich, weil ein positiver Effekt messbar ist:

Also mir wär's schon schwer gefallen, wenn jetzt die Werte erhöht wären trotz meines, also Weglassens, eh weniger leckerer Sachen, sag ich mal, aber so waren die Werte zufrieden, auch dieser Diabetesarzt sag ich mal, bei der Schulung, war mit mir eben auch zufrieden. Die Werte waren okay und so mach ich also im Grunde weiter. (P21:277)

P21 (626) fasst ihre Vorstellung von Ernährung unter folgendem Satz zusammen: „Aber meine Devise ist, wie gesagt, von allen ja, aber wenig. Und wirklich in Maßen.“ In diesem Prinzip deutet sich die Hoffnung an, dass es gelingen kann, sich trotz einer Diät frei zu fühlen. Eine Voraussetzung dafür ist die von P21 sich selbst zugeschriebene Eigenschaft, „maßvoll“ zu sein. Mit der Erarbeitung der persönlichen Wertschätzung geht in gewisser Weise auch eine „Absolution“ durch die Ärztin einher, die die Auflage zu verzichten lockert (vgl. Kap. 5.6.1). Fast schon schildert P21 es als Triumph, wenn sie es sich erlaubt, von ihrem Ernährungsplan abzuweichen - letztlich aber mit der Zustimmung durch die Medizin, symbolisiert in den „Werten“:

Jahh und das hat ja bewiesen, bis jetzt, dass, wenn ich denn noch mal rüber gehe nachmittags zum Bäcker und $\mathrm{da}$, der hat dann am Mittwoch frische Windbeutel, ne, denn esse [ich] auch mal ' $\mathrm{n}$ Windbeutel, ja? Und lass mir den schmecken. Ja und die Werte zeigen ja, dass so nicht falsch ist. (P21:534)

Die Laborwerte, die - wie bereits erläutert - von medizinischem Personal als Erfolgskontrolle, aber auch von den Kranken als Verhaltensorientierung herangezogen werden, scheinen oft, aber nicht immer die erwarteten Effekte des Verzichtens abzubilden. Zum Teil erweisen sich die Ergebnisse der Blutuntersuchungen geradezu als unlogisch bezogen auf das Verzicht-Paradigma („Man muss nur verzichten - dann erreicht man den erwünschten Blutzuckerspiegel“). P30 kommt im Laufe des Interviews immer wieder auf diese Enttäuschung zurück:

Ja, ich esse morgens eine halbe Scheibe Schwarzbrot mit ein bisschen was drauf und meinen Kaffee, Milchkaffe. Und habe aber nach zwei Stunden immer noch, also 180 oder 200 Zucker. Und nach zwei Stunden, da sagt man sich, müsste er eigentlich weiter [gesunken sein], für die Menge. Andere essen verbotenerweise ihre Semmel und haben bei weitem nicht so viel wie ich. Und das, obwohl ich schon eben halt die Tabletten nehme. Und das ist mir manchmal unverständlich. [...] Okay, manchmal esse ich ein bisschen zu viel. Wenn ich doch noch eine zweite Portion esse, dann weiß ich, dass er dann hoch ist, ne. Aber bei einigen Sachen, das kommt auf die Tagesform 
auch drauf an. Manchmal ist er dann, esse ich zwei Tage hintereinander [das Gleiche], habe ich zwei unterschiedliche Ergebnisse. Das ist eben halt so. Aber ich bin jetzt schon fast so weit, dass ich mir sage, obwohl ich nur auf eigentlich Diät und Tabletten lebe, dass ich schon fast am Abwiegen immer bin. Dass ich, äh, die Portionsgrößen mir abwiege, um eben halt nicht zu viel zu bekommen. Und das ist gar nicht mal so einfach. (P30:093)

Da sage ich auch immer: "Ich esse weniger, und trotzdem nehme ich zu." [...] Ich habe schon gesagt, jetzt, äh, werde ich nur noch von Luft und Liebe mich ernähren. [...] Jedes Mal, wenn ich auf die Waage mich hinstelle, und dann sehe ich: äh, Scheiße! Schon wieder zugenommen. Also das frustriert schon, weil man so vieles versucht, und so viel macht. Und dann kein Ergebnis, sondern so ein Negativergebnis dann hat. (P30:331)

Nicht einmal das genaue Abwiegen der Lebensmittel - im Sinne einer "Gegenprobe“ zu den gemessenen Laborwerten - führt P30 zum Erfolg. Ironisch bietet sie die Lösung des Problems an: sich allein von „Luft und Liebe“ zu ernähren, da sich aus den Veränderungen ihrer Laborwerte offensichtlich keine vernünftigen Alltagsregeln ableiten lassen.

Besteht dauerhaft der Eindruck, das Gewicht bzw. die Laborwerte nicht durch Verzicht beeinflussen zu können, kann eine Abkehr von "den Werten“ (und damit auch vom ärztlichen Maßstab) die Folge sein.

Also, seitdem ich eigentlich in dieser Selbsthilfegruppe bin, ist zwar mein Zucker immer schlechter geworden, statt besser, mit diesen ganzen Tipps, und hier und da und so, ne. Aber ich sehe eben halt auch, ähm, die Tipps, die gegeben werden, die sind goldrichtig und gut. (P30:302)

P30 hat in der Selbsthilfegruppe konkrete Hilfestellung erhalten, wie sie verzichten kann, erlebt aber, dass sich ihre Bemühungen nicht in den Laborwerten zeigen. Dennoch hält sie an den Tipps fest, von denen sie überzeugt ist, dass sie ihr guttun. Statt sich an den Werten zu orientieren, „hört" sie auf ihr Wohlbefinden.

\subsubsection{Regeln formulieren: „gesunde“ und „ungesunde“ Lebensmittel}

Die Auswahl und Zubereitung von Essen spielt (erwartungsgemäß) eine große Rolle bei Diabetes Typ 2. Häufiges Thema in den Interviews sind Lebensmittel. Diese werden, wann immer die Interviewten sie erwähnen, bewertet. Ähnlich dualistisch wie die Bewertung der Körpermaße mit der Einteilung in "die Dicken“ und "die Dünnen“ stellt sich die Bewertung der Lebensmittel dar. "Gesunde“ stehen „ungesunden“ Lebensmitteln gegenüber. Ungesunde Lebensmittel werden häufig im Zusammenhang mit Verboten genannt (vgl. Kap. 5.4.1, „Mein Sonntagsbraten ist verboten“ [P2:055]; „Und dann hat er empfohlen, also nicht mehr so viel Weißbrot“ [P21:237]). Daneben erwähnen manche Interviewte ungesunde Lebensmittel, wenn sie von vergangenen Gewohnheiten erzählen: 
Dann hab ich also, wenn ich an die Tankstelle fuhr, mir Süßigkeiten [gekauft], so kleine „Schmeckewöhlerchen“ [...] Mittag gegessen hab ich öh, im Vorbeilaufen irgendwo, äh, an 'ner Pommesbude mal 'ne Currywurst oder so was in der Form und nachmittags gab's dann noch 'n Stückchen Kuchen eventuell. Also alles das, was eigentlich gut schmeckt äh, aber was man eigentlich nicht jeden Tag essen sollte und das hab ich also jahrelang gemacht, dazu immer noch gerne [...] 'n schönen Schoppen Bier getrunken ähm, ((zuckt mit den Schultern)) naja, und das ist dann also [...] diese ungesunde Lebensweise gewesen, die das Gewicht hochgetrieben hat, äh und die auch im Endeffekt, äh, wenn ich das also heute so sehe, ähm, die Diabetes verursacht hat. (P16:1041-1053)

Dem Interviewten P16, der, wie er an anderer Stelle berichtet, zum Zeitpunkt des Interviews sehr auf eine gesunde Ernährung achtet, bezeichnet die früher genossenen Speisen heute als ungesund und als Ursache seiner Krankheit. Ein anderer Interviewter bewertet sein früheres Ernährungsverhalten ebenfalls als schlecht.

Ich, wie gesagt, also ich habe [nach der Diagnose erst einmal] zugenommen. Es war wirklich, ich, äh, es waren ein paar Kilos, die dann drauf kamen. Die Bewegung hat gefehlt eindeutig. [...] Arbeiten, äh, Familie, am Wochenende schön Grillen. Also das Essen, nichts verzichtet, was ein Fehler war, sehr fettig, sehr viel Fleisch. Was, äh, seit drei Monaten esse ich kein Fleisch. Es geht, wunderbar. Ich habe immer gedacht: wie können Vegetarier leben? (P32:382)

Beide, P16 und P32, stellen ihr Verhalten in einen Kausalzusammenhang mit der Diabeteserkrankung (P16) bzw. mit der Gewichtzunahme (P32). Beide erzählen aber auch, dass sie ihr Verhalten inzwischen geändert haben.

In den Interviews werden auch viele Lebensmittel als gesund bewertet, z.B. wenn ein Interviewter berichtet, „dreimal in der Woche Kartoffeln und Gemüse oder Kartoffeln und Quark zu essen“ (P16). Er erklärt, dass das „auch nicht jedermanns Sache“ ist und seine Frau dann manchmal noch eine weitere Speise für sich zubereitet. Gesundes wird häufig als etwas charakterisiert, das keinen Genuss bietet und das Menschen ohne Diabetes nicht essen würden. P21 zeigt, dass gesunde Ernährung zu Konflikten führen kann:

Obwohl mein Mann nicht so begeistert ist, aber Gemüse hat wirklich zugenommen und-. Schwer fällt mir wie gesagt noch, eh, so Fleisch wegzulassen, weniger Fleisch [...] Gerichte. ((lachend)) Weil man nun kochen muss für 'n Mann und dann, ist schon (2) hart ((lacht)). (P21:431-435)

Aus dem letzten Zitat lassen sich Rückschlüsse auf die Vorstellung und Bewertung von Lebensmitteln ziehen. Insoweit es P21 problematisch erscheint, für ihren Mann etwas ohne Fleisch zu kochen, deutet sich die Vorstellung an, dass Fleisch notwendigerweise zur Ernährung von Männern gehört bzw. sie, als Versorgerin der Familie die Aufgabe hat, „Nahrhaftes“ zu kochen, und deshalb bezüglich ihrer eigenen Diät vor einem Dilemma steht. Umgekehrt ist aber auch eine aus dem Rollenkonflikt gewon- 
nene (unbewusste) Rechtfertigung möglich: Weil sie ihren Mann nicht auf etwas verzichten lassen will, muss sie sich selbst nicht einschränken. Das folgende Zitat spiegelt noch eine generationstypische Vorstellung von Nahrungsmitteln:

Und da bin ich noch nicht so eisern. ((lacht)) Da ess' ich zu gerne Mittag auch, und, wie gesagt, mein Mann auch. Wie sind denn, gro- starke Kartoffelesser, ja? (P21:546)

Diese scheinbar nebensächliche Bemerkung macht darauf aufmerksam, dass Kartoffeln in der Diät „verboten“ sind, aber trotzdem verzehrt werden, weil sie gut schmecken und „stark machen“ (dieser assoziative Zusammenhang gilt vermutlich auch für Fleisch, s.o.). Von diesem Lebensmittel, das im klassischen Sinne „nahrhaft“ ist, wird der Erfahrung von P21 nach bei Diabetes abgeraten. Dagegen werden mögliche Alternativen durch Formulierungen wie „irgend 'n Knäckebrot zu knabbern“ (vgl. Kap. 5.4.2, S. 53; P21:265-269) in ihrer Substanz und Schmackhaftigkeit desavouiert. Als Zwickmühle empfindet P8 den Verzicht von Zucker und Salz:

Also Zucker darf ich nicht zu mir nehmen, in keiner Weise wegen Diabetes zwei, Salz darf ich nicht wegen der Herzgeschichte, beides sind aber, ääh, wohlschmeckende Nahrungsmittel, die einen sollen sogar glücklich machen, Zucker, Schokolade und so, und Salz, gehört also an verschiedene, ääh, Dinge, wo was also gar so ganz gar keinen Geschmack hätte (2) und das habe ich mittlerweile gelernt, völlig ohne Salz auszukommen,[...] , aber jedenfalls weiß ich mittlerweile dass ein gekochtes Ei mit Maggi viel besser schmeckt als mit Salz. (P8:0512-0518)

Bei P3 finden sich ebenfalls generationstypische Vorstellungen von Lebensmitteln: hier ist die Erfahrung vom Hunger des Vaters in der Kriegszeit ein Grund für die (anerzogene) Gewohnheit, möglichst viel und nahrhaft zu essen.

Von mittags 12 bis nachmittags dreie, viere, ja, ist doch klar, so, ne und alles so Kerlchen wie ich, 4 Jungen, joa, da wurde schon ganz schön was verputzt. So ' $n$ Kartoffelpuffer ist ja nicht so schlimm, huu, ist doch schlimm, hab ich gehört ((lacht)). Ja, es war, die Zeiten waren da anders und es ist einfach so gekocht worden und, dass das heute, heute, äh, ungesund ist, das war damals auch ungesund, das hat niemand eingesehen oder, ja die kamen, was weiß ich, die Väter kamen aus dem Krieg, verhungert ohne Zähne, wegen Vitaminmangel, erinnere ich mich so an meinen Vater. (P3:153)

In diesem Zitat deutet sich an, dass es schwierig für viele der Interviewten ist, wenn sich die von außen gegebenen Regeln und die Bewertung, welche Lebensmittel "gut“ und welche „schlecht“ sind, verändern. Schon allein deshalb ist es unumgänglich, eigene Regeln zu formen und immer wieder an neue Informationen anzupassen. Man könnte diese Regeln auch als praktisches Wissen verstehen, dessen Gültigkeit immer wieder in Frage gestellt wird, weil neue Erkenntnisse dazu kommen. Für P8 muss es eine große Enttäuschung sein, als er die Regel erfüllt hat, mehr Obst zu essen, und eine Obstsorte gefunden hat, die er auch noch mit Genuss isst - dann aber erfährt, 
dass ausgerechnet diese Früchte sich nicht so günstig auf seinen Blutzucker auswirken. Erneut muss er einen Ersatz für ein erst als „gesund“ bewertetes Nahrungsmittel finden. So versucht er sogar, gar kein Obst mehr zu essen und sich mit Cornflakes im Jogurt zu behelfen, wobei die Formulierung „trockene“ Cornflakes erneut an Verzicht erinnert und elegant deren Zucker unterschlägt:

Was ich gerne ääh, gegessen habe ist, also ich esse nur so 'n Becher, äähm, Joghurt, und da rein, so 'ne Handvoll, irgendwelches klein geschnittenes Obst, aber liebend gern. Die Brombeerzeit kommt bald wieder. Brombeeren [...], das weiß ich nun auch wieder durch die Schulung: Gerade bei Brombeeren, die mir am besten schmecken, da ist nun wieder die meiste Süße drin. Mhmm, also, muss ich das wahrscheinlich auch lassen[...]. Im Moment bin ich dabei, gar kein Obst mir da rein zu machen, sondern einfach, ääh, so trockene, ääh, Cornflakes (2). Das sind alles so Versuche, um von dem Zuckerwert runterzukommen. (P8:0627-0636)

Essen kommt eine hohe emotionale Bedeutung zu. Es scheinen gerade die Lebensmittel „verboten“ zu sein, die eine besondere Freude auslösen (vgl. Kap 5.4.1). In dem folgenden Beispiel wird deutlich, dass der Genuss bestimmter Lebensmittel schöne, persönliche Erinnerungen transportiert. Eine bestimmte Süßigkeit wird hier zum Vehikel für das Wiederbeleben positiver Gefühle, die aber nur für einen Moment erlaubt werden, bevor der „Schweinehund“ wieder „verdrängt“ werden muss:

Überall liegt natürlich für die Kinder Schokolade und zu Ostern, Osterhasen und so 'n Zeug rum, ne, da kommt man natürlich auch in die Versuchung, ich habe, jetzt zu Ostern, da hatte meine Frau Osterhasen gekauft, nicht drauf geachtet. Da war einer dabei, ääh, mit dunkler Schokolade, und ich ((lacht)) hatte grade vorher gelesen, dass dunkle Schokolade, wenn überhaupt, für 'n Diabetiker besser sei als andere, und dann hat meine Frau gesagt: „Ja denn, die Kinder mögen das sowieso nicht, denn ist der für dich." Denn hab ich den da, ((räuspert sich)) naja vierzehn Tage ((lacht)) angeguckt, und dann bin ich da richtig mal drangegangen, habe oben die Ohren abgebissen, und dann ist das natürlich ne, ja 'ne schöne, Erinnerung an früher, da hatte ich 's noch ein bisschen öfter gegessen, aber dann muss man den inneren Schweinehund natürlich, ääh, verdrängen und nicht dabei bleiben. Dann muss man das zur Seite legen und später noch mal so 'n Stück irgendwann. (P8:0646-0652)

P8 erzählt, wie er den Schokoladenhasen zwei Wochen lang im Sinn hatte und sich ein Verlangen danach aufgebaut hat, bis er schließlich „richtig drangegangen“ ist und „die Ohren abgebissen“ hat. Diese Erzählung bringt den starken inneren Konflikt zum Ausdruck, mit dem er kämpfen musste. Deutlich wird, wie sehr die banal wirkende Nahrungsaufnahme zu einem unterschwelligen stetigen moralischen Problem wird, wenn es um eine - letztlich ja von P8 selbst - als „ungesund“ definierte Speise geht.

Andere Interviewte erwähnen ungesunde Lebensmittel eher, wenn sie sich vom „Schlechten“ Ernährungsverhalten anderer abgrenzen. Sie wirken bemüht, zu betonen, dass sie sich auch vor der Erkrankung gesund ernährt haben:

Naja, ein Vorteil ist natürlich, dass ich keine Süßigkeiten esse. Und, oh, ich esse auch mal ein Stückchen Schokolade, wenn meine Enkelkinder da sind. Okay. Dann habe ich auch so was zu Hause. Sonst habe ich sowas gar 
nicht zu Hause, weil ich das einfach nicht brauche. Ich esse da lieber einen Apfel für. Und, äh, wie gesagt, ich esse dann schön Gemüse [...]. Sehe auch zu, dass ich nicht zu fett esse. (P15:0424)

Ähm, ich habe nie, auch vorher, sage ich mal, nie fett gekocht. Also sage ich mal, fett wirklich vom Wort Fett. (P21:041)

In diesen Aussagen präsentieren sich die Interviewten so, als falle es innen nicht schwer, auf ungesunde Lebensmittel zu verzichten. Zwei weitere Zitate passen in diese Reihe. Darin deutet sich neben der Abgrenzung von ungesundem Essen noch ein weiteres Muster an. P21 wertet Torte ab und stellt gleichzeitig Kuchen als etwas Gesünderes dar. Bei Schokolade besteht sie auf eine genaue Unterscheidung zwischen verschiedenen Sorten. Für P27 sind Brathähnchen gesund.

$\mathrm{Na}$ ja und Torte nicht zu essen fällt mir sowieso nicht schwer. War nie mein Ding. Aber so schön` Kuchen (P21:534)

Ja und, eh, wie gesagt, mir fällt es nicht schwer. [...] Ich [...] esse so gerne schwarze Schokolade, also und das fiel mir denn-, kam mir zugute, wie gesagt. Manch einer isst ja eher lieber Vollmilch. (P21:534)

Eine Scheibe Brot und dann bin ich bedient. Und mein Mann, gestern hat er halbe Hähnchen mitgebracht [...]. Da haben wir ein halbes Hähnchen, waren wir auch bedient, brauchten nichts dazu, ne? Reicht ja auch. Ne? Das reicht. Wie früher musst du sein. Oh! Da habe ich Suppe gekocht. Dann habe ich Gemüse und Kartoffeln und Fleisch und ah ich war fleißig, ich habe zwei Truhen. Liegt voll Fleisch. [...] Mein Sohn, der grillt wieder. Der ist wieder nur für 's Grillen. Ich grille einmal und dann ist Schluss. [...] Wenn dann mal ein Bratwürstchen, aber dann 'ne Krakauer. [...] Ich sag mal, man hat doch gar nicht viel [...]. (P27:110)

In diesen Beispielen zeigt sich, dass die Bewertung von Lebensmitteln subjektiv ist und Lebensmittel, die von emotionaler Bedeutung sind, als gesund definiert werden können. Subjektive Regeln, was gesund und ungesund ist, bieten eine praktische Orientierung für den Verzicht. Weißbrot wird z.B. durch das "gesunde Vollkornbrot" ersetzt, es wird „immer frisch“ gekocht (P30); genauso aber kann es als gesünder empfunden werden, statt Torte „nur Kuchen“ zu essen (P21) oder wenn „einmal“ gegrillt wird, dann nur „ein Bratwürstchen oder 'ne Krakauer“ (P27).

Einigen Interviewten gelingt es, emotional bedeutsame Dinge als „gesund“, oder zumindest in der Abgrenzung zu anderen als „gesünder“ zu definieren. Ein Beispiel, in dem es einem Interviewten besonders gut gelingt, Regeln zu entwickeln, die zu seinen Bedürfnissen passen, und sich von „maßloseren“ Menschen abzugrenzen, findet sich in folgendem Zitat:

In den ersten, äh, Besprechungen [in der Diabetes-Schulung], wo dann so zehn, fünfzehn Leute zusammensitzen, gibt's natürlich [...] jede Menge Hemmungen, erstmal wird man dezent, aber bestimmt nach dem Gewicht gefragt, und die meisten Diabetiker haben Übergewicht. Ich auch, aber nicht so viel wie die andern hatten, [...] Eine Dame hat sich da schwer getan, ihr Gewicht zu sagen oder auch ((lachend)) ihr Alter zu sagen, das gibt's ja auch [...] 
Naja und dann, werden die Essgewohnheiten, äh auch angesprochen. Wer gibt denn gerne zu, dass er jeden Tag fünf Flaschen Bier trinkt? (2).

Also ich habe zugegeben, dass ich keinen Schnaps und kein Bier trinke, aber liebend gern Wein und da speziell Rotwein, und dazu hat mein Arzt mir sogar geraten, in Maßen, weil das ääh, gut für die Verdünnung des Blutes ist. Gott sei Dank hat mich keiner gefragt, ob ich mich an die beiden Trinkeinheiten, die man so nennt, nämlich zwei [empfohlenen Einheiten] halte, ääh, sonst hätt ich sagen müssen, „Nee das wären noch mindestens mal vier oder fünf." ((lacht)) (P8:323-335)

P8 erzählt, dass viele Menschen in der Diabetes-Schulung sich damit schwertun, offen über ihr Gewicht und ihre Ernährungsgewohnheiten zu sprechen. Zunächst scheint es, als würde er sich frei von diesen Hemmungen fühlen und diese belächeln. Interessanterweise betont er, dass er weniger Übergewicht hatte als die anderen. Letztlich gelingt es inm auch nur „zuzugeben“, dass er Rotwein trinkt, zu dem ihm der Arzt sogar noch „geraten“ hat. Die „Regel“, dass Rotwein erlaubt ist, übernimmt er gern, da er sie gut erfüllen kann und sie seinen Bedürfnissen entspricht. Die Menge möchte er lieber verschweigen. An anderer Stelle erwähnt P8 den Genuss von Rotwein noch einmal. Im folgenden Zitat findet sich ein Argumentationsmuster, das auch viele andere Interviewte für sich reklamierten: zugunsten von Wohlbefinden und Lebensqualität ist auch ungesundes Verhalten erlaubt.

Ich trinke gern mal 'n Glas Rotwein und genieße dazu, ääh, dunkles Brot. Die Franzosen essen ja Weißbrot dazu, aber das, hat mir meine, ääh, die Mitarbeiterin von meinem Hausarzt denn auch ausgeredet, [kein] Weißbrot mehr zu essen, dann esse ich Graubrot dazu und 'n schönen, naja nicht ganz fettfreien Käse, denn da fehlt auch wieder der Geschmack, aber, ein bisschen was muss man ja auch, ähm, sündigen, sonst hätte das Leben ja gar keinen Zweck mehr. (P8:0537-0539)

Viele Interviewte scheinen das Gefühl zu haben, dass ihnen das allgemeine (Grund-) Recht auf Genuss aberkannt wird (vgl. Kap. 5.4.1). Dagegen wenden sie sich zum Teil mit drastischen Worten:

Man sollte nicht gleich denken: "Ich habe jetzt ich-weiß-nicht-wie viel-Jahre das falsch gemacht". Ich muss nicht denken, das muss ich jetzt innerhalb von vier Wochen komplett umgekrempelt haben. Das funktioniert nicht. [...] Und ich kriege auch mal einen Jieper, dass ich mal gesagt habe, dieser Tage: "Kann nicht mal einer irgendwie, ich habe mal Hunger auf Pommes Frites". Und dann haue ich mir da auch mal ein Löffelchen Mayonnaise drauf und lebe mal völlig dekadent. Aber dann habe ich meinen Jieper wieder befriedigt und habe gesagt: "Das habe ich mal wieder gegessen. Jetzt geht es mir gut. Ah, das tat gut." Wenn ich es dann so fast aufgegessen habe, dann habe ich schon mal gedacht: "Das hättest Du Dir ja eigentlich klemmen können." Aber wenn man nicht mal ab und zu diesem nachgibt, dann kasteien wir uns doch. Dann haben wir doch gar nichts mehr vom Leben. Da können wir uns mal gleich erschießen. (P20:284)

In diesem Zitat stellt sich die Auseinandersetzung mit dem Thema Verzicht in großer Komplexität dar. "Natürlich" isst P20 auch die unerlaubten Lebensmittel gern. Damit stellt sie heraus, dass das Verlangen danach letztlich normal ist. Einen "Jieper", gar einen "Hunger" nach etwas zu haben, ist ebenfalls etwas, was sie nicht steuern kann 
und das demnach normal (im Sinne von „menschlich“) zu sein scheint. Fast paradox erscheint der Satz, in dem P20 beschreibt, wie sie "völlig dekadent" ein "Löffelchen Mayonnaise" zu sich nimmt. Darin wird der Druck deutlich, der auf ihr lastet, sich maßvoll zu verhalten bzw. sich als maßvoll darzustellen. Das Wort "dekadent" spiegelt ihren hohen moralischen Anspruch - oder den von außen erwarteten Anspruch. Eine doppelte Wiedergutmachung geschieht dadurch, dass sie die „Dekadenz“ auf ein „Löffelchen“ reduziert, zumindest verbal, und dass sie danach ein Wohlgefühl verspürt und ihr Verlangen gestillt hat, sodass sie erst einmal nichts Verbotenes mehr zu sich nehmen wird. In dem Moment kann sie sogar wieder scheinbar rational darüber nachdenken, dass sie es auch hätte sein lassen können. Davon wendet sie sich allerdings ab. Ein schlechtes Gewissen hat keinen Platz, denn sonst hätte es keinen Sinn mehr zu leben. In diese Aussage bezieht sie die Zuhörenden mit ein, indem sie ein "wir" formuliert. Dadurch wird ihr Bedürfnis wiederum zu etwas „Natürlichem“.

Man könnte diese Episode auch so kommentieren: Einem letztlich trivialen Ereignis, einem bekannten, sicher durchschnittlichen Verhalten kommt hier nur deshalb Beachtung zu, weil der argumentativ betriebene Aufwand als fast schon dramatisch zu bezeichnen ist und einen Blick auf den normativen Druck eröffnet, der auf der Interviewten bereits bei so kleinen Alltagsentscheidungen lastet.

Dauerhaft auf alle Genüsse zu verzichten, passt nicht in die Alltagswelt der meisten Interviewten, z.B. beim Verlangen nach Pommes Frites statt der "langweiligen Kartoffeln" (P30:264). Die Wunschobjekte scheinen häufig verbotene, ungesunde Lebensmittel zu sein.

Pommes ist natürlich was sehr Leckeres, ne. Da kann ich nicht widerstehen. Also, [...] dann sage ich auch manchmal: "Oh, nein, nicht diese, diese langweiligen Kartoffeln. Ich will jetzt mal wieder Pommes haben." Muss manchmal sein. Weil, wenn man die ganze Zeit immer verzichtet, da kriegt man dann so einen Appetit drauf. Aber man, äh, ich mache das dann einmal, und dann langt das aber auch dann wieder. Das, äh, das hat die Frau [aus der Selbsthilfegruppe bzw. aus der Schulung] auch zu mir mal gesagt, oder beziehungsweise zu uns allen: "Sündigen dürfen wir alle mal. In Maßen", sagt sie. "Weil das ist besser, als sich immer nur zu sagen: 'Nein, Du darfst das nicht, Du darfst das nicht."' (P30:264)

P30 erwähnt zunächst, dass Pommes Frites „natürlich etwas Leckeres sind“. Ähnlich wie P20 im vorausgehenden Zitat erklärt P30 es außerdem zu einem „natürlichen“ Vorgang, dass sie umso größeren Appetit auf etwas hat, je mehr sie es sich vorenthält. In diesem Fall wird der innere Konflikt dadurch erleichtert, dass das ungesunde Essen 
auch in der Außenansicht, nämlich von der Expertin aus der Selbsthilfegruppe erlaubt ist.

Gegenüber der schier unendlichen und stereotypen Forderung nach Verzicht, erscheint die Regel, die P32 in einer Kur befolgen lernt, widersprüchlich und angenehm:

So was mir als erstes da [in der Kur] aufgefallen ist, und beim Psychologen war das, der hat ein schönes Schild gehabt, da stand drauf "Verbote sind verboten". Da habe ich inn drauf angesprochen: „Verbote sind verboten, das klingt sehr schön. Was für Verbote?“ - „Alles, was man sich, also, zum Beispiel, bei der Ernährung..."Wo ich gesagt habe: 'Das will ich nicht, das will ich nicht.' „Das ist verboten“, hat er gesagt, „Sie dürfen essen, alles, „alles“ in Anführungsstrichen, in Maßen, abgestimmt mit ihrem Blutzucker." Das war für mich wirklich, äh, ein Wendepunkt, wirklich ein Wendepunkt, weil ich immer verzichtet habe: das will ich, esse ich nicht, das will ich. Und dann kam der Tiefpunkt, wo ich dann gesagt habe: „Ach, ist mir doch egal.“ Und dann ist das alles dann so hinten drum wieder in die alten Strukturen. (P32:234-238)

P32 rechtfertigt einen weniger restriktiven Ernährungsplan damit, dass der Druck, zu verzichten, so groß werden kann, dass man es völlig aufgibt, sich gesund zu ernähren. Auch P3 erfährt von medizinischem Personal, dass eine Orientierung am eigenen Wohlbefinden erlaubt ist. Dabei kommt in diesem Beispiel hinzu, dass die medizinische Autorität Wohlbefinden auch mit einer positiven Beeinflussung der Laborwerte und Blutdruckwerte in Verbindung bringt.

Ob das der Doktor so richtig gesagt hat oder ob das da im Krankenhaus so 'ne, so 'ne Ernährungsberaterin war, äh, vielleicht vermisch ich das auch alles so: mit diesem Verkneifen und, dass das nicht gut wäre, ähm, ja, dass man sich ja eben auch wohl fühlen soll bei der ganzen Sache und wenn man [...] gegen seine Lust arbeitet, das tut dem [...] Blutdruck [...] und dem Zucker, auch nicht unbedingt gut. Also man soll enthaltsam [sein], ja, und man sollte das ein oder andere von vornherein ausschalten, aber man sollte sich nicht wirklich was verkneifen. (P3:179)

P20 stellt diese Assoziation ebenfalls her: ihrer Erfahrung nach hängen subjektives Wohlbefinden und "gute Werte" unmittelbar zusammen.

Ich finde immer, es hat auch was mit der Psyche zu tun: Wie bin ich drauf? Wie geht es mir? Ich liebe unglaublich das Frühjahr. Dann geht es mir unglaublich gut. Und merkwürdigerweise ist dann auch mein Zucker viel besser, weil, ähm, ja, ich weiß es nicht. Irgendwie hat alles was damit zu tun. (P20:061)

An anderer Stelle schildert sie ihren Versuch, diese Erkenntnis direkt in eine Alltagsregel umzusetzen: Sie will ihren Essverhalten unmittelbar an ihre Bedürfnisse anpassen. Sie hat den Eindruck, dass sie mit einer positiven Grundstimmung leichter abnehmen kann als mit Reuegefühl.

Man muss dann auch für sich so ein bisschen so ein Wohlbefinden finden. Ich erfinde immer wieder was Neues. [...] Und habe mir dann überlegt: Du hast jetzt gut gefrühstückt. Ich habe auch, wie gesagt, auf der Arbeit immer eine Möglichkeit, mir was in den Kühlschrank zu stellen oder so, und: versuche es doch mal einfach, nach Bedarf zu essen über den Tag. Sprich, wenn ich merke, ich habe Hunger, dann esse ich auch was. Aber ich esse nicht, weil zwölf Uhr ist. Weil das finde ich völlig Banane. Wenn ich also irgendwann auf der Arbeit merke, um elf Uhr, ich habe Hunger, dann esse ich um elf Uhr was [...]. Mal Knäckebrot mit Quark [...]. Oder, ach, weiß ich nicht, wo ich Lust drauf habe. Ich gehe auch mal, da ist unweit also so ein toller Schlachter. Ich gehe auch mal einfach dahin 
und, äh, kaufe mir mal drei Bratrollmöpse, weil ich da mal jetzt einen Jieper drauf habe. [...] Und ich habe festgestellt, wenn ich was esse, wo ich Appetit drauf habe, was ich nicht bereue, was also, klar, immer in meine Geschichte passt, dann nimmt man wahrscheinlich auch nicht so schnell von zu, wenn man sich nicht so übermäßig da betätigt beim Essen. Und habe aufgrund dieser Tatsache, denke ich mal, einfach mal ein bisschen Gewicht verloren, ohne dass ich das gemerkt habe (P20:147-151)

Eine große Rolle spielt auch der Ersatz von Lebensmitteln, die emotional bedeutsam sind, durch andere, die die Bedürfnisse befriedigen.

Meine Familie und ich, wir lieben Eis. Das heißt, wenn ich mich da an die Fruchtsorten hineinlagere, wo nicht eben gerade Sahne oder sonst was drin ist, dann darf ich durchaus auch ein Eis essen [...] Mit Schokolade und Vanille und keine Ahnung, das muss ich nicht essen. Ich mag es auch sowieso nicht. Aber davon mal ganz abgesehen. Man hat also Möglichkeiten. Für mich war wichtig, Dinge auszutauschen. Einfach Ernährung, das, was nicht gut ist, versuchen, etwas zu finden, was ich auch mag, und das auszutauschen, was für mich vom Gesundheitsstandpunkt her besser ist. (P20:061)

P8 schildert eindrucksvoll die nervliche Belastung, aber auch die Erfolgsmomente, die mit dem Versuch zu verzichten einhergehen können. Was er sich wirklich wünscht, so wird im letzten Teil des folgenden Zitats deutlich, ist eine Speise, auf die er sich mit gutem Gewissen richtig freuen kann. Auf die positiven Emotionen, die Essen auslösen kann, möchte er nicht verzichten müssen.

Also, wenn Sie sich dann ne so 'ne Katze vorstellen, die um heißen Brei rumläuft, so komm ich mir manchmal vor, ich weiß dann, es gibt verschiedene Stellen, es gibt im Wohnzimmer 'ne Stelle, es gibt in der Küche 'ne Stelle und, meine Frau hat so 'n Nähzimmer, äh da gibt's auch 'ne Stelle, wo so was zu finden ist, wenn ich's suche (2) und, dann guck ich auch mal rein ((lacht)), ob da liegt, und denn, wenn man dann reingeguckt hat, naja und, man macht die Tür wieder zu und setzt sich wieder hin, und es sind so fünf Minuten vergangen, dann ist man richtig stolz, hast mal wieder geschafft, hast dich nicht verführen lassen. Mhm, kommt natürlich wie gesagt, bei der, bei dem Osterhasen ist es ja nun passiert [...]((räuspert sich)) Aber es muss was geben, wo man wirklich, wo man sich drauf freut (2) Und wenn es wie gesagt, wenn es Linsensuppe ist, ich esse gerne Linsensuppe (3) ja bald wissen Sie also mein ganzes Innenleben ((lächelt)). (P8:0661-0671)

In dieser Passage erzählt P8 sehr anschaulich, wie er sich durch den Wunsch nach bestimmten Genussmitteln in einen Konflikt gedrängt fühlt. Es ist wie eine Sucht; er fühlt sich wie ein Tier getrieben von seinem Instinkt, seine Bedürfnisse zu befriedigen. Dabei erweckt das Bild, das er verwendet, den Eindruck, dass er sich dabei auch roh, unzivilisiert fühlt. Dadurch, dass er verschiedene Stellen aufsucht, welche wie Verstecke über den ganzen Wohnraum verteilen, bekommt diese Szenerie eine „zwielichtige“ Atmosphäre, in der P8 sich möglicherweise wie ein Verbrecher vorkommt. Am Schluss dieser Episode hat P8 der Verführung widerstanden: Der gelungene Verzicht löst in ihm ein positives Gefühl des „Stolzes“ aus, verstärkt also sein Selbstbewusstsein und bringt sein Selbstbild mit den moralischen Ansprüchen überein. Diesem Erfolgserlebnis steht eine bereits erzählte Episode gegenüber, in der er, wie im Rausch, einen 
Schokoladenosterhasen verspeist hat. Diese Erinnerung löst einen Rechtfertigungsdruck aus und er erklärt das Recht auf Genuss zum Prinzip (,es muss was geben, [...] [auf das man sich] freut."). Er setzt noch nach, dass es sich bei einer seiner Vorlieben um Linsensuppe handelt, also nicht immer um Süßigkeiten, sondern auch um gesunde Nahrungsmittel. Damit stellt er sich als eigentlich genügsam dar und reagiert so auf eine hypothetische moralische Verurteilung. P20 ist es ebenfalls wichtig, befriedigenden Ersatz für frühere Genüsse zu finden:

Ja, also Senf unter Käse [statt Butter] schmeckt total lecker. Esse ich gerne. [...] Man muss so, so für sich selber so ein bisschen was finden, ähm, ja, wo man so sagt: "Guck mal, da habe ich mir das gespart." [...] Ähm, wo man sich so was spart und, und trotzdem auch zufrieden bei ist. Denn das ist ein un-, das ist im Übrigen ein unglaublich wichtiger Aspekt: dass man dabei auch ein Stück weit eine Zufriedenheit hat. Ähm, dass man [...] sich nicht nur kasteit. (P20:393)

Verzichten wird hier nicht als Arbeiten gegen, sondern für das eigene Wohlbefinden verstanden. In diesem Fall orientiert sich die Interviewte nicht an dem Erreichen bestimmter Werte, sondern die Erfüllung einer eigenen Regel selbst ist ein Erfolg. Damit wird Verzicht erträglich und ist sogar mit positiven Gefühlen verknüpft.

\subsubsection{Neue positive Erfahrungen machen}

Eigene Regeln können so gestaltet werden, dass sie erfüllbar sind, dass also erfolgreich verzichtet werden kann. Erfolgreiches Verzichten kann Wohlbefinden auslösen:

Bei uns in unserem Städtchen gibt es ein kleines Hallenbad, was das Frühschwimmen anbietet von halb sieben bis um acht. [...]. Und das mache ich jeden Donnerstagmorgen. [...] Kostet mich um diese Zeit natürlich auch oftmals Überwindung. Es ist kalt, es ist dunkel, es ist musselig. Aber dann denke ich mir immer: Komm überwinde mal Deinen inneren Schweinehund. Jetzt nichts wie ran. Wenn ich nach Hause komme, bin ich unglaublich stolz auf mich. Weil ich dann wieder ein Stückchen geschafft habe, was ich mei-, dass ich meinem Körper etwas Gutes tue. Und das habe ich in den Jahren zuvor, wie meine Kinder auch klein waren, da habe ich mich und meinen Körper vergessen. Und man muss einfach lernen, dass man auch für sich - das ist nicht immer leicht - so kleine Räume schafft, ja, wo man einfach sich besinnt und auch mal in sich hinein horcht. (P20:049-053)

Neben dem Stolz, die eigenen Widerstände besiegt zu haben, erfährt P20 große Befriedigung dadurch, etwas für sich selbst getan zu haben. Verzicht verschafft ihr einen Benefit. Die Lebensstiländerung wird häufig als sehr negativ empfunden, weil andere nicht verzichten müssen (vgl. Kap 5.3.2). Einigen Interviewten gelingt es, diese Pflicht zu normalisieren und so die negative Konnotation abzumildern.

Also ich esse das alles, äh, wie vorher auch. Aber im Prinzip soll ja auch ein gesunder Mensch eigentlich ein gewisses Maß an, also an ausgeglichener Ernährung zu sich nehmen. Also da, wenn man das vergleicht miteinander, dann ist das eigentlich identisch. Da ist kein Unterschied bei. (P22:066)

Und man lernt auch, danach sein Essen auszurichten. Also man, man lebt schon damit. Das ist richtig. Aber ich sage mir immer wieder, ich: "Okay, als gesunder Mensch sollte ich ja auch auf mein, äh, auf meine Ernährung 
achten." Und aus dem Grunde sehe ich das jetzt nicht, weil ich krank bin, dass ich das mache. Sondern ich sehe das eher all[es] positiv, äh, dass es gesünder ist. Dass es nicht unbedingt jetzt was mit dem Krankheitsbild zu tun hat. (P22:100)

Für P22 ist es eine Erleichterung, dass sie nicht die Einzige ist, die sich gesund ernähren soll. Bei P32 findet sich eine ganz ähnliche befreiende Erkenntnis in Bezug auf die sportliche Betätigung, die er immer abgelehnt hat.

Sport. Sport habe ich nicht betont. Ich habe mein Leben lang keinen Sport getrieben. Meine Herren. ((lacht)) Ich treibe jetzt Sport. Es ist nicht schlimm. Es ist wirklich nicht schlimm. (P32:270-274)

Und das ist dann das Positive an den [Schulungen]. Da habe ich viel-. Und dann hat sie uns auch an die Sportgelände der Uni geführt. Dann hat sie uns das alles gezeigt. [...] Und da bin ich vor allem in den Sommermonaten, wo es schön warm war, da war es voll, da waren viele Menschen. Dann sieht man auch andere Menschen. Sport: die tun das. Die tun es, weil sie es wollen. Keiner zwingt sie dazu. (P32:611)

Dass Menschen ohne Zwang Sport treiben, erlaubt es ihm möglicherweise, neben sekundären Motiven (um das Fortschreiten einer Erkrankung zu verhindern) auch aus reiner Freude sich körperlich zu betätigen. P16 gewinnt Verzicht ebenfalls etwas Positives ab. Er staunt über die Erfahrung, sich trotz Hunger und Sport wohlzufühlen.

Mir ist es also gut bekommen, das war also praktisch äh, neben dem Abnehmen auch das zweite Standbein dazu, aber muss man auch Disziplin haben [...]. Meine Frau [...] macht das also auch mit, [...] und wir gehen also jeden Abend, egal, was für Wetter ist, um achtzehn dreißig, neunzehn Uhr oder auch noch später eben halt unsere Runde [...] Wenn man's also macht, bekommt es einen also auch sehr gut [...] Man fühlt sich eigentlich dann [...], obwohl man Hunger hat [...], man fühlt sich [...] auch gut, man fühlt sich wohl, [...] man ist leistungsfähig. (P16:1680-1729)

Wichtig ist möglicherweise, dass er nicht allein verzichtet: seine Frau begleitet ihn. Auch P32 findet eine seiner religiösen Lebenswelt angepasste Form des Verzichtens, die keinen Ausschluss, sondern im Gegenteil eine noch intensivere Form der Gemeinschaft bedeutet - für ihn zugleich ein „Befreiungsschlag“: Gegen ärztlichen Rat, fastet er während des Ramadan, wobei er gleichzeitig an einem Diätproramm teilnimmt, bei dem er außer Trinknahrung nichts zu sich nehmen soll. Dass der Gesundheit zu schaden, eine Sünde für ihn ist, beeinflusst ihn sicherlich in Richtung seines neuen „gesunden“ Lebensstils. Die religiöse Gemeinschaft dürfte eine stark motivierende Kraft (gewesen) sein, in der „alle gleich“ sind und die er durch das Fasten intensiv erleben konnte.

Und der Monat war richtig schön, intensiv erlebt. Äh, abends gab es Essen, nicht für mich. Ich habe meine Beutel getrunken. Äh, es ist schon komisch, wenn alle essen und Sie da Ihren Shake haben und trinken. Aber glauben Sie mir, es ist wirklich, äh, herrlich gewesen. Es war, also, das war das beste Fastenmonat, die ich seit langem erlebt habe. Es war, haben Sie ja auch in der Moschee alles gesehen. Also auch da war ich ja intensiv mit einbezogen. Auch in der Küche war ich miteinbezogen. Ich habe geholfen, ich habe Essen zubereitet. Also, äh, man denkt, äh, wenn man jetzt, man kriegt Appetit. Überhaupt nicht. Sowieso, wenn ich faste, darf ich nicht essen. Das ist für mich verboten. Trinken, essen. Da wäre sowieso nichts gegangen. Auch abends dann, wo ich dürfte, ging wunderbar, 
diese Beu-, diese, äh, Trinknahrung, die hat den Appetit richtig-. Also der Körper hat alles gekriegt, war zufrieden. Wie gesagt, das war für mich das Ausschlaggebende, dieser Monat Fasten. Und für mich, nach der Überlieferung meiner Religion ist das Fasten, den Körper reinigen, den Körper gesund reinigen. [...] Also wie gesagt, das ist die Sache, was mich da auf diesen Trip gebracht hat: Ich kann es ändern. Ich kann es ändern, nicht Tabletten, nicht der Insulin, auch nicht der Arzt. Der ist nicht immer bei mir. Ich. (P32:348-352)

Für P32 stellt die Erkenntnis, von außen keinem Zwang zu unterliegen, einen Wendepunkt dar. Er äußert seine Freude darüber, dass es ihm sogar verboten ist, sich Dinge zu verbieten („Verbote sind verboten“, vgl. Kap. 5.6.4). Diese ungewöhnliche "Regel" ist viel leichter zu befolgen, da sie von außen bestätigt bzw. verhängt worden ist. Eine fast schon absurde, aber höchst produktive Steigerung dieser Verbotsregel schildert P32 in einer anderen Passage des Interviews. Zunächst will sein Arzt inm das Fasten während des Ramadans, also eine sehr konsequente Art zu verzichten verbieten:

Das muss ich dann nochmal erwähnen. Also das ist sehr wichtig. Zum Fasten, das war so: mein Hausarzt hat, habe ich mit inm darüber geredet, weil wir fasten einmal im Monat, einmal im Jahr einen Monat lang, von Sonnenaufgang bis Sonnenuntergang. Habe ich gesagt: "Darf ich das?" Kategorisch: „Nein, dürfen Sie nicht." - "Warum?" - "Sie sind Diabetiker. Sie haben Probleme." Fing alles an. Gut. Ein Jahr habe ich nicht gemacht. Das nächste darauffolgende Jahr, ohne den Arzt zu fragen, habe ich es gemacht. (P32:332)

P32 schildert gerade diesen gegen den Willen des Arztes durchgeführten Fastenmonat als Erfolg. In dieser Form gelingt ihm der Verzicht leichter als mit den bisherigen Diäten. Durch den Triumph, „heimlich“ auch noch die Werte zu verbessern, wird der Verzicht für inn selbstbestimmt, statt durch den Arzt bestimmt. Dass die Laborwerte dann die Richtigkeit seines Verhaltens „beweisen“, bestärkt ihn darin, von nun an selbst sein Verhalten zu bestimmen.

Nach diesem einen Monat [Ramadan] habe ich Blutuntersuchung gehabt bei meinem Hausarzt. [...] „Die Blutwerte sind jetzt auf 8,5, 8,7. Was ist passiert?" Da hat es bei mir 'klick' gemacht. Ich habe gefastet. Ich habe auch abgenommen gehabt in dieser Fastenzeit. [...] Also dass Fasten, dieser, dieser Monat, dieses Fasten hat mir gezeigt für mich, dass ich in der Hand habe, wie meine Werte sind. Davor habe ich gesagt: "Tja, die Tabletten machen das. Das Insulin macht das." Aber da habe ich es erst gesehen. Ich kann es machen. Das muss ich ehrlich, das. Wie gesagt, das war, nicht, äh, der Arzt kategorisch 'nein' gesagt. [...] Und dann kam dieses (Diät)Programm [...] Und wie der Zufall will, ist dieses Programm, dieses Beutelphase mit in die Fastenmonatszeit, äh, in Ramadan gekommen. Ich habe gefastet. Ich habe diesen einen Monat gefastet. Ich habe ganz normal die, diese Beutel, nennt sich das, weiter eingenommen. Und glauben Sie mir, in zwei Wochen sieben Kilo abnehmen, das muss mir jemand vormachen. Und da waren die Ärztinnen auch in diesem Bereich, bei diesem Programm sehr erstaunt. [...] Die Skepsis. "Sie dürften das eigentlich nicht mit Ihrem Diabetes." [...] [Im] Endeffekt hat sie nicht gesagt: "Sie dürfen es nicht [...]" Das ist dann mir überlassen worden. Alles andere hätte ich auch nicht akzeptiert. Weil das muss ich auch sagen. Weil ich kann mich in den Bereichen jetzt beurteilen, wie ich das mache. (P32:336-348)

Meistens sprechen Interviewte davon, mit dem Druck, ihr Leben ändern zu müssen, allein zu sein. Der soziale Ausschluss, der oft durch den Verzicht erlebt wird, scheint aber nicht immer negativ bewertet zu werden. Verzichten kann zu einer „Befreiung“ 
aus den familiären Rollenverpflichtungen führen. P32 beschert z.B. die Auflage, Sport zu treiben, ganz neue Momente für sich allein.

Da habe ich das auch gesehen: "Aha, es gibt auch was anderes als nur Arbeit, Haus, zu Hause sein, und, äh, die anderen." Abschalten, mal eine Stunde für sich. Das kannte ich nicht. (P32:433)

P20, die an anderer Stelle im Interview von ihren vielen Anforderungen als Mutter berichtet, gelingt es, sich eine Auszeit zu gönnen, um etwas für ihre Gesundheit zu tun.

Man muss ja sich so immer seine Nischen suchen. Da kann es mal sein - ich dusche nun schrecklich gerne abends - und dann habe ich mir die Kerzchen angemacht [...] Habe mich dann mit diesem Peeling mich super nett geduscht, im Kerzenschein, und habe dann gesagt: "Ich möchte jetzt meine Ruhe haben. Und hier kommt jetzt keiner rein mal eben Hände waschen oder irgendwas. Zehn Minuten." [...] Das ist auch ein unglaublich schönes Gefühl, mal so einen Moment ganz für sich zu haben. Und da sollte jeder versuchen, sich so, mal so zehn Minuten zu nehmen. Das braucht man auch manchmal um runter zu kommen. [...] Und so kann man sich auch mit ganzen Kleinigkeiten, ähm, etwas gönnen, was seiner Seele und dem Körper gut tut. (P20:162)

An anderer Stelle beschreibt sie eine weitere neue Angewohnheit, die sie sich „gönnt":

Aber diese Zeit [morgens], die nehme ich mir einfach ganz in Ruhe. Manchmal denke ich über den Tag nach. Manchmal hat man ja auch mal irgendwas, wo man so nachdenkt. Und, äh, ja, trinke ganz in Ruhe meine Tasse Tee und setze mich da ganz dekadent und frech hin und gönne mir einfach mal diesen Moment. Das sollte man vielleicht auch tun. Es tut einem unglaublich gut. Habe ich jetzt festgestellt. Mir tut es gut und ich werde es noch eine Weile so machen. Schauen wir mal, was mir dann wieder einfällt. (P20:156)

Sie erzählt, wie sie sich Zeit für sich nimmt, in Ruhe einen Tee trinkt und bezeichnet dies als "dekadent“. Dadurch unterstreicht sie, wie wenig sie sich sonst im Alltag zugesteht und stellt sich so als bescheiden dar. Möglicherweise hat sie erst durch den Druck, ihr Leben umzustellen und zu verzichten, diese „Befreiung“ entdeckt. Um sich etwas Gutes zu tun, was aus ihrer Sicht auch zu einem gesunden Leben gehört, "gönnt" sie sich etwas, nämlich Ruhe, ähnlich wie P32, der Sport treibt. Dabei wird ihr eigentliches Bild von Erwartungen auf den Kopf gestellt: Eigentlich soll sie sich doch gerade nichts gönnen, sondern verzichten. So beschreibt sie dieses Vorgehen als „dekadent", merkt jedoch, wie „richtig“ es ist („das sollte man vielleicht auch tun“) und ihrer Gesundheit guttut.

Die hier genannten Erfahrungen mit Verzicht sind sehr unterschiedlich, aber dennoch positiv und ein alternatives Muster zum bisherigen Leben. 


\section{Diskussion}

Verzicht ist die vorherrschende bzw. beherrschende Erfahrung der interviewten Menschen mit Diabetes Typ 2. Alle stehen unter dem Druck, auf ein genussvolles Leben verzichten zu müssen, was den meisten ungerecht und schmerzhaft erscheint. Ob ihr Verzicht in der Gegenwart ausreicht bzw. in der Vergangenheit ausreichte, versuchen sie anhand von „sichtbaren" Erfolgskriterien zu bewerten. Körpermaße und Laborwerte - vermeintlich unmittelbare Ergebnisse von Verzicht oder seinem Gegenpart Maßlosigkeit - dienen als moralische Prüfmarke im Rahmen von medizinischen Konsultationen, aber auch im privaten sozialen Leben und innerhalb der Selbstreflexion und -wahrnehmung. Aus Angst vor moralischer Diskreditierung unternehmen alle Interviewten großen Aufwand, sich von Maßlosigkeit abzugrenzen. Viele beklagen, dass ihre „unsichtbaren“ Bemühungen nicht anerkannt würden. Wenn sie auch gegen die "oberflächliche" Bewertung und Körpernormierung opponieren, so können sie sich dem Druck, dass von ihrem Äußeren auf ihr Verhalten geschlossen und daraufhin ihr Charakter bewertet wird, nicht entziehen. Diese (innere) Verhandlung von Schuld und Moral setzt sich auch in den Konzepten oder „Regeln“ fort, die (unbewusst) notwendigerweise alle Interviewten zur praktischen Orientierung für den Verzicht entwickeln. Auffällig ist eine den Dualismus von Verzicht und Maßlosigkeit aufgreifende Einteilung in "gesunde" und "ungesunde" Lebensmittel. Das kann zu schmerzhaften Konflikten und Unsicherheit bei täglichen Entscheidungen führen. Gleichzeitig ergibt sich aber wiederum eine gewisse Freiheit, emotional bedeutsame Lebensmittel oder Verhaltensweisen als gesund oder relativ zu anderen Genüssen als gesünder zu definieren, sodass die „Regeln“ zu den eigenen Bedürfnissen und Fähigkeiten passen. Das Erfüllen der eigenen „Verzichts-Regeln“ und das Erhöhen des eigenen Wohlbefindens durch neue Gewohnheiten dienen als „unsichtbare“ persönliche Erfolgskriterien. Manchmal führt Verzicht dadurch zu positiven Erfahrungen verliert damit seine negative Konnotation.

\subsection{Stärken und Schwächen}

Ziel der vorliegenden Arbeit war die Untersuchung von Krankheitserfahrungen von Menschen mit Diabetes Typ 2 mittels eines qualitativen Verfahrens, der Grounded 
Theory. Steinke (1999) formulierte zur Beurteilung der Güte qualitativer Forschung sieben Bewertungskriterien. Dazu gehört ihre Forderung nach einer Indikation des Forschungsprozesses. In der Annahme, dass die hier untersuchten lebensweltlichen Erfahrungen in ihrer Komplexität die Möglichkeiten quantitativer Forschung übersteigen, wurde in der vorliegenden Arbeit bewusst ein qualitativer, interpretativer Forschungsansatz gewählt.

Als Material lagen insgesamt 35 bereits für das Website-Projekts „krankheitserfahrungen.de" erhobene narrative Interviews vor. Die Wahl der Interviewpartnerinnen und -partner folgte dem Maximum Variation Sampling (s. S. 38). Jedes Interview wurde durch eine offene Eingangsfrage eingeleitet ${ }^{19}$, um die Befragten anzuregen, alle aus ihrer Sicht relevanten Erfahrungen zu berichten. So erfülte das umfangreiche Ausgansmaterial sehr gute Voraussetzungen für eine weitere, hier vorliegende Analyse mittels Grounded Theory, für die ein offener Zugang von großer Bedeutung ist. Dieses Ausgangsmaterial erlaubte dann eine - im Sinne eines Theoretical Samplings - analysegeleitete Auswahl von Ausschnitten aus 14 Interviews und deren Interpretation.

Strauss formulierte mehrere Hauptelemente einer Studie nach der Grounded Theory (Strauss 1998; Legewie und Schervier-Legewie 2004). Dazu gehört das Theoretical Sampling, bei dem analysegeleitet Daten in die Untersuchung einbezogen werden, um die Erkenntnisse immer weiter zu verdichten. Mithilfe des Theoretical Samplings ist es möglich, eine geringe Anzahl an Fällen gezielt tiefergehend zu untersuchen. Genau deshalb fordert Steinke (1999) die Auseinandersetzung mit der Limitation einer qualitativen Studie. Weil die Stichprobe viel zu klein ist, um Menschen mit Diabetes Typ 2 in Deutschland zu repräsentieren, ist es natürlich nur begrenzt möglich, die Ergebnisse zu verallgemeinern. Fallübergreifend ist es aber gelungen, wiederkehrende Deutungsmuster bzw. Krankheitskonzepte zu identifizieren, bei denen Verzicht und Maßlosigkeit eine bedeutende Rolle spielen. Nicht auszuschließen ist, dass es Menschen mit Diabetes Typ 2 gibt, die Verzicht nicht als beherrschend empfinden. Was die Studie leisten kann, ist für die Lebenswelt und etwaige Schwierigkeiten in der (Selbst)Behandlung

\footnotetext{
19 „[...] Ich möchte Sie nun bitten, mir von Ihren Erfahrungen zu erzählen, vielleicht von dem Zeitpunkt an, als Sie das erste Mal das Gefühl hatten, dass irgendetwas nicht in Ordnung ist, oder als der Diabetes bei Ihnen festgestellt wurde." (s. a. S. 39)
} 
dieser Menschen zu sensibilisieren. Damit ist das von Steinke (1999) vorgeschlagene Kriterium der Relevanz der Ergebnisse für die Praxis gegeben.

Neben dem Theoretischen Sampling fordert Strauss ein Theoretisches Kodieren (Strauss 1998, S. 51; Legewie und Schervier-Legewie 2004), um Konzepte innerhalb der Erzählungen zu finden und zu benennen, gemeinsam mit einem kontinuierlichem Vergleichen während des Analyseprozesses (Constant Comparative Method) - ein drittes wichtiges Element der Grounded Theory (Strauss 1998; Legewie und SchervierLegewie 2004). Die Integration der durch das Kodieren gesammelten Konzepte und weiteren Datenmaterials erlaubte es dann, Kategorien zu entwickeln, die theoretisch betrachtet werden können, aber in den Daten gegründet (grounded) sind. Dies entspricht Steinkes (1999) Forderung nach einer empirischen Verankerung der Erkenntnisse. Ein kohärentes Theoriemodell mit Darstellung der verschiedenen Ausprägungen der Erfahrungen anhand von Textbelegen, die vergleichend gegenübergestellt wurden, war das Ziel.

Um eine intersubjektive Nachvollziehbarkeit der Studienergebnisse zu erreichen, d.h., „einem externen Publikum die Möglichkeit [zu] [...] geben, die Untersuchung Schritt für Schritt zu verfolgen und den Forschungsprozess sowie die daraus hervorgegangenen Erkenntnisse zu bewerten", soll der Forschungsprozess laut Steinke (1999, S. 209) möglichst ausführlich dokumentiert werden. Die vorliegende Arbeit zeigt anhand konkreter Beispiele das Vorgehen beim Kodieren und beim Sampling. Ausschnitte aus den Memos sollten zeigen, wie ich zu theoretischen Überlegungen angeregt wurde. Die Darstellung der Ergebnisse erfolgte durch Gegenüberstellung von Zitaten, d.h. „rohen" Daten und den jeweiligen Interpretationen und abgeleiteten Hypothesen, um den Auswertungsprozess verständlich und transparent zu machen. Die Nachvollziehbarkeit wurde während der Analyse durch Vorstellung der Ergebnisse bei Treffen und Kolloquien im Institut immer wieder überprüft.

Hilfreich war die Anwendung des computergestützten Kodier-Programms Atlas.ti (Atlas.ti $\mathrm{GmbH}$ ) für eine systematische Kodierung mit der Möglichkeit des schnellen Zugriffs auf die unter einem Kodenamen versammelten Daten.

Sicherlich wäre eine ausführlichere Dokumentation der eigenen Vorannahmen sinnvoll gewesen, womit das Kriterium der reflektierten Subjektivität noch stärker erfüllt wäre. 
Durch die Erhöhung der für die Forschung nach der Grounded Theory notwendigen Theoretischen Sensibilität hat die Forscherin in ihrer Rolle als angehende Ärztin aber versucht, die Erzählungen sowohl mit Distanz als auch mit empathischer Aufmerksamkeit zu betrachten. ${ }^{20}$

Wäre die Datenerhebung selbst Teil meiner Arbeit gewesen, wäre das Theoretische Sampling noch besser "datengeleitet" möglich gewesen. So hätten ganz neue „Fälle“ mit anderen Bedingungen gesucht werden können, z.B. Teenager mit Diabetes Typ 2 und Adipositas oder Menschen mit Diabetes Typ 2 und einer Essstörung oder in psychologischer bzw. psychiatrischer Behandlung wegen einer Depression.

\subsection{Verzicht: zwischen Entbehrung und moralischer Läuterung}

"Verzichten" wurde als Wort von mehreren Interviewpartnern verwendet und beherrschte in der einen oder anderen Form bei allen die Krankheitserfahrung mit Diabetes Typ 2. An dieser Stelle soll zunächst die allgemeinsprachliche Bedeutung des Begriffs Verzicht diskutiert werden, um für seine Bedeutung in der Krankheitserfahrung zu sensibilisieren. In den folgenden Kapiteln wird seine Angemessenheit als Kernkategorie diskutiert.

Im Duden sind folgende Synonyme für verzichten angeführt: „abschwören, absehen von, aufgeben [...], sich enthalten (geh. [gehoben]), entsagen (geh.)" (2002, S. 1007; Stichwort verzichten). Bei Betrachtung dieser Wörter wird eines deutlich: Verzicht heißt etwas „aufgeben“, also ohne etwas auskommen müssen, einen Mangel an etwas haben. „Entsagen“, „sich enthalten“ und „abschwören“ weisen darauf hin, dass Verzicht einer kognitiven und emotionalen Abgrenzung von dem Gegenstand bedarf, auf den verzichtet werden soll. Eine Impulskontrolle und Selbstbeherrschung ist notwendig. Bei den Gegenständen, auf die verzichtet wird, handelt es sich um emotional bedeutsame Objekte: Genussmittel wie bestimmte Speisen, Alkohol, Zigaretten, Geld. In der Rechtsprechung bezieht sich Verzicht auf persönliche Vorteile, die nicht in Anspruch genommen werden.

Verzicht ist meist subjektiv mit einer gewissen Anstrengung oder Schmerz assoziiert. Gleichzeitig ist mit Verzicht in der Regel ein Ziel, eine positive Aussicht verbunden.

20 Vgl. Kap. 4.4.3 
Dadurch hat Verzicht eine ambivalente Bedeutung. Gründe, auf etwas zu verzichten, sind beispielsweise der Wunsch, die eigene Gesundheit zu fördern, Krankheiten vorzubeugen oder deren Fortschreiten zu verzögern. Bei Verzicht auf Genussmittel oder generelle Kalorienreduktion spielen Schönheitsideale eine große Rolle. Meist ist der Vorteil, der vom Verzicht erwartet wird, nicht unmittelbar verfügbar, sondern mit einer gewissen zeitlichen Latenz und Ungewissheit verbunden. So ist z.B. der Effekt durch Verzicht auf einen Schokoladenriegel oder die Entscheidung gegen ein anderes Genussmittel in der Regel nicht sofort im Anschluss daran ersichtlich. Erst nach einigen Tagen bis Wochen wird sich eine Wirkung z.B. Gewichtsreduktion zeigen, möglicherweise aber auch nicht. Weiterhin wird Verzicht als Übung der Selbstkontrolle im Sinne geistiger Reinigung oder religiöser Läuterung verstanden, z.B. beim Fasten. Seit dem Altertum ist die Askese als Methode der Klärung des Geistes durch Überwindung und Befreiung von „Begierden“ bekannt (Röcke und Weitbrecht 2010).

Verzicht auf persönliche Ansprüche kann moralische Erhöhung und Überlegenheit über Mitmenschen bedeuten. Verzicht auf gesundheitsschädigendes Verhalten ist im Sinne von Prävention zu einem selbstverständlichen und verpflichtenden Teil der Medizin geworden: „Vorbeugung avanciert zur moralischen Pflicht“ (Bröckling 2008). Das Rauchen aufgeben, Sport treiben und insbesondere Mäßigung beim Essen sind omnipräsent in der Gesundheitsvorsorge. „Völlerei“ oder "Maßlosigkeit im Essen und Trinken" sind auch heute in einer weltlich ausgerichteten Gesellschaft noch in der Bedeutung von "Sünde“ von hoher Aktualität (Bucher 2012). Wie sich in der vorliegenden Analyse zeigte, ist „Verzicht" als Gegenpart zu „Maßlosigkeit“ bei Diabetes ein beherrschendes und auf spezifische Weise verhandeltes Thema.

\subsection{Verzicht bei Diabetes Typ 2 - eine fast ungerechte Forderung}

Der Druck, nach der Diagnose plötzlich auf frühere Genüsse verzichten zu müssen erscheint den Interviewten schmerzhaft, "hart", wie es eine Interviewte beschreibt. „Harte“ Regeln, „hart“ sein müssen - das scheint einem lebendigen Alltag zu widersprechen. Die meisten Interviewten empfinden die (ärztliche) Aufforderung, ihren Lebensstil zu verändern, als pauschale, diffuse Forderung auf alle und gerade innen emotional wichtigen Genüsse zu verzichten. Sie fühlen sich gegenüber Menschen ohne Diabetes Typ 2 benachteiligt und ungerecht behandelt: wenn z.B. Menschen, die 
„beleibter" $\operatorname{sind}{ }^{21}$, offensichtlich einen ungesünderen Lebensstil als sie betrieben haben, aber nicht verzichten müssen, weil sie gute Laborwerte haben. Es scheint den Interviewten, als dürtten andere sich auf eine Weise verhalten, die bei innen selbst als „maßlos“ tituliert und als ursächlich für Diabetes (und Übergewicht) gelten würde.

Viele klagen darüber, im Gegensatz zu anderen nicht mehr unbeschwert essen und trinken zu können. P21 hat das Gefühl, nicht mehr „frei“ zu sein"22, sondern die ganze Zeit daran denken zu müssen. Viele Interviewte fühlen sich von sozialen Anlässen, die von gemeinsamem Essen geprägt sind, ausgeschlossen oder glauben sich aus Schutz vor Verführungen ausschließen zu müssen.

Während Diabetes selbst nicht zu spüren ist, bringt Verzicht schmerzhafte Empfindungen mit sich, z.B. „permanent Hunger“ zu haben ${ }^{23}$. Die Krankheit setzt keine äußere Grenze, sondern die Interviewten müssen sich selbst Grenzen auferlegen. Statt wie bei anderen Krankheiten die Wiederherstellung des eigenen Wohlbefindens zum Ziel zu bestimmen, sollen sie gegen ihre Bedürfnisse ankämpfen. Weiterhin fühlen sich mehrere Interviewte allein gelassen. Sie erleben die ärztliche Behandlung als reduziert auf die Aufforderung zu verzichten. Insgesamt hat also Verzicht für die Interviewten zunächst einmal eine negative Bedeutung.

Wenn auch nicht mit dem Begriff Verzicht bezeichnet, hat der als schmerzhaft erlebte Druck, den Lebensstil zu verändern, bereits einige Beachtung in der Forschungsliteratur zu Diabetes Typ 2 gefunden. Wesentliches Ergebnis der qualitativen Analyse von Ahlin and Billhult (2012) ist, dass die von ihnen in Schweden interviewten zehn Frauen mit Diabetes Typ 2 den Druck, den Lebensstil zu verändern, als ununterbrochenen innerlichen und äußeren Kampf („continuous struggle“) empfanden. Sie fühlten sich als Opfer der Krankheit und unfair vom Leben behandelt, geradezu ihrer Lebensfreude beraubt und leer. Auch in der hier vorliegenden Analyse hatten viele Interviewte das Gefühl, dass ihnen ein ihnen zustehender Genuss vorenthalten würde. Beispielhaft sei P21 erwähnt, die sich im Alter auf Verabredungen zu Kaffee und Kuchen gefreut hat. Diesem Vergnügen soll sie nun entsagen, während es anderen erlaubt ist zu genießen.

\footnotetext{
${ }^{21} \mathrm{vgl}$. Zitat (P21:602), S. 68

22 vgl. Zitat (P21:318), S. 52

${ }^{23}$ vgl. Zitat (P16:1226), S. 58
} 
Yannakoulia (2006) weist in einem Review zu Ernährungsgewohnheiten bei Diabetes Typ 2 darauf hin, dass Betroffene sich häufig durch die Diätempfehlungen eingeengt fühlen und diese als nicht zu ihren Bedürfnissen passend erleben. Besonders zu Beginn der Ernährungsumstellung verfolgen viele Erkrankte den Anspruch, sehr restriktive Diäten einzuhalten, z.B. Zucker vollkommen zu vermeiden. Der große Druck, sich gesund zu ernähren, kann dazu führen, dass sie nicht ehrlich über ihre Ernährungsgewohnheiten sprechen, nur über den Verzehr von „erwünschten“ Lebensmitteln berichten und Schwierigkeiten verschweigen. Der Druck kann auch ein Gefühl der Gleichgültigkeit gegenüber den Verhaltensempfehlungen verursachen. Yannakoulias Ausarbeitung stützt unser zentrales Ergebnis: dass nämlich der Begriff „Verzicht“ angemessen die von Menschen mit Diabetes Typ 2 als restriktiv erlebte Lebensstilumstellung beschreibt.

Péres et al. (2008) berichten von starken Gefühlen der Angst und Wut, die Frauen in Brasilien kurz nach der Diagnose von Diabetes Typ 2 empfanden. Sie vermuten, dass dies besonders durch den Eindruck, „alles“ sei verboten, erzeugt wird. Diese emotionalen Reaktionen finden sich auch in den Ergebnissen der vorliegenden Studie, z.B. bei P2, der kritisiert, dass inm „alles verboten“ wird. ${ }^{24}$ Ähnliches entdeckten Whittemore et al. (2002) in ihrer qualitativen Untersuchung im Rahmen eines Interventionsprogramms mit dem Ziel, zu einem „besseren“ Selbstmanagement bei Diabetes Typ 2 zu motivieren. Besonders das Streben nach Befriedigung der eigenen Bedürfnisse („Striving for satisfaction“) erwies sich dabei als besondere Herausforderung bei der Integration von Diätmaßnahmen in die frühere Lebensweise. So äußerten die von innen in den USA interviewten neun Frauen, einigen Personen unserer Studie ähnlich, das Gefühl, bei Einhaltung der Diätempfehlungen nicht richtig satt zu werden und die Umstellung des Lebens daher als zu drastisch und gar unmenschlich zu empfinden. Die hier vorliegenden Ergebnisse weisen außerdem auf das Dilemma hin, dass Diabetes (zunächst) keine spürbaren Nachteile bietet, der Verzicht jedoch umso schmerzlicher spürbar ist. Verzicht ist schwer zu ertragen, da dem eigenen Verhalten von auBen keine Grenzen gesetzt sind, z.B. durch Schmerzen oder durch erzwungene Bettlägerigkeit, wie z.B. nach einer Operation ${ }^{25}$, sondern die Personen aufgefordert sind,

${ }^{24} \mathrm{Vgl}$. Zitat (P2:055), S. 59

25 Vgl. Zitat (P2:029), S. 56 
aus eigener Kraft ihren Genüssen zu widerstehen, sich zu „quälen“, um sich langfristig vor Komplikationen zu schützen. Dies ergänzt die Überlegungen von Whittemore et al. (2002), die den Mangel an Symptomen als Hindernis für die Umstellung der Lebensweise erkannten, so dass sich Erkrankte nicht persönlich durch den Diabetes gefährdet fühlen.

Vorstellbar ist, dass Verzicht bei Diabetes Typ 2 deshalb als besonders schmerzhaft erlebt wird, weil die Forderung ungerecht erscheint, gleichzeitig aber ein hoher Anpassungsdruck durch die moralische Bewertung von Verzicht besteht. Beispielhaft möchte ich noch einmal die Frage erwähnen, um die P30 kreist: „Was dürfte ich?"26. Dieser Ausspruch eröffnet den Blick auf einen interessanten Zusammenhang: P30 erlebt, dass sie laut einer äußeren Autorität oder einem moralischen Gebot nicht alles "darf“, gleichzeitig wird sie aber im Unklaren darüber gelassen, was genau ihr verboten ist. Alle Interviewten scheinen sich zerrissen zu fühlen zwischen dem Gefühl der Ungerechtigkeit, im Gegensatz zu ihren Mitmenschen verzichten zu müssen, die wie ein Rechtsentzug, also wie eine Strafe erlebt wird (vgl. Ahlin and Billhult 2012), und der äußeren Erwartung, sich als maßvoll darzustellen und die von innen erwartete Motivation zu beweisen. Möglicherweise kommt es Menschen mit Diabetes Typ 2 so vor, als ob bei innen wegen der Diagnose Diabetes ein anderes und höheres moralisches Maß angelegt wird als bei den Mitmenschen. Woher kommt die moralische Bewertung, die das Erleben von Verzicht maßgeblich bestimmt?

\subsection{Moralisierung von Lebensstil, Körpermaßen, Krankheit}

Wenn Interviewte von ärztlichen Kontrollterminen erzählen, bei denen sie ihre Laborwerte erfahren, wird eine gewisse Anspannung oder Nervosität deutlich ${ }^{27}$. Diese lässt sich dadurch erklären, dass die Interviewten die Beurteilung der Laborwerte als Beurteilung ihres Verhaltens wahrnehmen ${ }^{28}$ und als Urteil über ihre Person: die einen werden zum „Musterpatient“, die anderen erwarten das „Todesurteil“. ${ }^{29}$ Die Laborbefunde werden beinahe wie ein Zeugnis über das gute oder schlechte Verhalten verhandelt, als Zeichen von gelungenem „Verzicht“ bzw. seinem Gegenpart „Maßlosigkeit“. Alle

\footnotetext{
${ }^{26}$ Vgl. Zitat (P30:248), S. 54

27 Vgl. Zitate (P21:570), S. 61 und (P1:545), S. 62

28 Vgl. Zitat (P21:570), S. 61

29 Vgl. Zitate (P16:1482), S. 62 und (P1:545), S. 62
} 
Menschen mit Diabetes Typ 2, d. h. mit nicht der Norm entsprechenden Blutzuckerspiegeln, können sich mit dem Vorwurf konfrontiert sehen, maßlos gelebt zu haben und letztlich faul zu sein. Anzunehmen ist, dass sich daraus ein gewisses Abhängigkeitsgefühl vom ärztlichen Urteil ergibt. Diese (unbeabsichtigte) Abhängigkeit erschwert - selbst im Zeitalter aufgeklärter und partnerschaftlicher Medizin (Himmel und Rönsberg 2012) - die Gleichberechtigung zwischen Menschen mit Diabetes und ärztlichem Personal; in der Beziehung wird ein deutliches Machtgefälle erlebt. Vergleichend möchte ich diesem Muster beispielhaft die Befundmitteilung bei einer Brustkrebserkrankung gegenüberstellen. Haben sich in einem solchen Fall die Laborbefunde verschlechtert, wird der Arzt oder die Ärztin die betroffene Person keineswegs „wieder fertigmachen“ 30 oder sie als „Musterpatient“ loben.

Die Beurteilung der Laborwerte wird zu einem emotionalen Akt und prägt die ArztPatient-Beziehung stark. Werden Körpermaße bewertet, ist dieses Muster noch deutlicher spürbar. Alle Personen erzählen auf die Frage nach ihren Erfahrungen mit Diabetes Typ 2 ausführlich über die Themen Körpermaße und Gewicht, manche sogar unmittelbar zu Beginn des Interviews. ${ }^{31}$ Dies entsprach insofern der Erwartung, als dass Diabetes Typ 2 als „Krankheit der Übergewichtigen“ gilt (Bucher 2012). Bemerkenswert war dennoch, wie stark letztlich alle Interviews das Konzept einer stabilen Einteilung der Gesellschaft in Dicke und Dünne bestätigten, in der Abstufungen kaum Platz haben. Alle Interviewten stehen unter Druck, das eigene Über- oder Normalgewicht zu erklären. Kein Wunder, dass ein großer Aufwand betrieben wird - und zwar auch von denjenigen, die nie Gewichtsprobleme hatten -, sich von „schlechten“ Eigenschaften abzugrenzen. Viele Interviewte vergleichen sich mit anderen Menschen, die sie durch die Beschreibung ihres Verhaltens als maßlos charakterisieren und stärken durch diese Abgrenzung inr Selbstbewusstsein. ${ }^{32}$ Mit dieser Abgrenzung reproduzieren sie die strenge Einteilung in dick und dünn, maßlos und genügsam.

Hier lässt sich anknüpfen an einen Review über Stigma bei Diabetes, aber auch bei Hepatitis C, HIV, Epilepsie, Adipositas und Zöliakie, die jeweils in der öffentlichen

\footnotetext{
30 Vgl. Zitat (P1:545), S. 62

${ }^{31}$ Vgl. (P6:010-013): Interviewer: „[...] Ich werde Sie erst einmal nicht unterbrechen und einfach zuhören. “ P6: „Also, Ende der achtziger Jahre ist bei mir - damals noch 'ne leichte - Diabetes festgestellt worden, durch, ich will dazu sagen extremes Übergewicht."

32 Vgl. Zitate (P1:521), S.68 und (P21:602), S. 68
} 
Wahrnehmung ähnliche Merkmale, z.B. die Annahme von Verhalten als ursächlichem Faktor und diätetische Maßnahmen in der Behandlung haben: Schabert et al. (2013) vermuten, dass Diabetes wie Adipositas eine Krankheit sei, für die Menschen oft schuldig gesprochen würden. Interessant ist auch ihr Hinweis auf eine Studie zu Erfahrungen mit Hepatitis C (Butt et al. 2007): die allgegenwärtig erlebte Assoziation mit Drogenabhängigen bringe Menschen mit dieser Krankheit dazu, sich entschieden von intravenös applizierten Drogen abzugrenzen. Dies ähnelt den Ergebnissen unserer Studie, in denen Interviewte sich nachdrücklich von Übergewicht abgrenzen wollten.

Viele der Interviewten kritisieren zwar die Diskriminierung von Übergewichtigen, stellen aber ebenfalls einen Kausalzusammenhang zwischen Diabetes und Übergewicht her, z.B. P3, der einen Bogen von den Provokationen, denen Übergewichtige ausgesetzt sind, über reaktives Mehr-Essen („Frustessen“) bis zum „Endergebnis Diabetes“ spannt. ${ }^{33}$ Puhl et al. (2007) untersuchten mithilfe eines Onlinefragebogens den Zusammenhang zwischen internalisierten negativen Vorurteilen gegenüber Adipösen und dem Essverhalten von Menschen mit Übergewicht. Befragt wurden Frauen in den USA, die in einer nichtkommerziellen Organisation zur Gewichtsabnahme Mitglied waren. Teilnehmerinnen, die negative Stereotype über Adipöse, wie "faul“, „isst zu viel“ und „nicht intelligent", für wahr hielten, berichteten häufiger über eigenes Binge-Eating als Frauen, die die Stigmatisierung nicht internalisiert hatten. Deshalb sei die gängige Vorstellung anzuzweifeln, dass Stigmatisierung Menschen motivieren könnte, abzunehmen. Im Gegenteil scheint die Selbst-Stigmatisierung sogar einen gegenteiligen Effekt zu haben, worüber sich gerade medizinisches Personal klar werden müsse.

Aus dem Aufwand, den die Interviewten unserer Studie zur Abgrenzung von den Maßlosen betrieben, lässt sich ablesen, wie mächtig die Verknüpfung von Diabetes und Übergewicht verinnerlicht ist, genauso wie die Angst, auf die monokausale Erklärung "Übergewicht“ reduziert und moralisch diskreditiert zu werden. Genährt wird diese Angst durch die einseitige mediale Präsentation der Ursachen von Diabetes. So untersuchten beispielsweise Gollust und Lantz (2009) 698 zwischen 2005 und 2006 publizierten Artikel zum Thema Diabetes in 19 US-amerikanischen Zeitungen: Indivi-

${ }^{33}$ Vgl. Zitat (P3:154), S. 70 
duelle Eigenverantwortlichkeit wurde sowohl als Ursache für Diabetes als auch als Behandlungsstrategie in der Mehrzahl der Artikel genannt, während sich nur eine geringe Zahl an Artikeln mit den sozialen Umständen der Krankheitsentstehung und sozialpolitischen Ansätzen zur Lösung des Problems auseinandersetzte, obwohl die überproportional höhere Prävalenz bei sozial benachteiligten Bevölkerungsminderheiten bekannt ist (Mokdad et al. 2001; Mendenhall et al. 2010).

McNaughton (2013) thematisierte für Australien das Phänomen, dass seit Ende der 1990er Jahre die Annahme, Übergewicht sei vorrangige Ursache oder Risikofaktor bei Diabetes, sowohl in Laiendiskursen als auch in der Gesundheitspolitik allgegenwärtig sei, obwohl es viele weitere Risikofaktoren gäbe und ihr Zusammenspiel nicht eindeutig geklärt sei. Stattdessen werden mittlerweile Übergewicht und Diabetes Typ 2 in der öffentlichen Vorstellung sogar vermischt: zu dem Begriff "Diabesity“. Australische Publikationen von 1998 bis 2013, die McNaughton zu den Stichwörtern "diabesity“, „obesity risk diabetes“ ",obesity causes diabetes“ und „weight or fat causes diabetes" fand, umfassten Gesundheitsberichte aus Politik und von NGOs, Zeitungsartikel und Medienkommentare und wurden von der Autorin untersucht. Dabei fiel auf, dass Übergewicht und Diabetes in der öffentlichen Meinung in dem untersuchten Zeitraum zunehmend zu einem Gesundheitsproblem verschmolzen, z.B. wenn in Studien sprachlich immer häufiger suggeriert wurde, dass Übergewicht Diabetes verursacht, ohne den gegenteiligen Wirkungszusammenhang zuzulassen, dass Übergewicht auch Symptom von Diabetes sein könnte. Für die Autorin ist insbesondere die „AusDiab Study - 2000“ (Dunstan et al. 2001) sprachlich ungenau und tendenziös, was das Wissen über die Ursachen von Diabetes und Übergewicht als Epidemie betrifft. McNaughton argumentiert, dass unbeeinflussbare Risikofaktoren wie Gene oder Armut viel weniger in der Öffentlichkeit bedacht werden und Übergewicht und Diabetes dadurch zu primär selbstverursachten Risikofaktoren und Krankheiten moralisiert werden. Dies kann schwerwiegenden Einfluss auf die Krankheitserfahrung haben und zu Stigmatisierung führen. Rufe danach, Menschen durch Beschämung und Stigmatisierung dazu zu bringen, einen ungesunden Lebensstil zu vermeiden, lehnt McNaughton ab, da Stigmatisierung nicht auf ethisch korrekte Weise eingesetzt werden kann (s.a. Burris 2008). 
Ohne unterstellen zu wollen, dass dies Alltag in ärztlichen Praxen ist, fielen einige Erzählungen ins Auge, in denen Interviewte von sehr kränkenden Erfahrungen sprechen, wenn medizinisches Personal (ungefragt) ihre Körpermaße kommentierte. Drei Interviewte schildern ähnliche Begebenheiten, in denen sie sich schutzlos und ohne Vorwarnung einer solchen Beurteilung ausgesetzt fühlten. ${ }^{34}$ Die moralische Abwertung bei Übergewicht hat ein identitätsschädigendes Potential. Wenn sich Menschen nicht einmal mit den üblichen Höflichkeitsformeln behandelt, sondern „beschimpft“, in ihrer Ehre angegriffen fühlen, dürfte sich das negativ auf die Arzt-Patient-Beziehung und die Fähigkeit zur Krankheitsbewältigung auswirken. Der Druck zu verzichten wird vielleicht erhöht; es ist aber schwer vorstellbar, dass eine solche Interaktion zur selbstbestimmten Lebensstiländerung motiviert.

Die moralische Bewertung von Verzicht und Maßlosigkeit führt zu einem großen Druck, sich als genügsam darzustellen - nicht zu denen zu gehören, die die Erwartungen nicht erfüllen. Es überrascht nicht, dass auch Schönheitsideale in den Interviews zu Erfahrungen mit Diabetes Typ 2 zur Sprache kommen, die als entscheidend für sozialen Erfolg wahrgenommen werden und von denen die Interviewten sich, auch wenn sie diese Oberflächlichkeit der Gesellschaft ablehnen, nicht befreien können. Beispielhaft nennen möchte ich P3, der bei einer Diätberatung über die Angestellte denkt, „wie guckt die mich denn gerade an, der [...] ihr Typ bin ich wohl nicht“. ${ }^{35}$ Bei aller Ironie scheinen sich darin auch Ängste vor persönlicher Ablehnung durch medizinisches Personal und durch das alltägliche soziale Umfeld auszudrücken.

Teixeira und Budd (2010) zufolge kann eine mit Übergewicht einhergehende Stigmatisierung die Behandlung des Übergewichts und auch des Diabetes erschweren. Ein Großteil der Gesellschaft, einschließlich Ärztinnen und Ärzte, teilweise sogar jene, die sich auf Übergewicht spezialisiert haben, sind gegenüber übergewichtigen Menschen negativ eingestellt (Poon und Tarrant 2009; Schwartz et al. 2003; Teachman and Brownell 2001). Miller et al. (2013) beschrieben, wie ein „anti-obesity bias“ schon Medizinstudierende betreffe, sie sich dessen aber allzu selten bewusst seien. Foster et al. (2003) deckten in einer Befragung von Hausärzten auf, dass über die Hälfte die

\footnotetext{
34 Vgl. Zitate (P1:008), S. 62, (P30:381), S. 63 und (P2:055), S. 63

35 Zitat (P3:191-192), S. 64, vgl. auch (P20:338-342), S. 74
} 
Eigenschaften „merkwürdig“, „unattraktiv“, „,hässlich“ und „uncompliant“ mit übergewichtigen Menschen assoziierten. Die Sichtweise auf Übergewicht als Verhaltensproblem entspreche den negativen Stereotypen der übrigen Gesellschaft. Schorb (2009) schildert folgenden gesellschaftlich wahrgenommenen Zusammenhang von äußeren und ,inneren“ Eigenschaften:

„Der athletische Körper steht in der Dienstleistungsgesellschaft für Triebverzicht, Durchsetzungsstärke und Durchhaltevermögen. Der übergewichtige Körper wird dagegen zum Zeichen mangelnder Einsatzbereitschaft und fehlender Selbstdisziplin." (S.106)

Bezüglich der sozialen Bewertung von Übergewicht und Krankheit ist eine weitere Studie, auch wenn es dort nicht primär um Diabetes geht, erhellend. Daneski et al. (2010) untersuchten den historischen Hintergrund des gesellschaftlichen Diskurses bezüglich der Ursachen und der Behandlung von Schlaganfällen. Sie zeigten auf, dass die Empfehlung zur Lebensstilveränderung hinsichtlich Gewichtsabnahme und Bewegung in aktuellen britischen Leitlinien zur Prävention von Schlaganfällen in der Tradition medizinischer Lehrbücher des 18. Jahrhunderts steht. Über die letzten drei Jahrhunderte hinweg, so argumentieren Daneski et al., habe sich an den ärztlichen Empfehlungen zur Verhinderung eines Wiederauftretens oder eines erstmaligen Schlaganfalls kaum etwas geändert. Während ärztlicher Rat im 18. und 19. Jahrhundert durch die fälschliche Vorstellung von der Krankheit vorrausgehender „Fresssucht“ (gluttony) geprägt war, die moralisch verurteilt wurde, werde heute weiterhin empfohlen, abzunehmen, da Übergewicht mit Schlaganfällen assoziiert würde. Im 18. und 19. Jahrhundert wurde in medizinischen Schriften Korpulenz als „sichtbarer" Prädispositionsfaktor hervorgehoben und als Mangel an Moral verstanden. Jemand mit Übergewicht galt als schuldig für den eigenen Schlaganfall. Es gäbe immer noch ungenügend Evidenz dafür, dass Schlaganfällen direkt mit Übergewicht zusammenhängen. Dennoch - so Daneski et al. - finde sich diese Verbindung und die daraus resultierende Empfehlung den Lebensstil zu ändern, weiterhin auch in Lehrbüchern dieses Jahrhunderts. Zwar würde in einigen der Hinweis gegeben, dass diese Assoziation nicht in Randomized Controlled Trials (RCTs) bestätigt sei; dann würden sich Autoren jedoch auf einen Konsens der erfahrenden ärztlichen Berufsgruppe beziehen und die Lifestyle-Änderung trotzdem empfehlen.

Der Diskurs um Übergewicht habe sich ein wenig verändert. Heute werde nicht mehr das Individuum allein für seine eigene Lage für schuldig befunden, sondern zusätzlich 
werde Übergewicht als Indikator für moralischen Verfall einer ganzen Gesellschaft verstanden. Medien würden in dieser Hinsicht eine richtiggehende „moralische Panik“ verbreiten. Die Autoren zitieren Turner (2008), der den Diskurs so charakterisierte: das sich moralisch deviant verhaltende Individuum werde durch selbstverursachte Krankheit zu einer Last für den Staat. Daneski et al. argumentieren, dass die Angst vor moralischem Verfall durch Völlerei im 18. und 19. Jhd. sich heute in dem vermeintlichen Mangel an Willen zur Gesundheit wiederfinde. Der Fokus der Schlaganfallprävention bleibe erstaunlich konstant auf Essen, Bewegung und Gewicht gerichtet mit einer gleichermaßen konstanten moralischen Dimension auch in der heutigen säkularen Gesellschaft.

Vor dem Hintergrund der Moralisierung von Lebensstil ist es nicht erstaunlich, dass viele Interviewte „zugeben“, durch ihr Verhalten schuld an ihrer Krankheit zu sein. Aber nicht alle Interviewten, die sich selbst die Schuld an ihrer Erkrankung zusprechen, glauben auch, die Kondition selbst ändern zu können. ${ }^{36}$ Die beispielhaft genannten Kränkungen, wenn von Laborwerten und Körpermaßen auf das Verhalten und damit auf die Verantwortlichkeit geschlossen wird, sind viel eher ein Hinweis darauf, dass diese Verknüpfung in vielen Fällen demotiviert. Aufschlussreich für den Zusammenhang von Eigenverantwortung und Motivation ist die Art, wie Interviewte mit ihrer AuBenwirkung nach starker Gewichtsabnahme, also großer Veränderung der Körpermaße als sichtbarem Kriterium für Verzicht umgehen. Einer interviewten Frau war z.B. das Lob einer Bekannten für ihr neues Aussehen unangenehm, weil dies die Aufmerksamkeit auf die Gründe für ihre Diät lenkte. Abnehmen wegen Diabetes wird von ihr wie ein beschämender Beweis früherer „krankmachender“ Maßlosigkeit empfunden. ${ }^{37}$ Andere Interviewte haben dagegen nicht das Bedürfnis, ihre früheren Körpermaße oder ihr Verhalten zu verteidigen, möglicherweise, weil sie sich davon zum Zeitpunkt des Interviews wie von einer „überwundenen“ Lebensphase abgrenzen. ${ }^{38}$

Schamgefühle beschrieben auch Browne et al. (2013), die mithilfe von Interviews stigmatisierende Erfahrungen untersuchten, die australische Erwachsene mit Diabetes Typ 2 machten. Quellen von Stigmatisierung waren für sie die mediale Darstellung der

\footnotetext{
36 Vgl. Zitate (P16:0201-0203), S. 85 und (P27:110), S. 72

37 Vgl. Zitat (P20:91-95), S. 75

38 Vgl. Zitat (P32:258), S. 76
} 
Krankheit, aber auch Menschen im privaten Umfeld sowie medizinisches Personal. Im Kontakt mit medizinischem Personal erlebten die meisten Interviewten eine Mischung aus hilfreichen und entmutigenden Interaktionen. Einige berichteten von stigmatisierenden Erlebnissen, in denen der medizinische Fokus vor allem auf das gerichtet wurde, was „falsch“ war. Als Beispiele werden das Versagen beim Versuch der Gewichtsreduktion und der Senkung des HbA1c genannt. Solche Begebenheiten wurden als verurteilend und entmutigend erlebt. Eine Frau wird zitiert, die - P30 ähnlich - erzählt, dass sie zweimal in der Woche in einem Fitnessstudio Sport treibt. In der Diätberatung wird ihr aber bedeutet, dies sei eben nicht genug. Diese negativen Erlebnisse führten zu Arztwechseln, Vermeidung von medizinischen Kontrollterminen und der Suche nach anderen Beratungsquellen im Kreise von Familie und Freunden. Menschen aus dem privaten Umfeld wurden im Allgemeinen als Unterstützung erlebt, obwohl jede interviewte Person ein Negativbeispiel erzählte, z.B. wenn Menschen sich in ihre Ernährung einmischten. Eine Person beschreibt, wie sie darauf reagiert und den anderen zu verstehen geben will, dass sie sich ausgeschlossen fühlt, wenn sie nicht selbst entscheiden dürfe, was sie esse. Stigma erzeugte in vielen Interviewten psychischen Stress, führte zu Gefühlen wie Scham, Schuld, Bedauern, Hoffnungslosigkeit, niedrigem Selbstbewusstsein und Selbstwertgefühl. Auch Menschen, die nicht sichtbar übergewichtig waren oder eine ausgeprägte Familienanamnese aufwiesen, schienen das starke Gefühl zu haben, dass sie etwas hätten tun sollen, um die Krankheit zu vermeiden. Einige hatten den Eindruck, dass Diabetes Typ 2 ihren persönlichen Charakter in ein negatives Licht rückte. Zitiert wird eine Person, die die Krankheit die „blame and shame disease“ nennt.

In der Studie von Webb (2009) geht es darum, wie Adipositas von ärztlichem Personal und Adipösen gemeinsam zu einem moralischen Thema konstruiert wird. Die moralischen Dimensionen werden „sichtbar“, wenn Menschen auf die ärztlichen Eingangsfragen einer Konsultation im Rahmen eines Programms zur Gewichtreduktion in ihren Antworten klar zum Ausdruck bringen, ob sie „Erfolg“ hatten oder nicht. Auffällig ist, dass Menschen, die Gewicht verlieren, dies als ihren eigenen Verdienst darstellen, während andere, die auf die Eingangsfrage über „Misserfolg“ berichten, äußere, von innen nicht beeinflussbare Faktoren hervorheben. Webb argumentiert, dass bei der Behandlung von Übergewicht Diäten und Sport Priorität haben, diese Handlungen 
aber fernab des medizinischen Blicks geschehen. Das Sprechen oder Berichten über diese Handlungen ist daher Medium der Behandlung und selbst Behandlung (S. 856). Daher ist aus ihrer Sicht die Betrachtung von Arzt-Patient-Interaktionen so wichtig für die Forschung zu Adipositas. Dies trifft - nach unseren Ergebnissen - auch für die Diabetestherapie zu.

Die Menschen in Webbs Studie betonen ihre Bemühungen, sich von dem normativ abgewerteten Körper hin zu einem dünnen Körper zu bewegen. Sie stellen sich als gute Patienten dar, indem sie signalisieren, dass sie auf die ärztliche Meinung hören und willig sind, die Krankenrolle zu verlassen, und dafür möglichst großen Einsatz zeigen. Diese Beobachtungen vergleicht Webb mit Parsons „sick role“ (Parsons 1975), in der es Kranken temporär erlaubt ist, von den Normen abzuweichen, da sie nicht für ihre Krankheit verantwortlich gemacht werden. Gleichzeitig ist es jedoch ihre Pflicht, genesen zu wollen und die Krankenrolle zu verlassen. Diese Verantwortlichkeit der kranken Person, vermutet Webb, spielt bei Adipositas eine besondere Rolle, da sie als Lifestyle-Krankheit gilt und die Kranken das Privileg, zunächst nicht verantwortlich für ihre Kondition gemacht zu werden, oft nicht mal temporär genießen können. Daher ist der Druck höher, die „sick role“ verlassen zu wollen. Webb argumentiert, dass normativer Druck nicht automatisch in menschlichen Begegnungen existiert, sondern von den interagierenden Personen produziert wird. Diese Ergebnisse können möglicherweise eine Erklärung für das komplexe Erleben der Interviewten unserer Studie bieten, die in Interaktionen manchmal bereits Blicke des Gegenübers als Ausdruck moralischer Verurteilung empfanden.

Zusammenfassend lässt sich sagen, dass Interaktionen mit medizinischem Personal, aber auch dem alltäglichen sozialen Umfeld von der Angst geprägt sein können, anhand von Körpermaßen und Laborwerten als maßlos bewertet, moralisch verurteilt und persönlich abgelehnt zu werden. Die Aussagen der Interviewten zum Thema Körpermaße und die darin enthaltenen vielfältigen Versuche, die eigene moralische Identität zu schützen, spiegeln folgende gesellschaftlich verbreitete Vorstellungen wider:

1. Diabetes Typ 2 ist durch Übergewicht verursacht.

2. Übergewicht ist Resultat eines falschen, nämlich maßlosen Verhaltens.

3. Maßloses Verhalten reflektiert einen maßlosen, also schlechten Charakter. 
Angesichts dieses sozial akzeptierten Konstrukts - mit der Folge der Ablehnung „maßloser Menschen" - ist der große Aufwand, sich von den Dicken oder Maßlosen abzugrenzen bzw. zu erklären, dass das eigene Übergewicht/die Diabeteserkrankung noch andere Ursachen hat, nur zu verständlich.

\subsection{Eigene Regeln - Last oder Befreiung?}

„Verzicht wird geübt“ wurde als Überschrift für die dritte Subkategorie gewählt. In ihr sind Erfahrungen mit den alltäglichen Versuchen zu verzichten und Handlungsstrategien der Interviewten zusammenfasst. In dem Wort „üben“ klingt an, dass die Art und Weise, wie verzichtet wird, weder selbstverständlich noch einfach und beständig ist. Dies passt zu der Annahme von dynamischen Krankheitskonzepten, in denen ständig neue Strategien eingesetzt und Konsequenzen bewertet werden (Leventhal 1971; vgl. Kap. 2.5). Dass Menschen mit Diabetes Entscheidungen über ihr Gesundheitsverhalten nicht nur einmal treffen, sondern sich immer wieder, manchmal sogar täglich an die jeweilige Situation adaptieren müssen, ist anhand der Interviews nachvollziehbar (vgl. auch Paterson et al. 2001; vgl. Kap. 2.4).

Verzicht wird im Alltag geübt, weit entfernt von der ärztlichen Praxis. Vor dem Hintergrund der soeben diskutierten moralischen Bewertung von Verzicht bei gleichzeitig fehlenden äußeren Grenzen durch körperliche Einschränkungen, die zum Verzicht zwingen würden, möchten die Interviewten verständlicherweise Verantwortung für ihre Lebensgewohnheiten abgeben. Einige drücken dies in dem Wunsch nach dauerhafter Kontrolle von außen aus, z.B. durch eine bariatrische Operation oder - utopisch - durch das Festhalten auf einer „einsame[n] Insel“39. In manchen Fällen wirkt eine temporäre äußere Kontrolle erleichternd, z.B. durch im Krankenhaus verordnete Diät. ${ }^{40}$ Diese Ergebnisse erinnern an die Studie von Parry et al. (2005; vgl. Kap. 2.6), in der eine Interviewte Verantwortung dadurch abgeben wollte, dass das medizinische Personal ihr zu Hause den Kühlschrank mit den Lebensmitteln füllt, die sie essen dürfe. Parry et al. (2005) weisen darauf hin, dass dieser Wunsch der aktuellen Forderung nach aktivem Selbstmanagement widerspricht.

${ }^{39} \mathrm{Vgl}$. Zitat (P27:102), S. 80

40 Vgl. Zitate (P8:0600-0606), S. 81 und (P16:0199), S. 81 
Ungeachtet der genannten Möglichkeiten, Verantwortung temporär abzugeben, müssen die Interviewten aber oft selbst entscheiden, ob und wie sie verzichten. Dabei brauchen sie Konzepte, an denen sie sich bei Essensauswahl, Zubereitung etc. orientieren. In der Analyse konnten fallübergreifend ähnliche Merkmale dieser Konzepte identifiziert werden. Diese Konzepte habe ich „Regeln“ genannt. Regeln sind Konzepte darüber, was richtig und falsch ist. Schwierig wird verzichten dadurch, dass zwar die Ziele definiert sind, die Art und Weise wie verzichtet werden soll, aber weniger klar ist. In den Nationalen Versorgungsleitlinien zur Therapie des Typ-2-Diabetes werden praktische Empfehlungen angeboten, wie z.B. „kein generelles Zuckerverbot, jedoch Vermeiden von großen Mengen an Haushaltszucker [...]“, ,große Portionen und häufigen Verzehr von fetten Lebensmitteln, z.B. fettes Fleisch, fette Wurstwaren, fetter Käse, fette Backwaren, fette Fertigprodukte, fettes Fast-Food, Sahne, Schokolade, Chips usw. vermeiden“. Daneben heißt es, Menschen mit Diabetes solle geraten werden, „die Vielfalt des Lebensmittelangebotes [zu] nutzen und [zu] genießen“ (Bundesärztekammer (BÄK), Kassenärztliche Bundesvereinigung (KBV), Arbeitsgemeinschaft der Wis-senschaftlichen Medizinischen Fachgesellschaften (AWMF) 2013). Nicht nur wird beklagt, dass die Ernährungsforschung bei Diabetes Typ 2 wissenschaftlich ungenügend ist (Nield et al. 2008); die Umsetzung solcher Diätempfehlungen ist ein Problem (Peyrot et al. 2005). Gauthier-Chelle et al. (2004) verglichen die Ernährungsgewohnheiten von Menschen mit und ohne Diabetes in Frankreich und kamen zu dem Schluss, dass Menschen mit Diabetes durchaus versuchten, ihre Ernährung zu verbessern und ihre Ernährung umstellten, häufig aber nicht auf die richtige Weise, z.B. sei der Nahrungsanteil an Proteinen zu hoch. Sie vermuten, dass Menschen mit Diabetes Typ 2 verwirrende und widersprüchliche Ratschläge von zahlreichen Seiten, nämlich nicht nur medizinischem Personal, sondern auch aus den Medien und von Bekannten erhalten würden.

Ingadottir und Halldorsdottir (2008) beschreiben, dass die von innen zum subjektiven Erleben von Diabetes ${ }^{41}$ befragten Personen den starken Wunsch hatten, "richtig“ $z u$ handeln, ein für die Autorinnen wichtiges Motiv menschlichen Handelns. Was aber als „richtig“ bewertet wird, sei nicht immer klar, zumal sich in der Diabetesversorgung viel

${ }^{41}$ Alle bis auf eine Person hatten Diabetes Typ 1. Zum Studiendesign siehe Kap. 2.7. 
verändert habe. Die Strenge des Behandlungsplans würde sich von einem Arzt zum nächsten unterscheiden. Dennoch wüssten Menschen, wenn sie etwas richtig und falsch machten, wenn sie Grenzen überschritten, die sie sich selbst gesetzt hatten. Diese Hypothese passt zu den Ergebnissen der hier vorliegenden Studie: So schmerzhaft es für viele Interviewten war, mit der Aufgabe zu verzichten allein gelassen zu werden (vgl. Kap. 5.4.4), so hatten sie doch zum Teil sehr genaue Vorstellungen über "gute“ und „schlechte“ Lebensmittel (vgl. Kap. 5.6.3). Die Interviewten kategorisierten Speisen und Verhaltensweisen in „gesund“ und „ungesund“. Genau wie bei der äußeren und inneren Bewertung von Verzicht war diese Kategorisierung von einem Druck geprägt, sich von Maßlosigkeit abzugrenzen und „richtig“ zu verzichten.

Selbstverständlich ist es von Bedeutung, ob die Interviewten den Eindruck haben, Laborwerte und Gewicht, also medizinische, äußere Erfolgskriterien durch ihr Verhalten unmittelbar beeinflussen zu können. Können sie mit einer gewissen Verlässlichkeit die Werte als Konsequenz eines bestimmten Essverhaltens voraussagen, ist es möglich, Lebensmittel zu bestimmen, die sie nicht essen dürfen. Damit entsteht zugleich ein Handlungsspielraum, weil sich Lebensmittel identifizieren lassen, die die Werte wenig oder gar nicht verändern. Sogar „Windbeutel“ scheinen dann „medizinisch gesehen“, erlaubt, wenn die Werte zeigen, „dass das nicht so falsch ist“, sie zu essen. ${ }^{42}$ Verzicht wird also z.T. in einer Weise ausgelegt, dass es nicht mehr Verbot allen Genusses ist und so an negativer Bedeutung verliert. Zum Teil kann durch verbesserte Laborwerte oder Gewichtsabnahme - fast wie durch eine Belohnung - der Ehrgeiz entstehen, zu verzichten. ${ }^{43}$

Werden die Laborwerte und Gewicht dagegen nicht als beeinflussbar erlebt ${ }^{44}$, ist es nur logisch, dass sie keine praktische Orientierung bieten. Abwendung von diesen Erfolgskriterien als alltäglichem Maßstab ist die Folge. Eine Interviewte übernahm Konzepte, die in ihrer Selbsthilfegruppe als „Tipps“ angeboten wurden, auch wenn sie den Eindruck hatte, dass sich ihre Laborwerte daraufhin verschlechterten. ${ }^{45}$ Als Orientierung dient in so einem Fall dann eher das eigene Wohlbefinden und die Überzeugung,

\footnotetext{
42 Vgl. Zitat (P21:534), S. 86

43 Vgl. Zitat (P16:1474-1483), S. 85

44 Vgl. Zitat (P30:093), S. 86

45 Vgl. Zitat (P30:302), S. 87
} 
richtig zu handeln, in diesem Fall bestätigt durch die Selbsthilfegruppenleiterin, der Expertise zugesprochen wird.

Diese Beobachtungen sind vergleichbar mit den Ergebnissen der qualitativen Studie von Peel et al. (2004) zu Erfahrungen mit selbstständiger Blutzuckermessung von Menschen mit Diabetes Typ 2 in Schottland. Vor dem Hintergrund, dass der medizinische Nutzen der Blutzuckerselbstmessung noch immer umstritten sei, diese aber als Chance gelte, größere Partnerschaft in der Arzt-Patient-Beziehung zu fördern und immer mehr Menschen mit entsprechenden Geräten versorgt würden, stellen Peel et al. (2004) die psychologischen Auswirkungen zur Diskussion. Sie fanden heraus, dass niedrige Blutzuckerwerte die Interviewten mit Stolz erfüllten und überzeugten, sich richtig verhalten zu haben. Über negative Erfahrungen berichteten meist Interviewte mit ungünstigen Blutzuckerwerten. Nicht durch das Verhalten erklärbare zu hohe Blutzuckerwerte führten zu Stress, dem Gefühl, persönliche Schuld zu tragen, und manchmal zur Abwendung von den therapeutischen Empfehlungen. Die Selbstmessung intensivierte die Vorstellung von „Erfolg“ oder „Versagen“ des Selbstmanagements (Peel et al. 2004). Dies unterstützt die Ergebnisse der vorliegenden Studie, dass die Bewertung der „sichtbaren“ Erfolgskriterien als Bewertung der eigenen Person empfunden wird. Dementsprechend kann eine Orientierung an den „Erfolgen“ guter Laborwerte möglicherweise nur Menschen helfen, „Verzichts-Regeln“ abzuleiten, die den Eindruck haben, die Werte selbst beeinflussen zu können, und tatsächlich „Erfolgserlebnisse“ haben.

Es geht in der hier vorliegenden Studie gerade nicht um Erfolg oder Misserfolg - und damit um die Kategorisierung von Menschen, denen die Lebensstilumstellung gelungen ist, und jenen, denen sie nicht gelungen ist. Es konnte aber beobachtet werden, dass Motivation und die Vorstellung, Einfluss auf die Gesundheit nehmen zu können oder nicht, eine große Rolle in der Krankheitserfahrung spielt. Viele Interviewte suchen „Erfolgserlebnisse“. „Regeln“, die zu den eigenen Bedürfnissen passen, können dabei helfen. Ich möchte beispielhaft eine Interviewte erwähnen, die das eigene Verhalten positiv bewertet z.B., wenn sie statt Butter Senf als Brotbelag verwendet („Man muss [...] für sich selber so ein bisschen was finden, [...] wo man so sagt: "Guck mal, da habe ich mir das gespart." [...] Wo man sich so was spart und, und trotzdem auch zufrieden bei ist.“ [P20:393]). Dieses Beispiel zeigt, wie es Interviewten gelingt, sich 
bei dem Erfüllen einer Regel - also zunächst ohne eine anschließende Bewertung des Verzichts anhand der „sichtbaren“ Erfolgskriterien - erleichtert zu fühlen. Es macht sie froh, verzichtet zu haben und eigenen moralischen Ansprüchen gerecht geworden zu sein. Auch Ingadottir und Halldorsdottir (2008) zeigen in ihrer Interview-Studie, dass "das Richtige tun" unmittelbar zu einem physischen und psychischen Wohlbefinden führt.

Am häufigsten sprachen Interviewte über Regeln, die bestimmten, welche Lebensmittel erlaubt waren und welche nicht. Dabei wurde eine gewisse Symbolik in den Annahmen über "gute" und "schlechte" Speisen deutlich. Viele emotional bedeutsame Speisen schienen verboten zu sein, z.B. Eis, Kuchen, Braten etc. In einigen Interviews wurden Annahmen über geschlechtstypische Lebensmittelvorlieben bzw. -bedürfnisse deutlich, z.B. dass Männer gerne viel Fleisch essen und wenig Gemüse (P21), was zu den Ergebnissen der Arbeit von Broom und Lenagh-Maguire (2010) passt. Auch generationstypische Vorstellungen über Essen spielten eine Rolle, wie z.B. die Erfahrung von Mangelerscheinungen bei Verwandten in der Nachkriegszeit. Wurst, Kartoffelpuffer - diese Speisen wurden mit Attributen wie stark und nahrhaft verknüpft.

Gesundes wurde oft eher abfällig erwähnt ${ }^{46}$ : als „trocken“, „langweilig“, wenig nahrhaft - besonders, wenn Interviewte sich mit Mitmenschen verglichen, die vermeintlich völlig unbeschwert Speisen auswählten. Manche Interviewte stellten aber auch Vergleiche in eine andere Richtung an: Eine Interviewte spricht davon, nie der „Tortentyp“ gewesen zu sein, d.h., sprach eher abfällig über solchen Genuss. Durch diesen Abgrenzungsmechanismus gelingt es ihr sogar, andere Süßspeisen als etwas Gesünderes darzustellen: „Torte nicht zu essen fällt mir sowieso nicht schwer. War nie mein Ding. Aber so schön Kuchen [...] wenn ich denn noch mal rüber gehe nachmittags zum Bäcker und da, der hat dann [...] frische Windbeutel“ (P21:534). Die gleiche Interviewte vergleicht sich mit Menschen, die ganze Vollmilchschokoladentafeln essen, während sie sich "nur“ dunkle Schokolade und Rotwein erlaubt, und definiert diese Lebensmittel auf diese Weise als gesund. Eine Interviewte hob ihre Vorliebe für Obst als Teil eines gesunden Lebensstils hervor, während andere Interviewte darüber klagten, dass sogar

46 „....immer Karotte oder eine Tomate essen: Wer kann das schon?“ (P30:272) 
Fruchtsüße nicht erlaubt sei. Was an diesen Beispielen deutlich werden soll: Angesichts der medialen Vielfalt an Ratschlägen ist es eigentlich ein Leichtes, alle möglichen Speisen (in Maßen) als gesund zu definieren. Eigene Regeln können dadurch manchmal befreiend sein: Es ist eben nicht eindeutig definiert, was "richtig“ und „falsch" ist.

Eine Besonderheit stellten Lebensmittel dar, die eigentlich „verboten“ waren, aber die Interviewte sich dennoch erlaubten: wenn z.B. Personen merkten, dass ihre Laborwerte sich trotz gelegentlichen Genusses dieser Speisen nicht veränderten oder wenn sie sich in der Lage fühlten, die Blutzuckerwerte danach durch das Einhalten eines noch strikteren Diätplans wieder zu regulieren. Außerdem waren Dinge erlaubt, die einen „wirklichen“, also verdienten Genuss darstellten oder einen „Jieper" befriedigten und damit für Wohlbefinden und für den weiteren Erfolg des Verzichts von Bedeutung waren. Dabei war der Druck, diese Übertretung von Regeln vor sich selbst und anderen zu rechtfertigen, aber weiterhin oft so groß, dass Interviewte dann z.B. betonten, nur "ein Löffelchen Mayonnaise" zu sich zu nehmen. Ingadottir und Halldorsdottir (2008) beschrieben ganz ähnlich, dass Verbotenes manchmal im Sinne der Lebensqualität akzeptiert werde.

Obwohl Verzicht generell eher mit einer schmerzhaften Aufgabe von alten Gewohnheiten und Ausgeschlossenheit assoziiert wurde, erzählten einige Interviewte von neuen Gewohnheiten, in denen sie sich bewusst von alltäglichen Aktivitäten der Mitmenschen ausschlossen. Als Beispiel möchte ich einen Interviewpartner nennen, der eine Stunde Fahrradfahren nach der Arbeit als private „Auszeit“ entdeckte, in der er nicht an Arbeit oder Familie denken musste. Eine Interviewte entwickelte den Vorsatz, sich täglich einige Minuten Pause vom Familienleben und Zeit für sich zu nehmen. Diese Abgrenzungen wirken wie Befreiungen. Ein Sonderfall stellt sicherlich ein Interviewpartner dar, welcher statt des Gefühls, durch Verzicht ausgeschlossen zu werden und sich abgrenzen zu müssen, eine intensive Verzichtsgemeinschaft während des Ramadan erlebt. Mehrere Interviewten erzählten, wie sie sich durch Sonderbehandlungen nicht aus dem Familienkreis aus-, sondern eingeschlossen fühlten, z.B. wenn Familienmitglieder spezielles Essen für sie vorbereiteten, „für Diabetiker geeignete“ Süßigkeiten kauften oder sie bei körperlicher Aktivität begleiteten. 
Yamakawa and Makimoto (2008) beschäftigten sich mit positiven Erfahrungen bei Diabetes Typ 2. Sie stellten fest, dass viele der in Japan interviewten Menschen die Diagnose als Gelegenheit betrachteten, ihr Leben zu verändern. Außerdem gaben viele an, dass sich familiäre Beziehungen verbessert hätten. Aktuelle Ergebnisse einer Studie zu sportlicher Aktivität von postmenopausalen Frauen legen nahe, dass die Diagnose Diabetes zu Erhöhung der Aktivität motivieren können (Schneider et al. 2013).

Regeln können also rigide und flexibel sein, eine radikale Veränderung oder sanfte Anpassung an den früheren Alltag darstellen, Ausschluss oder Integration in die soziale Gemeinschaft bedeuten. Positiv besetzt scheinen Regeln zu sein, die zu bewältigen sind, weil sie zu den eigenen Bedürfnissen passen.

\subsection{Praktische Implikationen}

„Verzicht" beherrscht die Krankheitserfahrungen der Interviewten fallübergreifend und führt vorübergehend oder beständig zum subjektiven Eindruck, auf ein genussvolles Leben verzichten zu müssen. Dies finden die Interviewten auf der einen Seite ungerecht, insbesondere weil andere Menschen scheinbar nicht verzichten müssen. Auf der anderen Seite stehen sie oder fühlen sie sich aber unter einem stark verinnerlichten moralischen Druck. Körpermaße und Laborwerte werden zu „sichtbaren“ Prüfmarken eines gelungenen Verzichts oder eines maßlosen Lebensstils. Das „Verzichtenmüssen" prägt zugleich die Arzt-Patient-Beziehung. Im Sprechzimmer - so die Erfahrung oder Wahrnehmung - wird der Erfolg der Lebensstiländerung immer wieder beurteilt. Aber auch im Privatleben erfahren die Interviewten, wie von ihren Körpermaßen auf ihr Verhalten und auf ihren Charakter geschlossen wird. Die daraus folgende Einteilung in genügsame (schlanke) und maßlose (dicke) Menschen hat sich allen Interviewten eingeprägt. Alle bemühen sich dementsprechend um eine Abgrenzung von Maßlosigkeit und maßlosen Menschen.

Auch wenn aus der Beschäftigung mit Krankheitskonzepten zunächst keine konkreten und allgemein gültigen Handlungsvorschläge abzuleiten sind bzw. Zurückhaltung vor übereilten Schlussfolgerungen geboten ist, haben die Ergebnisse auch praktische Implikationen. Das Bewusstsein für die Eigenverantwortung und die Forderung nach aktiver Selbstbehandlung haben Menschen mit Diabetes Typ 2 offensichtlich verinnerlicht. Gleichzeitig erleben sie alltäglich eine moralische Bewertung ihres Lebensstils 
durch ärztliches Personal: eine stets drohende Diskreditierung bei „schlechten“ Laborwerten und Übergewicht. Dadurch entsteht eine stark empfundene Abhängigkeit vom ärztlichen Urteil, die einer angstfreien lösungsorientierten Zusammenarbeit oft im Wege steht. Eine kollaborative Arzt-Patient-Beziehung, wie sie zumindest auf Forschungsebene schon lange angestrebt wird (z.B. Anderson und Funnell 2000, Paterson 2001), kann so in vielen Fällen nicht erreicht werden.

Aus ärztlicher Sicht stellt sich die Frage, wie Kommunikation über den Lebensstil wertfreier - und damit angstfreier - gestaltet werden kann. Die Nichtbeachtung von „unsichtbaren" Anstrengungen bei gleichzeitiger übermächtiger Bedeutung von Körpermaßen und Laborwerten schmerzte die Betroffenen besonders. Einige der Interviewten deuteten die Hoffnung an, dass Ärztinnen und Ärzte ihre Verzichts-Bemühungen in ihrem persönlichen Lebenskontext anerkennen würden. Sie wünschten sich ein empathisches, nicht aufgesetztes oder angelerntes Verständnis dafür, dass Verzicht schwierig ist. Dass die Auseinandersetzung mit Verzicht und Maßlosigkeit heutzutage nicht nur Menschen mit Diabetes Typ 2 und Übergewichtigen führen, sondern sie in der ganzen Gesellschaft verbreitet ist, könnte eine sensible Kommunikation über den Lebensstil erleichtern. Beide Seiten der Arzt-Patient-Beziehung sollten begreifen, dass die Laborwerte und Körpermaße nicht das Verhaltens und schon gar nicht Intention oder Willenlosigkeit unmittelbar abbilden (vgl. Parry et al. 2005). Statt die Betroffenen durch wohlgemeinte Vorschläge und psychologisierende Hilfestellungen noch weiter unter Druck zu setzen, sollte man sie besser auf eine moralische „Falle“ hinweisen. Bei beabsichtigter Lebensstilumstellung können sie in Gefahr sein, Laborwerte und Körpermaße zu „persönlich“ zu nehmen, sich selbst zu verurteilen und sich hilflos und allein gelassen zu fühlen.

Um (angehendem) medizinischen Personal die spezifischen Probleme von Menschen mit Diabetes Typ 2 bewusst zu machen, könnten Ergebnisse dieser Arbeit in Form von authentischen Fallbeispielen zu Lehrmaterial ausgearbeitet werden. So könnte die Perspektive der Betroffenen zum Gegenstand medizinischer Ausbildung werden (Kumagai et al. 2009; Law 2011; Field und Ziebland 2008). Dies ist auch insofern sinnvoll, als dass es viele weitere Krankheiten gibt, für die es sich lohnt, auf Kommunikation über Lebensgewohnheiten vorzubereitet zu sein, z.B. Bluthochdruck. Weiterführende 
Forschung könnte den Austausch von medizinischem Personal und Menschen mit Diabetes in Fokusgruppen zu den Themen Verzicht und Maßlosigkeit beinhalten, um kommunikative „Stolpersteine“ sichtbar werden zu lassen.

Doch nicht nur auf der Ebene der Arzt-Patient-Kommunikation sollte für den moralischen Druck sensibilisiert werden, der sich aus der Betonung von Eigenverantwortung ergibt. Bei der medialen Präsentation von Diabetes Typ 2 sollte zum Ausdruck gebracht werden, dass Lebensstilveränderungen Teil der Behandlungsempfehlungen sein können, die Ursachen für die Erkrankung und ihr Fortschreitens aber komplex sind und die Betroffenen nicht oder nicht unmittelbar intentional „schuld“ an der Erkrankung sind.

Mit diesen hier zur Diskussion gestellten Empfehlungen soll nicht der Zusammenhang von Diabetes und Verhalten bestritten werden. Natürlich kann es für das Individuum wertvoll sein, sich zu fragen, ob eine Umstellung des Lebensstils das Risiko verringert, an Diabetes zu erkranken oder Komplikationen zu vermeiden. Hier Handlungsspielräume zu eröffnen, ist allemal sinnvoll. Ein gesunder Lebensstil sollte dennoch nicht zur allgemeinen Pflicht erhoben werden und scheinbar uneinsichtige Kranke nicht gleich als "Gesundheitsverweigerer" charakterisiert werden. Solange der Zusammenhang von Lebensstil, Gewicht und Diabetes nicht präzise geklärt ist, empfiehlt sich Zurückhaltung gegenüber gradlinigen Verhaltensratschlägen (Franks 2012; Nolan et al. 2011), um Stigmatisierung entgegenzuwirken (Teixeira und Budd 2010; Browne et al. 2013).

Der Druck zur Lebensumstellung erzeugt im Alltag die Notwendigkeit, eigene „Verzichts-Regeln" zu formulieren. Der Dualismus von Verzicht und Maßlosigkeit dringt dabei bis in kleinste - banal erscheinende - Alltagsentscheidungen vor, z.B. die Einteilung in „gesunde“ und „ungesunde“ Lebensmittel. Dies ist ernährungsphysiologisch manchmal fraglich. Bevor man aber über die Laien-Logik lächelt, ist das Motiv und Handlungspotential solcher Strategien zu sehen: Die Interviewten erkämpfen sich eine gewisse Freiheit, Regeln nach ihren Bedürfnissen zu gestalten, z.B. bestimmte Lebensmittel im Vergleich mit anderen als "gesund“ oder "gesünder" zu bewerten. Bei der Gestaltung der Regeln orientieren sie sich an den „sichtbaren“ Erfolgskriterien, beziehen aber auch „unsichtbare“ mit ein: die Erfüllbarkeit der Regeln und die Steigerung 
des eigenen Wohlbefindens. Verzicht kann dann manchmal zu einer positiven, „befreienden" Abgrenzung von üblichen Lebensgewohnheiten führen.

Damit endet die Arbeit mit einem positiven Ausblick. Das Problem in der Diabetesbehandlung ist nicht das geringe Bewusstsein für die Notwendigkeit einer Lebensstilveränderung. Möglicherweise kann es hilfreich sein, Menschen mit Diabetes Typ 2 zuzuhören, welche „Regeln“ sie aufgestellt haben, und innen Raum für die „unsichtbaren“ Bemühungen zu geben. 


\section{Zusammenfassung}

Hintergrund: Bei Diabetes Typ 2 gilt die Selbstbehandlung als wesentlicher Therapiebestandteil. In vielen Forschungsarbeiten geht es deshalb um Ansatzpunkte, wie man Erkrankte besser zu gesundheitsfördernden Maßnahmen motivieren kann. Solche gutgemeinten - pädagogischen Zielsetzungen verstellen oft den Blick auf das subjektive Erleben dieser Menschen. Fragestellung: In der vorliegenden Arbeit standen daher die Krankheitserfahrungen bei Diabetes Typ 2 im Vordergrund. Während des Analyseprozesses stellte sich „Verzicht“ als beherrschende Erfahrung heraus. Daher sollte die Ausstrahlung der Verzichtserfahrung in alle Lebensbereiche, vor allem als mögliche Belastung für die Krankheitsbewältigung, die sozialen Interaktionen und für die alltägliche Handlungsorientierung untersucht werden. Methode: Als Material lagen 35 für das Website-Projekts "krankheitserfahrungen.de" erhobene narrative Interviews vor. Aus diesen wurden im Sinne eines Theoretical Samplings Ausschnitte aus Interviews mit 14 Personen ausgewählt und nach Methoden der Grounded Theory ausgewertet. Ergebnisse: Alle Interviewten standen unter dem Druck, auf ein genussvolles Leben zu verzichten, was innen ungerecht erschien. Als „Prüfmarken“ gelungenen Verzichts wurden Laborwerte und Körpermaße herangezogen - von Ärzten, aber auch von Personen im privaten sozialen Umfeld und von den Interviewten selbst. Diese „sichtbaren“ Kriterien wurden manchmal positiv im Sinne eines selbst erreichten Ergebnisses („Verzicht“) erlebt, manchmal abgewehrt als ungerechte äußere Beurteilung ihres Verhaltens (als "Maßlosigkeit"). Das Denken in den Kategorien Verzicht und Maßlosigkeit ging mit der ständigen Angst vor moralischer Diskreditierung einher und fand auch Eingang in die eigenen „Regeln“, in denen Interviewte selbst bestimmten, was "gesunde" und „ungesunde" Lebensmittel sind. Dabei ergab sich eine gewisse Freiheit, diese Regeln als zu den Bedürfnissen passend zu gestalten. Manchmal konnte Verzicht so zu positiven neuen Erfahrungen führen. Schlussfolgerungen: Menschen mit Diabetes Typ 2 haben die medizinische und gesellschaftliche Aufforderung nach Verzicht verinnerlicht und stehen dadurch unter starkem Druck. Statt diesen durch pädagogische Strategien noch weiter zu erhöhen, erscheint es sinnvoller, wenn Ärzte mit ihren Patienten über deren „unsichtbare“ Bemühungen sprechen und sie dabei begleiten. 


\section{Literaturverzeichnis}

Agardh E, Allebeck P, Hallqvist J, Moradi T, Sidorchuk A (2011): Type 2 diabetes incidence and socio-economic position: a systematic review and meta-analysis. Int J Epidemiol $\underline{40}$, 804818

Ahlin K, Billhult A (2012): Lifestyle changes - a continuous, inner struggle for women with type 2 diabetes: a qualitative study. Scand J Prim Health Care $\underline{30}, 41-47$

Al-Sharafi BA, Gunaid AA (2014): Prevalence of Obesity in Patients With Type 2 Diabetes Mellitus in Yemen. Int J Endocrinol Metab 12, e13633; doi: 10.5812/ijem.13633

Anderson RM, Robins LS (1998): How do we know? Reflections on qualitative research in diabetes. Diabetes Care 21, 1387-1388

Anderson RM, Funnell MM (2000): Compliance and Adherence are Dysfunctional Concepts in Diabetes Care. Diabetes Educ 26, 597-604

Arbeitsgruppe krankheitserfahrungen.de (2013): krankheitserfahrungen.de: Erfahrungen mit Gesundheit, Krankheit und Medizin. Online unter: http://krankheitserfahrungen.de (aufgerufen am 23.09.2014)

Atlas.ti GmbH (Copyright 1993-2014): Atlas.ti Version 7

Bailey CJ, Kodack M (2011): Patient adherence to medication requirements for therapy of type 2 diabetes. Int J Clin Pract $\underline{65}$, 314-322

Bailey CJ, Aschner P, Del Prato S, LaSalle J, Ji L, Matthaei S (2013): Individualized glycaemic targets and pharmacotherapy in type 2 diabetes. Diab Vasc Dis Res $\underline{10}, 397-409$

Bandura A (1977): Self-efficacy: Toward a unifying theory of behavioral change. Psychol Rev 84, 191-215

Blumer H: Der methodologische Standort des symbolischen Interaktionismus; in: Alltagswissen, Interaktion und gesellschaftliche Wirklichkeit; hrsg. v. Arbeitsgruppe Bielefelder Soziologen; Rowohlt, Reinbek bei Hamburg 1981, 80-146

Braga MFB, Casanova A, Teoh H, Gerstein HC, Fitchett DH, Honos G, McFarlane PA, Ur E, Yale J, Langer A et al. (2012): Poor achievement of guidelines-recommended targets in type 2 diabetes: findings from a contemporary prospective cohort study. Int J Clin Pract $\underline{66}$, 457464

Breuer F: Reflexive Grounded-Theory: Eine Einführung für die Forschungspraxis. (Lehrbuch), 1. Auflage; VS Verlag für Sozialwissenschaften, Wiesbaden 2009

Bröckling U (2008): Vorbeugen ist besser ... Zur Soziologie der Prävention. Behemoth. A Journal on Civilisation 1, 38-48

Broom D, Treacher G (2003): Representing gender in publications for diabetics. Health Sociol Rev $\underline{12}, 55-67$

Broom D, Whittaker A (2004): Controlling diabetes, controlling diabetics: moral language in the management of diabetes type 2. Soc Sci Med $\underline{58}$, 2371-2382

Broom DH, Lenagh-Maguire A (2010): Gendered configurations of diabetes: from rules to exceptions. J Gend Stud 19, 195-209

Browne JL, Ventura A, Mosely K, Speight J (2013): 'I call it the blame and shame disease': a qualitative study about perceptions of social stigma surrounding type 2 diabetes. BMJ Open $\underline{3}$, e003384; doi: 10.1136/bmjopen-2013-003384

Brunetti A, Chiefari E, Foti D (2014): Recent advances in the molecular genetics of type 2 diabetes mellitus. World J Diabetes $\underline{5}, 128-140$ 
Bucher A: Geiz, Trägheit, Neid \& Co. in Therapie und Seelsorge: Psychologie der 7 Todsünden. Springer-Verlag, Berlin 2012

Bundesärztekammer (BÄK), Kassenärztliche Bundesvereinigung (KBV), Arbeitsgemeinschaft der Wissenschaftlichen Medizinischen Fachgesellschaften (AWMF) (2013): Nationale VersorgungsLeitlinie Therapie des Typ-2-Diabetes. Kurzfassung, Version 3.

Online unter: http://www.versorgungsleitlinien.de/themen/diabetes2/dm2_therapie (aufgerufen am 23.09.2014)

Burris S (2008): Stigma, ethics and policy: A commentary on Bayer's "Stigma and the ethics of public health: Not can we but should we". Soc Sci Med 67, 473-475

Butt G, Paterson BL, McGuinness LK (2007): Living With the Stigma of Hepatitis C. West J Nurs Res $\underline{30}, 204-221$

Centers for Disease Control and Prevention (2011): National diabetes fact sheet: national estimates and general information on diabetes and prediabetes in the United States, 2011. Online unter: http://www.cdc.gov/diabetes/pubs/factsheets.htm (aufgerufen am 23.09.2014)

Charmaz K: Constructing grounded theory: A practical guide through qualitative analysis; Sage Publications, London 2006

Chatterjee JS (2006): From compliance to concordance in diabetes. J Med Ethics $\underline{32}, 507-$ 510

Cohen MZ, Tripp-Reimer T, Smith C, Sorofman B, Lively S (1994): Explanatory models of diabetes: patient practitioner variation. Soc Sci Med $\underline{38}, 59-66$

Colagiuri S, Borch-Johnsen K, Glümer C, Vistisen D (2005): There really is an epidemic of type 2 diabetes. Diabetologia $\underline{48}, 1459-1463$

Collins MM, Bradley CP, O'Sullivan T, Perry IJ (2009): Self-care coping strategies in people with diabetes: a qualitative exploratory study. BMC Endocr Disord $\underline{9}$ :6; doi:10.1186/1472-68239-6

Corbin JM: Grounded Theory; in: Hauptbegriffe qualitativer Sozialforschung; hrsg. v. Bohnsack R, Marotzki W, Meuser M; (UTB 8226), 3. Auflage; Budrich, Opladen 2011, 70-75

Coyne IT (1997): Sampling in qualitative research. Purposeful and theoretical sampling; merging or clear boundaries? J Adv Nurs $\underline{26}$, 623-630

Daneski K, Higgs P, Morgan M (2010): From gluttony to obesity: moral discourses on apoplexy and stroke. Sociol Health IIIn $\underline{32}, 730-744$

Daousi C, Casson IF, Gill GV, MacFarlane IA, Wilding, J P H, Pinkney JH (2006): Prevalence of obesity in type 2 diabetes in secondary care: association with cardiovascular risk factors. Postgrad Med J $\underline{82}, 280-284$

Dieterich A (2007): The modern patient - Threat or promise? Physicians' perspectives on patients' changing attributes. Patient Educ Couns $\underline{67}, 279-285$

Duden: Das Bedeutungswörterbuch; hrg. v. d. Dudenredaktion [Red. Bearb.: Birgit Eickhoff]; Band 10. (Der Duden in zwölf Bänden), 3. Auflage; Dudenverlag, Mannheim 2002

Dunstan D, Zimmet P, Welborn T, Sicree R, Armstrong T, Atkins R, Cameron A, Shaw J, Chadban S: Diabesity and associated disorders in Australia - 2000. The accelerating epidemic. The Australian diabetes, obesity and lifestyle study (AusDiab). International Diabetes Institute, Melbourne 2001. Online unter: http://www.health.gov.au/internet/main/publishing.nsf/Content/pq-diabetes-pubs-diabesity (aufgerufen am 24.09.2014) 
Eriksson KF, Lindgärde F (1991): Prevention of Type 2 (non-insulin-dependent) diabetes mellitus by diet and physical exercise The 6-year Malmö feasibility study. Diabetologia $\underline{34}$, 891898

Field K, Ziebland S (2008): Beyond the Textbook: A preliminary survey of the uses made of the DIPEX website (www.dipex.org) in healthcare education. Online unter: http://healthtalkonline.org//learning-and-teaching/using-healthtalkonline (aufgerufen am 23.09.2014)

Fischer P: Phänomenologische Soziologie. Transcript-Verlag, Bielefeld 2012

Foster GD, Wadden TA, Makris AP, Davidson D, Sanderson RS, Allison DB, Kessler A (2003): Primary care physicians' attitudes about obesity and its treatment. Obes Res $\underline{11}, 1168-1177$

Franks PW (2012): The Complex Interplay of Genetic and Lifestyle Risk Factors in Type 2 Diabetes: An Overview. Scientifica (Cairo) 2012, Article ID 482186. doi: 10.6064/2012/482186

Gauthier-Chelle K, Mennen L, Arnault N, Rigalleau V, Hercberg S, Gin H (2004): Comparison of the diet of self-declared diabetics with non-diabetic patients in the SU.VI.MAX study: did the diabetics modify their nutritional behavior? Diabetes Metab $\underline{30}, 535-542$

Gillibrand W, Flynn M (2001): Forced externalization of control in people with diabetes: a qualitative exploratory study. J Adv Nurs $\underline{34}, 501-510$

Glaser BG, Strauss AL: The discovery of grounded theory: Strategies for qualitative research. (Observations), Aldine, New York 1967

Gollust SE, Lantz PM (2009): Communicating population health: Print news media coverage of type 2 diabetes. Soc Sci Med $\underline{69}, 1091-1098$

Guh DP, Zhang W, Bansback N, Amarsi Z, Birmingham CL, Anis AH (2009): The incidence of co-morbidities related to obesity and overweight: A systematic review and meta-analysis. BMC Public Health $\underline{9}, 88$

Harvey JN, Lawson VL (2009): The importance of health belief models in determining self-care behaviour in diabetes. Diabet Med 26, 5-13

Heidemann C, Du Y, Schubert I, Rathmann W, Scheidt-Nave C (2013): Prävalenz und zeitliche Entwicklung des bekannten Diabetes mellitus. Bundesgesundheitsblatt Gesundheitsforschung Gesundheitsschutz 56, 668-677

Heller T, Blum M, Spraul M, Wolf G, Müller U (2014): Folgeerkrankungen des Diabetes mellitus: Prävalenzen in der Bundesrepublik Deutschland. Dtsch med Wochenschr 139, 786-791

Herold G: Innere Medizin: Eine vorlesungsorientierte Darstellung; unter Berücksichtigung des Gegenstandskataloges für die Ärztliche Prüfung; mit ICD-10 Schlüssel im Text und Stichwortverzeichnis. Herold, Köln 2014

Herxheimer A, McPherson A, Miller R, Shepperd S, Yaphe J, Ziebland S (2000): Database of patients' experiences (DIPEx): a multi-media approach to sharing experiences and information. Lancet $\underline{355}$, 1540-1543

Himmel W, Rönsberg W: Arzt-Patienten-Beziehung in der Allgemeinpraxis; in: Allgemeinmedizin und Familienmedizin: 220 Tabellen; hrsg. v. Abholz H, Kochen M. (Duale Reihe), 4. Auflage; Thieme. Stuttgart 2012, 589-598

Hinder S, Greenhalgh T (2012): "This does my head in". Ethnographic study of self-management by people with diabetes. BMC Health Serv Res 12:83; doi: 10.1186/1472-6963-12-83

Holmström IM, Rosenqvist U (2005): Misunderstandings about illness and treatment among patients with type 2 diabetes. J Adv Nurs $\underline{49}$, 146-154

Honer A: Lebenswelt; in: Hauptbegriffe qualitativer Sozialforschung; hrsg. v. Bohnsack R, Marotzki W, Meuser M; (UTB 8226), 3. Auflage; Budrich, Opladen 2011, 110-112 
Hopper JL (1999): Is type II (non-insulin-dependent) diabetes mellitus not so "genetic" after all? Diabetologia $\underline{42}, 125-127$

Hörnsten A, Sandström H, Lundman B (2004): Personal understandings of illness among people with type 2 diabetes. J Adv Nurs $\underline{47}, 174-182$

Ingadottir B, Halldorsdottir S (2008): To Discipline a "Dog": The Essential Structure of Mastering Diabetes. Qual Health Res $\underline{18}$, 606-619

International Diabetes Federation: IDF Diabetes Atlas, 6. Auflage; International Diabetes Federation, Brüssel 2013. Online unter: http://www.idf.org/diabetesatlas/download-book (aufgerufen am 24.09.2014)

Jerant AF, von Friederichs-Fitzwater MM, Moore M (2005): Patients' perceived barriers to active self-management of chronic conditions. Patient Educ Couns $\underline{57}, 300-307$

Kleinman A: The illness narratives: Suffering, healing \& [and] the human condition; Basic Books, Cambridge 1988

Kneck $\AA$ : Learning to live with diabetes - as experiencing an expanding life world, Karolinska Institutet, Stockholm 2011. Online unter: http://hdl.handle.net/10616/40428 (aufgerufen am 24.09.2014)

Kogan AJ (2009): Overcoming obstacles to effective care of type 2 diabetes. Am J Manag Care $\underline{15}, 255-262$

Koller H: Hermeneutik; in: Hauptbegriffe qualitativer Sozialforschung; hrsg. v. Bohnsack R, Marotzki W, Meuser M; (UTB 8226), 3. Auflage; Budrich, Opladen 2011, 83-85

Köster I, von Ferber L, Ihle P, Schubert I, Hauner H (2006): The cost burden of diabetes mellitus: the evidence from Germany - the CoDiM study. Diabetologia 49, 1498-1504

Kreher S: Hausärztliche Krankheitskonzepte: Analyse ärztlicher Vorstellungen zu Kopfschmerzen, akutem Husten, Ulcus cruris und Schizophrenie. Huber, Bern 2009

Kumagai AK, Murphy EA, Ross PT (2009): Diabetes stories: use of patient narratives of diabetes to teach patient-centered care. Adv Health Sci Educ Theory Pract 14, 315-326

Landgraf R, Kellerer M, Fach E, Gallwitz B, Hamann A, Joost H, Klein H, Müller-Wieland D, Nauck M, Reuter $\mathrm{H}$ et al. (2013): Praxisempfehlungen DDG/DGIM. Diabetologie und Stoffwechsel $\underline{8}, 146-158$

Law S (2011): Using narratives to trigger reflection. Clin Teach $\underline{8}, 147-150$

Lawton J, Peel E, Parry O, Douglas M (2008): Shifting accountability: a longitudinal qualitative study of diabetes causation accounts. Soc Sci Med $\underline{67}, 47-56$

Lawton J, Peel E, Parry O, Araoz G, Douglas M (2005): Lay perceptions of type 2 diabetes in Scotland: bringing health services back in. Soc Sci Med 므, 1423-1435

Legewie H, Schervier-Legewie B (2004): "Forschung ist harte Arbeit, es ist immer ein Stück Leiden damit verbunden. Deshalb muss es auf der anderen Seite Spaß machen": Anselm Strauss im Interview mit Heiner Legewie und Barbara Schervier-Legewie. Forum Qualitative Sozialforschung / Forum: Qualitative Social Research 5(3), Art. 22. Online unter: http://www.qualitative-research.net/index.php/fqs/article/view/562/1218 (aufgerufen am 24.09.2014)

Leventhal H (1971): Fear appeals and persuasion: the differentiation of a motivational construct. Am J Public Health $\underline{61}$, 1208-1224

Lim EL, Hollingsworth KG, Aribisala BS, Chen MJ, Mathers JC, Taylor R (2011): Reversal of type 2 diabetes: normalisation of beta cell function in association with decreased pancreas and liver triacylglycerol. Diabetologia $\underline{54,2506-2514}$ 
Lin EH, von Korff M, Alonso J, Angermeyer MC, Anthony J, Bromet E, Bruffaerts R, Gasquet I, de Girolamo G, Gureje $O$ et al. (2008): Mental disorders among persons with diabetes-results from the World Mental Health Surveys. J Psychosom Res $\underline{65}, 571-580$

Linden M, Nather J, Wilms HU (1988): Zur Definition, Bedeutung und Messung der Krankheitskonzepte von Patienten. Die Krankheitskonzeptskala (KK-Skala) für schizophrene Patienten. Fortschr Neurol Psychiatr $\underline{56}$, 35-43

Lustman PJ, Anderson RJ, Freedland KE, de Groot M, Carney RM, Clouse RE (2000): Depression and poor glycemic control: a meta-analytic review of the literature. Diabetes Care $\underline{23}$, 934-942

Magkos F, Yannakoulia M, Chan JL, Mantzoros CS (2009): Management of the Metabolic Syndrome and Type 2 Diabetes Through Lifestyle Modification. Annu Rev Nutr 29, 223-256

Marx G, Wollny A (2009): Qualitative Sozialforschung - Ausgangspunkte und Ansätze für eine forschende Allgemeinmedizin: Teil 1: Theorie und Grundlagen der qualitativen Forschung. Zeitung für Allgemeinmedizin 85, 467-476

Matthaei S, Bierwirth R, Fritsche A, Gallwitz B, Häring $H$, Joost $H$, Kellerer M, Kloos C, Kunt T, Nauck M et al. (2009): Medical Antihyperglycaemic Treatment of Type 2 Diabetes Mellitus. Exp Clin Endocrinol Diabetes 117, 522-557

Matthaei S, Bierwirth R, Fritsche A, Gallwitz B, Häring $H$, Joost $H$, Kellerer $M$, Kloos $C$, Kunt T, Nauck M et al. (2011): Behandlung des Diabetes mellitus Typ2. Diabetologie und Stoffwechsel $\underline{6}, 131-136$

McNaughton D (2013): 'Diabesity' down under: overweight and obesity as cultural signifiers for type 2 diabetes mellitus. Crit Public Health $\underline{23}$, 274-288

Medici F, Hawa M, lanari A, Pyke DA, Leslie RD (1999): Concordance rate for type II diabetes mellitus in monozygotic twins: actuarial analysis. Diabetologia $\underline{42}, 146-150$

Mendenhall E, Seligman RA, Fernandez A, Jacobs EA (2010): Speaking through Diabetes. Med Anthropol Q 24 , 220-239

Meuser: Interpretatives Paradigma; in: Hauptbegriffe qualitativer Sozialforschung; hrsg. v. Bohnsack R, Marotzki W, Meuser M; (UTB 8226), 3. Auflage; Budrich. Opladen 2011, 92-94

Meyer D, Leventhal H, Gutmann M (1985): Common-sense models of illness: the example of hypertension. Health Psychol 4, 115-135

Miller DP, Spangler JG, Vitolins MZ, Davis SW, Ip E (2013): Are Medical Students Aware of Their Anti-obesity Bias? Acad Med 88, 978-982

Mokdad AH, Bowman BA, Ford ES, Vinicor F, Marks JS, Koplan JP (2001): The continuing epidemics of obesity and diabetes in the United States. JAMA 286, 1195-1200

Moser A, van der Bruggen H, Spreeuwenberg C, Widdershoven G (2008): Autonomy through identification: a qualitative study of the process of identification used by people with type 2 diabetes. J Clin Nurs $\underline{17}$, 209-216

Mühlhauser I, Lenz M (2008): Verbesserung der Therapieergebnisse durch Patientenwissen? Z Evid Fortbild Qual Gesundhwes 102, 223-230

Nasseh K, Frazee SG, Visaria J, Vlahiotis A, Tian Y (2012): Cost of Medication Nonadherence Associated With Diabetes, Hypertension, and Dyslipidemia. Am J Pharm Benefits 4, e41-e47. Online unter: http://www.ajmc.com/publications/ajpb/2012/ajpb_marapr2012/Cost-of-Medication-Nonadherence-Associated-With-Diabetes-Hypertension-and-Dyslipidemia (aufgerufen am 24.09.2014) 
Nield L, Summerbell CD, Hooper L, Whittaker V, Moore H (2008): Dietary advice for the prevention of type 2 diabetes mellitus in adults. Cochrane Database Syst Rev 16, CD005102; doi: 10.1002/14651858.CD005102.pub2

Nolan CJ, Damm P, Prentki M (2011): Type 2 diabetes across generations: from pathophysiology to prevention and management. Lancet $\underline{378}, 169-181$

Parry O, Peel E, Douglas M, Lawton J. (2005): Issues of cause and control in patient accounts of Type 2 diabetes. Health Educ Res $\underline{21}, 97-107$

Parsons T (1975): The sick role and the role of the physician reconsidered. Milbank Mem Fund $Q$ Health Soc $\underline{53}, 257-278$

Paterson B (2001): Myth of empowerment in chronic illness. J Adv Nurs 34, 574-581

Paterson B, Thorne S (2000): Developmental evolution of expertise in diabetes self-management. Clin Nurs Res $\underline{9}, 402-419$

Paterson BL, Russell C, Thorne S (2001): Critical analysis of everyday self-care decision making in chronic illness. J Adv Nurs $\underline{35}$, 335-341

Peel E, Parry O, Douglas M, Lawton J (2004): Blood glucose self-monitoring in non-insulintreated type 2 diabetes: a qualitative study of patients' perspectives. $\mathrm{Br} \mathrm{J}$ Gen Pract $\underline{54}, 183-$ 188

Péres DS, Franco LJ, dos Santos MA (2008): Feelings of women after the diagnosis of type 2 diabetes. Rev Lat Am Enfermagem 16, 101-108

Peyrot M, Rubin RR, Lauritzen T, Snoek FJ, Matthews DR, Skovlund SE (2005): Psychosocial problems and barriers to improved diabetes management: results of the Cross-National Diabetes Attitudes, Wishes and Needs (DAWN) Study. Diabet Med 22, 1379-1385

Pfeffer S: Krankheit und Biographie: Bewältigung von chronischer Krankheit und Lebensorientierung. 1. Auflage; VS Verlag für Sozialwissenschaften, Wiesbaden 2010

Poon M, Tarrant M (2009): Obesity: attitudes of undergraduate student nurses and registered nurses. J Clin Nurs $\underline{18}$, 2355-2365

Poulsen P, Kyvik KO, Vaag A, Beck-Nielsen H (1999): Heritability of type II (non-insulin-dependent) diabetes mellitus and abnormal glucose tolerance--a population-based twin study. Diabetologia $\underline{42}, 139-145$

Poulsen P, Grunnet LG, Pilgaard K, Storgaard H, Alibegovic A, Sonne MP, Carstensen B, Beck-Nielsen H, Vaag A (2009): Increased risk of type 2 diabetes in elderly twins. Diabetes $\underline{58}, 1350-1355$

Puhl RM, Moss-Racusin CA, Schwartz MB (2007): Internalization of weight bias: Implications for binge eating and emotional well-being. Obesity (Silver Spring) 15, 19-23

Räisänen U, Bekkers M, Boddington P, Sarangi S, Clarke A (2006): The causation of disease - The practical and ethical consequences of competing explanations. Med Health Care Philos 9, 293-306

Rathmann W, Scheidt-Nave C, Roden M, Herder C (2013): Type 2 diabetes: prevalence and relevance of genetic and acquired factors for its prediction. Dtsch Arztebl Int 110, 331-337

Redman BK (2007): Responsibility for control; Ethics of patient preparation for self-management of chronic disase. Bioethics 21, 243-250

Rhodes ET, Prosser LA, Hoerger TJ, Lieu T, Ludwig DS, Laffel LM (2012): Estimated morbidity and mortality in adolescents and young adults diagnosed with Type 2 diabetes mellitus. Diabet Med 29, 453-463 
Riemann G: Narratives Interview; in: Hauptbegriffe qualitativer Sozialforschung; hrsg. v. Bohnsack R, Marotzki W, Meuser M. (UTB 8226), 3. Auflage; Budrich, Opladen 2011, 120 122

Rosenthal G: Interpretative Sozialforschung: eine Einführung. 3. Auflage, Juventa-Verlag, Weinheim 2011

Röcke W, Weitbrecht J: Askese und Identität in Spätantike, Mittelalter und Früher Neuzeit. (Transformationen der Antike, Band 14); De Gruyter, Berlin 2010

Rubin RR (2005): Adherence to pharmacologic therapy in patients with type 2 diabetes mellitus. Am J Med 118 (Supplement 5A), 27-34

Schabert J, Browne JL, Mosely K, Speight J (2013): Social Stigma in Diabetes. Patient $\underline{6}$, $1-$ 10

Schneider KL, Andrews C, Hovey KM, Seguin RA, Manini T, Lamonte MJ, Margolis KL, Waring ME, Ning Y et al. (2014): Change in physical activity after a diabetes diagnosis: opportunity for intervention. Med Sci Sports Exerc 46, 84-91

Schorb F: Fit for fun? - Schlankheit als Sozialprestige; in: Risiko Gesundheit: Über Risiken und Nebenwirkungen der Gesundheitsgesellschaft; hrsg. v. Paul B, Schmidt-Semisch H, 1. Auflage; VS Verlag für Sozialwissenschaften, Wiesbaden 2009, 105-121

Schütz A: Gesammelte Aufsätze: Das Problem der sozialen Wirklichkeit (Band 1); Springer Netherlands, Dordrecht 1971

Schwartz MB, Chambliss HO, Brownell KD, Blair SN, Billington C (2003): Weight bias among health professionals specializing in obesity. Obes Res 11, 1033-1039

Schwarzer R, Jerusalem M (2002): Das Konzept der Selbstwirksamkeit. Zeitschrift für Pädagogik Beiheft 44, 28-53

Seidl E, Walter I, Rappold E: Diabetes. Der Umgang mit einer chronischen Krankheit. Böhlau 2007

Shaw J, Sicree R, Zimmet P (2010): Global estimates of the prevalence of diabetes for 2010 and 2030. Diabetes Res Clin Pract 87, 4-14

Stefan N, Kantartzis K, Machann J, Schick F, Thamer C, Rittig K, Balletshofer B, Machicao F, Fritsche A, Häring HU (2008): Identification and characterization of metabolically benign obesity in humans. Arch Intern Med 168, 1609

Steinke I: Kriterien qualitativer Forschung: Ansätze zur Bewertung qualitativ-empirischer Sozialforschung. Juventa, Weinheim 1999

Strauss AL: Grundlagen qualitativer Sozialforschung: Datenanalyse und Theoriebildung in der empirischen soziologischen Forschung (UTB für Wissenschaft 1776), 2. Auflage; Fink, München 1998

Strauss AL, Corbin JM: Grounded theory: Grundlagen qualitativer Sozialforschung. Beltz, PsychologieVerlagsUnion, Weinheim 1996

Strübing J: Theoretisches Sampling; in: Hauptbegriffe qualitativer Sozialforschung; hrsg. v. Bohnsack R, Marotzki W, Meuser M. (UTB 8226), 3. Auflage; Budrich, Opladen 2011, 154156

Teachman BA, Brownell KD (2001): Implicit anti-fat bias among health professionals: is anyone immune? Int J Obes Relat Metab Disord 25, 1525-1531

Teixeira ME, Budd GM (2010): Obesity stigma: A newly recognized barrier to comprehensive and effective type 2 diabetes management. J Am Acad Nurse Pract 22, 527-533 
Terry T, Raravikar K, Chokrungvaranon N, Reaven PD (2012): Does aggressive glycemic control benefit macrovascular and microvascular disease in type 2 diabetes? Insights from ACCORD, ADVANCE, and VADT. Curr Cardiol Rep 14, 79-88

The Action to Control Cardiovascular Risk in Diabetes Study Group (2008): Effects of intensive glucose lowering in type 2 diabetes. N Engl J Med $\underline{358}, 2545-2559$

The ADVANCE collaborative group (2008): Intensive blood glucose control and vascular outcomes in patients with type 2 diabetes. N Engl J Med 358, 2560-2572

Thomas MC, Zimmet P, Shaw JE (2006): Identification of obesity in patients with type 2 diabetes from Australian primary care: the NEFRON-5 study. Diabetes Care 29, 2723-2725

Thorne SE, Ternulf Nyhlin K, Paterson BL (2000): Attitudes toward patient expertise in chronic illness. Int J Nurs Stud 37, 303-311

Toeller M (2005): Evidenz-basierte Ernährungsempfehlungen zur Behandlung und Prävention des Diabetes mellitus. Ernährungsumschau 14, 75-94

Toombs SK: The Meaning of Illness: A Phenomenological Account of the Different Perspectives of Physician and Patient. (Philosophy and Medicine), Springer Netherlands, Dordrecht 1992

Tuomilehto J, Lindström J, Eriksson JG, Valle TT, Hämäläinen H, Ilanne-Parikka P, KeinänenKiukaanniemi S, Laakso M, Louheranta A, Rastas M et al. (2001): Prevention of type 2 diabetes mellitus by changes in lifestyle among subjects with impaired glucose tolerance. $\mathrm{N}$ Engl $\mathrm{J}$ Med 344, 1343-1350

Turner BS: The Body and Society: Explorations in Social Theory (Theory, culture \& society); Sage Publications, London 2008

UK Prospective Diabetes Study (UKPDS) Group (1998a): Intensive blood-glucose control with sulphonylureas or insulin compared with conventional treatment and risk of complications in patients with type 2 diabetes (UKPDS 33). Lancet 352, 837-853

UK Prospective Diabetes Study (UKPDS) Group (1998b): Effect of intensive blood-glucose control with metformin on complications in overweight patients with type 2 diabetes (UKPDS 34). Lancet $\underline{352}, 854-865$

Webb H (2009): 'I've put weight on cos l've bin inactive, cos l've 'ad me knee done': moral work in the obesity clinic. Sociol Health IIIn 31, 854-871

Whittemore R, Chase SK, Mandle CL, Roy C (2002): Lifestyle change in type 2 diabetes a process model. Nurs Res $\underline{51}$, 18-25

World Health Organisation (2013): Diabetes. Fact sheet №312. Online unter: http://www.who.int/mediacentre/factsheets/fs312/en/ (aufgerufen am 25.09.2014)

Yamakawa M, Makimoto K (2008): Positive experiences of type 2 diabetes in Japanese patients: an exploratory qualitative study. Int J Nurs Stud $\underline{45}$, 1032-1041

Yannakoulia M (2006): Eating behavior among type 2 diabetic patients: a poorly recognized aspect in a poorly controlled disease. Rev Diabetic Stud $\underline{3}, 11-16$ 


\section{Anhang}

\subsection{Memo zur Kernkategorie Verzicht}

Die Aufforderung den eigenen Lebensstil zu verändern, wird zu Beginn der Krankheit, manchmal auch während des gesamten weiteren Verlaufs, von vielen Interviewten mit einem Verzicht auf alle schönen Dinge im Leben gleichgesetzt.

Offenbar empfinden viele Interviewten einen hohen Druck, Genuss allgemein zu entsagen - wobei erschwerend hinzuzukommen scheint, dass sie noch nicht einmal von außen dazu gezwungen werden oder „eingesperrt" sind - sondern sich selbst zum Verzichten zwingen müssen. Währenddessen scheinen die Mitmenschen unbeschwert zu „schlemmen" und von überall angebotenen und beworbenen Speisen geht eine quälende Versuchung aus. Seinen Verzicht zu erklären bedeutet, sich von seinem Umfeld abzugrenzen und ein moralisches Prinzip für sich zu erheben, das dann auch konsequent befolgt werden muss. Die Vorstellung davon, auf welche Art und Weise genau verzichtet werden muss, scheint aber beliebig. Trotz der Flut an Ernährungsregeln und Bewegungstipps ist kein „richtiges“ Verzichten festgelegt, welches Orientierung für das eigene Verhalten bietet. Einige deuten eine stark emotional geprägte Wahrnehmung an, dass es gerade ihre individuellen Wünsche sind, die sie sich nicht erfüllen dürfen.

Viele der Interviewten empfinden die Forderung des ärztlichen Personals als genussfeindlich und lebensfern. Sie erklären, dass Ärztinnen und Ärzte keine konkreten Hilfestellungen geben, wie das Verzichten durchzuführen ist. Stattdessen richten diese ihre Aufmerksamkeit auf die vermeintlich objektiven Produkte der persönlichen Bemühungen, nämlich auf die „sichtbaren“ Werte und Körpermaße. Daran wird kritisiert, dass Ärztinnen und Ärzte nur Momentaufnahmen erfassen und unmöglich die Anstrengung abschätzen können, die, mit der Aufgabe zu verzichten, einhergehen. Sie werden dabei zu von der Lebenswelt der Interviewten entfernten moralischen „Richtern“. Die Wahrnehmung der Interviewten, dass großer Druck auf sie ausübt wird, kann zu einer - durch eben diese Wahrnehmung legitimierten - Trotzreaktion führen. Verhandlungen über Verzicht werden mit dem ärztlichen Personal wie persönliche Konflikte ausgetragen. Kränkungen und Enttäuschung über mangelnde Empathie von Ärztinnen und Ärzten, die scheinbar selbst nicht verzichten müssen, führen zu dem Schluss, in einem solchen Konflikt zwangsläufig zu unterliegen. 
Während Verzicht in der ärztlichen Sprechstunde auf einer abstrakten Ebene diskutiert wird, bildet das Alltagsleben den Rahmen für die praktische Auseinandersetzung mit Verzicht. Zur Orientierung werden ganz persönliche „Regeln“ entwickelt, z.B. welche Lebensmittel "gesund“ und welche „ungesund“ sind. Da jede Person allein verzichten muss, hat sie auch den Spielraum zu entscheiden, auf was sie verzichtet und auf welche Weise: ob sie ihre Ernährungsgewohnheiten z.B. radikal oder nur teilweise umstellt. „Regeln“ bieten die Möglichkeit richtig und falsch konkret zu definieren und machen die Person so überhaupt erst handlungsfähig. Dabei kann Verzicht zum einen so gestaltet werden, dass er zu den eigenen Bedürfnissen passt und damit nicht mehr unerträglich erscheint. Zum anderen wird Verzichten dann möglicherweise zu einer selbstständigen Entscheidung, statt eine ärztliche Forderung zu bleiben.

Wichtig scheint dabei nicht einmal so sehr die Erkenntnis zu sein, dass der Verlauf der Krankheit in der eigenen Hand liegt - das kann auch Druck erzeugen - sondern, dass es eigene Gestaltungsmöglichkeiten in der Art des Verzichtens gibt. Es scheint dann nicht mehr so, als ob ein Arzt verlangt, sich von allen schönen Dingen loszusagen, sondern dass der "Lösungsweg" zu einer Verbesserung des Gesundheitszustands (z.B. aber nicht nur erkennbar anhand der Laborwerte) freigestellt ist. Als Beispiel möchte ich P32 nennen, der an den Laborwerten erkennt, dass das Fasten, von dem der Arzt inm abgeraten hat, ein erfolgreicher "Lösungsweg“ ist. Dabei kann auch die sonst häufig kritisierte Bewertung nach den Werten zu etwas Positivem werden.

Schließlich kann der Verzicht auf vorher als unverzichtbar geltende Genussmittel zur überraschenden Entdeckung von neuen sinnlich-genussvollen Dingen kommen. Z.B. entdeckt P20, dass sie sich mittlerweile "ganz dekadent" Zeit für sich alleine "gönnt“. P32 beschreibt, wie er überrascht feststellt, dass es durch den Sport auf einmal etwas Entspannung gibt, neben der „Arbeit und den anderen [Familienmitgliedern]“. 